\title{
Characterizing microseismicity at the Rotokawa and Ngatamariki geothermal fields
}

\author{
By Chet Hopp
}

\author{
A thesis \\ submitted to Victoria University of Wellington \\ in fulfillment of the requirements for the degree of \\ Doctor of Philosophy in Geophysics.
}

Victoria University of Wellington

2019 

"Esta vida es igual que un libro, cada página es un día vivido."

Luis Enrique

"There is no bag limit on happiness."

Ted Nugent 



\section{Abstract}

In this thesis, we construct a four-year (2012-2015) catalog of microearthquakes for the Ngatamariki and Rotokawa geothermal fields in the Taupō Volcanic Zone of New Zealand, and use these data to improve the knowledge of reservoir behavior. These microearthquakes occur frequently, often every few seconds, and therefore provide a tool that we use to assess reservoir properties with dense spatial and temporal resolution as well as to illuminate the underlying processes of seismogenesis. Using a matched-filter detection technique we detect and precisely relocate nearly 9000 events, from which we calculate 982 focal mechanisms.

At Ngatamariki, these results constitute the first detailed analysis of seismicity at a newly-developed resource. It has been commonly assumed that induced shear on fractures increases reservoir permeability by offsetting asperities on either fracture wall, thereby propping the fracture open. During stimulation treatments of two boreholes (NM08 and NM09), borehole permeability experiences logarithmic growth. At NM08, this growth occurs for eight days in the absence of seismicity, while at NM09 only nine microearthquakes are observed during the one-month treatment. This suggests that hydro-shear, the process of inducing seismicity through increased pore pressure at critically-stressed fractures, is not the dominant mechanism of permeability increase at many geothermal wells. Instead, aseismic processes, likely thermal and overpressure induced fracture opening, dominate well stimulation in high-temperature geothermal settings.

At Rotokawa, the earthquake frequency-magnitude distribution ( $b$-value) is positively correlated with both proximity to major injection wells and depth. In an inferred pressure compartment near injection well RK23, $b$ is $\sim 1.18$, but is $<1.0$ elsewhere, suggesting a connection between increased pore-fluid pressure and small-magnitude events. In addition, throughout the reservoir $b$ increases from a value of $\sim 1.0$ at injection depth to almost 1.5 two kilometers below the reservoir, consistent with observations at volcanic areas elsewhere, but opposing the conventional wisdom that $b$-value is inversely proportional to differential stress.

Finally, the 982 focal mechanism observations that we invert for stress show a normal faulting regime throughout both reservoirs. At Rotokawa, a lowering stress ratio, $\nu$, after reintroduction of injection well RK23 ( $\nu$ drops from 0.9 to 0.2 over six months) indicates that anisotropic reservoir cooling affects the reservoir stress state through a process of preferential stress reduction. 


\section{Acknowledgments}

I wish to acknowledge, first and foremost, the patience, guidance and support of my supervisors Martha Savage and John Townend, who allowed me to careen from project to project, eventually happening upon the document you're holding, and learning an immeasurable amount in the process. Thank you also to Steve Sherburn, Sandra Bourguignon, Zara Rawlinson and the rest of the team at GNS Science for installing and maintaining the seismic network used here, for providing advice and scientific context and for creating the preliminary earthquake catalog from which all of this began.

A very large thank you indeed to Mercury NZ Ltd. (the artist formerly known as Mighty River Power), the Tauhara No. 2 Trust and the Rotokawa Joint Venture for fully funding all aspects of this project and for generously providing access to the large and varied dataset from which I learned so much. A similarly large thank you goes out to the fantastic, helpful staff at the New Zealand eScience Infrastructure for providing many (misused) hours of computing time on the PAN cluster and for their patience when things often went awry.

To the staff on floor 5 of the Cotton building including: Rupert, Huw, Tim and Adrian, thank you for providing banter, lunchtime monologues, pertinent skiing advice and unparalleled swagger. To the glue holding this circus together, Aleksandr Beliaev, many thanks for being exceptionally helpful and always willing to share an encyclopedic knowledge of the world of computing.

I would be remiss in forgetting to acknowledge each of the cohort of graduate students to have passed through these halls while I have been here including: Jesse, Rachel, Franny, LMB, Gabe, Stefan, Hamish, Cécile, Abhi, Pasan, Hubert, Katrina, Weiwei, Nico, Dan, Konrad, Lucie and all of the others I may have missed. Thank you for making this place a lively space full of character and fun. To my New Zealand family Dani, Andy, Kostas, Dom, B-Chow, Lauren, Jamey, KJ, Calum, Emily, Geoff, Elisa, Céline and the Hoogs, thank you for making this place home.

Thank you to Stefan for his diligent work in conducting the automated S-picking used throughout this work and for having an appreciation for real hockey.

I want to specifically thank Mr. Calum J. Chamberlain for serving as a de-facto supervisor over the last three-and-a-bit years. Thank you for providing me with a sterling example of what effort, caring and lovely code looks like. 
Without the vision of Steve Sewell, in particular, this project would never have existed. To Steve, I offer the most sincere thank you for opening up his deep knowledge of all things Ngatamariki, Rotokawa and geothermal from which I could always draw when I was confused. This project wouldn't be half as good without your contribution. Beers on me for life.

To Ashley, 'thank you' is woefully inadequate. For patiently stumbling into a threeyear experiment of much greater proportions and difficulty than this $\mathrm{PhD}$, you have my unending appreciation and gratitude.

Finally, to Mom and Dad, your contribution to this accomplishment is immeasurable. Thank you for absolutely everything. This is for you. 


\section{Contents}

\begin{tabular}{ll}
\hline Abstract & i
\end{tabular}

Acknowledgments

List of figures $\quad$ xi

List of tables $\quad$ xiii

\begin{tabular}{|l|l}
\hline Abbreviations and units & xv
\end{tabular}

$\begin{array}{ll}\text { Glossary } & \text { xvii }\end{array}$

\begin{tabular}{lll}
\hline & Introduction & 1
\end{tabular}

1.1 Motivation . . . . . . . . . . . . . . . . . . . . . . . 1

1.2 Geothermal power . . . . . . . . . . . . . . . . . . 2

1.2 .1 Power production . . . . . . . . . . . . . . . . . 2

1.3 Reservoir management . . . . . . . . . . . . . . . . . . . . . . . . . . 3

1.3 .1 Datasets . . . . . . . . . . . . . . . . . . 3

1.3 .2 Terms and definitions . . . . . . . . . . . . . 6

1.4 Geology, development and conceptual models . . . . . . . . . . . . . . . 10

1.4.1 Taupō Volcanic Zone . . . . . . . . . . . . . . . . . . . . . . . . 10

1.4 .2 Ngatamariki . . . . . . . . . . . . . . . . . . . . . . 11

1.4 .3 Rotokawa . . . . . . . . . . . . . . . . . . . . . . . . . 19

1.5 Induced seismicity $\ldots \ldots \ldots \ldots \ldots$. . . . . . . . . . . . . . 26

1.5.1 Settings of injection-induced seismicity . . . . . . . . . . . 27

1.5 .2 Mechanisms of induced seismicity . . . . . . . . . . . . . . . . . 29

1.5 .3 Permeability and seismicity . . . . . . . . . . . . . . 33

1.6 Study aims . . . . . . . . . . . . . . . . . . . . . . . . . . . . . . . 36

1.7 Thesis structure . . . . . . . . . . . . . . . . . . . . . . . . . 37

$2 \quad$ Data and methods 39

2.1 Data $\ldots \ldots \ldots \ldots \ldots \ldots$

$2.1 .1 \quad$ Seismic network . . . . . . . . . . . . . . . . . . . . . . . 39

2.1 .2 Preliminary earthquake catalog . . . . . . . . . . . . . . 40

2.1 .3 Mercury datasets . . . . . . . . . . . . . . . . . . 45 
2.2 Methods . . . . . . . . . . . . . . . . . . . . . . . . . . . . . . . 48

$2.2 .1 \quad$ Earthquake detection $\ldots \ldots \ldots \ldots \ldots$

2.2 .2 Arrival time picking for matched-filter detections $\ldots \ldots \ldots . . \quad 52$

2.2 .3 Earthquake location $\ldots \ldots \ldots \ldots \ldots$

2.2 .4 Magnitude calculation $\ldots \ldots \ldots \ldots \ldots$

2.2 .5 Focal mechanisms . . . . . . . . . . . . . . . . . . . . 65

2.2 .6 Stress inversion $\ldots \ldots \ldots \ldots \ldots$

3 Seismic response to injection well stimulation in a high-temperature, $\begin{array}{ll}\text { high-permeability reservoir } & 71\end{array}$

3.1 Introduction . . . . . . . . . . . . . . . . . . . . . . . . 72

3.1 .1 Geological and geophysical setting . . . . . . . . . . . . . 74

3.1.2 Mechanisms of microseismicity and permeability enhancement . 74

$3.1 .3 \quad$ Ngatamariki power plant operations . . . . . . . . . . . 77

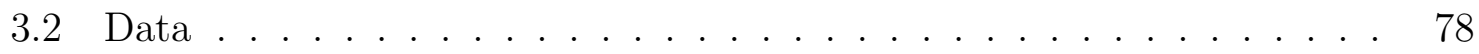

3.3 Methods . . . . . . . . . . . . . . . . . . . . . . . . . . . . 79

3.3 .1 Matched-filter detection . . . . . . . . . . . . . . . . 79

3.3 .2 Detection location $\ldots \ldots \ldots \ldots \ldots$

3.3 .3 Magnitudes . . . . . . . . . . . . . . . . . . . . . . . . . . 81

3.3 .4 Focal mechanisms and stress inversion . . . . . . . . . . . . 82

3.4 Results . . . . . . . . . . . . . . . . . . . . . . . . . . . . . . . . . . . 83

3.4 .1 Matched-filter detection . . . . . . . . . . . . . . 83

3.4 .2 Event location . . . . . . . . . . . . . . . . . . . . . . . 83

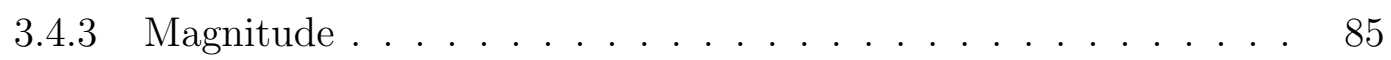

3.5 Discussion $\ldots \ldots \ldots \ldots \ldots$

3.5 .1 Northern injection zone. . . . . . . . . . . . . . . . . . 88

3.5 .2 Southern injection zone $\ldots \ldots \ldots$. . . . . . . . . . . 96

3.5 .3 Focal mechanisms and stress . . . . . . . . . . . . . . . . 100

3.6 Conclusions $\ldots \ldots \ldots \ldots \ldots$

3.7 Acknowledgments . . . . . . . . . . . . . . . . . . . . . . 106

3.8 Appendices $\ldots \ldots \ldots \ldots \ldots$

3.8 .1 Template locations $\ldots \ldots \ldots \ldots$

$3.8 .2 \quad$ NM09 stimulation and testing . . . . . . . . . . . . . 106

3.8 .3 Seismicity-based reservoir characterization . . . . . . . . . . 108

3.8 .4 Maximum magnitude . . . . . . . . . . . . . . . . . . . . . 109

4 Seismic response to evolving injection at the Rotokawa geothermal $\begin{array}{ll}\text { field, New Zealand } & 113\end{array}$

4.1 Introduction . . . . . . . . . . . . . . . . . . . . . . . . . . 113

$4.1 .1 \quad$ Rotokawa resource development . . . . . . . . . . . . . . . 115 
4.1 .2 Rotokawa operations changes . . . . . . . . . . . . . . . 115

4.1 .3 Reservoir model . . . . . . . . . . . . . . . . . . . . . . . . . . . . 117

4.1 .4 Previous work . . . . . . . . . . . . . . . . . . . . . . . . . . . . . 119

4.2 Data and methods . . . . . . . . . . . . . . . . . . . . . . . . . . . . 119

4.2 .1 Data $\ldots \ldots \ldots \ldots \ldots$

4.2 .2 Matched-filter detection . . . . . . . . . . . . . . . . . 120

$4.2 .3 \quad$ NonLinLoc locations . . . . . . . . . . . . . . . . . . . . . . . . 120

4.2 .4 Magnitudes . . . . . . . . . . . . . . . . . . . . . . . 120

$4.2 .5 \quad$ GrowClust locations . . . . . . . . . . . . . . . . . . . . . . 121

$4.2 .6 \quad b$-value calculation $\ldots \ldots \ldots \ldots$

4.3 Results . . . . . . . . . . . . . . . . . . . . . . . . . . . . . . . . . . . . 123

4.3 .1 Locations . . . . . . . . . . . . . . . . . . . . . 123

4.3 .2 Magnitudes . . . . . . . . . . . . . . . . 126

$4.3 .3 \quad b$-value mapping . . . . . . . . . . . . . . . . . . . . . . 126

4.4 Discussion $\ldots \ldots \ldots \ldots \ldots$

4.4 .1 Compartmentalization . . . . . . . . . . . . . . . . 128

4.4 .2 Compartment characteristics . . . . . . . . . . . . . . . 130

4.5 Temporal variations in seismicity and injection parameters . . . . . . . 132

$4.5 .1 \quad$ RK24 injectivity decline . . . . . . . . . . . . . . . . 132

$4.5 .2 \quad$ RK23 halt and restart . . . . . . . . . . . . . . . . . 135

4.5.3 Aseismic injection: RK34 drilling and RK21/RK22 . . . . . . . 137

4.6 Spatial $b$-value variations $\ldots \ldots \ldots \ldots$. . . . . . . . . . . . . . 137

4.7 Conclusions $\ldots \ldots \ldots \ldots$

4.8 Appendices $\ldots \ldots \ldots \ldots$. . . . . . . . . . . . . . . . . . . . . . 140

$4.8 .1 \quad b$-value mapping parameters . . . . . . . . . . . . . . . . . . 140

4.8 .2 'Swarm' activity . . . . . . . . . . . . . . . . . . . . . . . 142

$4.8 .3 \quad$ Location sensitivity to S-picks . . . . . . . . . . . . . . . . . . 142

5 Focal mechanisms and stress state at Rotokawa and Ngatamariki 147

5.1 Introduction . . . . . . . . . . . . . . . . . . . . . . . . . . 147

$5.1 .1 \quad$ Stress changes at geothermal reservoirs . . . . . . . . . . . . . 148

$5.1 .2 \quad$ Fractures and stress state at Rotokawa and Ngatamariki . . . . 150

5.2 Data and methods . . . . . . . . . . . . . . . . . . 151

$5.2 .1 \quad$ Earthquake catalog . . . . . . . . . . . . . . . . . 151

5.2 .2 Focal mechanism determination . . . . . . . . . . . . . . . 151

5.2 .3 kmeans clustering . . . . . . . . . . . . . . . . . . . . . . 152

5.2 .4 Stress inversion . . . . . . . . . . . . . . . . . . . . . 152

5.3 Results . . . . . . . . . . . . . . . . . . . . . . . . . . . . . . 155

5.3 .1 Stress inversions . . . . . . . . . . . . . . . . . . 155

5.4 Discussion . . . . . . . . . . . . . . . . . . . . . . . 159 
$5.4 .1 \quad$ Ngatamariki . . . . . . . . . . . . . . . . . . . . . . . . . . . . . . 159

5.4 .2 Rotokawa . . . . . . . . . . . . . . . . . . . . . . 164

5.5 Conclusions $\ldots \ldots \ldots \ldots \ldots \ldots \ldots \ldots$

5.6 Appendices $\ldots \ldots \ldots \ldots \ldots \ldots$

$5.6 .1 \quad$ Focal mechanism and stress inversion parameter sensitivity . . . 170

$5.6 .2 \quad$ AFIT and FMI $\operatorname{logs} \ldots \ldots \ldots \ldots \ldots$

5.6 .3 Borehole vs focal mechanism fracture orientation . . . . . . . 173

$\begin{array}{lll}6 & \text { Synthesis } & 179\end{array}$

6.1 Summary . . . . . . . . . . . . . . . . . . . . . . . . 179

6.1 .1 Ngatamariki . . . . . . . . . . . . . . . . . . . . . . 181

6.1 .2 Rotokawa . . . . . . . . . . . . . . . . . . . . . . . . 183

6.2 Prospects for further research $\ldots \ldots \ldots \ldots$

6.2 .1 Mechanisms of matched-filter detected events . . . . . . . . . 184

6.2 .2 Thermo-hydro-mechanical modeling . . . . . . . . . . . . 185

6.3 Concluding statement $\ldots \ldots \ldots \ldots \ldots$

\begin{tabular}{ll}
\hline Appendices & 189
\end{tabular}

\begin{tabular}{|ll|}
\hline A Alternative detection techniques & 191
\end{tabular}

A.1 Subspace Detection . . . . . . . . . . . . . . . . . . . . . . . . . . . . 191

A.1.1 Subspace detector design . . . . . . . . . . . . . . . . . . 191

A.1.2 Detection statistic . . . . . . . . . . . . . . . . . . . . . 192

A.1.3 Determining a sufficient dimension . . . . . . . . . . . . 192

A.2 EQcorrscan development . . . . . . . . . . . . . . . . . . . . 193 


\section{List of figures}

$1.1 \quad$ Schematic diagram of the Ngatamariki geothermal power plant design . 4

1.2 Schematic diagram of the Rotokawa geothermal power plant designs . . 5

1.3 Injectivity curve for NM08 stimulation . . . . . . . . . . . . . . . . 9

1.4 Location of the geothermal fields in the Taupō Volcanic Zone . . . . . . 12

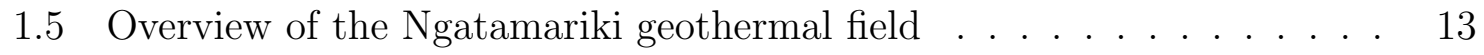

$1.6 \quad$ Detailed cross-section of Ngatamariki geology . . . . . . . . . . . . . . 14

\begin{tabular}{|ll|l|l|l}
1.7 & Flow rates and & wellhead pressures & at Ngatamariki, 2012-2015 . . . . . 17
\end{tabular}

1.8 Cross-section of the Ngatamariki conceptual model . . . . . . . . . . . 18

1.9 Overview of the Rotokawa geothermal field . . . . . . . . . . . . . . . . 21

1.10 Cross-sections of Rotokawa geology . . . . . . . . . . . . . . . . . 22

1.11 Rotokawa flow rates and wellhead pressures, 2012-2015 . . . . . . . . . 24

1.12 Selected cross-sections of the Rotokawa conceptual model . . . . . . . . 25

1.13 Mohr's circles showing the effect of pore-fluid pressure . . . . . . . . . . 31

1.14 Example 'r-t' plot modified from Shapiro et al. [2002)] . . . . . . . . . . . 32

1.15 Mohr's circles showing the effect of thermoelastic stress reduction . . . 34

1.16 Cartoon of fracture permeability increase . . . . . . . . . . . . . 36

2.1 Seismograph station map . . . . . . . . . . . . . . . . . . . . . 41

2.2 Data continuity . . . . . . . . . . . . . . . . . . . . . . . . . . . . . 43

2.3 Mercury and GeoNet magnitude relationships . . . . . . . . . . . . . . 44

2.4 GNS template event locations . . . . . . . . . . . . . . . . 45

2.5 Representative pressure-temperature spinner (PTS) datasets . . . . . . 47

2.6 Wellbore image data $\ldots \ldots \ldots \ldots$. . . . . . . . . . . . . . . . . . . . . . . . . . . 48

2.7 Matched-filter-detected waveforms . . . . . . . . . . . . . . . . . . . 50

2.8 Close-up of matched-filter detections . . . . . . . . . . . . . . 51

2.9 High and low signal-to-noise ratio detections $\ldots \ldots \ldots$. . . . . . . . 53

2.10 Example correlation-based pick correction . . . . . . . . . . . . 54

2.11 Example detection alignment . . . . . . . . . . . . . . . 55

2.12 spickerC example picks . . . . . . . . . . . . . . . . . . . 56

2.13 Relative amplitude calculation . . . . . . . . . . . . . . . . . . . 63

$2.14 \mathrm{M}_{c}$ calculation . . . . . . . . . . . . . . . . . 65

2.15 Polarity consistency check . . . . . . . . . . . . . . . . 67

2.16 Focal mechanisms calculation . . . . . . . . . . . . . . . . 68 
2.17 Stress inversion example results $\ldots \ldots \ldots \ldots$. . . . . . . 70

$3.1 \quad$ Overview of the Ngatamariki geothermal field $\ldots \ldots \ldots \ldots$

3.2 Matched-filter-detected waveforms at two signal-to-noise ratios . . . . . 84

3.3 Ngatamariki seismic catalog double-difference locations $\ldots \ldots \ldots$. . . . 86

3.4 Catalog frequency-magnitude distributions $\ldots \ldots \ldots \ldots$. . . . . 87

3.5 Seismicity and injection parameters during NM08 stimulation .... . 90

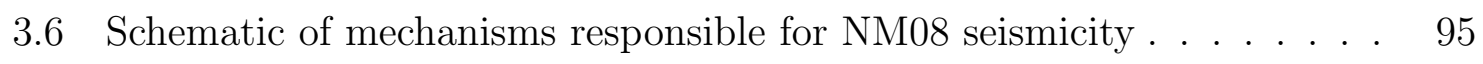

3.7 Focal mechanisms and stress inversions at NM08 and NM10 . . . . . 97

3.8 Seismicity and injection parameters for NM10 drilling . . . . . . . . . 99

3.9 Seismicity and injection parameters for NM10 stimulation $\ldots . . . .101$

3.10 Schematic Mohr's circles for NM08 stimulation . . . . . . . . . . . . . 104

3.11 Template event locations . . . . . . . . . . . . . . . . . . . . 107

3.12 NM09 stimulation parameters $\ldots \ldots \ldots \ldots$

3.13 Diffusion r-t plots for NM08 stimulation $\ldots \ldots \ldots$

3.14 Location uncertainties for NM08 stimulation . . . . . . . . . . . . . 111

3.15 Maximum magnitude with cumulative injected volume . . . . . . . . . 112

4.1 Overview of the Rotokawa geothermal field . . . . . . . . . . 116

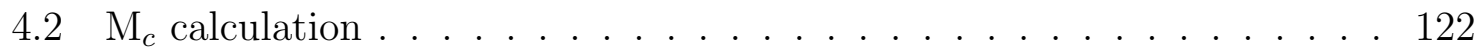

4.3 Final Rotokawa seismic catalog . . . . . . . . . . . . . . . . . . 124

4.4 GrowClust and TomoDD relocation comparison . . . . . . . . . 125

4.5 Catalog frequency-magnitude distributions $\ldots \ldots \ldots \ldots \ldots$

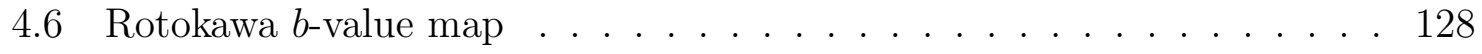

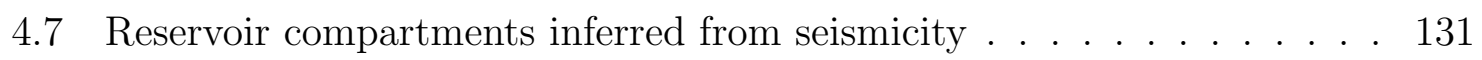

4.8 Characteristics of seismicity in each compartment . . . . . . . . . . 133

4.9 Western compartment seismicity and RK24 injection parameters . . . . 134

4.10 Eastern compartments' seismicity and RK23 injection parameters . . . 136

4.11 Rotokawa $b$-value profiles with depth and event-well distance . . . . . 138

4.12 Additional $b$-value mapping results $\ldots \ldots \ldots \ldots \ldots \ldots$

4.13 Rotokawa 'swarm' dates . . . . . . . . . . . . . . . . . . . . . 143

4.14 Rotokawa 'swarm' locations . . . . . . . . . . . . . . . . . . . 144

4.15 'Swarm' frequency-magnitude distribution . . . . . . . . . . . . 144

4.16 Rotokawa locations: P-picks only . . . . . . . . . . . . . . . 145

4.17 Inferred reservoir compartments: P-picks only . . . . . . . . . . 146

5.1 Overview of the Ngatamariki and Rotokawa geothermal fields . . . . 149

5.2 Ngatamariki focal mechanism solutions $\ldots \ldots \ldots \ldots \ldots$

5.3 Rotokawa focal mechanism solutions $\ldots \ldots \ldots \ldots \ldots$

5.4 Kaverina diagrams of focal mechanism solutions at both fields . . . . 155

5.5 Ngatamariki kmeans clusters . . . . . . . . . . . . . . . . . 157 
$5.6 \quad$ Ngatamariki stress inversions from kmeans clusters . . . . . . . . . . . 158

5.7 Rotokawa kmeans clusters . . . . . . . . . . . . . . . . . . . . . . . 160

5.8 Rotokawa stress inversions from kmeans clusters . . . . . . . . . . . . . 161

5.9 Ngatamariki Clusters 2 and 6 relative to injection flow rate . . . . . . . 163

$5.10 \mathrm{~S}_{\text {Hmax }}$ and $\nu$ for kmeans clusters at Rotokawa . . . . . . . . . . . 166

$5.11 \mathrm{~S}_{\text {Hmax }}$ and $\nu$ with time near injection wells at Rotokawa . . . . . . . . 167

5.12 Effect of thermoelastic cooling on the reservoir stress state . . . . . . . 169

5.13 Focal mechanism uncertainties . . . . . . . . . . . . . . . . . . 171

$5.14 \mathrm{~S}_{\text {Hmax }}$ and $\nu$ with varying clustering parameters . . . . . . . . . . . 172

5.15 Ngatamariki fracture orientations and focal mechanism nodal planes . . 175

5.16 Rotokawa fracture orientations and focal mechanism nodal planes . . . 176

6.1 The combined Ngatamariki-Rotokawa seismic catalog . . . . . . . . . . 182

6.2 Polarity clustering output of Shelly et al. $[(2016 \mathrm{~b})]$. . . . . . . . . . . . . 185

6.3 Example Finite Element Heat and Mass Transfer Code FEHM temperature output . . . . . . . . . . . . . . . . . . . . 187

A.1 Energy capture of subspace detectors with increasing dimension . . . . 193 


\section{List of tables}

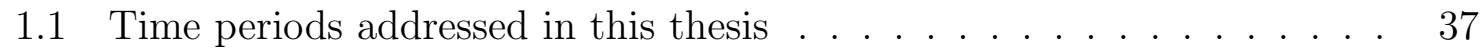

2.1 Seismograph station information . . . . . . . . . . . . . . . . . . 42

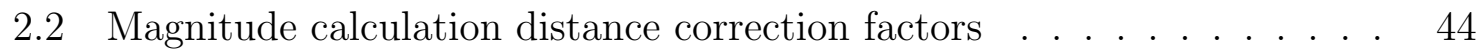

2.3 1-D velocity model used in this thesis . . . . . . . . . . . . . . . . 58

3.1 Summary of pre-startup injection operations $\ldots \ldots \ldots$. . . . . . . . . 78

4.1 Rotokawa injection periods of interest . . . . . . . . . . . . . . . 117 


\section{Abbreviations and units}

II Injectivity Index.

CFF Central Field Fault.

DHP Downhole Pressure.

IFF Injection Field Fault.

MPa Megapascal.

MWe Megawatts electrical.

NAP Nga Awa Purua.

pdf probability density function.

PFF Production Field Fault.

PTS pressure-temperature spinner.

RGEN Rotokawa A.

TVZ Taupō Volcanic Zone.

WHP Wellhead Pressure. 


\section{Glossary}

diffusivity A quantity describing the rate at which fluid particles (or energy) spread or diffuse. Reported as area per unit time $\left(\mathrm{m}^{2} / \mathrm{s}\right)$.

downhole pressure Pressure in a well as measured at a given depth by gas-filled tubing or an installed sensor. Often reported in bar, but here converted to megapascal (MPa) as is the standard for seismology.

feedzone A location of significant permeability in a well. The location(s) into which fluid is inferred to enter or exit the reservoir.

flow rate The volume of fluid being injected into, or extracted from, a well per unit time. Typically reported in New Zealand as tonnes per hour (t/h).

injectivity Shorthand for injectivity index (II; below).

injectivity index A measure of a well's ability to accept a volume of fluid per unit pressure, here reported as $\mathrm{t} / \mathrm{h} / \mathrm{MPa}$.

permeability The ability of a material to transmit fluid.

stimulation A prescribed treatment of a borehole intended to increase its permeability so that more fluid can be injected or extracted.

tracer test Procedure in which a radioactive chemical compound, with known halflife, is injected into a reservoir. Production wells are monitored for the arrival of this compound, the arrival time and concentration of which can be used to infer flow properties of the reservoir..

transmissivity The permeability-thickness product, typically calculated from pressure transient tests.

wellhead pressure Pressure in a well as measured at the wellhead, (approximately the earth's surface). Often reported in bar, but here converted to megapascal $(\mathrm{MPa})$ as is the standard for seismology. 


\section{Introduction}

\subsection{Motivation}

The heat present in the earth's crust has been recognized as a potential energy source for electricity generation for nearly a century, and has been exploited for nearly 60 years in multiple locations (Gregg, 1958; Garrison, 1972; Minissale, 1991). To date, the worldwide installed geothermal capacity is over $14 \mathrm{GW}$ spanning more than 50 countries (Pan et al., 2019, Bertani, 2016), with the potential for substantially more through the creation of enhanced geothermal systems (EGS; Blackwell et al., 2007; Mock et al., 1997). Due to the confluence of abundant, naturally-fractured reservoirs, high heat flow and relatively low startup cost, New Zealand was one of the first countries to adopt geothermal as a source of electricity and currently has the fifth largest installed geothermal capacity in the world, accounting for roughly 16 percent of the nation's electricity generation (Bertani, 2016, Grant and Bixley, 2011). However, the future growth of geothermal technologies is uncertain due to a number of associated risks including high startup costs (Olasolo et al. 2016) and the potential impact of induced seismicity related to injection and withdrawal of fluids Majer et al., 2007; Ellsworth, 2013; Ge et al., 2019).

In the last decade, interest in induced seismicity has grown in the scientific and industrial communities and public sphere (Ellsworth, 2013). This is largely a result of the remarkable increase in seismicity in Oklahoma, Arkansas, Texas and Ohio, in particular, where wastewater from hydrocarbon production is injected into the subsurface (e.g. Yeck et al., 2017; Horton, 2012; Kim, 2013; Mc Garr and Barbour, 2017; Keranen et al. . 2013). In addition, a number of geothermal projects have been abandoned or postponed due to the occurrence of larger-than-expected seismic events (e.g. Deichmann and Giardini, 2009, Kim et al., 2018; Grigoli et al., 2018; Ge et al., 2019). Given the incomplete understanding of the processes that generate such seismicity, it is difficult to reliably forecast the extent, magnitude and timing of seismicity for a given injection or extraction scenario. However, important factors likely include injection parameters such as pressure, flow rate, temperature, total volume and proximity to the basement formation, as well as reservoir properties like temperature, permeability, volume, structure and in situ stress state (Segall and Fitzgerald, 1998; Bachmann et al., 2012; Ellsworth, 2013; McGarr, 2014; Jeanne et al., 2015a; Goebel and Brodsky, 2018). As public and regulatory scrutiny of induced seismicity increases, it will 
become increasingly important to understand the detailed interactions between these factors in order to design strategies to mitigate the risk of generating large, damaging earthquakes (e.g. Langenbruch and Zoback, 2017).

When viewed from a different perspective, the occurrence of human-induced seismicity presents an opportunity to observe earthquakes in locations and at times that that can be accurately forecast a priori. The ability to generate seismicity through controlled, measurable actions offers researchers the ability to study, in great detail, the processes that lead to seismogenesis (e.g. Raleigh et al., 1976). These results can then be applied more widely to problems of natural (and often more damaging) seismicity.

Induced seismicity also provides useful information regarding reservoir properties such as flow pathways, permeability distribution and fracture geometry. From an operational standpoint, this information is critical in managing a dynamic resource like a geothermal reservoir (Majer et al., 2007). This final point is the key to the work presented in this thesis.

In this work, we focus on the Ngatamariki and Rotokawa geothermal fields in the Taupō Volcanic Zone (TVZ) on the North Island of New Zealand, from mid-2012 until the end of 2015. Both fields are operated by Mercury NZ, Ltd. The catalog of seismicity we have created is an indicator of processes occurring within the reservoirs that are otherwise difficult to analyze without drilling additional wells (and spending significantly more money). These catalogs offer clues to the orientation and size of the fracture network, geometry of flow pathways, reservoir transmissivity, and stress state in the reservoir. This insight is especially useful where reservoir behavior cannot be fully explained by borehole-based measurements.

\subsection{Geothermal power}

\subsubsection{Power production}

Geothermal electricity generation relies on converting the heat energy from the earth to mechanical energy, which is used to turn a generator and thus produce electrical energy. Heat is extracted from the subsurface through extraction of a conductivelyheated gas or liquid phase from the reservoir rock. Therefore, a commercially viable geothermal resource requires high temperature (at drillable depths) and sufficient permeability, in order to enable rapid transport of fluid to the wellbore. As a general rule, the temperature of the earth's crust increases with depth at an average of $30^{\circ} \mathrm{C} / \mathrm{km}$ (Grant and Bixley, 2011; DiPippo, 2016). On a more localized level, however, gradients of $100^{\circ} \mathrm{C} / \mathrm{km}$ or greater can be encountered (Grant and Bixley, 2011; DiPippo, 2016). All modern, produced geothermal fields are convective systems, meaning that the pressure and temperature distribution in the field is governed not by conductive 
heat transfer, but by the circulation of hot fluids through permeable rock Grant and Bixley, 2011; DiPippo, 2016). These types of fields can be broken into two basic types; liquid-dominated and, less commonly, vapor-dominated, based on the dominant fluid phase present in the reservoir. Both Ngatamariki and Rotokawa are liquid-dominated reservoirs (McNamara et al., 2016; Chambefort et al., 2016).

There are a variety of field configurations, but most modern geothermal fields consist of a production field, where fluid is drawn out of the reservoir, and an injection field where some portion (up to nearly 100 percent) of the extracted fluid is reinjected back into the reservoir. This reinjection is done for a number of reasons, the most important of which is to maintain reservoir pressure and volume in order to avoid degrading the resource (Grant and Bixley, 2011). The injection and production fields are connected via the power plant (or multiple plants) in which the extracted fluid is used either directly or indirectly (i.e. a binary plant) to turn a turbine and generate electricity. There are many variations of the heat-to-electricity conversion system, and the choice of which to use at a particular field is governed by the dominant fluid phase in the reservoir and the reservoir temperature (DiPippo, 2016). For the purposes of this thesis, we are concerned with binary (Figure 1.1), flash-steam (Figure 1.2 a) and combined-cycle (Figure $1.2 \mathrm{~b}$ ) power plants, which are the types of plants installed at Ngatamariki and Rotokawa. A binary plant uses the fluid extracted from the reservoir to boil a highlyvolatile, secondary fluid (such as isopentane) with a much lower boiling point than the reservoir fluid, which is then used to turn a turbine. A flash steam plant, on the other hand, flashes the extracted reservoir fluid to steam, which is directly deployed in the turbine (DiPippo, 2016). Combined cycle plants use both technologies simultaneously.

Two power plants are installed at Rotokawa. The first, a 24 Megawatts electrical MWe combined-cycle plant named Rotokawa A RGEN), was installed in 1997, while the second, a 140 MWe, triple-flash plant named Nga Awa Purua (NAP), was installed in 2010 McNamara et al., 2016). Ngatamariki is the most recently-developed field in New Zealand, with the 82 MWe binary power plant coming online in 2013.

\subsection{Reservoir management}

\subsubsection{Datasets}

Reservoir engineers, modelers, geologists and geophysicists use a number of data streams to assess the state of a given geothermal reservoir. Most of these data are recorded at boreholes drilled into the resource, and provide a wealth of important information DiPippo, 2016; Grant and Bixley, 2011). For instance, during drilling, rock cuttings and core intervals are extracted from the borehole and used to characterize the geology of the reservoir with depth. A number of instruments are then lowered down the 


\section{Ngatamariki}

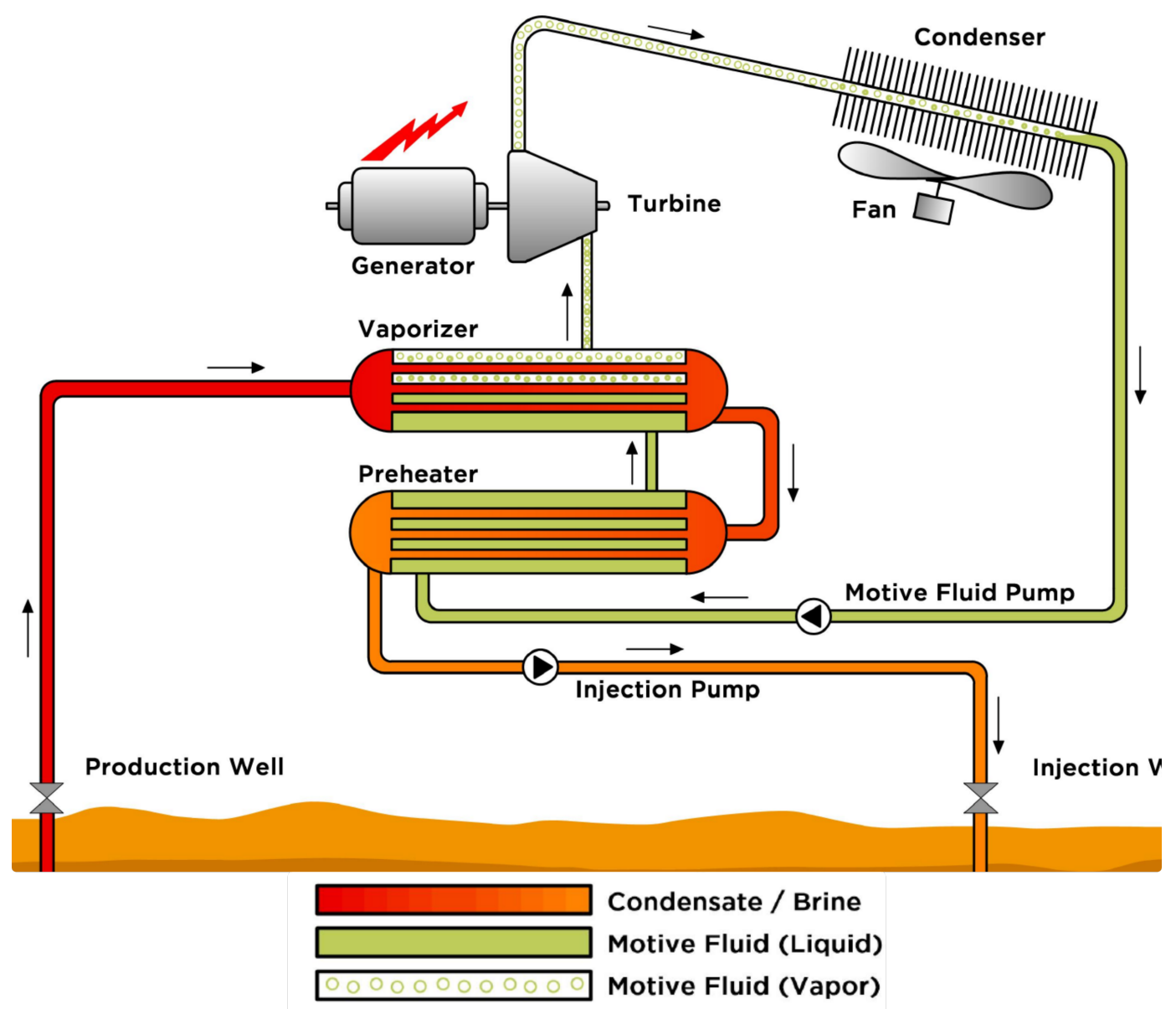

Figure 1.1: Schematic of the operation of a binary power plant, similar to the plant installed at Ngatamariki. This figure is adapted from the ORMAT 2017 Annual Report (Ormat Technologies Inc. 2018).

borehole, measuring temperature, resistivity, porosity, density and fracture spacing/ orientation/aperture. Often, an operator will conduct injection testing of a newlydrilled well. Among other procedures undertaken during an injection test, an impeller/thermometer will be lowered to measure temperature and flow rate with depth (a so-called pressure-temperature spinner (PTS) run), allowing the operator to infer the depth intervals at which injectate exits the well (i.e. feedzones).

During well testing and throughout power plant operation, fluid properties including flow rate, pressure, temperature and chemistry are measured. Also, intermittently, radioactive tracer tests may be conducted. These involve the injection of a chemical compound containing a known quantity of a radioactive isotope, the presence of which is then measured at production wells, providing information about fluid flow rates and paths in the reservoir. 


\section{Rotokawa}
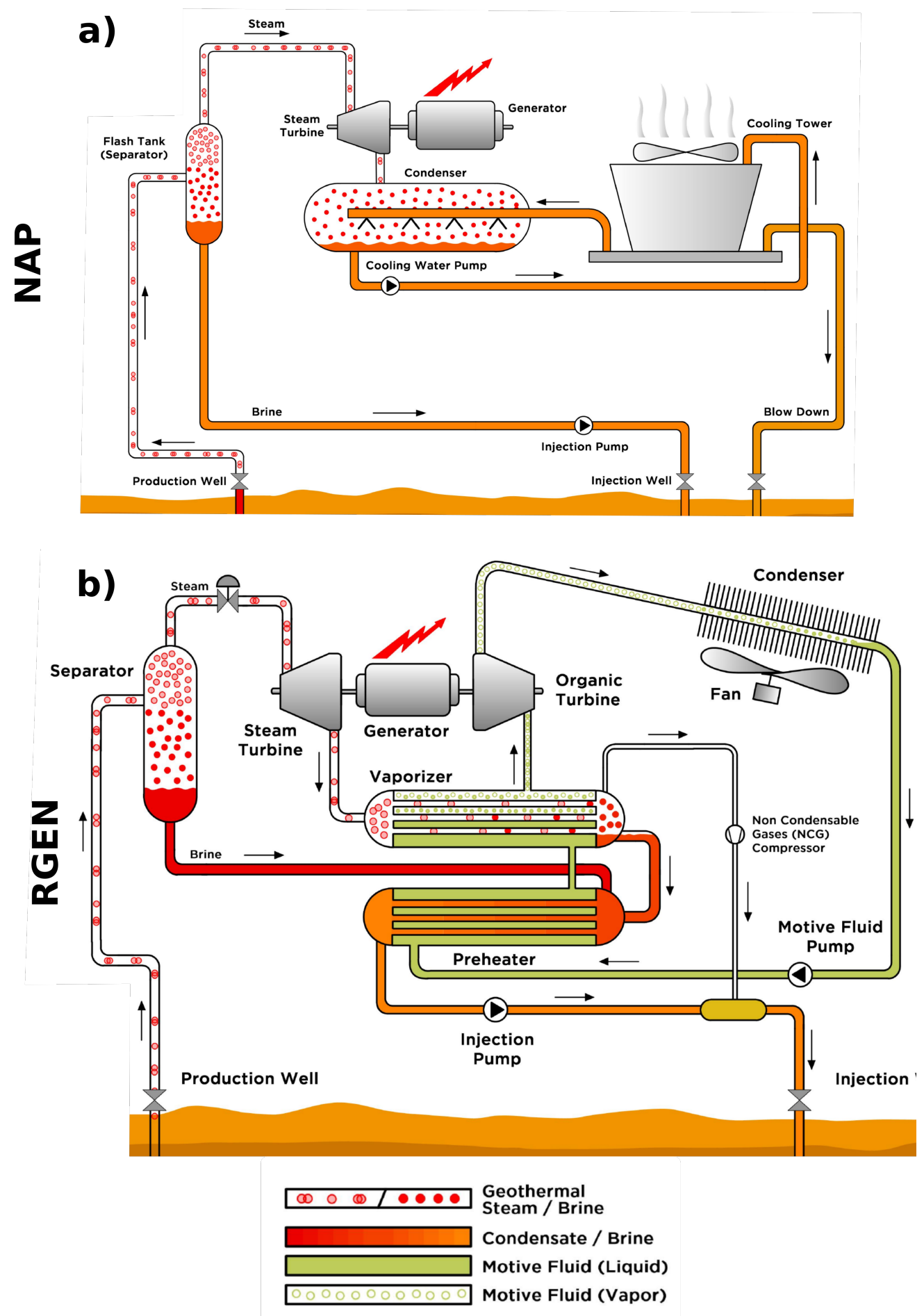

Figure 1.2: Schematic diagrams showing the operation of the two types of geothermal power plants in operation at Rotokawa: a) Flash-steam and b) Combined cycle. At Rotokawa, the original Rotokawa A (RGEN) is type b), while the more recentlyinstalled Nga Awa Purua (NAP) plant is a 'triple-flash' variant of type a), meaning that there are three separate stages of liquid-steam separation, which increases plant efficiency (DiPippo, 2016). These figures are adapted from the ORMAT 2017 Annual Report (Ormat Technologies Inc. 2018) 
These borehole-measured datasets allow operators to constrain the spatial extent, temperature distribution and permeability in the reservoir. However, they are sparselysampled relative to the total reservoir volume due to the prohibitive cost of drilling deep wells. To aid in interpreting the data mentioned above, other geophysical and geochemical data are collected at the surface. Examples of such data include manetotelluric, microgravity and leveling surveys as well as spring/fumarole temperature and chemistry and active- and passive-source seismic waveforms.

Passive-source seismic data occupy a unique place in the spectrum of data available to geothermal operators. While waveform data are collected at the surface (or in some cases at 100s of meters depth in boreholes), they are the surface manifestation of waves emitted by fracture rupture in the reservoir, a process that is affected by the distribution of reservoir temperature and pressure. Pressure and temperature can be accurately measured at boreholes, but seismicity can occur throughout a reservoir and it therefore gives a much more densely-sampled view of fluid movement at depth. For the purposes of this thesis, we will focus on the characterization of reservoir seismicity and its relation to many of the well parameters listed above. The most important constraints on the state of the reservoir come from borehole pressure and flow rate data, both of which will feature prominently in the work that follows.

\subsubsection{Terms and definitions}

When assessing the relationship between seismicity and reservoir management, we generally focus on injection, as opposed to production. This is mostly because the withdrawal of fluids decreases pore-fluid pressure and, therefore, usually stabilizes reservoir fracture networks (Segall et al., 1994; Segall and Fitzgerald, 1998, see further discussion in Section 1.5.2). However, not all injection operations are the same, and each has a specific set of goals that will be important to understand in the context of induced seismicity at Rotokawa and Ngatamariki:

\section{- Reinjection}

During the normal operation of a power plant, brine is extracted from the reservoir, put through the power plant, and reinjected. This type of operation makes up the bulk of the reinjection operations here. In the case of the two fields considered, brine reinjection is characterized by high flow rates (up to $\sim 1000 \mathrm{t} / \mathrm{h}$ ), high temperatures $\left(>90^{\circ} \mathrm{C}\right)$ and injectate chemistry consistent with that of the reservoir fluid. Flash plants also produce a significant amount of condensate, which is the liquid product of the cooling of steam. Most geothermal plants produce this in some form and it must also be reinjected into the reservoir, albeit with a lower temperature (ambient to $\sim 40^{\circ} \mathrm{C}$ ) than brine and with a chemical composition similar to river water. 


\section{- Completion testing}

Once a well has been drilled, engineers and modelers need to be able to estimate the extent and permeability of the reservoir in order to better estimate its future behavior. To do this, completion tests are conducted by varying the flow rate into (or out of) the well, and measuring the resulting pressure response Horne, 1995). At Ngatamariki, completion testing was done with river water $\left(\sim 15^{\circ} \mathrm{C}\right)$ at flow rates of up to $200 \mathrm{t} / \mathrm{h}$.

\section{- Stimulation testing}

Stimulation testing refers to the injection of fluid with the express purpose of measuring the change in the permeability of the well. This is important, as the flow rates during injection testing are usually much lower than those required to run the power plant to its full capacity. In the case of a binary plant (Figure 1.1), where the geothermal fluid circulates through the system in a closed loop, the operator needs to be able to reinject nearly 100 percent of this extracted volume. Therefore, an estimation of the potential of a well to accept increasing volumes of fluid is essential in accurately modeling the feasibility of the entire power scheme once the plant comes online (DiPippo, 2016, Grant and Bixley, 2011).

In high-temperature geothermal reservoirs, stimulation is likely associated with thermal contraction of the walls of the fractures though which fluid is flowing (Grant et al. . 2013), which may manifest as aseismic opening of the fractures (e.g. Guglielmi et al., 2015). However, stimulation may also be related to seismic slip on fractures through which permeability can be either increased or decreased, an effect which has been extensively studied in the lab and through numerical simulations (e.g. Lee and Cho, 2002, Fang et al., 2017, and references therein). See Section 1.5 .2 for further discussion of stimulation and permeability changes.

With these injection scenarios in mind, below we define certain basic terms and quantities that will be used in the following chapters.

Measuring reservoir pressure is of paramount importance to assessing the state of the resource (DiPippo, 2016). This quantity can be measured in the well at or near the depth of the reservoir, in which case the measurement is referred to as downhole pressure (DHP). If the pressure measurement is taken at the wellhead wellhead pressure, WHP , however, some basic corrections need to be made to estimate the reservoir pressure at depth. Assuming a static water column in the well:

$$
W H P=P_{r}-\rho g Z
$$

where $P_{r}$ is the reservoir pressure and $\rho g Z$ is the weight of the water column at depth $Z$. However, if the well is flowing, as is generally the case for wells in a geothermal field (Grant et al., 2013; Clearwater et al., 2015): 


$$
W H P=P_{r}-\rho g Z+\frac{W}{I I}+K W^{2}
$$

where $W$ is mass flow rate (typically measured in tonnes per hour in New Zealand) and $K W^{2}$ is a term accounting for the frictional forces related to fluid flow down the well. II is the injectivity index, typically referred to simply as injectivity, which is a measure of the well's ability to accept fluid at a given pressure. The $I T$ relationship is calculated as (DiPippo, 2016):

$$
I I=\frac{W}{P_{w}-P_{r}}
$$

where $P_{w}-P_{r}$ is the difference in pressure between the well and reservoir (overpressure). The increase in $I I$ is typically referred to as stimulation, which can be thought of as an increase of the permeability of a well. Geothermal operators typically 'stimulate' both production and injection wells once they have been drilled in order to increase the amount of fluid that can be drawn out of (or put into) the reservoir prior to plant startup.

Grant et al. (2013) suggest that the $I I$ of geothermal wells increases as a function of time according to the empirical relationship: $I I=C t^{n}$, where $C$ is a constant, $t$ is time since the start of injection and $n$ has a value between 0.3 and 0.7 for the wells considered. Figure 1.3 shows this relationship during the stimulation of NM08 at Ngatamariki (Clearwater et al., 2015), which stimulated according to $n \approx 0.7$.

Permeability is perhaps the most important property of a geothermal reservoir. It describes the ease with which fluid flows through a medium, and is the primary control on how much fluid can be taken out of, and put back into the reservoir, ultimately determining how much power can be generated (Grant and Bixley, 2011). As defined by Darcy's Law, permeability is DiPippo, 2016):

$$
k=\frac{\nu \mu h}{\Delta P}
$$

where $k$ is the reservoir permeability $\left(\mathrm{m}^{2}\right), \nu$ is the fluid flow velocity through the reservoir $(\mathrm{m} / \mathrm{s}), \mu$ is the dynamic viscosity of the fluid $(\mathrm{Pa} \cdot \mathrm{s}), h$ is the thickness of the reservoir $(\mathrm{m})$ and $\Delta P$ is the applied pressure difference $(\mathrm{Pa})$.

Transmissivity is the permeability thickness product:

$$
T=k h
$$

and describes the capacity of fluid to flow through a reservoir of thickness, $h$. Transmissivity is often more convenient to work with than permeability because $k h$ is easily estimated during standard well-testing operations, whereas the dimensions of the reservoir (i.e. $h$ ) are generally unknown (Horne, 1995).

Hydraulic diffusivity is related to the permeability by: 


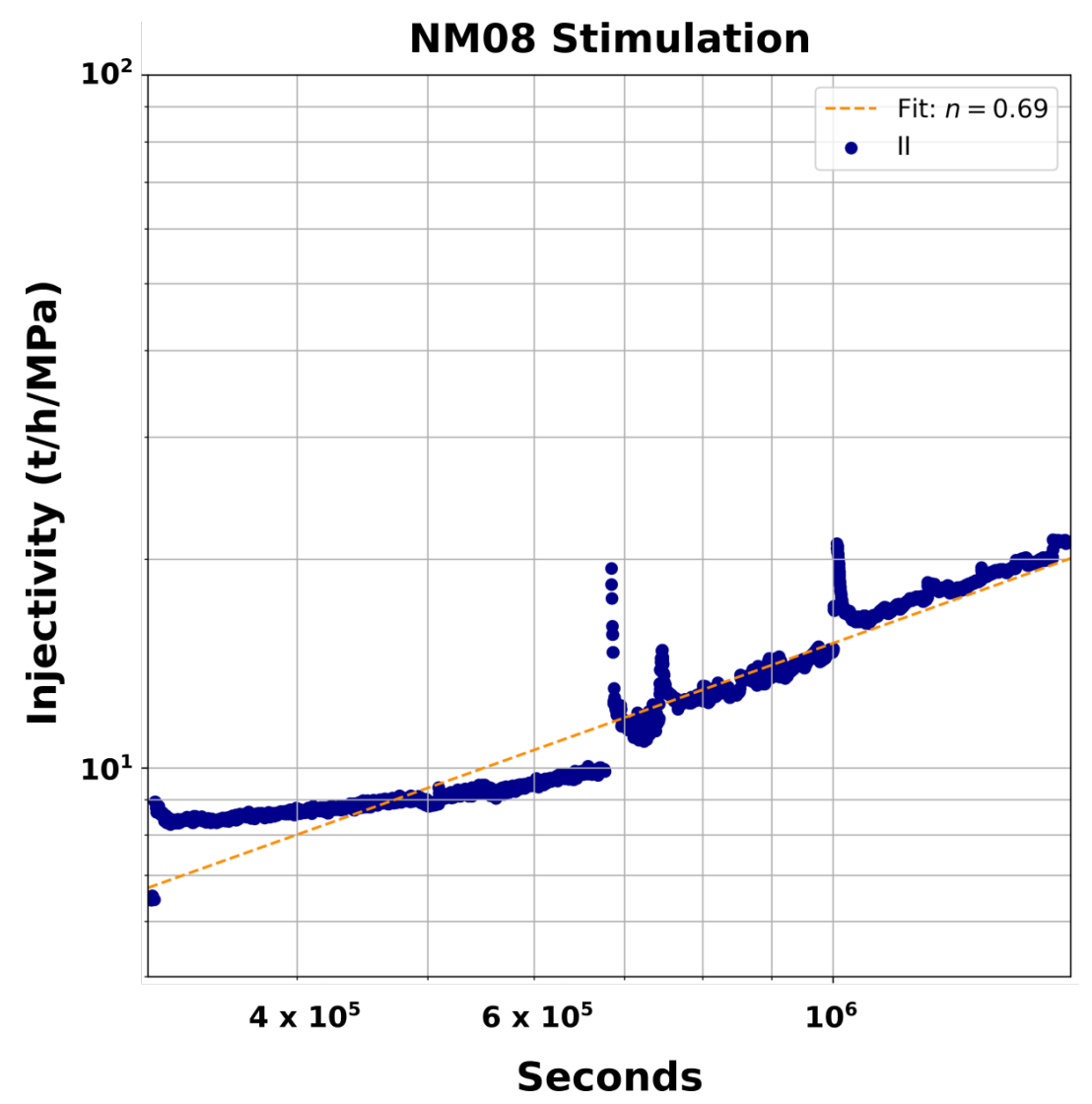

Figure 1.3: Injectivity of NM08 during a portion of the June 2012 stimulation/ completion test. The rate of stimulation agrees with an $n \approx 0.7$ relationship, which is higher than what is typically observed at geothermal wells.

$$
D=\frac{k}{\mu s}
$$

where $D$ is diffusivity, $\mu$ is fluid viscosity and $s$ is the storativity of the reservoir, which is the measure of the volume displaced from a reservoir per unit area and unit reduction in pressure. The diffusion of a pore-fluid pressure perturbation from a well is often thought to correspond to the volume within which induced seismicity is likely to occur (e.g. Shapiro et al., 2002; Parotidis et al., 2004; Shapiro and Dinske, 2009; Shapiro et al., 1997; Jeanne et al., 2014). This use of earthquake hypocenters to infer reservoir properties is known as seismicity-based reservoir characterization (SBRC) (Shapiro et al., 2002) and will be detailed in Section 1.5. However, Riffault et al. (2018) and Cappa et al. (2019) have shown that seismicity may actually occur exclusively outside of the pressurized zone. 


\subsection{Geology, development and conceptual models}

\subsubsection{Taupō Volcanic Zone}

With one exception (Ngāwhā), every producing geothermal field in New Zealand is located in the central North Island. This concentration of high heat flow lies within the Taupō Volcanic Zone (TVZ) (Figure 1.4), an area of backarc extension and volcanism at the margin of the active Hikurangi Subduction Zone where the Pacific Plate subducts obliquely westward beneath the Australian Plate at a rate of $\sim 45 \mathrm{~mm} / \mathrm{yr}$ Cole and Lewis, 1981; Wilson et al., 1995, DeMets et al., 1994, Figure 1.4). In the overriding plate, the rate of extension occurring at the axis of the TVZ ranges from $15 \mathrm{~mm} / \mathrm{yr}$ at the Bay of Plenty to $<5 \mathrm{~mm} / \mathrm{yr}$ near Ruapehu (Wallace, 2004), most of which is accommodated within the Taupō Fault Belt (TFB) (Villamor et al., 2011). Although there is currently some debate as to the extent of the TVZ and how it developed (e.g. Stern and Benson, 2011; Wilson et al., 1995), here we will adopt the interpretation of Wilson and Rowland (2016) where the TVZ is divided into three temporal zones: the old, young and modern as well as three geographic zones: the northern, central and southern (Wilson et al., 1995; Wilson and Rowland, 2016).

The evolution of the TVZ began with the advent of volcanic activity at roughly 2 Ma that continues to the present day. The old TVZ encompasses all identified vents and eruptive centers active during the period from $\sim 2$ Ma until the emplacement of the Whakamaru Group ignimbrites (0.35 Ma), associated with the creation of the Whakamaru caldera (WH, Figure 1.4, Wilson et al., 1995). The young TVZ includes all vents active during the period from 0.35 Ma until the Rotoiti eruption of the Okataina caldera (65 ka; Figure 1.4), and the modern TVZ encompasses the most recent $65 \mathrm{ky}$ of volcanism, with a broadly similar geometry to that of the young TVZ Wilson et al. 1995; Wilson and Rowland, 2016). With the exception of the Mangakino geothermal system, the boundary of the young TVZ encompasses all of the active geothermal systems in the TVZ (black dotted line; Figure 1.4).

The three geographic zones of the TVZ are delineated based on eruptive rates, manifestation and composition. The central TVZ is characterized by a heat output of $\sim 4.2 \mathrm{GW}$ (concentrated almost exclusively at the geothermal fields; Thompson, 1980; Bibby et al., 1995) and $>6000 \mathrm{~km}^{3}$ of Quaternary, rhyolitic volcanism (Wilson et al. 2009, calderas, Figure 1.4). The northern and southern zones (northeast of Kawerau and southwest of Tokaanu, not shown in Figure 1.4) are characterized by andesite-dacite composite cones (larger in the south) and very little rhyolitic or basaltic products. The eruptive rate in the south is less than one third and the heat output one fifth that in the central TVZ (Wilson and Rowland, 2016). These values are poorly constrained in the north, mostly due to the area's subaqueous nature, but the output is likely even less than that in the south (Wilson and Rowland, 2016). 
The central TVZ, where all of the active TVZ geothermal systems are located, is made up of at least eight separate, rhyolite calderas, of which the two most recently active are Taupō in the southwest and Okataina in the northeast (Wilson et al., 1995). The Ngatamariki and Rotokawa systems both lie at the eastern edge of the 0.35 Ma Whakamaru caldera and within the Taupō-Reporoa Basin (TRB; Wilson and Rowland, 2016; Downs et al., 2014, Figure 1.4). Heat flow within the TRB is the highest in the TVZ, as evidenced by the concentration of geothermal systems (Wilson and Rowland, 2016). However, the TRB is sparsely faulted relative to the Taupō Fault Belt to the west, where most of the active extension is accommodated (indicated by the high concentration of faults in Figure 1.4.

\subsubsection{Ngatamariki}

\section{Geology}

The Ngatamariki geothermal field is located approximately $17 \mathrm{~km}$ north of the town of Taupō (Figures 1.4, 1.5). Ngatamariki is a high-temperature, liquid-dominated and naturally-fractured system (with temperatures as high as $280^{\circ} \mathrm{C}$ at depths exceeding $1000 \mathrm{~m}$ ) that measures roughly $5.5 \mathrm{~km}$ from north to south and $3 \mathrm{~km}$ east to west (Bignall, 2009, Chambefort et al., 2014). The reservoir is hosted in volcanic rocks of pre-Whakamaru age, known as the Reporoa Group (old TVZ, $>0.35 \mathrm{Ma}$; Chambefort et al. 2014). In the southern portion of the field, the oldest unit in the Repora Group is the Rotokawa Andesite, which overlies the Torlesse Greywacke basement and, therefore, represents the onset of volcanism in the TVZ Chambefort et al., 2014; Wilson and Rowland, 2016, Figure 1.6. The overlying units are referred to as the Tahorakuri Formation and consist of older andesitic lava flows and younger volcaniclastic units of varying composition, representing $\sim 1$ Mys of volcanism (Chambefort et al., 2014). In the northern part of the field, the upper Tahorakuri volcanic sequence is overlain by a sedimentary succession interpreted to represent a period of relative volcanic quiescence and active tectonism (Chambefort et al. 2014). At that time, the Ngatamariki intrusive complex was emplaced in the northern part of the field, the only such plutonic body yet sampled in the TVZ Chambefort et al., 2014). This $\sim 0.7$ Myr-old tonalite-diorite body defines the northern reservoir at depths of $>2000 \mathrm{~m}$ bsl (below sea level), whereas, in the south, the Rotokawa Andesite is found at these depths Chambefort et al. 2014, Figure 1.6). Overlying the Tahorakuri across most of the field is the Whakamaru Group ignimbrite, erupted from the Whakamaru Caldera (WH, Figure 1.4 Chambefort et al., 2014; Wilson et al., 1995). The younger overlying units represent various periods of volcanism and sedimentation until the deposition of the modern surface deposits Chambefort et al. 2014, Figure 1.6. 


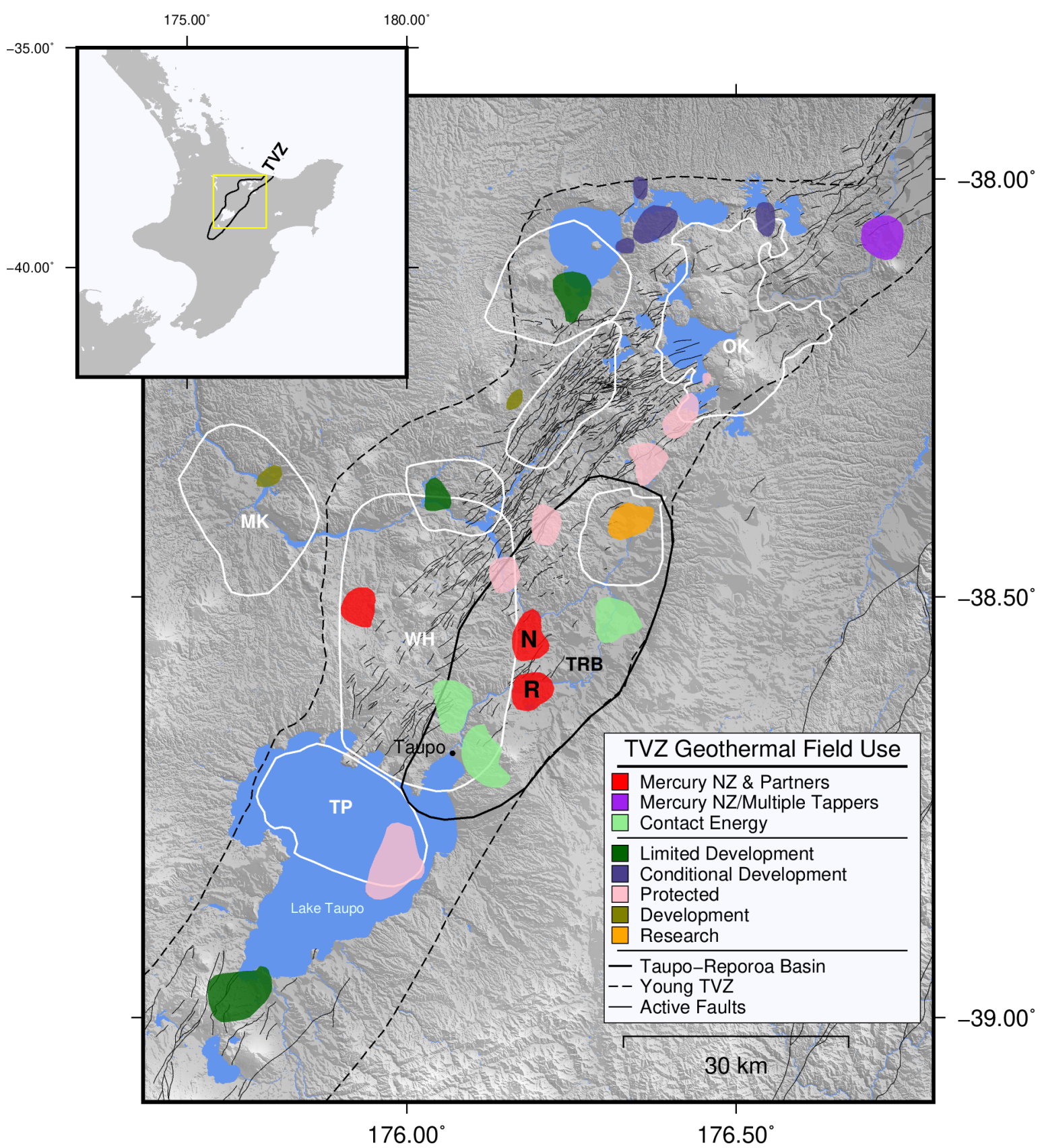

Figure 1.4: The resistivity boundaries of the 23 geothermal fields in the central Taupō Volcanic Zone (after Bibby et al., 1995) colored by degree of development or geothermal operator. Rotokawa and Ngatamariki are labeled ' $\mathrm{R}$ ' and ' $\mathrm{N}$ ', respectively. The boundary of the young TVZ, as defined by Wilson et al. (1995), is shown as a dotted line and their eight identified rhyolitic calderas are outlined in white (WH-Whakamaru Caldera, TP-Taupō Caldera, OK-Okataina, MK-Mangakino). The Taupō-Reporoa Basin (TRB) is outlined in thick black after Downs et al. (2014).

There are a number of mapped (Figure 1.5) and buried (Figure 1.6) normal faults within the Ngatamariki reservoir, defining small horst-and-grabben structures at depth (Bignall, 2009). In the south of the field, the larger of these structures are related to the active NE-SW-striking Aratiatia Fault Zone (Figure 1.5), while in the northern reservoir, faulting is less extensive at the surface (Langridge et al., 2016). The primary 
structural trend in the field is NE-SW, related to the current extensional tectonic regime, with a subordinate NNW-SSE trend that has been attributed to the fabric of the Whakamaru caldera margin (Bignall, 2009, Figure 1.4). This trend is evident from the scale of mapped faults down to centimeter-scale fractures interpreted from borehole image logs in both the northern and southern parts of the field Halwa, 2013a b; Massiot et al. 2012).

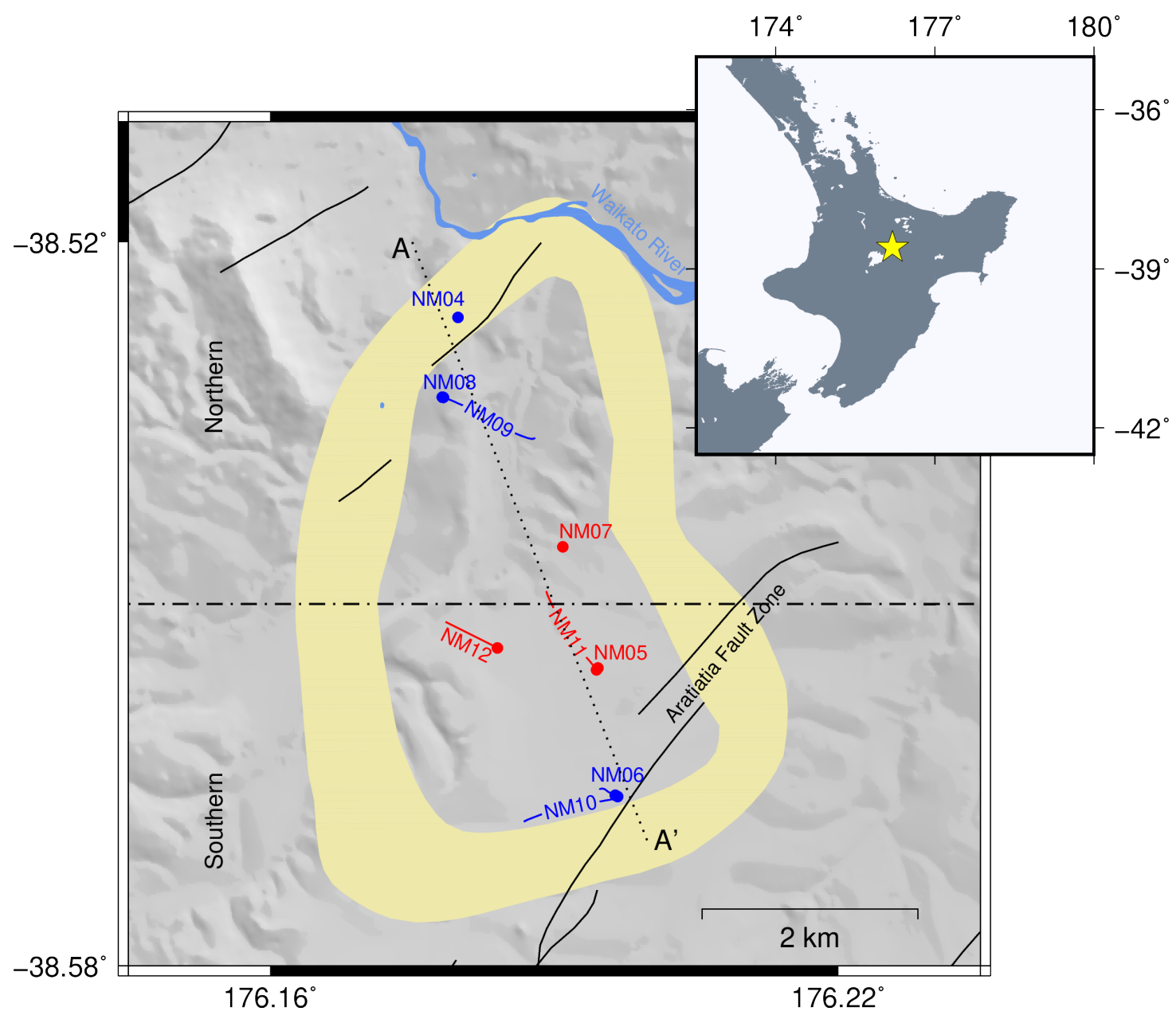

Figure 1.5: Overview of the Ngatamariki geothermal field. Injection wells are shown in blue, production wells in red with dots representing the wellhead and lines showing the surface projection of the well at depth. Wells NM04, NM08, NM05 and NM07 are near-vertical and, therefore, appear only as dots in the figure. Active faults from the GNS Active Faults Database (Langridge et al., 2016) are shown in black. The most likely boundary of the deep resource as described by Boseley et al. (2010) based on magnetotelluric surveys, is shown in yellow. The surface projection of the cross-sections shown in Figures 1.6 and 1.8 is dotted line $\mathrm{A}-\mathrm{A}^{\prime}$. The location of the field on the North Island is shown as a star on the inset map.

\section{Development}

Development at Ngatamariki began with the drilling of four deep exploration wells by the New Zealand government in 1984 (Clearwater et al., 2015). Although these 


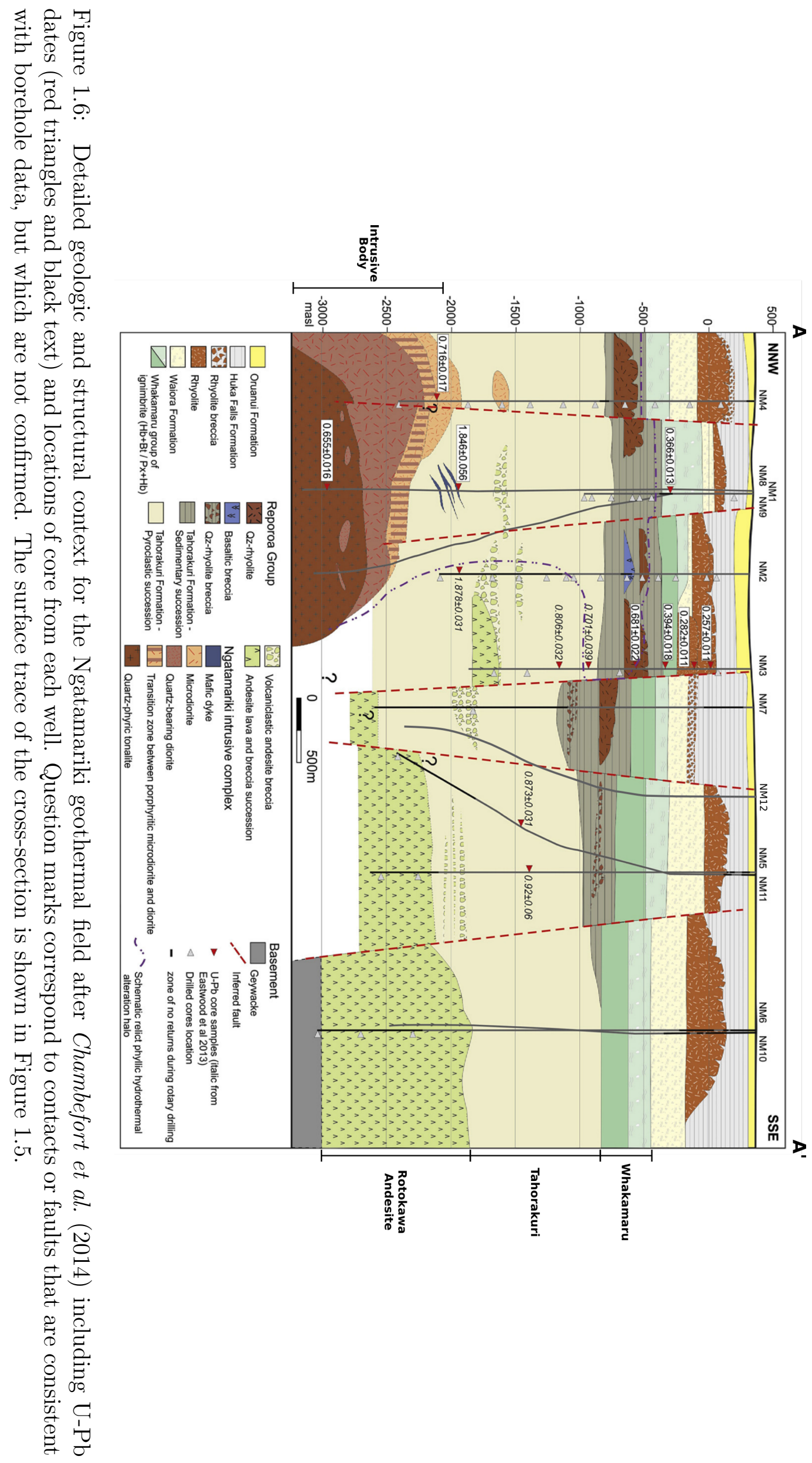


demonstrated the existence of a potentially viable resource, full-scale development for geothermal power production was not undertaken until 2004 when Mercury (then known as Mighty River Power) partnered with the Tauhara No. 2 Trust, representing Māori landowners, to drill additional wells and eventually build the current $82 \mathrm{MWe}$ power station (Clearwater et al. 2015). Between June 2012 and February 2013, NM08, NM09 and NM10 were drilled as injection wells at the periphery of the deep reservoir. For each, a completion/stimulation test was conducted (Clearwater et al., 2015). The Ngatamariki power plant was then brought online starting in April 2013 with gradually increasing flow rates until the reservoir reached a roughly stable state at around the start of 2015 (Figure 1.7).

Mercury have identified three periods between 2012 and 2015 during which the relationship between injection and production parameters and seismicity is of particular interest. We have added the period of NM08 stimulation that was previously analyzed by Matson (2019):

\section{- NM08 stimulation}

From 7 June to 10 July, 2012 (Figure 1.7), northern injection well NM08 (Figure 1.5 underwent a completion and stimulation test. Although low permeability was encountered during drilling, NM08 responded well to stimulation (Clearwater et al., 2015). Matson (2019) analyzed this particular injection using similar techniques to what will be detailed in this work. Here, we also analyze this period and compare these results to those obtained independently by Matson (2019) in order to check our methodology.

\section{- NM09 stimulation}

From December 2012 until late January 2013 (Figure 1.7), northern injection well NM09 (Figure 1.5) also underwent completion and stimulation testing. NM09 permeability (as inferred from injectivity) increased in response to stimulation, although not as strongly as at NM08, and very little seismicity was detected (Clearwater et al., 2015).

\section{- NM10 drilling losses and stimulation}

In July 2012 (Figure 1.7), total fluid losses were encountered during drilling of southern injection well NM10 (Figure 1.5). This was followed, a month later, by a completion and stimulation test, as at NM08 and NM09.

\section{- NM12 drilling losses}

Similar to what occurred at NM10 in 2012, total fluid losses were incurred during the drilling of production well NM12 from June to September 2014 (Figure 1.7).

In addition to these periods of specific interest, we will also investigate the seismic response to the plant startup at Ngatamariki (Figure 1.7), which involved flow rates 
up to five-times greater than during the periods outlined above, and $\mathrm{WHP}$ of up to 2.0 MPa.

\section{Conceptual model}

Hydrologically, Ngatamariki consists of three semi-isolated aquifers at differing depths: the shallow aquifer at the surface, an intermediate aquifer above $\sim 500 \mathrm{~m} \mathrm{bsl}$, and the deep geothermal reservoir below that (Boseley et al., 2010, Chambefort et al., 2016. Figure 1.8). Each of these aquifers is separated by an impermeable layer that, for silicic volcanic rocks in the presence of acidic fluids, is created by alteration of the protolith to illite-smectite clay (Boseley et al., 2010). These layers are normally diagnostic of geothermal reservoirs and are generally inferred from areas of low resistivity (e.g. Bibby et al., 1995) and conductive intervals of well temperature profiles (Grant and Bixley, 2011). The deep reservoir at Ngatamariki is isolated from the intermediate aquifer by a clay cap that diminishes in thickness from $>1000 \mathrm{~m}$ at the northern periphery to a negligible value between monitoring/exploration wells NM2 and NM3, where Boseley et al. (2010) have inferred a hydrologic connection to the intermediate aquifer called 'the leak' (Figure 1.8). This point also marks the center of the 'upflow', which refers to the area of upward convection within a geothermal system and is commonly a target for production wells (Grant and Bixley, 2011). Reservoir permeability in the TVZ is dominated by existing faults and fractures, as the matrix permeability of the major formations is low Grant and Bixley, 2011; Massiot et al., 2015b; Cant et al., 2018). This is certainly the case at Ngatamariki, where the Tahorakuri Formation and Rotokawa Andesite that host the reservoir are shown to be highly fractured in image $\operatorname{logs}$ Halwa, 2013a b; Massiot et al., 2012; Quinao and Zarrouk, 2018).

Nearly all of the fluid produced at Ngatamariki is reinjected into the reservoir. In order to maintain flexibility with regard to reservoir management, there are two reinjection fields (green wells, Figures 1.5, 1.8, blue lines in all later figures) to the north and south of the central production field (blue lines, Figure 1.8, red lines in all later figures). The allocation of injection to each of the two fields varies with time in response to changes in the reservoir and borehole degradation. Generally, more fluid is injected into the northern injection zone than the southern. NM09, in the north, is the dominant injection well for the entire field (currently $>1000 \mathrm{t} / \mathrm{h}$ ). However, permeability heterogeneity is considerable. Well NM08 is far less permeable than NM09, although they are separated by only hundreds of meters at reservoir depth (Clearwater et al. 2015). Permeability also varies substantially with depth, particularly in the northern injection zone where the wells intersect the diorite/tonalite intrusive body, which has a very low matrix permeability (Cant et al., 2018).

While all of the wells drilled at Ngatamariki can be considered to be highly-fractured (Halwa, 2013a, $\mathrm{b}$; Massiot et al., 2012), only a portion of these fractures are open and 

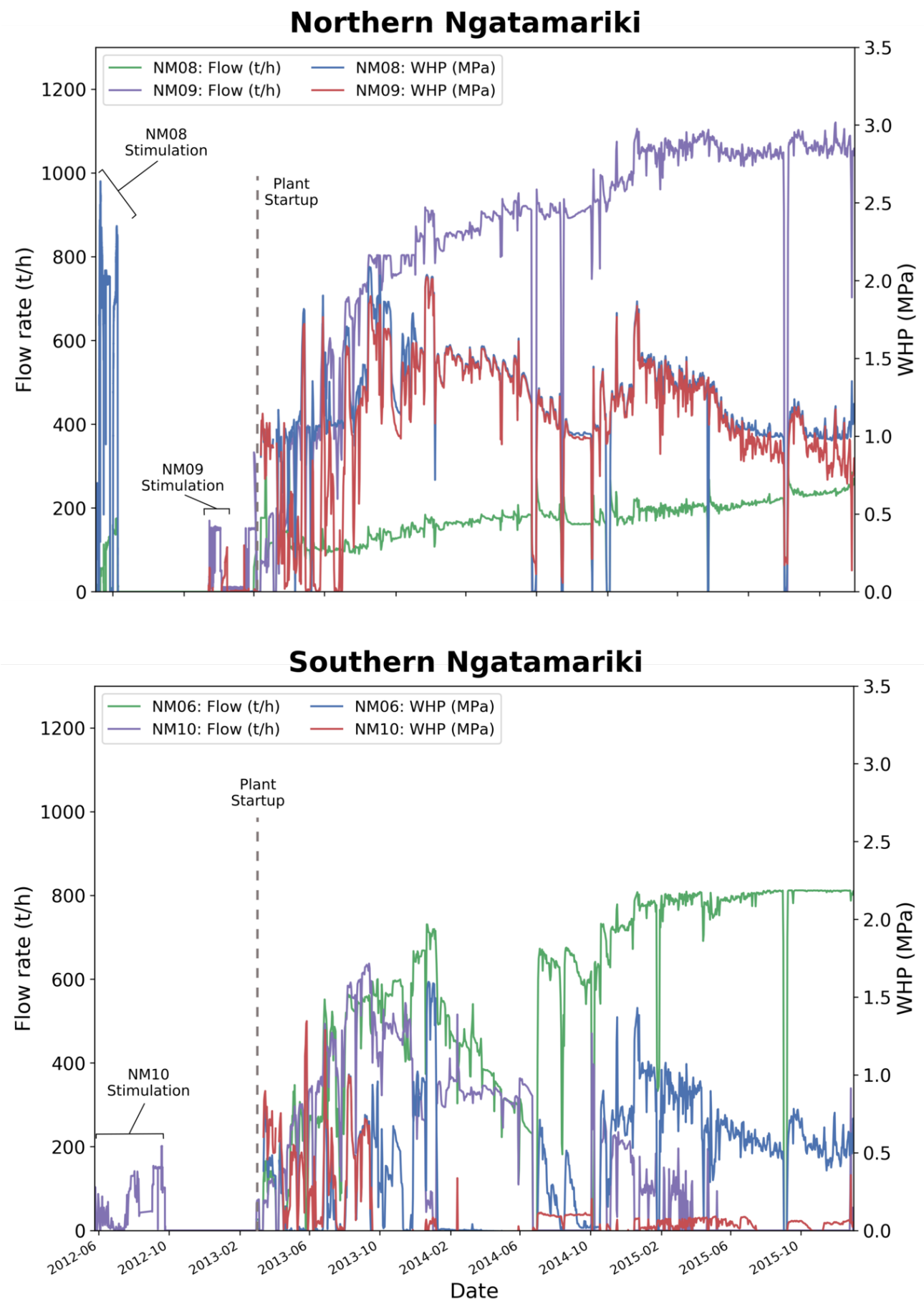

Figure 1.7: Summary figure showing the flow rates and wellhead pressures at each of the four injection wells at Ngatamariki for 2012-2015 (separated into northern and southern injection zones). The main periods of interest, including the three completion/ stimulation tests and the plant startup are annotated. Drilling of NM12 occurred between June and September 2014, but is not annotated here as we have no flow data for that operation. 


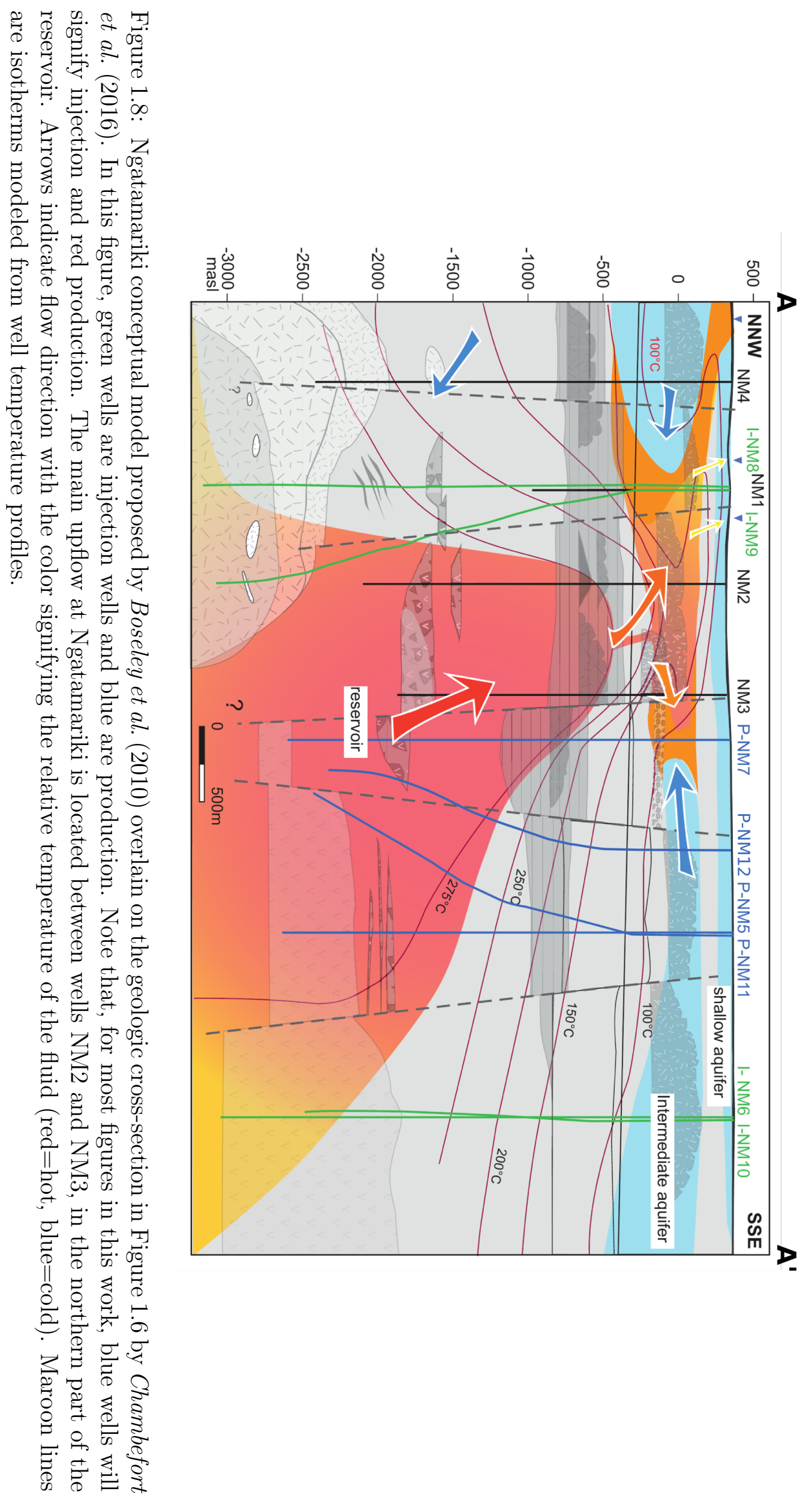


able to accept fluid upon injection or production (we refer to these high-permeability areas as feedzones. Therefore, the most permeable wells in the field are those that intersect active fracture zones that have a high likelihood of being open and not filled with minerals. Such high-permeability fracture zones are intersected at NM09, NM06 and NM10, but not at NM08 (Halwa, 2013ab; Massiot et al., 2012) and these differences are reflected in the wellhead pressures measured at each well.

In the southern injection zone, radioactive tracer tests have revealed well NM10 to be hydrologically connected to the southern production well NM5 Buscarlet et al. 2015). As such a connection produces a loss in entropy (cooler water is extracted), and therefore a loss in power generation capacity at the plant, injection at NM10 was phased out by early 2015 (Figure 1.7). The excess injection in the southern part of the field was then accommodated by NM06 (Figure 1.5).

\subsubsection{Rotokawa}

\section{Geology}

The Rotokawa geothermal field lies $\sim 7 \mathrm{~km}$ south of Ngatamariki. As defined by the resistivity boundaries shown in Figure 1.9 (Risk, 2000), the resource measures approximately $4 \mathrm{~km}$ in diameter. The surface projection of the resource sits astride the Waikato River and all of the development to date has occurred south of the river. Like Ngatamariki, Rotokawa is a naturally-fractured, liquid-dominated reservoir, but the maximum temperatures encountered at depth are the highest measured in the TVZ $\left(338^{\circ} \mathrm{C}\right.$ at $3000 \mathrm{~m}$ bsl in well RK22). The geology at Rotokawa is very similar to that of Ngatamariki, although the heterogeneity of the young TVZ volcanic deposits and active faulting have led to certain differences. Most importantly, while the Ngatamariki reservoir is hosted in Rotokawa Andesite and Tahorakuri Formation rocks, at Rotokawa, much of the reservoir is hosted in the basement Torlesse Greywacke (Figure 1.10: Wallis et al., 2013; McNamara et al., 2016). The rest of the Rotokawa reservoir is mostly hosted in Rotokawa Andesite which reaches thicknesses of $1500 \mathrm{~m}$ and is laterally coherent across the field, although the shallower parts of the production field (red wells, Figure 1.9) are hosted in varying thicknesses of Tahorakuri Formation and Wairakei Ignimbrite (part of the Whakamaru Group, <0.35 Ma age; McNamara et al., 2016). At Rotokawa, there is no analog to the intrusive complex intersected in wells NM04, NM08 and NM09 at Ngatamariki.

The regional NE-SW structural grain describes the first-order structure at Rotokawa, with the Aratiatia Fault Zone cutting across the northwest corner of the field (Figure 1.9) and three NE-SW-striking faults dividing the reservoir at depth: the Production Field Fault (PFF), Central Field Fault (CFF) and Injection Field Fault (IFF) (Figure 1.10 Wallis et al., 2013). The extension of each of these faults has been in- 
ferred from vertical offsets in the basement greywacke and Rotokawa Andesite observed from well cuttings, microseismic locations and radioactive tracer returns between wells (Sewell et al., 2015a; Addison et al., 2017; Wallis et al., 2013), but they are not observed to offset the overlying units (Wallis et al., 2013).

\section{Development}

As at Ngatamariki, resource characterization at Rotokawa began with exploratory drilling in the 1960's by the New Zealand government, which confirmed the existence of the $>300^{\circ} \mathrm{C}$ resource (Sewell et al., 2015a; McNamara et al., 2016). The Rotokawa A RGEN was installed in 1997 with a capacity of 24 MWe. During this phase of development, production and injection both occurred within the modern production field, with injection taking place between 500 and $1000 \mathrm{~m}$ depth (Sewell et al. $2015 \mathrm{a}$ ). In 2000, production was increased to $34 \mathrm{MWe}$ and the shallow injection was moved to deeper wells on the southwest periphery of the production field (e.g. RK18, Figure 1.9). In 2007, the Rotokawa Joint Venture (Tauhara No. 2 Trust and Mercury) were granted consents to increase development at Rotokawa with the addition of the 138 MWe Nga Awa Purua (NAP) triple-flash plant, which was commissioned in 2010 Sewell et al. 2015a). With the drilling of wells RK19-RK30, the management strategy was shifted so that deep injection occurred to the southeast at wells RK20-RK24 and production came from the wells in the already-developed portions of the field (Figure 1.9: Sewell et al. 2015a; McNamara et al., 2016). Requirements for further production prompted the drilling of wells RK32-RK34 following the startup of NAP (Figure 1.9.

During our study period (2012-2015), there were four main periods of interest identified by Mercury from a reservoir management perspective:

\section{- RK24 injectivity decline}

Following NAP startup, it was determined from radioactive tracer returns that the southern injection wells, specifically RK21, were producing rapid returns at the southern production wells Addison et al. 2015). Injection was moved to northern injection well RK24 but within six months $I I$ had begun to decline in the well (Figure 1.11). Mercury have identified this as a time period of interest where patterns of seismicity may be able to inform the reason for this injectivity decline.

\section{- Switch of injection: RK24-RK23}

Given the II decline noted above, Mercury decided to shift some injection away from RK24 and into RK23 (June 2014). It is possible that this produced an accompanying change in seismicity (Figure 1.11).

\section{- RK34 drilling losses}



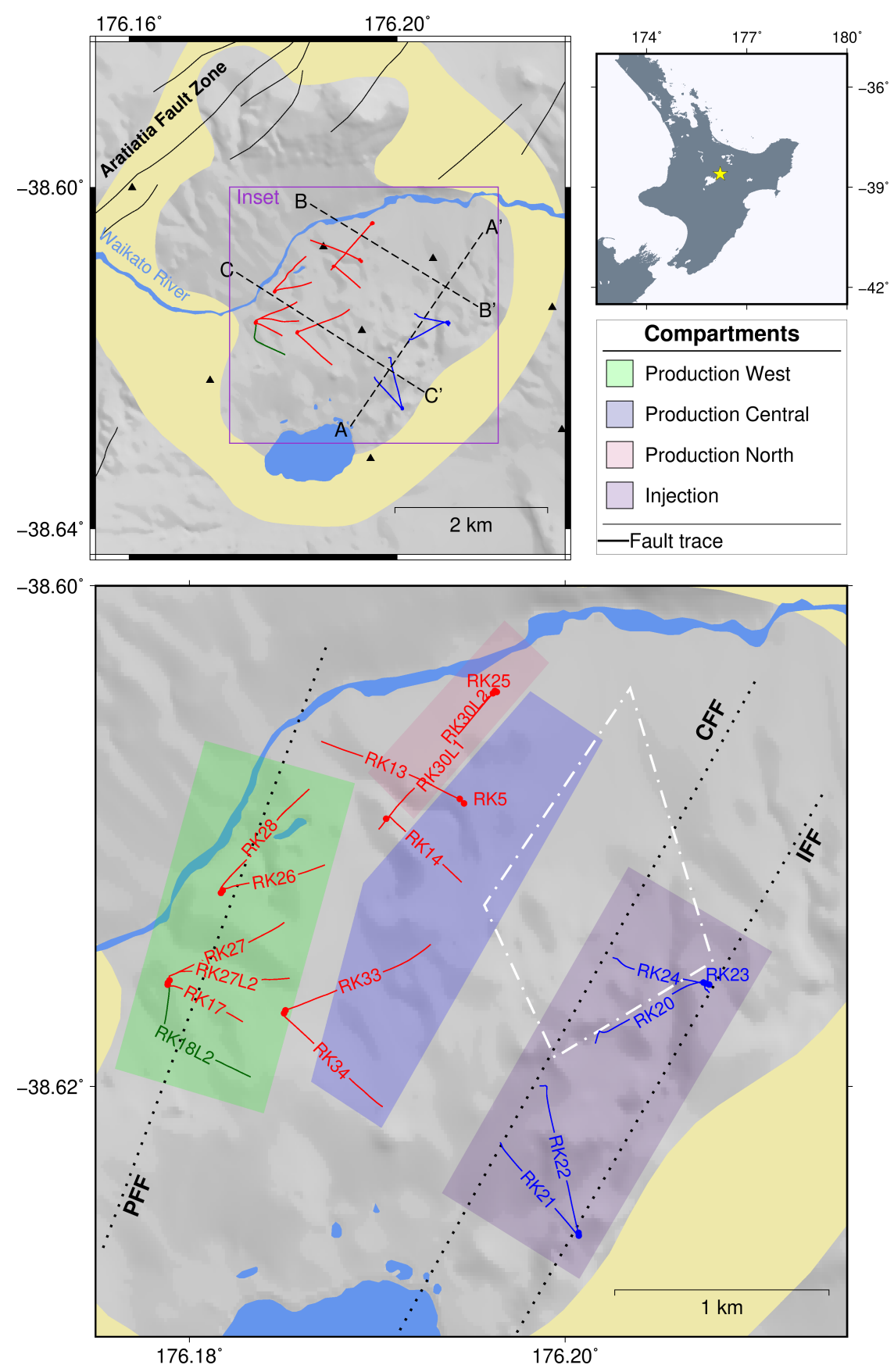

Figure 1.9: Overview of the Rotokawa geothermal field. The top right panel shows the location of the field on the North Island of New Zealand (yellow star). The top left panel shows the resistivity boundary of the field in yellow and injection, production and monitoring wells in blue, red and green, respectively. Solid black lines are active faults. Dotted lines correspond to cross-sections A-A', B-B' and C-C' in Figures 1.10 and 1.12. The lower panel shows a closeup of the field, with the well names labeled and the three known faults $(\mathrm{PFF}, \mathrm{CFF}$, IFF $)$ shown as black dotted lines. The white dot-dashed line shows the extent of significant seismicity from 2008-2012, as reported by Sherburn et al. (2015). There are four known compartments in the Rotokawa reservoir shown as colored polygons. Each is semi-isolated from the others by either a permeability contrast or impermeable barrier (i.e. a fault). The production field comprises three compartments, the west, central and north (green, blue and red, respectively). The injection field is shaded in purple and is a separate compartment. 


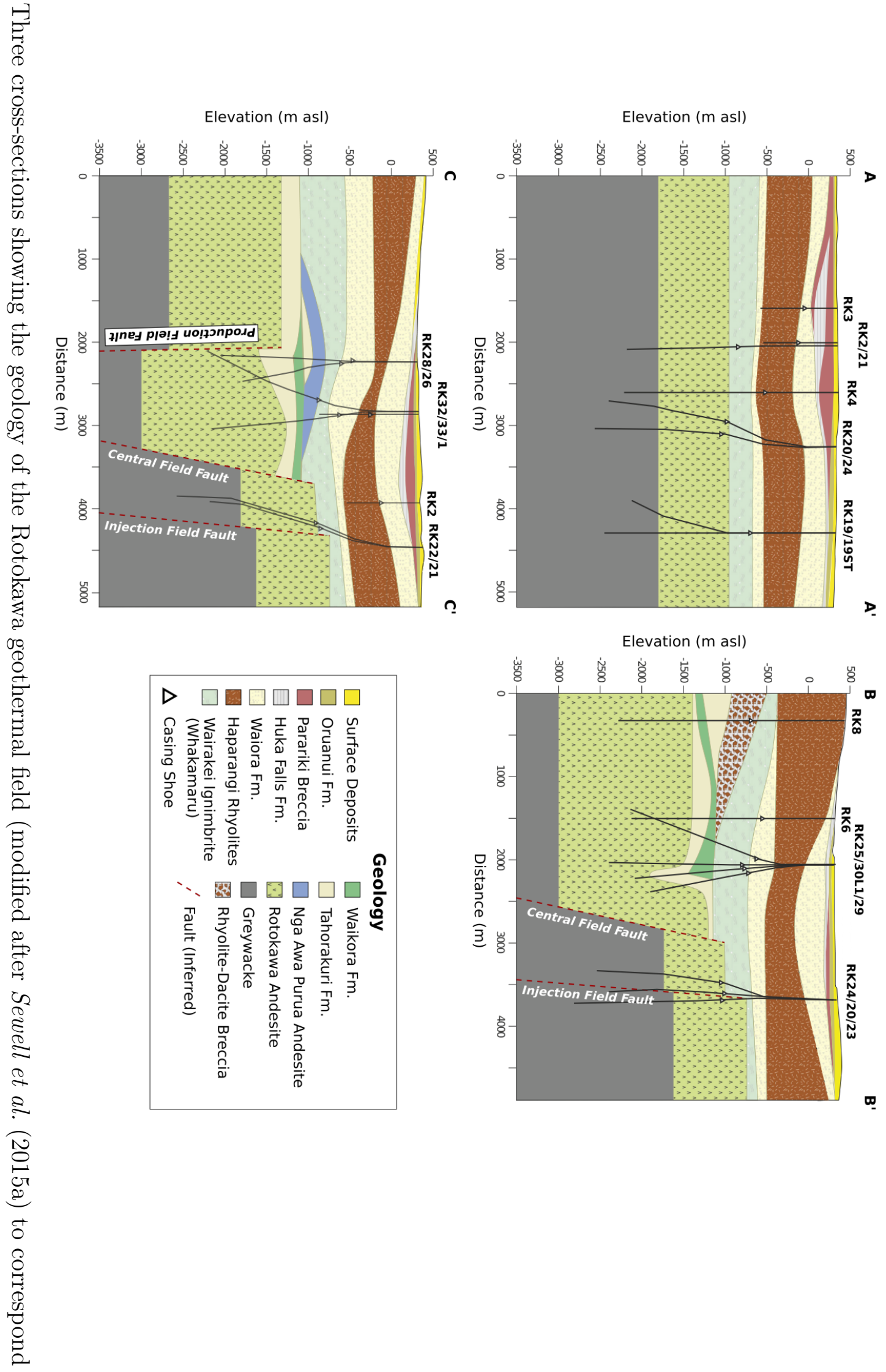


As with NM10 at Ngatamariki, total losses were incurred during the drilling of make-up production well RK34 (September to November 2014). As RK34 was drilled in the seismically-quiet production field, any accompanying seismicity may be more easily identified than in the injection zone.

\section{- Plant shutdowns and startups}

For prior periods (i.e. before 2013), swarms of seismicity may have occurred in response to plant shutdowns and startups (typically for maintenance). These swarms have been attributed to transient pressure changes in the reservoir Sherburn et al. 2015), and Mercury have identified future plant shutdowns as periods of seismic interest.

\section{Conceptual model}

The Rotokawa reservoir has been studied during more than 20 years, spanning two phases of development. As a result, the understanding of the reservoir and the nature of fluid flow is well developed (Sewell et al., 2015a; Addison et al., 2017; Wallis et al., 2013). The hottest temperatures in the field are found in the southern injection zone, with a maximum temperature of $338^{\circ} \mathrm{C}$ measured in well RK22 (Figure 1.9), but there are strong lateral gradients in the natural-state temperatures to the northeast and northwest Sewell et al., 2015a; Wallis et al., 2013) (Figure 1.12). The main upflow of $>330^{\circ} \mathrm{C}$ fluid is located near wells RK21/RK22, although the southwest extent of the reservoir and upflow has not been verified by drilling Sewell et al., 2015a; Winick et al. 2011). Similar to Ngatamariki, Rotokawa is overlain by intermediate and shallow aquifers. However, the hydraulic connection between the deep reservoir and intermediate aquifer at Rotokawa is more pronounced than the 'leak' at Ngatamariki Winick et al., 2011, Figure 1.12.

At Ngatamariki, fluid flowing from injection to production travels $\mathrm{N}-\mathrm{S}$, oblique to the regional NE-SW structure. At Rotokawa, the injection-production flow path is oriented NW-SE, perpendicular to the structural trend. This geometry has implications for fluid flow through the reservoir as faults (in this case) act as along-strike flow pathways and cross-strike flow barriers (Addison et al., 2017; McNamara et al., 2015; Sewell et al., 2015a). The effects of these structural barriers can be seen across the field. The Central Field Fault (CFF) effectively isolates the injection and production zones, allowing only $\sim 12 \%$ of the tracer injected in the injection wells to return to the production field within 60 days (Sewell et al., 2015a; Addison et al., 2015). Similar tracer tests have shown the Production Field Fault (PFF) to be a conduit for rapid along-strike flow within the production field, resulting in the extraction of cool fluid and the shift of deep injection from the southwest production field to the current injection zone (Sewell et al., 2015a; Addison et al., 2017). The Central Field Fault (CFF) 

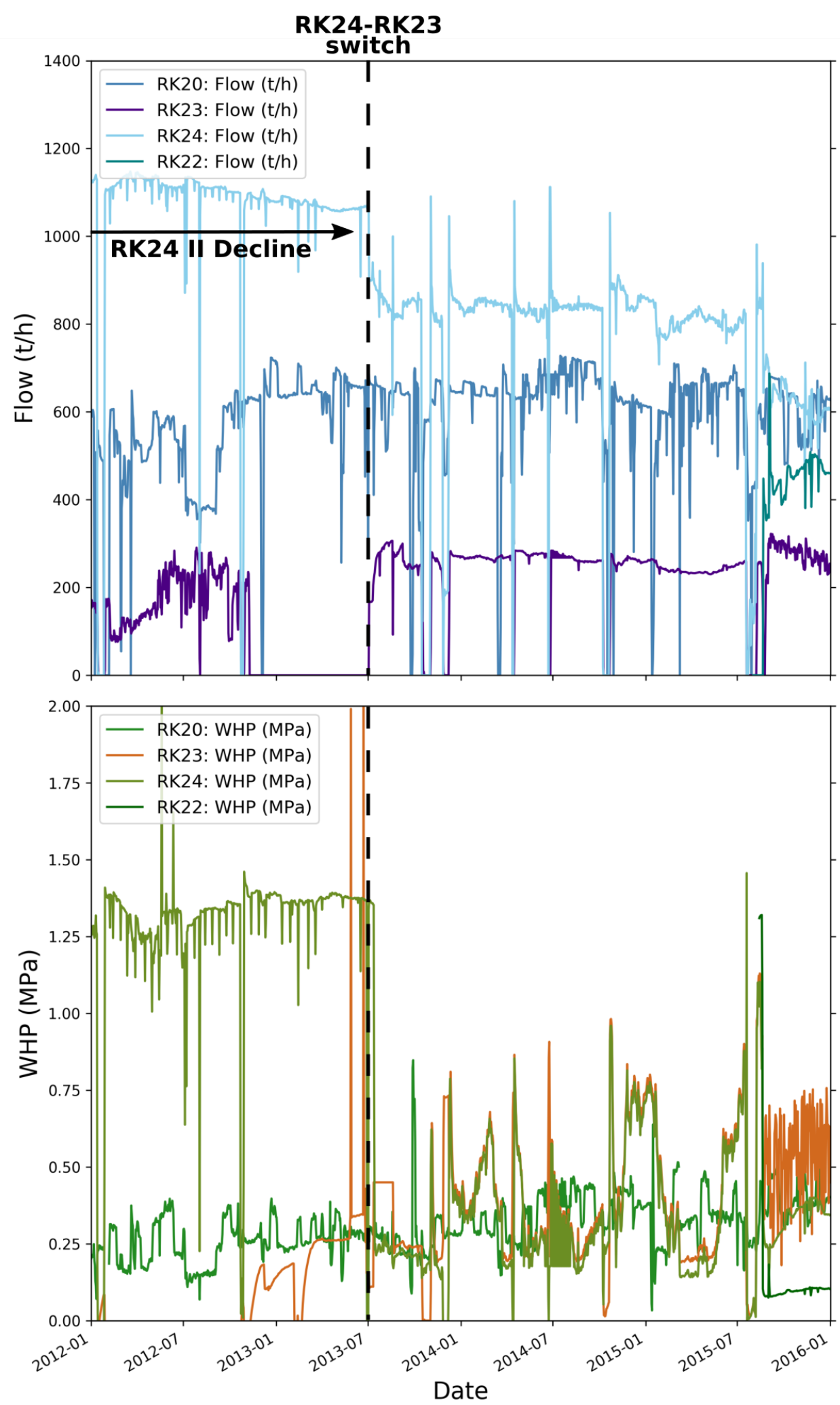

Figure 1.11: Overview of flow rates (top panel) and wellhead pressures (bottom panel) from 2012 until 2015 for deep injection wells at Rotokawa. Selected periods of interest as identified by Mercury are annotated on the flow rate panel. RK34 was drilled from September to November 2014, but is not illustrated here due to a lack of flow rate data. 

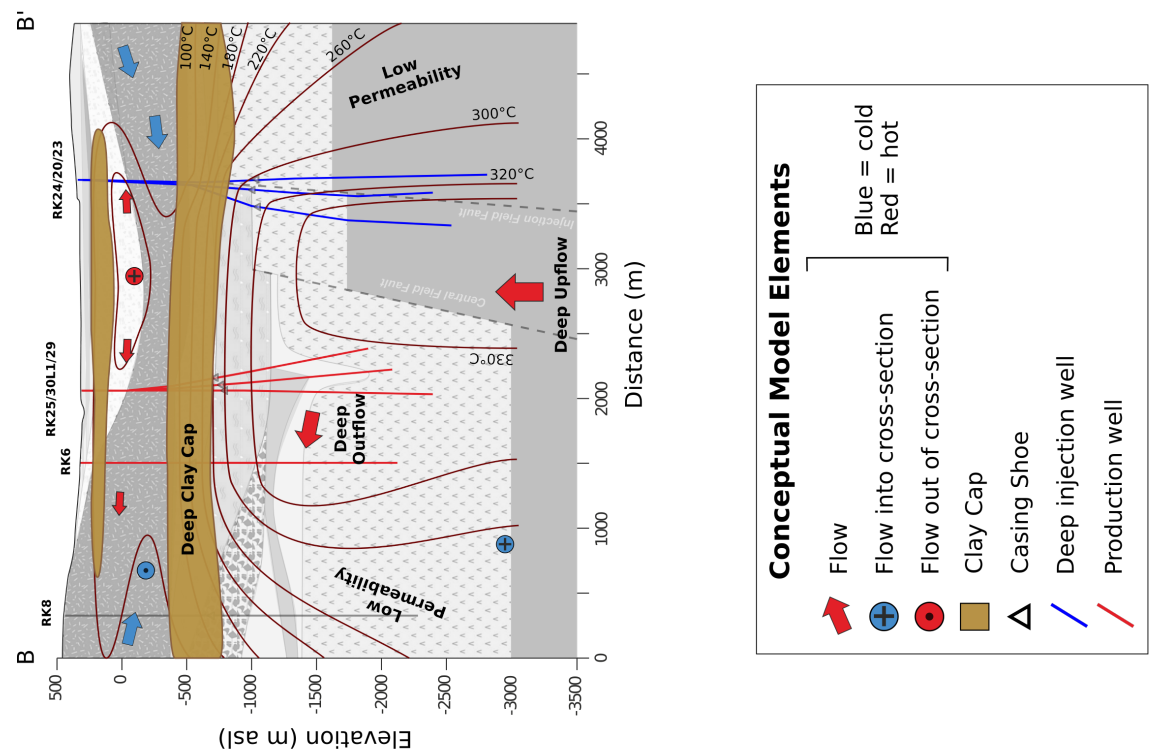

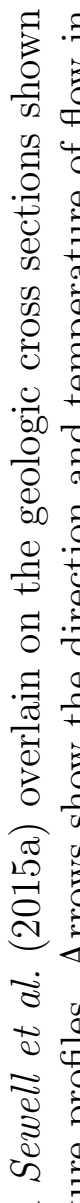

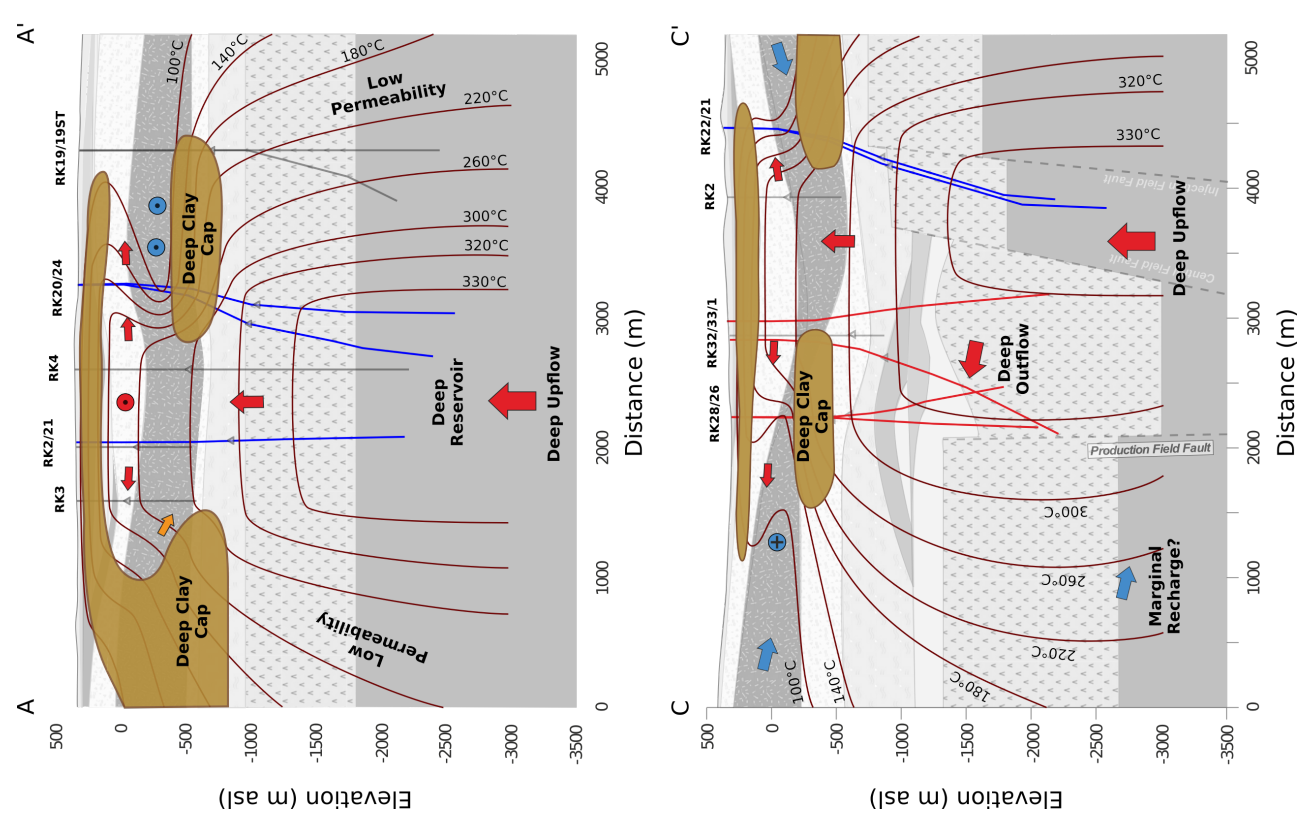

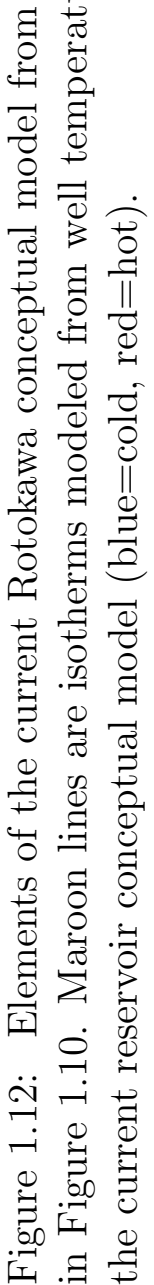


is also inferred to be the structure that controls the connection between the deep and intermediate aquifers, and its surface trace appears to control the locations of a number of features (i.e. hot springs and geysers) at the field (Winick et al., 2011).

Patterns in pressure drawdown in the production field are elongate along-strike, also suggesting that fluid flow is restricted across the structural grain (Hernandez et al. 2015, Sewell et al., 2015a; Quinao et al., 2013). The magnitude of these pressure differentials suggests a high degree of compartmentalization in the reservoir (Figure 1.9. For instance, the pressure drawdown at RK29 since the commissioning of NAP was $\sim 0.4 \mathrm{MPa}$ by 2015 . However, at RK25 and RK30, which are only $\sim 200 \mathrm{~m}$ from RK29, the pressure drawdown is roughly an order of magnitude greater, despite the fact that RK29 extracts more fluid than any other well in the field (Sewell et al., 2015a). Similar NW-SE pressure differentials exist in the southwestern production field and the variations in pressure responses across the entire production field suggest at least three separate compartments (Hernandez et al., 2015, Figure 1.9). The currently-accepted boundaries of the three production field compartments, as well as the injection field, which comprises a separate compartment, are shown in Figure 1.9 .

\section{Seismicity}

Workers at GNS Science and Mercury examine the seismicity at Rotokawa on a yearly basis in an effort to monitor changes in the reservoir. However, the last detailed characterization was only conducted through 2012 Sewell et al., 2015b; Sherburn et al., 2015). As expected, seismicity migrated in conjunction with the evolving injection strategy. At the end of 2012, with injection occurring predominantly into the southeastern compartment of the reservoir, Sewell et al. (2015b) and Sherburn et al. (2015) observed that seismicity occurred inside the white dotted diamond shown in Figure 1.9. migrating from the southern half of the diamond in 2008 to the northern half by 2012 . The location of the northwest segment of this diamond has also been used to infer the location of the CFF. In addition, Sewell et al. (2015b) and Sherburn et al. (2015) concluded that seismicity at Rotokawa was induced mostly by stress changes associated with reservoir cooling and contraction, instead of by pore-pressure increase, due to the relatively small pressure buildup measured at the wells $(<1.5 \mathrm{MPa})$. In the analysis that follows, specifically in Chapters 4 and 5, we look to update this understanding of Rotokawa seismicity.

\subsection{Induced seismicity}

Human-induced seismicity was first recognized decades ago, but has received a large amount of attention recently due to a remarkable increase in the number of events in the central United States and elsewhere Ellsworth, 2013; Kim, 2013; Keranen et al., 
2013; Zoback and Gorelick, 2012; Baisch et al., 2006; Evans et al., 2004, Deichmann and Giardini, 2009; Langenbruch and Zoback, 2017; Kim et al., 2018; Grigoli et al., 2018; Ge et al. 2019). Human-triggered seismicity has been observed for nearly a century in relation to the impoundment of large water reservoirs (e.g. Carder, 1945; Gupta, 2018) and early oil and gas operations (Hough and Page, 2016). Following a high-profile case of induced seismicity at the Rocky Mountain Arsenal near Denver, Colorado in 1962 Healy et al. 1968), a controlled experiment at the Rangely oil field in Colorado demonstrated a convincing connection between fluid injection and seismicity Raleigh et al., 1976). Also, starting in the 1960s, new hydraulic fracturing (i.e. 'fracking') techniques were being used to rehabilitate old oil and gas reservoirs, but these operations garnered little attention as the volumes injected were relatively small, the targeted formations were relatively permeable and the operations produced little seismicity. In the 1990s, however, the combination of massive hydraulic fracturing and advances in horizontal drilling made possible fracking in tight, shale reservoirs and opened large parts of the aseismic interior US to large-volume fluid injection. The rapid deployment of these techniques in places such as Oklahoma, Ohio and Texas brought about noticeable increases in felt seismic events in these areas Ellsworth, 2013) that have since been attributed to the injection of fracking wastewater and to fracking itself (e.g. Kim, 2013; Keranen et al., 2013). These events heightened international attention for the topic of induced seismicity and spurred a continuing effort to understand what factors determine where and when injection operations will induce seismicity (e.g. Goebel and Brodsky, 2018).

\subsubsection{Settings of injection-induced seismicity}

Human-induced seismicity is related to a number of processes including mining or gas-related mass extraction and weapons testing (e.g. Chambers et al., 2015, Kim and Richards, 2007). Here, however, we focus on induced seismicity related to the injection of fluids into the subsurface, which generally occurs in three settings:

\section{- Oil and gas extraction}

As mentioned above, the bulk of the attention devoted to induced seismicity internationally is related to enhanced oil and gas recovery through massive hydraulic fracturing. Certain cases have been directly attributed to the hydraulic fracturing treatments themselves (e.g. Atkinson et al., 2016; Skoumal et al., 2015, in Canada). However, the vast majority of cases are induced by the deep disposal of fracking-related wastewater at or below the depths of the fracking wells (e.g. Kim, 2013; Keranen et al., 2013; Yeck et al., 2017; Mc Garr and Barbour, 2017). Many of these events are located at depths below that of the injection and occur on preexisting basement faults up to tens of $\mathrm{km}$ away from the injection well. 
The factors that determine whether seismicity will occur for a given scenario, and what the frequency and magnitude might be, are still a topic of active research.

\section{- $\mathrm{CO}_{2}$ sequestration}

Over the past decade, the possibility of capturing $\mathrm{CO}_{2}$ produced by industrial activities and reinjecting it into the subsurface has been raised as a potential strategy for mitigating human-induced climate change. As a result, a number of pilot projects exploring the efficacy of carbon capture and storage have been undertaken in various locations worldwide (Evans et al., 2012; Zoback and Gorelick, 2012). This process involves the injection of liquid (supercritical) $\mathrm{CO}_{2}$ into the subsurface, and therefore may generate seismicity via the same processes involved with fracking and wastewater disposal National Academy of Sciences, 2013). Currently, the future risk of induced seismicity associated with these projects is difficult to determine. If this technology is to be adopted as a viable climate-change mitigation strategy, it will involve injected volumes many orders of magnitude larger than those related to the current pilot projects (Zoback and Gorelick, 2012). However, even at these relatively small volumes, significant seismicity has been recorded in some locations (e.g. Goertz-Allmann et al., 2017).

\section{- Geothermal power production}

As described above, power can be produced by 'mining' heat from deep reservoirs in the earth's crust. These reservoirs can be divided into enhanced geothermal systems (EGS) and conventional reservoirs. In this thesis, we deal with conventional reservoirs. However, the seismicity induced at EGS reservoirs is still relevant to the discussion of injection-induced seismicity, especially in medium to high-temperature reservoirs.

The aim of EGS is to mine heat from hot, yet relatively impermeable, rock at drilling-accessible depths. Usually, this is accomplished through injection of fluids at pressures sufficient to fracture rock (similar to oil and gas fracking) and/or shear preexisting faults and fractures in the reservoir (sometimes called 'hydroshearing'), thereby creating a permeable reservoir between an injection and production well (or wells). Enhanced geothermal systems originated as part of the Hot Dry Rock experiment conducted by the Los Alamos National Laboratory in the 1970's National Academy of Sciences, 2013). Subsequent projects were undertaken in England, France, Germany and Japan, with varying degrees of success and in the last 15 years other projects have been undertaken in Australia, Sweden and Switzerland. In each of these cases, some amount of seismicity was induced (e.g. National Academy of Sciences, 2013; Baria et al., 1989, Baisch et al., 2006, Evans et al., 2004), and in two high-profile cases, one at Basel, Switzerland (Deichmann and Giardini, 2009) and the other at Pohang, South 
Korea (Kim et al., 2018; Grigoli et al., 2018; Ge et al., 2019), large-magnitude induced earthquakes have damaged infrastructure and injured people.

Conventional geothermal reservoirs are those which already have sufficient heat and permeability for a convective hydrothermal system to have developed Grant and Bixley, 2011). In these cases, extraction of fluid for power production is relatively easy, as no permeability need be created. However, extraction of fluid can also deplete the resource, causing surface subsidence and pressure drawdown Grant and Bixley, 2011). Fluid is therefore reinjected into the reservoir as a means of pressure support and as a mitigation strategy against resource depletion. Conventional reservoirs are mostly located in areas of active tectonics such as New Zealand, Iceland, Italy, the Philippines, Indonesia, California and Central America and have been associated with seismicity for decades (e.g. Batini et al., 1985; Allis, 1982). Rotokawa and Ngatamariki are both high-temperature, fluiddominated versions of the conventional geothermal reservoir and so are most directly comparable to these types of fields elsewhere.

\subsubsection{Mechanisms of induced seismicity}

Slip occurs on a fracture or fault when the shear force acting upon it exceeds its frictional strength (Stein and Wysession, 2003). This condition can be met through an increase in the shear stress or a decrease in the frictional strength, both of which are dependent upon the orientation of a fracture with respect to the local stress state, described by the principal stress axes $\sigma_{1}, \sigma_{2}$ and $\sigma_{3}$ in order of decreasing magnitude, respectively. The conventionally-accepted mechanism by which seismic slip is induced during fluid injection operations is the increase in pore fluid pressure (Figure 1.13), which decreases the normal stress on the fracture, thereby reducing its frictional strength and allowing a rupture to nucleate (e.g. Majer et al., 2007). However, as has become more clear with the growing number of case studies, there are other process that play important, or even dominant, roles in induced seismicity and interact in ways that are complex and poorly understood (e.g. Guglielmi et al., 2015; Riffault et al., 2018; Cappa et al., 2019). The key mechanisms that induce seismicity during fluid injection include:

\section{- Pore-fluid pressure perturbation}

The simplest explanation for the occurrence of induced seismicity is pore-fluid pressure increase within a fracture network that is hydraulically connected to the injection well. In such a scenario, any pressure perturbation at the well diffuses through the fracture network at a rate that depends on hydraulic properties of the reservoir, specifically hydraulic diffusivity, $D$ (Section 1.3.2). Shapiro et al. (2002) developed a technique called seismicity-based reservoir characterization (SBRC) 
by which the hypocentral locations of induced events could be used to infer the hydraulic diffusivity of a reservoir. This technique is based on the assumption that induced seismicity is induced purely as a result of the pore-fluid pressure increase, originating at an injection well and diffusing outwards. This phenomenon is sometimes referred to as the slow 'Biot wave' (Biot, 1956). The outward diffusion of fluid molecules defines an envelope of $r=\sqrt{4 \pi D t}$ for homogeneous, isotropic media, within which elevated pore pressure decreases the effective normal stress acting on a fracture network. As a result, the entire network is brought closer to failure and seismicity is likely to occur, especially on structures that were already critically-stressed (Townend and Zoback, 2000). This technique has been shown to be effective at describing the spatio-temporal occurrence of seismicity for numerous injections (e.g. Shapiro et al., 1997; Shapiro and Dinske, 2009; Parotidis et al., 2004; Jeanne et al., 2014, Figure 1.14. However, it must be noted that this approach ignores the possibility of triggering by poroelastic stress transfer or thermal stress changes.

Recently, however, Cappa et al. (2019) have shown that elevated fluid pressures may actually encourage rate-strengthening behavior in a fault zone. If this is indeed the case, the zone affected by pore-fluid pressure increase does not correspond to the seismically-active zone at all (as has also been suggested by Riffault et al., 2018), and seismic slip (requiring rate-weakening behavior) presumably occurs due to stress transfer by stable (aseismic) sliding onto fractures further afield. This finding may require rethinking SBRC, specifically, and pore-pressure increase as a triggering mechanism more generally.

\section{- Poroelastic stress transfer}

In addition to the perturbation of pore-fluid pressure, removal or injection of large amounts of fluid from the subsurface induces elastic stress changes due to the volume change of the reservoir (National Academy of Sciences, 2013). In the case of depleted oil and gas reservoirs, or certain geothermal fields, this process can involve compaction of the reservoir and induce slip either within or outside the reservoir, depending on the prevailing tectonic regime Segall et al., 1994; Segall and Fitzgerald, 1998). Where fluid is being injected into the reservoir, the corresponding increase in volume also induces a stress change. In the nearfield (100s of $\mathrm{m}$ ), the pore-fluid pressure increase experienced by fractures that are hydraulically connected to the injection well dominates the stress induced by volume change (National Academy of Sciences, 2013). However, poroelastic stress transfer has been shown to dominate at greater distances and therefore, should not be ignored in assessing the effect of injection on induced seismicity Goebel et al., 2017). Recently, Goebel and Brodsky (2018) suggested that the degree to which either pore-pressure changes or poroelastic response dominate the 


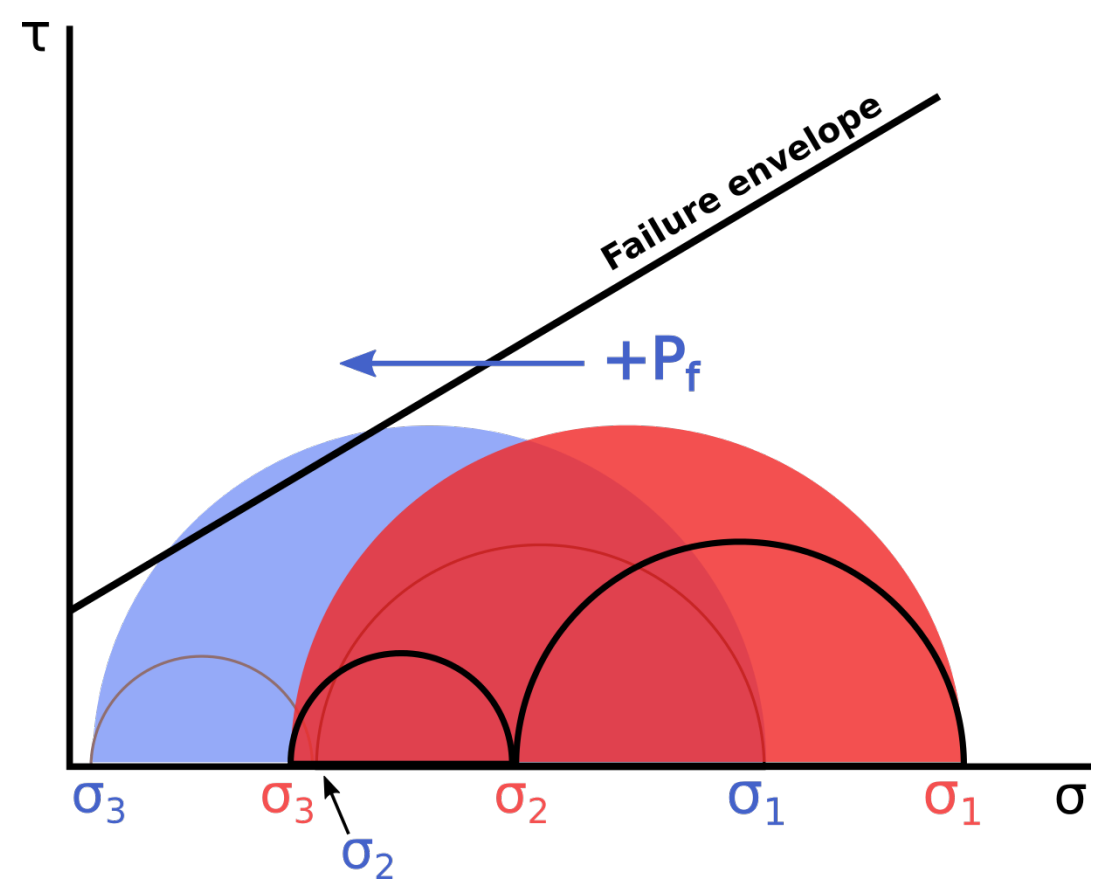

Figure 1.13: For a given stress tensor, the 3D Mohr's diagram comprises three circles, the largest of which has a diameter equal to $\sigma_{1}-\sigma_{3}$ (differential stress) and the two inner circles have diameter $\sigma_{1}-\sigma_{2}$ and $\sigma_{2}-\sigma_{3}$, from right to left. Any given plane can be plotted as a point above the smaller circles and below the largest according the normal $(\sigma ; \mathrm{x}$-axis $)$ and shear $(\tau ; \mathrm{y}$-axis) tractions acting on it. When this point plots above the Mohr-Coulomb failure envelope (the arbitrarily-sloping black line), the plane is in disequilibrium and will fail. Here we show an unperturbed stress tensor in red, and the same tensor when a positive change in the pore-fluid pressure $\left(\mathrm{P}_{f}\right)$ is applied (blue). This has the effect of equally lowering the magnitudes of each principal stress, shifting the Mohr's circle to the left and, in this case, resulting in a population of planes above the failure envelope that are suitable for failure (if they exist).

seismic sequence is determined by the depth of injection with respect to basement. In their study, sub-basement injections were dominated by pore pressure increases and the associated seismicity terminated abruptly away from the well, whereas above basement injections were dominated by poroelastic stress transfer, had much wider spatial footprints and triggered larger magnitude events for a given injected volume (Goebel and Brodsky, 2018). However, Hincks et al. (2018) modeled the correlations between induced seismicity and a large number of operational and geological parameters for induced seismic sequences in Oklahoma and found a significant negative correlation between above-basement depths and seismic moment release.

An additional consideration for any seismic sequence, whether natural or induced, is the static (Coulomb) stress transfer associated with slip (Okada, 1992). Coulomb stress change is known to explain the migration of aftershock sequences (e.g. Toda, 2003). It has also been shown to affect sequences of induced seis- 
a)

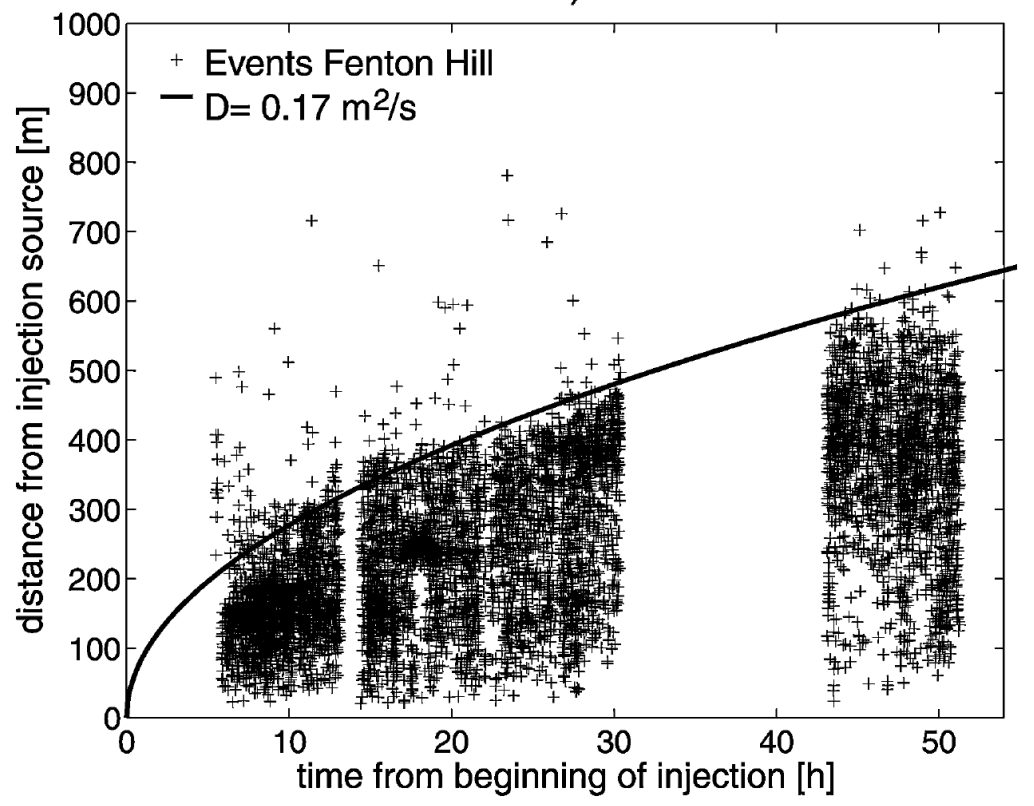

b)

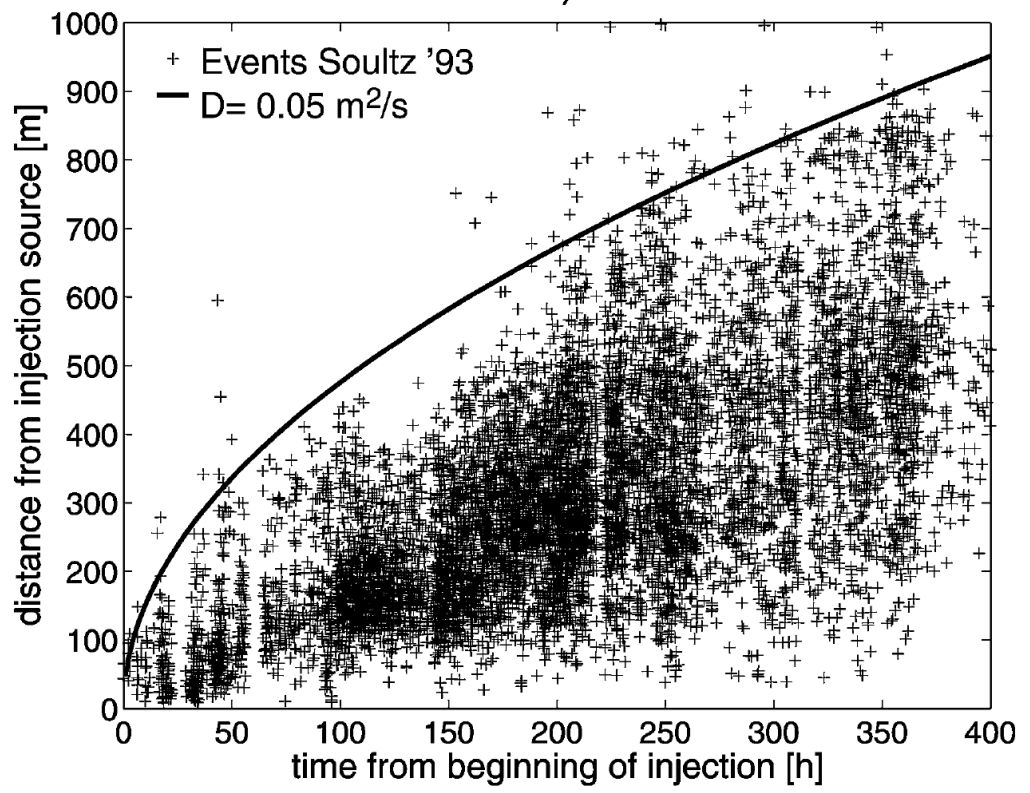

Figure 1.14: Typical 'r-t' plots from Shapiro et al. (2002) showing the triggering front of seismicity as defined by SBRC for two injection operations: a) the Fenton Hill, New Mexico Hot Dry Rock experiment and b) the Soultz EGS project in France. Both sequences are well described by homogeneous, isotropic triggering fronts.

micity during EGS projects (Catalli et al., 2016, Schoenball et al., 2012). As mentioned above, modeling, lab and decameter-scale experiments recently reported by Cappa et al. (2019) and injectivity modeling by Riffault et al. (2018) suggest that coulomb stress transfer may actually be the dominant mechanism responsible for induced seismic slip away from the wellbore, with elevated pore pressures inducing stable, aseismic sliding near the wellbore. 


\section{- Thermoelastic stress transfer}

Finally, particularly when considering induced seismicity in geothermal reservoirs, thermoelastic effects play an important role (Grant et al., 2013). A number of researchers, working at The Geysers geothermal field in northern California, have shown that injection-related cooling of the reservoir can induce large changes in the stress state that affect the style and location of induced faulting (Martínez-Garzón et al., 2014, 2013, 2017; Jeanne et al., 2014, 2015a). This effect is less pronounced, or negligible for injection scenarios where the temperature contrast between the reservoir and injected fluid is small, but in the case of high-temperature geothermal fields (including Rotokawa and Ngatamariki), the contrast can be $200-300^{\circ} \mathrm{C}$ (e.g. Martínez-Garzón et al., 2014, Jeanne et al., 2015a).

Figure 1.15 illustrates the effect of thermoelastic stress reduction on the stress state in a reservoir from a Mohr-Coulomb perspective. The key point, as explained by Jeanne et al. (2014, 2015a), is that the stress reduction is directly related to the shape of the cooled zone, which has a long dimension parallel to the dominant direction of the fluid flow. The longest dimension of the cooled zone is the axis of maximum contraction of the reservoir and therefore the axis of maximum stress reduction. The resulting effect on the reservoir stress state depends on the relationship between this axis of maximum contraction and the orientations of the principal stress components. In the case of cold-water injection into a hot resource, the initial flow from the well will be gravity driven (due to the density of the cold fluid). This will create a vertically elongate cooled zone and therefore will preferentially reduce $\sigma_{V}$ (vertical stress). In the case of The Geysers and most TVZ geothermal fields, $\sigma_{V} \approx \sigma_{1}$ and so cold water injection would have the effect of reducing $\sigma_{1}$, thereby reducing differential stress and stabilizing the reservoir fracture network (Figure 1.15, green circle). Jeanne et al. (2014) were able to show that this effect acted to suppress seismicity within $\sim 200 \mathrm{~m}$ of the wellbore during the EGS demonstration in the northwest Geysers in 2010. However, once the injected fluid begins to migrate laterally, the reservoir also begins to contract laterally and the flow-parallel horizontal stress component is preferentially reduced. If this component happens to be $\sigma_{3}$, then the reservoir differential stress is increased and the fracture network is brought closer to a critically-stressed state (Figure 1.15, blue circle).

\subsubsection{Permeability and seismicity}

For most industrial applications of fluid injection, formation permeability is of paramount importance. Generally, fluid injection operations either seek to increase permeability 


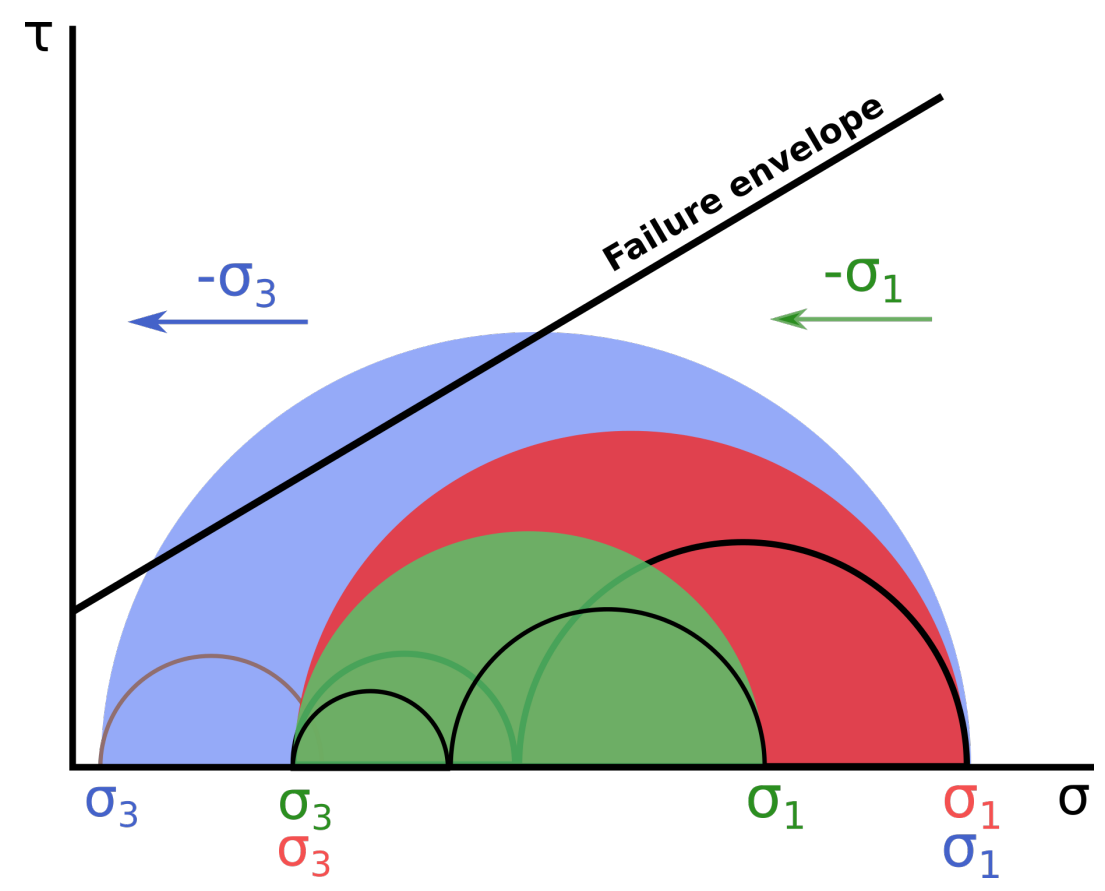

Figure 1.15: Schematic illustration of the changes in the Mohr's circle induced by thermoelastic stress changes in a reservoir due to the injection of cold fluid. The red circle represents the undisturbed state. The induced change in the reservoir stress state is dependent upon the shape of the cooled zone as it relates to the directions of the principal stress axes. Cooling preferentially decreases the magnitude of stress parallel to the dominant direction of flow (and hence the longest axis of the cooled zone) (Jeanne et al. 2014). The blue circle illustrates the effect of fluid flow parallel to $\sigma_{3}$, which would increase the differential stress (defining the diameter of the blue circle) and bring the fracture network closer to a critical stress state. If fluid flow is parallel to $\sigma_{1}$, then differential stress is decreased and the fracture network is made more stable. In the normal-faulting stress state of the TVZ $\left(\sigma_{1} \approx \sigma_{V}\right)$, the blue circle would correspond to a preferred NW-SE flow direction, whereas NE-SW flow would reduce $\sigma_{2}$, a state thath would be represented by a Mohr's circle equal in size to the red circle, but with modified sizes of the subcircles. This would signify a change in the population of fractures which would be able to fail.

(e.g. geothermal well stimulation, hydraulic fracturing) or require that sufficient permeability exists that the formation will accept the fluid being injected (e.g. wastewater disposal, $\mathrm{CO}_{2}$ sequestration, enhanced oil recovery) (National Academy of Sciences, 2013). Induced seismicity, as it relates to any given injection, can be viewed in two lights: as a risk to be mitigated, or as a tool for tracking changes in the reservoir. When viewing induced seismicity as a potential risk, it must be weighed against the goals of the project, which often include increasing the formation permeability (e.g. fracking or geothermal stimulation). In these cases, operators must ask how much permeability gain is sufficient, and what level of seismicity is too much. At the same time, if induced seismicity is to be used as a monitoring tool (for instance, to map the stimulated volume of rock during an EGS project), operators need to know what 
information it provides. If the occurrence of seismicity implies a perturbation of porefluid pressure or reservoir stress change, does it also imply change in permeability? In order to satisfactorily answer these questions, the relationship between seismicity and reservoir permeability must be better understood.

The seismicity-permeability relationship has been widely studied in laboratory settings for a number of years (e.g. Lee and Cho, 2002, and citations therein). Generally, when slip occurs on a preexisting fracture, the permeability of that fracture also increases through a process known as self-propping, where the misalignment of asperities on opposing fracture walls leads to an increase in fracture aperture, and thus permeability (Figure 1.16). The degree to which fracture permeability is affected by slip depends on the length of the slip vector, rupture velocity, the roughness of the fracture surface and the material within the fracture (gouge) (Fang et al. 2017). Fracture permeability can also increase as a result of displacement normal to the fracture plane through processes such as thermal contraction of the fracture walls or pore pressure increase (Figure 1.16).

Guglielmi et al. (2015) and Cappa et al. (2019) measured shear and normal displacement during injection into a decameter-scale fault and found that fault permeability was most sensitive to normal displacements early in the injection, with relatively little permeability gain related to shear displacements induced at later stages of the injection. This result questions the commonly-held belief that the stimulation (i.e. permeability increase) of injection wells at sub- $\sigma_{3}$ pressures, is due to induced shear on hydraulically-connected fractures. Instead, permeability increase could be largely due to normal displacements that are aseismic due to possible rate-strengthening behavior induced by fluid injection. In turn, this calls into question the use of seismicity as a way to map the extent of the stimulated reservoir (e.g. Fang et al., 2018; Yoon et al., 2014; Dempsey et al., 2015). This permeability-seismicity decoupling was recently illustrated through modeling of the Habanero and Paralana EGS project in Australia (Riffault et al., 2018).

Aseismic motions are important contributors to permeability change in isothermal environments, such as shallow mines (Guglielmi et al., 2015), or shale reservoirs (Das and Zoback, 2011). However, they may play an even more important role in hightemperature geothermal environments where thermoelastic contraction of fracture walls may serve to encourage fracture normal displacement. A model where a borehole's permeable zones expand and contract in response to thermal stress is widely accepted at geothermal fields in New Zealand (Grant et al., 2013), where it has been used to describe oscillating injectivity behavior in response to injection-related cooling, and subsequent heating in the absence of injection.

A final, and more difficult to characterize, factor influencing reservoir permeability is fluid chemistry. As fluid moves through a formation, it may dissolve minerals out of, or precipitate minerals into, fractures, depending on the pressure and temperature 
and composition of the fluid. Though less well-understood, this effect probably plays a large part in creating or destroying permeability through opening or sealing of fractures that would be likely to fail (Clearwater et al. 2015). In this work, we do not address this issue, but it must be noted as a source of uncertainty with respect to reservoir permeability.

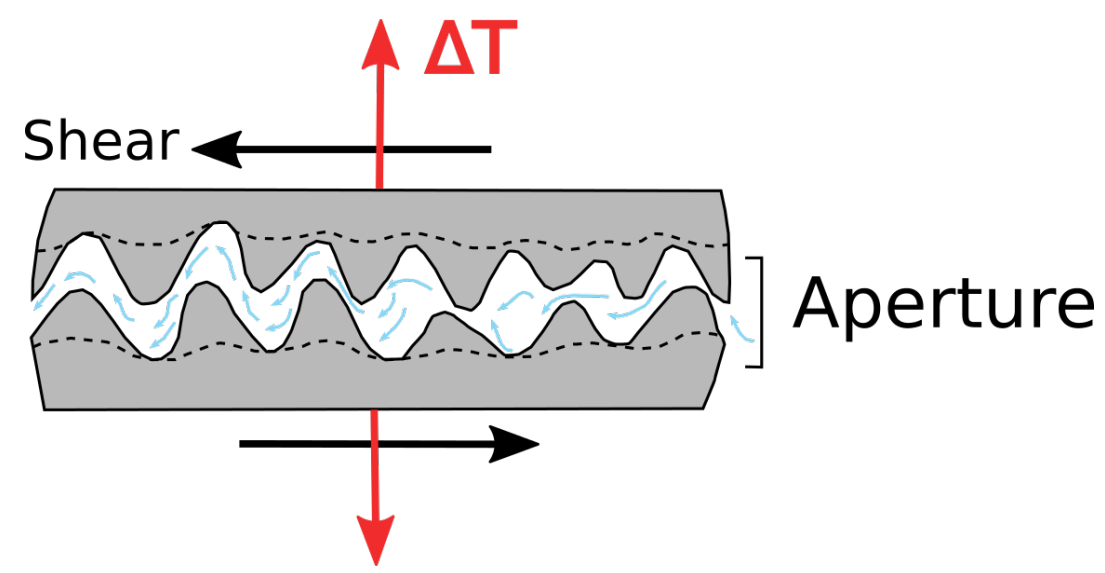

Figure 1.16: Simple schematic, adapted from Fang et al. (2017), illustrating the possible fracture aperture increase associated with slip on a fault, fracture opening (due to pressure increase or thermal contraction of the fracture walls), or some combination of both.

\subsection{Study aims}

The primary goal of this thesis is to establish quantifiable relationships between the occurrence of seismicity and power production operations, specifically fluid extraction and injection, at the Rotokawa and Ngatamariki geothermal fields. The specific research questions that we address are as follows:

1. Are variations in the rate, location and magnitude of seismicity at each field correlated with rates of injection and withdrawal of fluid?

2. Does well stimulation (i.e. permeability increase) correspond to periods of increased seismicity?

3. Do earthquake focal mechanism solutions reflect the orientation and extent of flowing fractures?

4. Do spatial and temporal variations in stress state correspond to injection and production changes in the reservoirs?

In order to answer these questions, we construct a comprehensive catalog of seismicity for both geothermal fields. This process will include the completion of the following tasks: 


\begin{tabular}{cccc} 
Field & Operation & Start & End \\
\hline Ngatamariki & NM08 stimulation & $2012-6$ & $2012-7$ \\
Ngatamariki & NM09 stimulation & $2012-12$ & $2013-1$ \\
Ngatamariki & NM10 drilling losses & $2012-7$ & $2012-8$ \\
Ngatamariki & NM12 drilling losses & $2014-6$ & $2014-9$ \\
Rotokawa & RK24 injectivity decline & 2012 & 2013 \\
Rotokawa & Injection switch: RK24-RK23 & $2014-6$ & $2014-7$ \\
Rotokawa & RK34 drilling losses & $2014-9$ & $2014-11$ \\
Rotokawa & Plant maintenance & $2012-2015$ & $2012-2015$
\end{tabular}

Table 1.1: Table summarizing the key periods of interest specified by Mercury for the years 2012-2015. These periods define the focus of study for comparing changes in well parameters to the response in seismicity.

- Compile an automatically-detected catalog of seismicity (Question 1, Chapter 2);

- Detect additional, smaller-magnitude events using the automatically-detected events as templates in a matched-filter routine (Chamberlain et al., 2017a, Questions 1-2, Chapters 2-4);

- Relocate the entire catalog using a non-linear, grid search algorithm Lomax et al., 2014), with locations further refined by a relative-relocation algorithm (Trugman and Shearer, 2017, Questions 1-2, Chapters 2-5);

- Implement a magnitude estimation methodology (Shelly et al., 2016a) for events detected using the matched-filter technique (Questions 1-2, Chapters 2-4);

- Manually pick P-wave first arrival polarities and invert for focal mechanism solutions (Walsh et al., 2009, Questions 3-4, Chapter 5);

The analysis of this catalog as it relates to the injection and production operations at the fields will involve the following additional step:

- Invert focal mechanism solutions for stress state over time and space Arnold and Townend, 2007; Lund and Townend, 2007, Question 4, Chapter 5);

The research presented here was funded by Mercury NZ Ltd., who have identified several periods of interest in which the reservoir behaved in unexpected or interesting ways. We will focus on addressing each of these periods in turn and relate the characteristics of seismicity within the reservoir to the parameters measured at the wells in question. The periods of interest are summarized in Table 1.1 .

\subsection{Thesis structure}

Following this introductory chapter, Chapter 2 discusses, in detail, the seismic data and network that provide the basis for this work. It also addresses the supplemental, 
borehole data gathered by Mercury and describes the basic methods used to detect, locate, determine magnitudes and calculate focal mechanisms for seismic events.

Chapters 3, 4 and 5 contain distinct, standalone projects and have been written and formatted with the intent of publication as peer-reviewed papers.

Chapter 3 is published in G-Cubed as a manuscript entitled, "Seismic response to injection well stimulation in a high-temperature, high-permeability reservoir" and addresses the relationship between seismicity and injection during the stimulation and completion tests undertaken prior to the startup of the Ngatamariki power plant Hopp et al., 2019). Coauthor Stefan Mroczek contributed automatic S-picks to the catalog presented in this and all other chapters. A more detailed methodology for this process is described in Mroczek et al. (2019) and Mroczek (2017). In addition, coauthor Steven Sewell contributed geologic context and interpretation of these results.

Chapter 4 has a similar structure to Chapter 3 but pertains to Rotokawa. It relates the rate, location and magnitude of seismicity to changes in injection in 2012-2015. It also attempts to establish relationships between the frequency-magnitude distribution of Rotokawa seismicity and the reservoir pressure distribution.

Chapter 5 details the spatial and temporal variations in focal mechanisms, magnitudes and stress at both Ngatamariki and Rotokawa between 2012 and 2015.

Finally, Chapter 6 summarizes the findings of the preceding three chapters and suggests potential paths along which the research presented here could continue.

Chapters 3, 4 and 5 each contain detailed descriptions of the methodologies used. Therefore, Chapter 2 only addresses the methods used in creating the underlying earthquake catalog, which includes linearized, grid-search and double-difference earthquake locations, event magnitudes and event focal mechanisms. All further explanation of the more complex methods applied in the standalone chapters will therefore be addressed in the appropriate chapter.

The vast majority of the work presented in this thesis was conducted by me (Chet Hopp). However, I have chosen to write this thesis in the first-person plural, as is consistent with, and in acknowledgment of, the significant contributions of both my supervisors and colleagues, who have been included in Chapter 3 as authors of the submitted manuscript and will contribute to forthcoming manuscripts. To this point, the initial, amplitude-based detection and linear location of the earthquake catalog from which this work began was conducted by a team of researchers at GNS Science in Wairakei and elsewhere, under contract to Mercury. That notable contribution was important in directing the rest of this research. 


\section{Data and methods}

This thesis relies on the analysis of an earthquake catalog which, along with raw waveform data, is the basis of seismology. This chapter describes a number of methods used to derive a catalog of seismicity from raw, unfiltered waveforms recorded at seismograph stations. At its most basic, our earthquake catalog for Rotokawa and Ngatamariki contains information about the time of occurrence, location and magnitude of the event and, in some cases, information about how the fault or fracture slipped (focal mechanism). What follows is a description of the methods of detection, location, magnitude estimation and focal mechanism determination employed in building the catalog, as well as a description of the independent datasets used to contextualize our findings.

\subsection{Data}

\subsubsection{Seismic network}

The seismic data presented in this thesis were recorded on a network of between 15 and 29 seismometers, most of which are concentrated in an area $15 \mathrm{~km} \mathrm{~N}-\mathrm{S}$ by $7 \mathrm{~km} \mathrm{E}-\mathrm{W}$ centered around the Rotokawa and Ngatamariki geothermal fields (Figures 2.1 and 2.2). The vast majority of the stations are owned and operated by Mercury NZ Ltd., for the purpose of monitoring the geothermal fields. However, six stations operated by either Contact Energy (ARAZ, THQ2) or GeoNet, the national seismic network (HRRZ, PRRZ, ALRZ, WPRZ), have been incorporated into this network to improve station coverage.

GNS Science in Wairakei has been contracted to maintain the seismic network at the geothermal fields; a task that includes quarterly retrieval of data, which are stored on-site at each station between servicing trips. As detailed in Table 2.1, the Mercuryoperated stations are $4.5 \mathrm{~Hz}$ geophones and the data are sampled at $200 \mathrm{~Hz}$. Three of these stations are borehole instruments (NS12, NS13, NS14), deployed in the center of the Ngatamariki field at depths of between 202 and $514 \mathrm{~m}$. The GeoNet data are sampled at $100 \mathrm{~Hz}$ and were downloaded and archived locally at the outset of this project in order to speed up analysis.

As the Rotokawa reservoir has been developed for considerably longer than Ngatamariki, its seismic network (stations named RT) has been in operation for over a decade. Ngatamariki stations began to be installed prior to drilling operations in mid- 
2012. Network coverage varied with time as certain stations were moved to improve performance or were replaced after damage due to livestock or vandalism (Figure 2.2). Therefore, the network presented in Figure 2.1 does not represent the exact geometry for any specific point in time, instead reflecting the locations of instruments that were operational at any point between 2012 and 2015.

\subsubsection{Preliminary earthquake catalog}

As stated above, the initial earthquake catalog was constructed by GNS Science using the software package SeisComP3 (Weber et al., 2007). Event detection was done using a short-term average/long-term average technique, which records a detection when the ratio of the average amplitude in a user-defined, short-term window to that in a long-term window exceeds a threshold value. Each of these detections was then automatically located using the linearized location algorithm Hypo 71 (Lee and Lahr. 1972). See Sections 2.2.1 and 2.2.3 for a detailed description of these techniques.

Local magnitudes $\left(\mathrm{M}_{L}\right)$ were also calculated using the SeisComP3 module scmag, which uses the ubiquitous equation from Richter (1935) to calculate magnitude at an individual station:

$$
M_{L}=\log _{10} A-\log _{10} A 0
$$

where $A$ is the amplitude of the seismic trace in $\mathrm{mm}$ and $A 0$ is a calibration term that is used to adjust the scale to different localities. In this case, the SeisComP3 $\log A 0$ module was used with distance calibration values of $A 0$ summarized in Table 2.2, The magnitudes at each station were averaged to return the final event magnitude.

The national seismic network, GeoNet, reports location and magnitude information for most earthquakes larger than magnitude 2.1 in the area of Rotokawa and Ngatamariki (Sherburn et al., 2015). The GeoNet-calculated magnitudes of such events at the geothermal fields were compared with the independently-calculated magnitudes from the stations in the Mercury network used in this study (note that three stations from the GeoNet network were included in the Mercury seismic network). There was found to be a disagreement between GeoNet- and Mercury-calculated magnitudes of -0.32 magnitude units, which was addressed by applying a static correction of +0.32 to all Mercury-network magnitudes in the preliminary catalog used in this thesis. The corrected magnitudes are compared to the GeoNet magnitudes in Figure 2.3, showing that the relationship between the two sets of magnitudes is approximately 1:1.

Following the steps outlined above, GNS Science provided us the RotokawaNgatamariki earthquake catalog in the form of SeisComP3 native sc3ml xml files. This catalog contained 8226 earthquakes that occurred between 15 May, 2012 and 18 November, 2015 (black events, Figure 2.4). These two dates correspond to the date of the start of the first Ngatamariki seismographs, at which time the Rotokawa and 


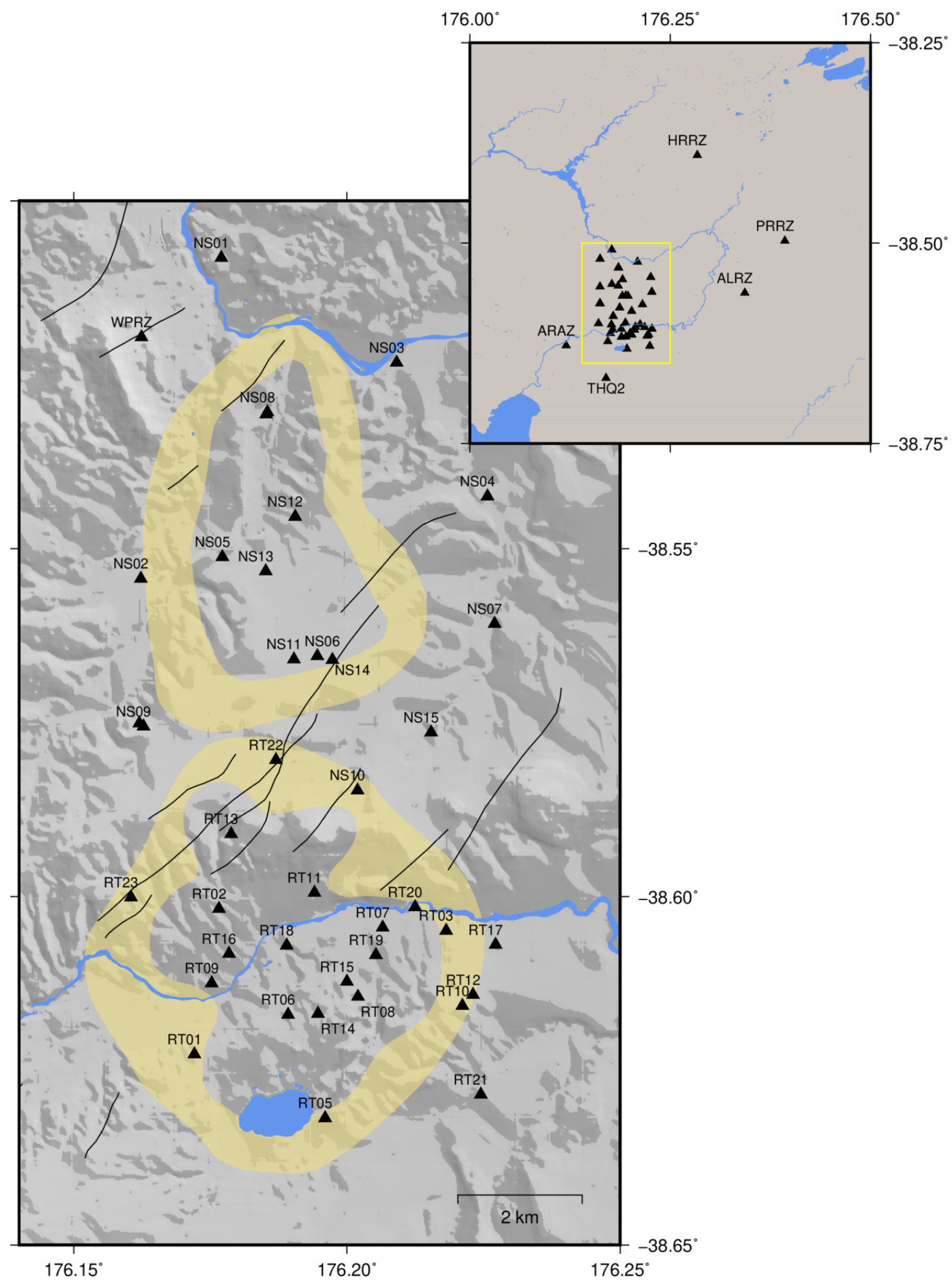

Figure 2.1: All stations that were active at any time between 2012 and 2015. Goldenrod shapes indicate the most likely resistivity boundaries for the Ngatamariki and Rotokawa geothermal fields, triangles show the locations of seismic stations, each of which is labeled with the station name appearing in Table 1, below. 


\begin{tabular}{|c|c|c|c|c|c|}
\hline Station name & Longitude $\left({ }^{\circ}\right)$ & Latitude $\left({ }^{\circ}\right)$ & Elevation (m) & Depth $(\mathrm{m})$ & Sensor \\
\hline ALRZ & 176.343014 & -38.562043 & 405 & 0 & L4C-3D \\
\hline ARAZ & 176.12006 & -38.62769 & 420 & 0 & L4C-3D \\
\hline HRRZ & 176.283754 & -38.39013 & 590 & 0 & L4C-3D \\
\hline NS01 & 176.177 & -38.5082 & 349 & 0 & Geospace GS-11D \\
\hline NS02 & 176.162275 & -38.554318 & 375 & 0 & Geospace GS-11D \\
\hline NS03 & 176.209102 & -38.523259 & 327 & 0 & Geospace GS-11D \\
\hline NS04 & 176.225718 & -38.542487 & 345 & 0 & Geospace GS-11D \\
\hline NS05 & 176.1772 & -38.5512 & 386 & 0 & Geospace GS-11D \\
\hline NS06 & 176.194571 & -38.565449 & 407 & 0 & Geospace GS-11D \\
\hline NS07 & 176.227019 & -38.560793 & 345 & 0 & Geospace GS-11D \\
\hline NS08 & 176.185438 & -38.530393 & 345 & 0 & Geospace GS-11D \\
\hline NS09 & 176.162759 & -38.575539 & 451 & 0 & Geospace GS-11D \\
\hline NS10 & 176.201928 & -38.584723 & 400 & 0 & Geospace GS-11D \\
\hline NS11 & 176.1903 & -38.5659 & 409 & 0 & Geospace GS-11D \\
\hline NS12 & 176.190524 & -38.545408 & 350 & 514 & IESE Borehole: Duke 4.5 \\
\hline NS13 & 176.185137 & -38.553235 & 380 & 250 & IESE Borehole: Duke 4.5 \\
\hline NS14 & 176.197332 & -38.565986 & 365 & 202 & IESE Borehole: Duke 4.5 \\
\hline NS15 & 176.2154 & -38.5764 & 382 & 0 & Geospace GS-11D \\
\hline NS16 & 176.162 & -38.5751 & 450 & 0 & Geospace GS-11D \\
\hline NS18 & 176.1852 & -38.5307 & 348 & 0 & Geospace GS-11D \\
\hline PRRZ & 176.393 & -38.4971 & 392 & 0 & $\mathrm{~L} 4 \mathrm{C}-3 \mathrm{D}$ \\
\hline RT01 & 176.17208 & -38.62264 & 371 & 0 & LE-3Dlite \\
\hline RT02 & 176.17658 & -38.60173 & 346 & 0 & LE-3Dlite \\
\hline RT03 & 176.21815 & -38.60488 & 337 & 0 & LE-3Dlite \\
\hline RT05 & 176.19599 & -38.63178 & 369 & 0 & LE-3Dlite \\
\hline RT06 & 176.18922 & -38.61694 & 366 & 0 & LE-3Dlite \\
\hline RT07 & 176.20653 & -38.60443 & 320 & 0 & LE-3Dlite \\
\hline RT08 & 176.20201 & -38.61436 & 341 & 0 & LE-3Dlite \\
\hline RT09 & 176.17524 & -38.61243 & 339 & 0 & Geospace GS-11D \\
\hline RT10 & 176.22112 & -38.61567 & 408 & 0 & Geospace GS-11D \\
\hline RT11 & 176.194057 & -38.599442 & 330 & 0 & Geospace GS-11D \\
\hline RT12 & 176.223066 & -38.614089 & 400 & 0 & Geospace GS-11D \\
\hline RT13 & 176.1788 & -38.591 & 428 & 0 & Geospace GS-11D \\
\hline RT14 & 176.1947 & -38.6168 & 380 & 0 & Geospace GS-11D \\
\hline RT15 & 176.200 & -38.6122 & 352 & 0 & Geospace GS-11D \\
\hline RT16 & 176.1784 & -38.6082 & 336 & 0 & Geospace GS-11D \\
\hline RT17 & 176.2272 & -38.6069 & 342 & 0 & Geospace GS-11D \\
\hline RT18 & 176.188968 & -38.606998 & 308 & 0 & Geospace GS-11D \\
\hline RT19 & 176.205294 & -38.608375 & 312 & 0 & Geospace GS-11D \\
\hline RT20 & 176.212467 & -38.601494 & 300 & 0 & Geospace GS-11D \\
\hline RT21 & 176.2245 & -38.6284 & 250 & 0 & Geospace GS-11D \\
\hline RT22 & 176.186985 & -38.580367 & 423 & 0 & Geospace GS-11D \\
\hline $\mathrm{RT} 23$ & 176.160466 & -38.600085 & 447 & 0 & Geospace GS-11D \\
\hline THQ2 & 176.1698 & -38.6684 & 450 & 80 & IESE Borehole: Duke 4.5 \\
\hline WPRZ & 176.1624 & -38.5196 & 519 & 0 & LE-3DliteMkII \\
\hline
\end{tabular}

Table 2.1: Summary of each station active at any time between 2012 and 2015 at Rotokawa and Ngatamariki. Most stations are owned and operated by Mercury NZ Ltd. However, stations ARAZ and THQ2 are owned and operated by Contact Energy and stations ALRZ, HRRZ, PRRZ and WPRZ are operated by the national seismic network, GeoNet. 


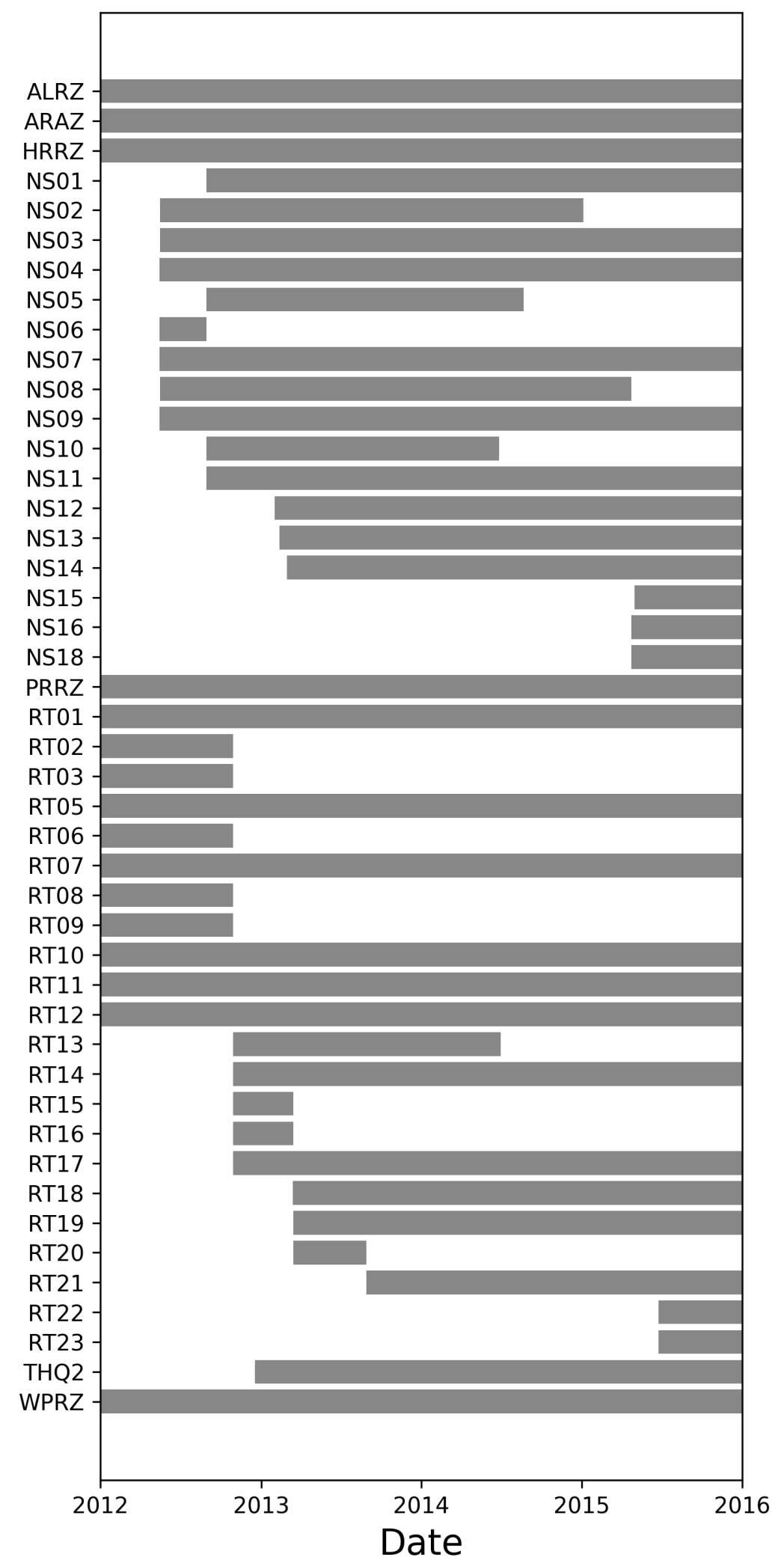

Figure 2.2: Data continuity plot for the seismic network detailed in Figure 2.1 and Table 2.1. Gray lines show the period during which each station was operational. 


\begin{tabular}{cc} 
Event-station dist $(\mathrm{km})$ & Calibration A0 (mag. units) \\
\hline 0 & 0.0431 \\
10 & -1.1563 \\
50 & -1.5755 \\
100 & -1.7560 \\
1000 & -2.3545
\end{tabular}

Table 2.2: Table summarizing the distance correction factors used by GNS Science in calculating the magnitudes of the preliminary earthquake catalog.

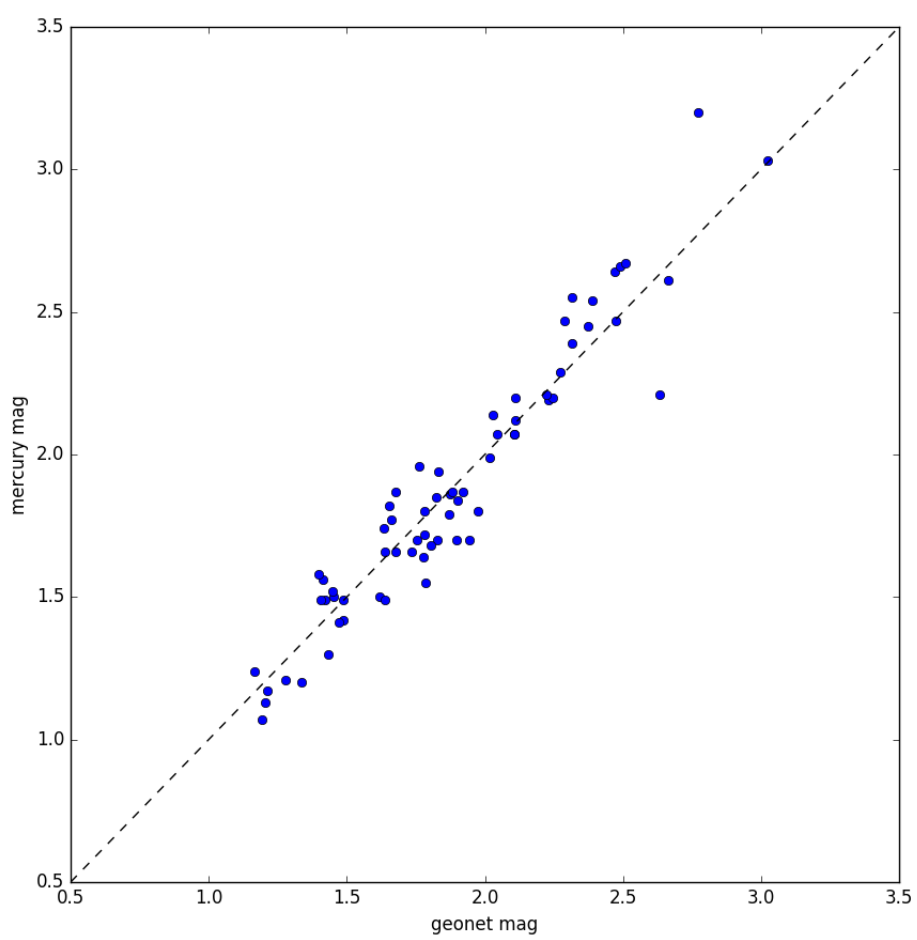

Figure 2.3: Mercury-calculated vs GeoNet-calculated magnitudes after applying a static correction of +0.32 magnitude units to the Mercury events.

Ngatamariki seismic networks were combined, and the final data-collection date of 2015, respectively. Many of these events occurred outside of the boundaries of the geothermal fields and were therefore marginally applicable to the aims of this thesis. For this reason, we filtered the catalog to include only events that occurred within or adjacent to the field boundaries of Rotokawa and Ngatamariki, and also to include events with average pick residuals within one standard deviation of the mean, leaving a total of 4405 events (Figure 2.4). These earthquakes were used as 'template' events in detecting additional events as detailed in Section 2.2.1 below. 


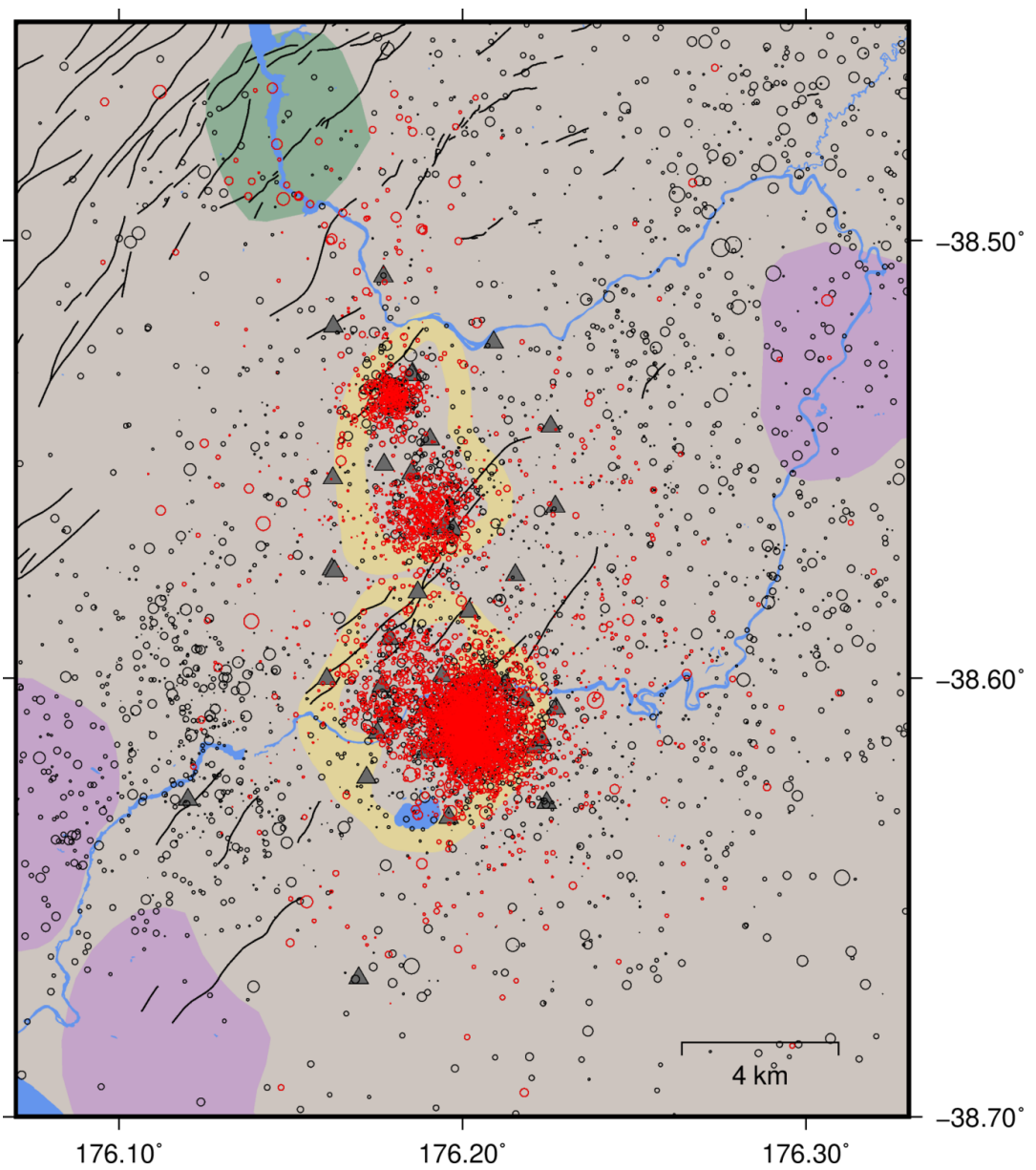

Figure 2.4: Overview of earthquakes in the preliminary GNS Science catalog. Red events are those which were selected for use in the analyses detailed in the following sections. Gray triangles represent seismograph stations. Yellow indicates the boundaries of the Ngatamariki and Rotokawa geothermal fields, purple nearby Contact Energyoperated fields, and green the protected Orakei-Korako geothermal field. Black lines show active faults (Langridge et al., 2016). Event symbols are scaled by magnitude where the largest is magnitude 4.2 .

\subsubsection{Mercury datasets}

In this thesis, we make use of a number of additional datasets that have been provided by Mercury. These data record the industrial processes taking place at the fields (such as injection and extraction of fluids) as well as properties of the deep reservoir that can be directly measured or inferred from the limited number of boreholes. They also provide important constraints for the interpretation of the earthquake catalog throughout this work.

\section{Injection and production rates}

The most important supporting datasets are pressure and flow rate time series for all injection and production wells. Specifically, they provide constraints on the reservoir 
pressures and injected volumes required to trigger or halt nearby seismicity. Flow and pressure time series are sampled daily during normal plant operations at both fields and more frequently during completion/stimulation tests at Ngatamariki (usually every five minutes). Pressure measurements can be taken either at the wellhead, in which case is they are referred to as wellhead pressure (WHP or at depth from within the wellbore, which is referred to as downhole pressure (DHP). Example flow rate and pressure data are shown in the introductory chapter.

\section{Pressure-temperature spinner data}

In practice, fluid does not exit (injection) or enter (production) a well uniformly across its entire length. Differences in permeability, controlled by rock type and fractures/ faults intersecting the borehole, mean that a well is hydraulically connected to the reservoir only at specific zones. These intervals are referred to as feedzones or permeable zones. In certain cases, operators will install a slotted or perforated liner in the well to dictate specifically where fluid will be able to move into or out of the well, however it is often common in geothermal reservoirs to leave the hole 'open' below the casing shoe. Even in the case of a perforated liner, the locations of the principal feedzones are unknown a priori. In order to infer feedzone locations, a tool called a

pressure-temperature spinner (PTS, essentially an impeller) is used, which measures flow, pressure and temperature within a well during injection. PTS tests are often run as a part of the completion testing of a well (Figure 2.5.

For a given flow rate, a strong variation in temperature over some depth interval typically implies that fluid is entering or leaving the well and that the corresponding depth may be a feedzone. In the case of the injection tests shown in Figure 2.5, strong 'heating' signals with depth signify that a relatively large volume of cold injectate is moving from the well into the reservoir, leaving hotter, conductively-heated fluid in the wellbore at greater depths. This information constrains the points from which fluid flows, and therefore helps us to interpret where pressure signals originate and how they relate to the characteristics of seismicity.

\section{Image logs}

In the case of many geothermal reservoirs, including Ngatamariki and Rotokawa, permeability is controlled by the reservoir fracture network. This is because the matrix rock is far less permeable than the typically extensive open fractures Grant and Bixley. 2011; Cant et al., 2018). A final important constraint on the locations of well feedzones, as well at the distribution and orientation of fractures over the entire reservoir, is resistivity- and sonic-based image logs. These logs are capable of showing detailed structures that intersect the wellbore including open (permeable) and sealed (impermeable) fractures and help corroborate data obtained from PTS measurements as to 


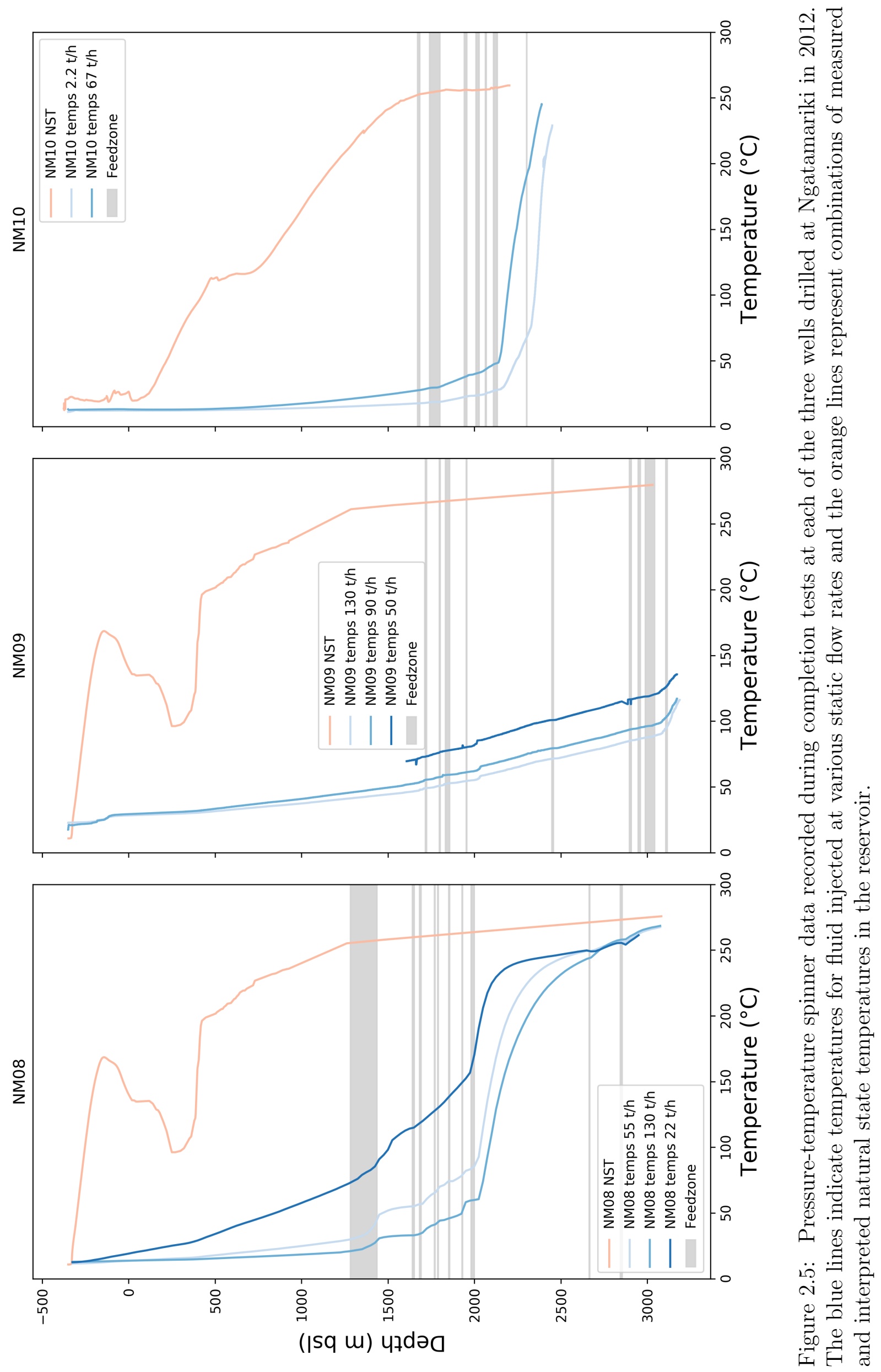


the location of feedzones (Figure 2.6). These measurements of reservoir fracture orientations are also useful constraints on the structures available to slip, and therefore help to constrain fault plane ambiguity in focal mechanism results.

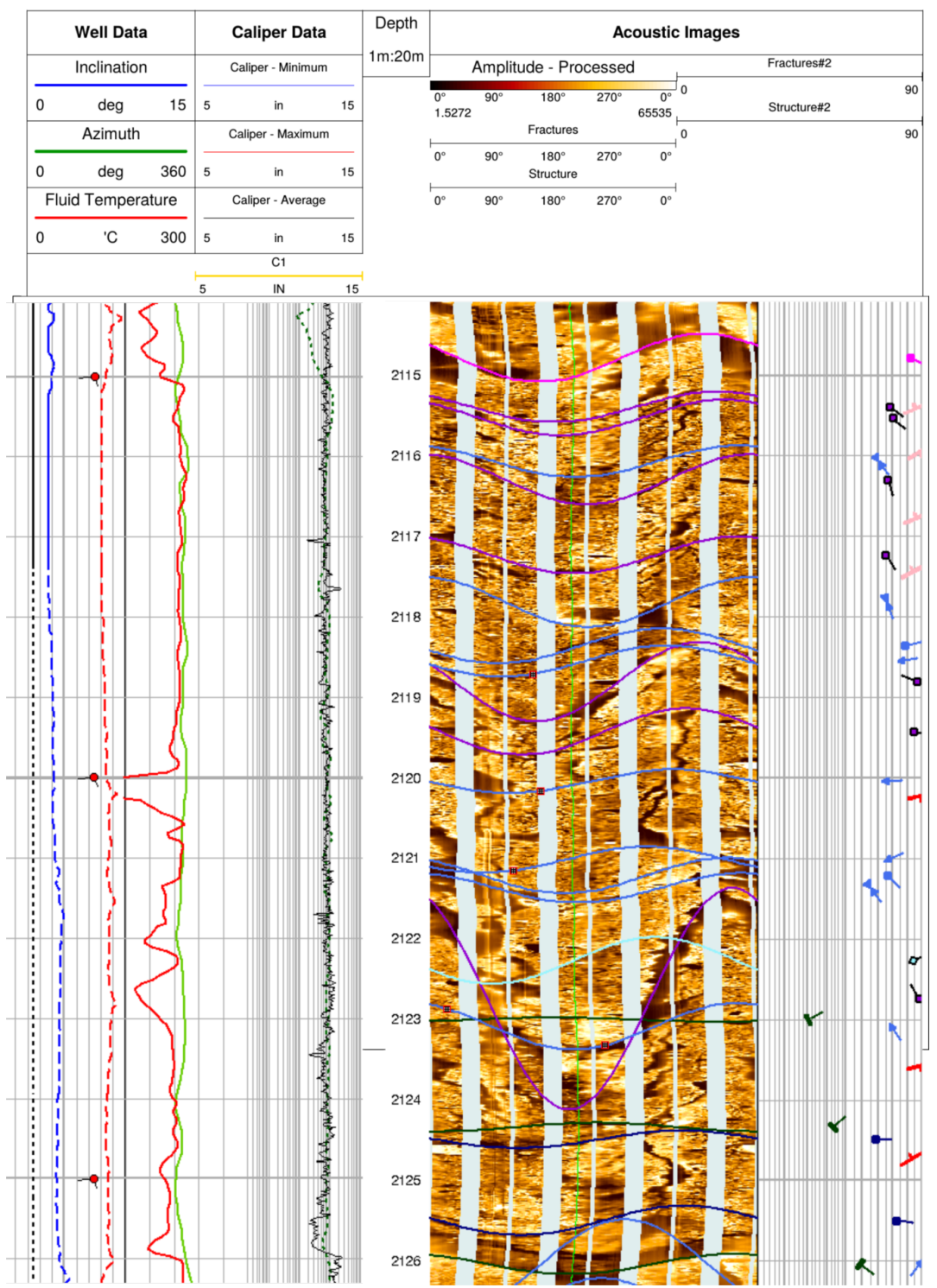

Figure 2.6: Example interval from the FMI image of Ngatamariki well NM10, showing interpreted fractures as well as other borehole data collected during the survey Halwa. 2013b).

\subsection{Methods}

\subsubsection{Earthquake detection}

In order to construct a catalog of induced seismicity, earthquakes must first be detected in a noisy stream of data. Detection is perhaps the most important task in the seismological workflow, yet the methods employed are imperfect and constantly being 
revised (Withers et al., 1998). In this thesis, we make use of two methods of detection, energy-based and correlation-based, the second built upon the first.

\section{Energy-based}

The energy released in an earthquake is proportional to the squared-amplitude of the signal recorded at a given seismograph station (Stein and Wysession, 2003). It is therefore intuitive that earthquakes can be detected by looking for changes in the amplitude of a single stream of seismic data, with the important caveat that the earthquake must have released a sufficient amount of energy to produce a signal of larger amplitude than the noise in the recorded data. The most ubiquitous technique for detecting earthquakes based on amplitude change is known as the STA/LTA method, whereby amplitudes in two overlapping windows of different duration are compared Withers et al. 1998). The ratio of the average amplitude of the short-term (STA) window to the long-term (LTA) window defines the detection statistic and a detection (sometimes referred to as a 'trigger') is recorded when the statistic exceeds a user-defined value.

A subsequent step in earthquake detection is known as 'association', which involves the grouping of triggers from multiple seismographs into distinct events. When only one or two triggers occur across a network, local noise sources may have triggered these stations, whereas the presence of many triggers across the network at a similar time likely signifies the occurrence of a larger phenomenon, perhaps an earthquake.

As mentioned above, the energy-based detection for this thesis was undertaken by GNS Science using the SeisComP3 software package. Triggering and station magnitude calculation was done using the module scautopick and event association using the module scautoloc, yielding the starting catalog for the correlation-based detection undertaken next.

\section{Correlation-based (matched filter)}

Where a stream of seismic data contains high levels of noise (e.g. in areas of extensive industrial activity) or a large number of signals, such as within an aftershock sequence, energy-based detection cannot realistically detect many of the events present in the data. Waveform cross-correlation has been used to overcome these deficiencies in finding known signals in such settings, and has been applied widely to problems including radar, digital communications and astronomy (e.g. Turin, 1960; Abbott et al., 2016). Correlating a known signal with a stream of data in search of a matching signal is often referred to as "matched-filter processing".

In seismology, matched filters have been adopted for use in finding a wide range of repeating and near-repeating signals that had eluded standard energy-based techniques, so long as the signal of interest was known. These applications include detection of local-to-regional scale crustal seismicity Schaff and Richards, 2011; Dodge and Walter. 
2015: Chamberlain et al., 2017a; Rawlinson, 2011), low-frequency and non-volcanic tremor at plate boundaries (Shelly et al., 2007; Chamberlain et al., 2014), volcanic and volcano-tectonic signals (Shelly et al. 2016a; Hotovec-Ellis et al., 2018), aftershock sequences (Warren-Smith et al., 2017) and explosions (Gibbons and Ringdal, 2006. 2012). In all instances, the known signal, be it an earthquake, radar pulse or nuclear explosion is referred to as the 'template' and the discovered, previously unknown signals are termed 'detections'. Throughout this thesis, we will also use the term 'family' to mean all detections generated by a single template. Detections in the same family are shown for template 2012sora451121 in Figures 2.7 and 2.8.
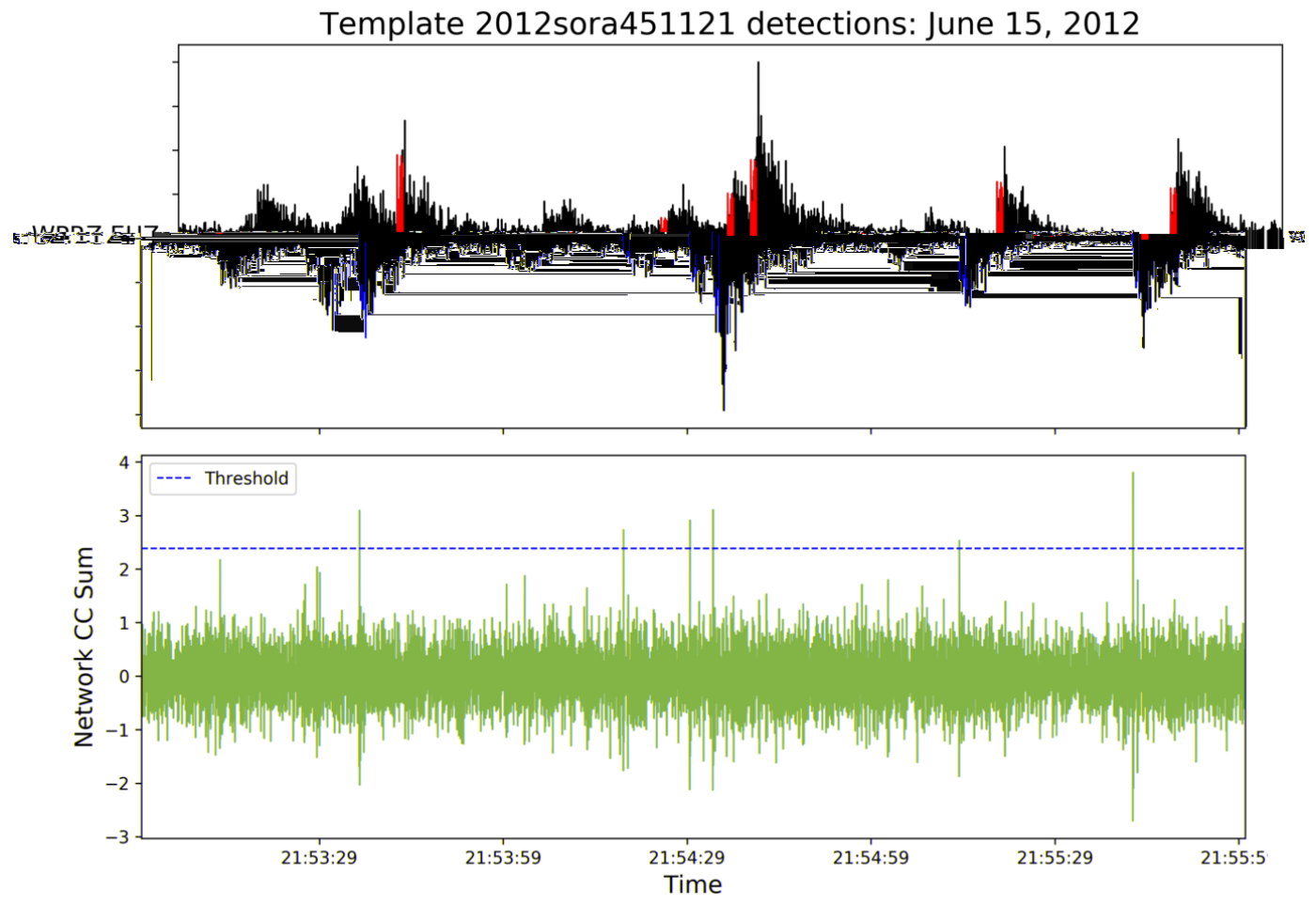

Figure 2.7: Detections shown only at station WPRZ for template 2012sora451121 on 15 June, 2012, during the stimulation of well NM08 (plot is three minutes long). The top panel shows template waveforms (red) overlain on continuous data (black). The bottom panel shows the cross-correlation sum for the entire network in blue with the daily detection threshold $(\mathrm{MAD} * 8)$ shown as a red dotted line.

In this thesis, we use the matched-filter method to detect repeating and nearrepeating, shallow ( $<10 \mathrm{~km}$ depth) crustal seismicity at the Rotokawa and Ngatamariki geothermal fields using the GNS Science catalog described above as template events. The cross correlation between a template event at a single station and the continuous seismic data being searched is described by the following equation (e.g. Chamberlain et al. 2017a): 

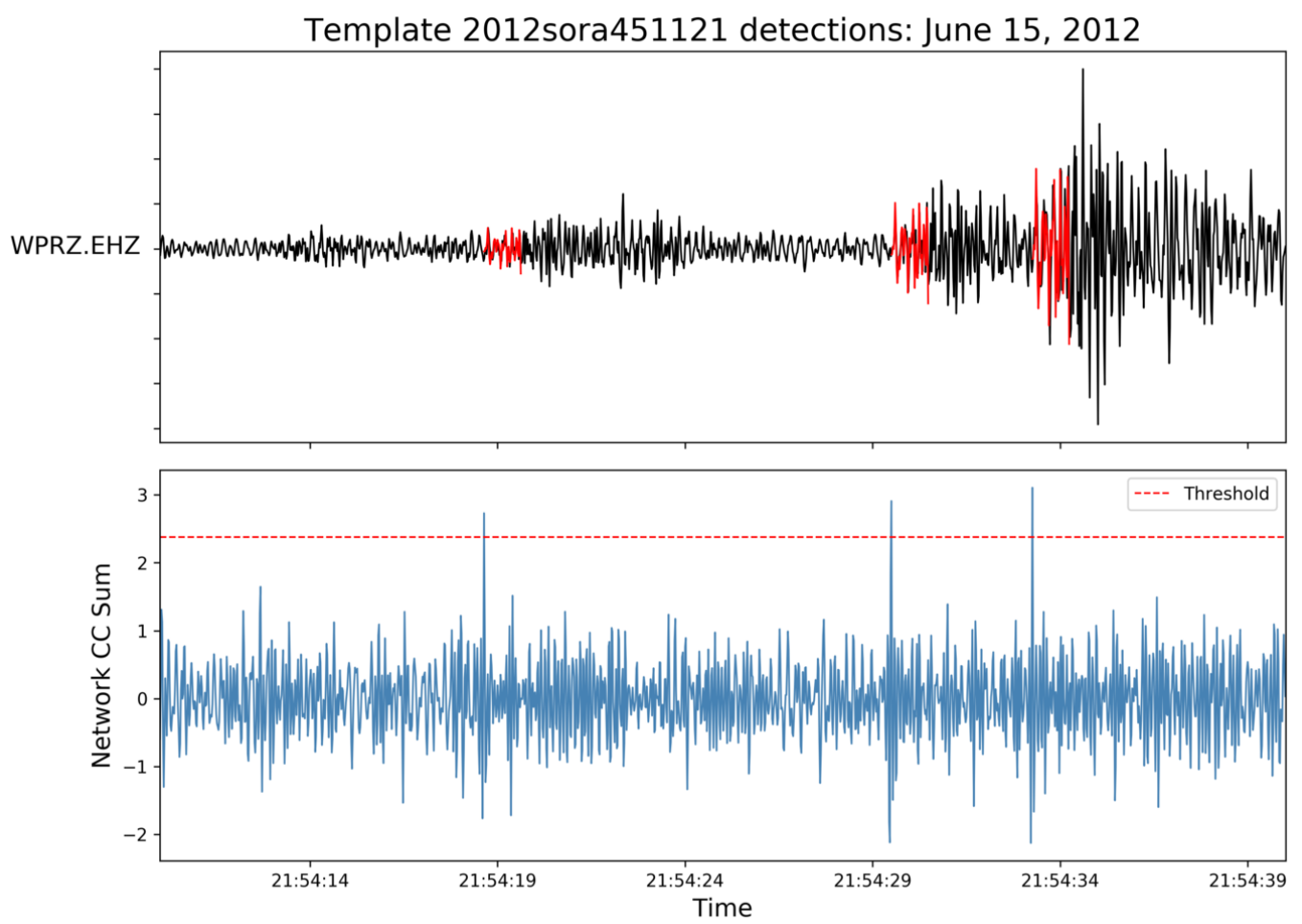

Figure 2.8: The same plot as above, zoomed-in to a 30-second interval that encompases three detections in order to show the waveforms in more detail.

$$
R(x)=\frac{\sum_{x^{\prime}=0}^{x+w_{x}}\left(T^{\prime}\left(x^{\prime}\right) \cdot I^{\prime}\left(x+x^{\prime}\right)\right)}{\sqrt{\sum_{x^{\prime}=0}^{x+w_{x}}\left(T^{\prime}\left(x^{\prime}\right)^{2} \cdot \sum_{x^{\prime}=0}^{x+w_{x}} I^{\prime}\left(x+x^{\prime}\right)^{2}\right.}}
$$

Here $I^{\prime}$ represents the continuous seismic data of interest and $T^{\prime}$ represents the template earthquake. $x$ represents the sample in the continuous data between sample 0 and sample $\left(N_{x}-w_{x}\right)$, where $N_{x}$ is the length of the continuous data being searched and $w_{x}$ is the length of the template. $x^{\prime}$ is the position within the window over which the correlation is being calculated.

This cross-correlation coefficient at each seismograph station is then summed to yield the network detection statistic (blue curves, Figures 2.7 and 2.8). The threshold for declaring a detection is calculated as the median absolute deviation (MAD) of the detection statistic for the entire period being searched (one day, for instance), multiplied by a user-defined value. In this thesis, we use a detection threshold of MAD * 8, as suggested by Shelly et al. 2007; Matson, 2019, red dotted line, Figures 2.7 and 2.8).

The template waveforms used in our matched-filter routine are one second long, starting 0.1 seconds before the P-pick, when present. During the energy-based detection, P-picks were only made on vertical channels. Therefore, templates consist of only vertical-channel waveforms (e.g. red waveforms in Figure 2.9, 2.7 and 2.8). Horizontal channels are not used due to the large uncertainties for the automatic S-picks in the 
GNS Science catalog. However, a length of one second ensures that both the P- and S-arrival are included in the template for each event due to the short travel times between events and stations at the geothermal fields (Figure 2.4). A length of one second also omits much of the coda, which is incoherent, even between highly-similar sources. This is especially apparent at Ngatamariki and Rotokawa, due to the highly-fractured reservoir, large variations in the volcanic geology (e.g. welded ignimbrites and ashfall deposits) and surface-deposit heterogeneity. After applying an anti-aliasing filter, waveforms were resampled at $50 \mathrm{~Hz}$ and filtered from 3.0 to $20.0 \mathrm{~Hz}$ to accommodate the high corner frequencies of events with event-station distances of $<15 \mathrm{~km}$. Continuous seismic data were processed in an identical manner to the templates.

\subsubsection{Arrival time picking for matched-filter detections}

\section{P-picks}

Cross-correlation between waveforms (Equation 2.2) is also commonly used as a means to increase the precision of arrival time picks for detected events and thereby improve the precision of hypocentral locations (e.g. Deichmann and Garcia-Fernandez, 1992, Aster and Rowe, 2000; Rowe et al., 2002; Stankova et al., 2008). Here we use a method similar to that described by Deichmann and Garcia-Fernandez (1992) whereby the cross correlation function (Figure 2.10b) between the template waveform (black trace, Figure 2.10 a) and detected waveform (red waveform, Figure 2.10a) is interpolated between the samples surrounding its maximum value. The maximum of the interpolated function is taken as the maximum cross-correlation value and the detection pick time is adjusted by the corresponding time lag (adjusted detection waveform; green, Figure 2.10 .

Using this method, the original P-picks for each of the newly detected events (initially recorded at the time of detection) were adjusted at each channel included in the template. For each station, template waveforms were correlated with the detected waveform over a 0.2 second window centered on the detection time. Picks were recorded at the time corresponding to the highest correlation value within that window. However, if the maximum correlation value of the template and detected waveform fell below 0.4 , the pick was discarded. We retained all events with more than five picks and discarded those with five or fewer. Figure 2.11 shows a series of detections (black) for template event 2013sora455868 at station RT01 after the P-picks were aligned. The stack of all detections is shown in blue at the bottom with a green line indicating the average pick time for all detections. Visually, the reader can see that the average pick time corresponds well to the time of the arrival of the P-wave in the stack, indicating that the newly adjusted-picks are consistent with the first arrival of energy (Rowe et al., $2002)$. 


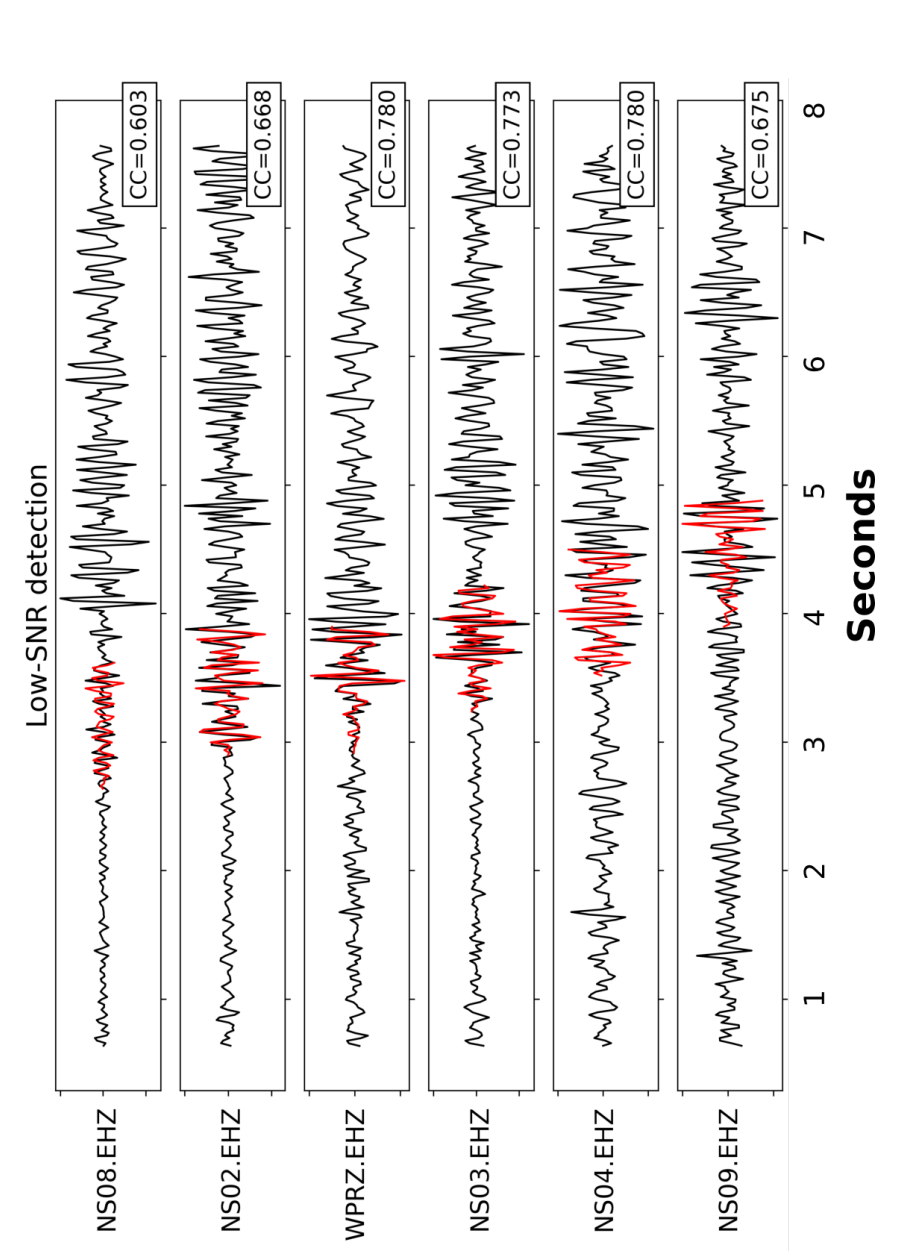

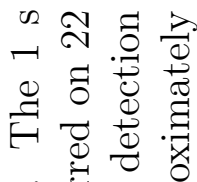

定芯氙芯

穵艺至

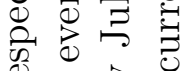

D 0

2 $\frac{\pi}{2}$

己ี

웡ำ

눈 0 过

.

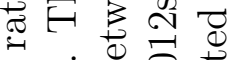

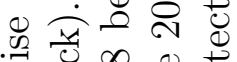

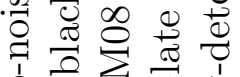

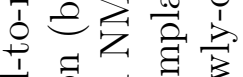

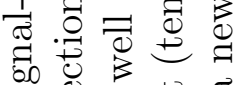

记吉

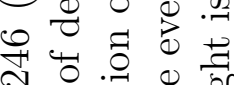

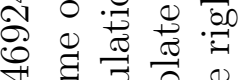

矛 寻

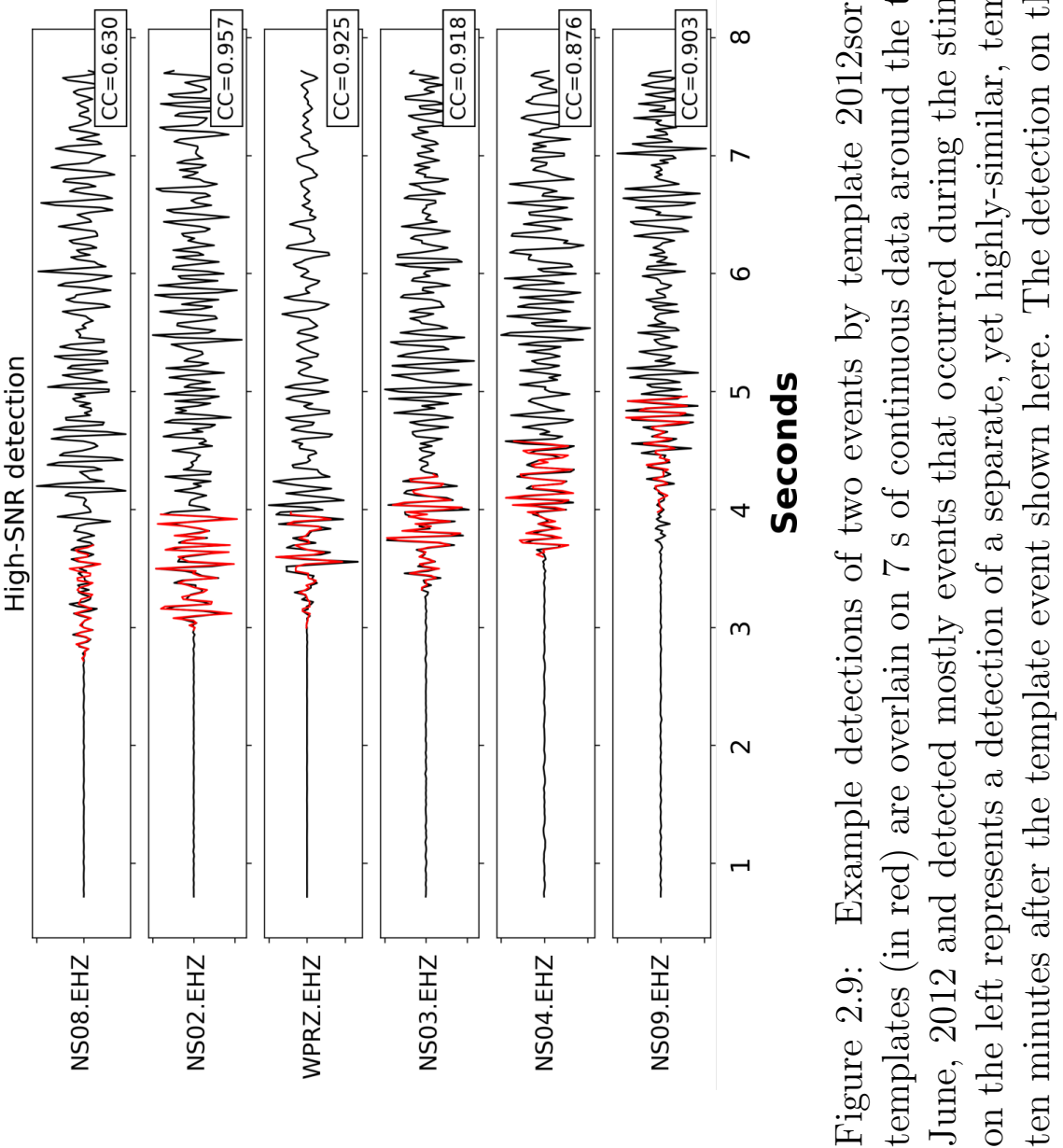



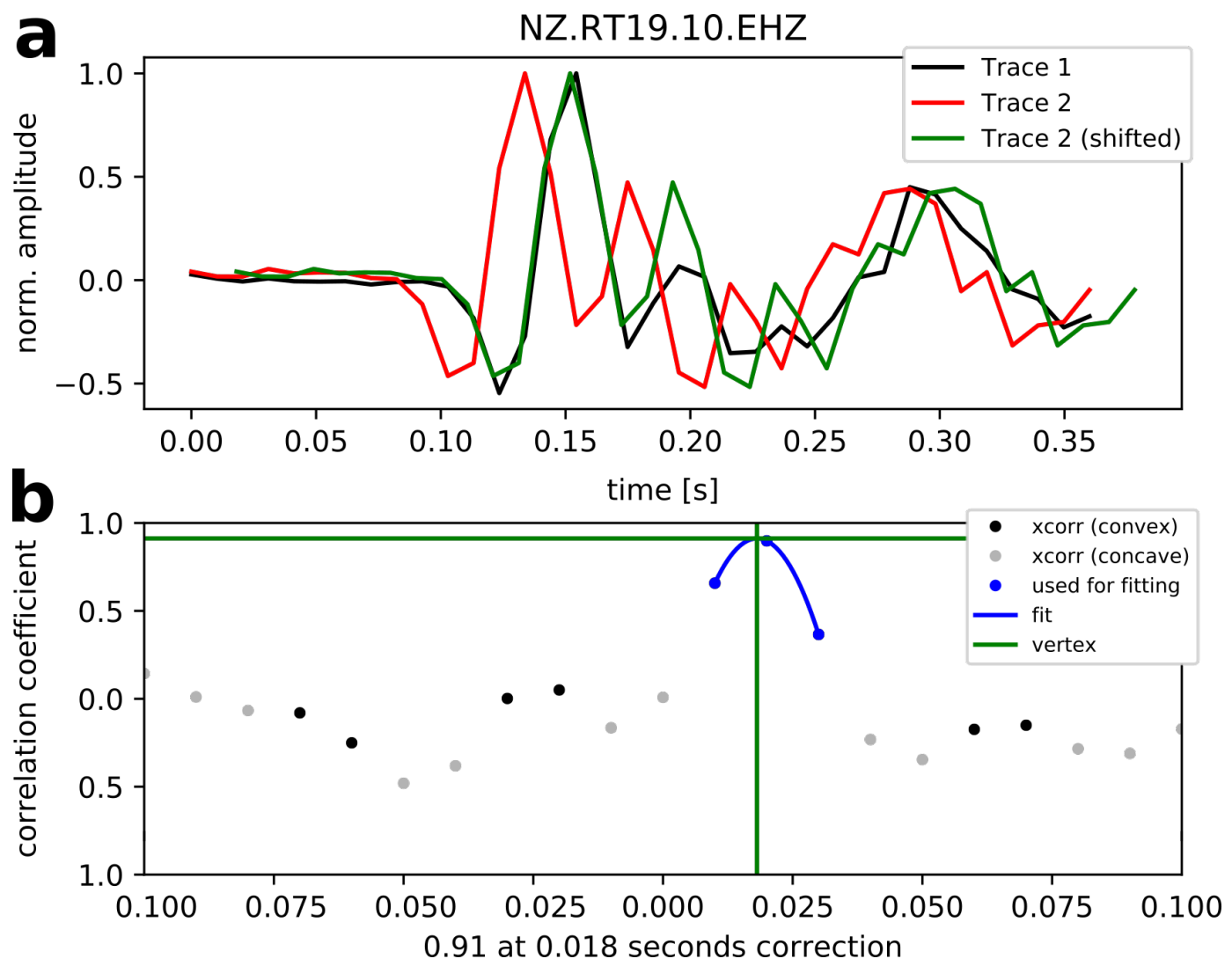

Figure 2.10: Example correlation-based pick correction for template 2014sora010105 and a single detection on the vertical channel of station RT19. a) shows the template (black), detection (red) and adjusted detection (green) waveforms. b) shows the crosscorrelation function (dots) for the two waveforms and a separate function fit to the samples surrounding the maximum (blue). The maximum of the blue function is then taken as the maximum correlation value, even though it occurs 0.007 seconds before the nearest sample in the cross-correlation function. For this example, the detection pick time is shifted earlier by 0.018 seconds. Plot created using the Obspy function xcorrpickcorrection() (The ObsPy Development Team, 2016).

\section{S-picks}

For events with six or more P-picks, MSc student Stefan Mroczek then made automatic S-picks using the method developed by Diehl et al. (2009) and modified by Castellazzi et al. (2015) called spickerC (Mroczek et al., 2019). The approach uses a weighted average of three different picking methods: STA/LTA (Allen, 1978), polarization detection (Cichowicz, 1993) and an autoregressive method based on the Akaike Information Criterion, developed by Leonard and Kennett (1999). The time uncertainties for each S-pick are calculated based on the spread of the three picks, with the uncertainty window defined by the earliest and latest pick time. Each pick is then assigned a quality class from 0-4 based on a user-defined classification scheme. (Mroczek et al., 2019) defined class 0 (the highest quality) as those picks having an error $\leq 0.3 \mathrm{~s}$ and a signalto-noise ratio of $>3$. We discarded all picks of class $>0$ and accepted only the S-phase 


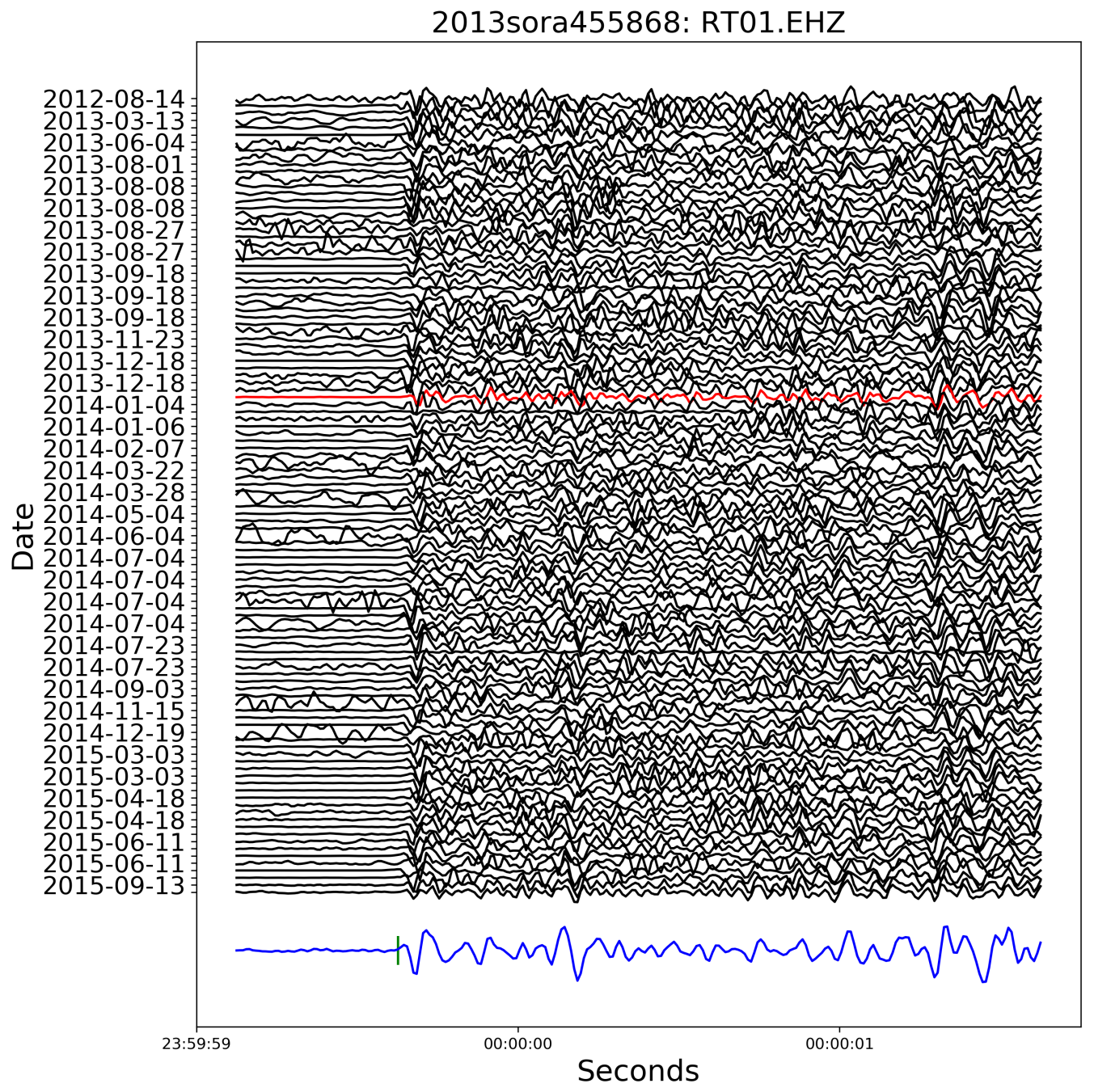

Figure 2.11: Detections for template 2013sora455868 after pick alignment via cross correlation. The template waveform is shown in red, while the detections are shown in black. Below, the normalized waveforms have been stacked (blue trace) and the mean pick time overlain on the stack in green, showing that the mean pick corresponds well to the arrival of the $\mathrm{P}$-wave.

pick at each station with the lowest error value. Example S-picks for a single station (North and East components) for each of the listed methods are shown in Figure 2.12. Further detailed description of the methodology and parameters used for the automatic S-arrival picking, can be found in Mroczek et al. (2019).

\subsubsection{Earthquake location}

Once a signal has been detected, the most critical piece of information regarding the event is its location (hypocenter). The locations contained in an earthquake catalog reveal subsurface features such as faults or the geometry of a subducting slab (for example), and spatio-temporal variations can reveal characteristics of dynamic trig- 


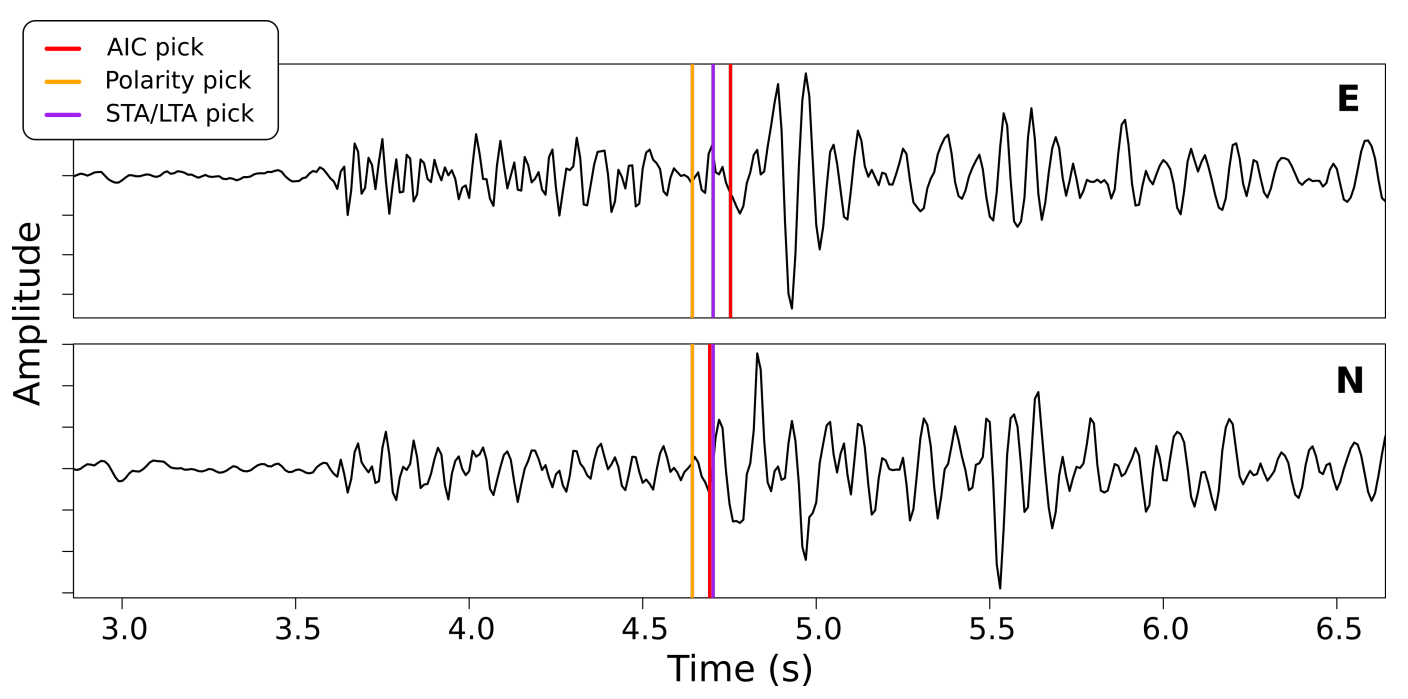

Figure 2.12: Example S-picks for the three methods employed by spickerC. Red corresponds to the AIC method of Leonard and Kennett (1999), yellow to the polarization method of Cichowicz (1993) and purple to the STA/LTA method described by (Allen. 1978). Figure modified from Mroczek (2017).

gering or fluid migration. The problem of determining an earthquake's location, in conjunction with its time of occurrence, can be forward-modeled from assumed source parameters or inverse-modeled from signal arrival times at a series of seismographs. The algorithms used to determine spatial location and origin time, collectively referred to solely as 'location' here, can be broken into three groups.

\section{Linear methods}

The most computationally efficient, and widely-used method for solving the non-linear location problem is to iteratively solve the forward problem posed in the following equation:

$$
t_{i}=T+\frac{1}{v} \sqrt{\left(X-x_{i}\right)^{2}+\left(Y-y_{i}\right)^{2}+\left(Z-z_{i}\right)^{2}}
$$

where $t_{i}$ is the arrival time of the ray traveling between the earthquake and recording station $i$, treating the earth as a homogeneous half-space with wave speed $v$. $T$ is the source time of the earthquake, $(X, Y, Z)$ are the longitude, latitude and elevation of the earthquake and $\left(x_{i}, y_{i}, z_{i}\right)$ are the longitude, latitude and elevation of station $i$.

Using the above equation, the arrival time at each station can be calculated by assuming a starting location, $(X, Y, Z)$, and origin time, $T$. The difference between the known arrival time at a given station, $t_{i}{ }^{\text {obs }}$, and the calculated arrival time, $t_{i}{ }^{\text {calc }}$, (often referred to as the 'misfit' or 'time residual', $r_{i}$ ) defines the quality of the chosen starting location: 


$$
r_{i}=t_{i}^{c a l c}-t_{i}^{o b s}
$$

Minimizing these residuals across a seismic network is the goal of all earthquake location approaches. The classical method used to solve the problem is an inverse approach, originally used by Geiger (1910), whereby a system of equations taking the form

$$
r_{i}=\frac{\partial t_{i}}{\partial X} \Delta X+\frac{\partial t_{i}}{\partial Y} \Delta Y+\frac{\partial t_{i}}{\partial Z} \Delta Z+\Delta t_{0}
$$

is solved iteratively until some condition, typically a threshold misfit value or location adjustment, is met. Here $\left(\Delta X, \Delta Y, \Delta Z, \Delta t_{0}\right)$ are the adjustments in the starting location parameters between iterations and the partial derivatives are expressed as follows:

$$
\begin{aligned}
\frac{\partial t_{i}}{\partial X} & =\frac{X-x_{i}}{v S} \\
\frac{\partial t_{i}}{\partial Y} & =\frac{Y-y_{i}}{v S} \\
\frac{\partial t_{i}}{\partial Z} & =\frac{Z-z_{i}}{v S}
\end{aligned}
$$

where $S$ represents the length of the source-receiver path. Geiger's original method solved the problem via a least-squares approach, although many other methods have been proposed and used since, including adding damping parameters and solving via singular-value decomposition (Thurber, 1985).

For many real-world scenarios, the above equations oversimplify the problem of earthquake location because they assume the velocity of the subsurface is homogeneous. For most locations where earthquakes occur, the substrate is heterogeneous, consisting of horizontal layers (as the simplest case) and growing in complexity to include strong lateral heterogeneities as well (faults, for example). This velocity heterogeneity is approximated by a velocity model, which expresses velocity changes with depth (1dimensional) and sometimes with geographic location as well (2- and 3-dimensional cases).

For this thesis, the original linearized locations are calculated in SeisComP3 via the algorithm of Lee and Lahr (1972), which uses a step-wise regression approach, and a velocity model calculated for the entire Taupō Volcanic Zone (Sherburn et al., 2003). For further relocations, we use a simple, 1-dimensional velocity model provided by Sewell et al. (2017) specifically for our seismic network (Table 2.3). 


\begin{tabular}{rc} 
Layer top $(\mathrm{km})$ & $V_{P}(\mathrm{~km} / \mathrm{s})$ \\
\hline-0.6 & 1.9 \\
0.2 & 2.6 \\
1.0 & 3.5 \\
1.5 & 3.6 \\
2.0 & 3.9 \\
3.0 & 4.9 \\
5.0 & 5.4 \\
10.0 & 5.81 \\
20.0 & 6.9 \\
50.0 & 7.43
\end{tabular}

Table 2.3: Local 1-D velocity model for Ngatamariki and Rotokawa from VELEST inversions

\section{Non-linear methods}

In cases where the velocity model is poorly known, velocity structure is highly-variable or earthquake locations are shallow or are outside of the seismic network, the linearization approaches described above may be insufficient to address a more highly-nonlinear location problem (Thurber, 1985). In this case, a number of additional approaches may be used. Thurber (1985), for example, suggested including the second order terms corresponding to the first-order Taylor series expansion described in Equations 2.6-2.8. These second-order terms can be especially important for shallow earthquakes located within a local seismic network and for short source-receiver paths. Foregoing linearization completely, Tarantola and Valette (1982) suggested a probabilistic approach whereby the posterior probability density function (pdf), $Q$, of the four unknown parameters (i.e. three spatial dimensions plus time) is described by (Lomax et al., 2014):

$$
Q(\mathbf{m})=k p(\mathbf{m}) \int_{\mathbf{D}} \frac{p(\mathbf{d}) F(\mathbf{d}, \mathbf{m})}{\mu(\mathbf{d}, \mathbf{m})} d \mathbf{d}
$$

where $k$ is a normalization constant, $\mathbf{m}$ is the vector of unknown hypocentral parameters, $\mathbf{d}$ is the vector of observed arrival-time data, $p(\mathbf{d})$ is the pdf describing the arrival-time uncertainties and $p(\mathbf{m})$ represents prior information about the hypocenter (e.g. from damage reports or known fault parameters). $F(\mathbf{d}, \mathbf{m})$ is the ability of the forward problem to describe the arrival times and $\mu(\mathbf{d}, \mathbf{m})$ is the homogeneous distribution over the arrival time data and hypocentral parameters (Lomax et al., 2014). The integral over the dataspace, $\mathbf{D}$, is typically referred to as the 'likelihood function', $L(\mathbf{x})$, and describes how well the given hypocenter represents the data.

For this thesis we have chosen to use the location program NonLinLoc Lomax et al. 2000), which makes use of Equation 2.9 and uses one of a number of grid-search algorithms to interrogate one of two possible likelihood functions, $L(\mathbf{x})$, one based on an L2-norm misfit function (Tarantola and Valette, 1982) and the other given by 
Equation 2.10. We have chosen to use this 'equal-differential time' likelihood function as it is more robust to outliers in the arrival time data (Lomax et al., 2014).

$$
L(\mathbf{x})=\left[\sum_{a, b} \frac{1}{\sigma_{a}^{2}+\sigma_{b}^{2}} \cdot \exp \left(-\frac{\left\{\left[T_{a}^{o}-T_{b}^{o}\right]-\left[T T_{a}^{c}(\mathbf{x})-T T_{b}^{c}(\mathbf{x})\right]\right\}^{2}}{\sigma_{a}^{2}+\sigma_{b}^{2}}\right)\right]^{N}
$$

Here $\mathbf{x}$ are the three spatial parameters of $\mathbf{m}$. The sum is taken over all possible $(N)$ observation pairs, $a$ and $b$, where $T_{a}^{o}$ and $T_{b}^{o}$ are the observed arrival times and $T T_{a}^{c}$ and $T T_{b}^{c}$ are the calculated travel times (arrival time minus the time of the event) for each observation, respectively. $\sigma_{a}$ and $\sigma_{b}$ represent the uncertainties of the observed arrivals times and calculated travel times (Lomax et al., 2014). The likelihood function maximizes where the difference in arrival times, $\left[T_{a}^{o}-T_{b}^{o}\right]$, and difference in calculated travel times, $\left[T T_{a}^{c}(\mathbf{x})-T T_{b}^{c}(\mathbf{x})\right]$, are equal, corresponding to the point in space, $\mathbf{x}$, that best represents observations $a$ and $b$. After summing over all possible pairs, the resulting probability density function has maxima at the spatial locations that satisfy the largest number of observation pairs, but does not attempt to fit all observations at once, making it more robust in the presence of arrival-time outliers. This formulation also eliminates the calculation of the event origin time, reducing the location problem from four to three dimensions (Lomax et al., 2000).

NonLinLoc first computes the travel times to all stations for each node in a userdefined grid. As the user may then attempt to solve the location problem using a number of gridding approaches, we selected the OctTree gridding method for all events at Rotokawa and Ngatamariki. The OctTree approach first calculates the probability that the source lies within any node in a coarse grid encompassing the entire, user-defined search area. This probability is proportional to the volume of the node and inversely proportional to the travel-time misfit calculated at its center (Lomax et al., 2000). The highest-probability node is then divided into eight equal-volume sub-nodes and the process is repeated, dividing the most likely of the sub-nodes into eight and continuing this process until a threshold is reached and the maximum-likelihood hypocenter found. Typically, the threshold for halting the program is a minimum node volume. NonLinLoc then samples the resulting OctTree structure of searched nodes in order to provide a representation of the location probability density function, which can then be used to characterize the uncertainty in the hypocenter solution (Lomax et al., 2000).

\section{Relative location methods}

Perhaps the most significant uncertainty inherent in the location problem is that associated with the subsurface velocity structure. In an attempt to minimize the effect of this uncertainty on the calculated locations, relative location methods were developed, initially as so-called 'master event' methods (e.g. Ito, 1985; Frémont and Malone, 
1987; VanDecar and Crosson, 1990), which determine the location of a cluster of events relative to a single, predefined 'master' event, provided each event's waveform is highlysimilar to that of the master. Got et al. (1994) expanded this approach to include larger catalogs by considering the delay times between all possible pairs of events instead of only events correlated with a predefined master. The Joint Hypocenter Determination (JHD) approach then inverted for hypocenters of a cluster of events as well as station and velocity model correction terms, so long as the cluster occupied a small enough volume that the slowness vector between each event in the cluster and any recording station could be assumed to be identical (Pujol, 1988; Dodge et al., 1996). These approaches were later expanded for use with even larger catalogs that occupied larger spatial footprints by considering an interconnected web of pairs of closely-spaced events. In this case, the slowness vector from each station to any event pair could be assumed to be identical while still relocating the event pair relative to more distant events. This approach is now commonly referred to as the double-difference method (Waldhauser and Ellsworth, 2000) and has been further expanded to include inversion for 3-dimensional velocity structure (Zhang and Thurber, 2003).

Specifically, instead of minimizing the misfit between observed and calculated arrival times for a single event, double-difference techniques seek to minimize the misfit between the observed and calculated arrival time (or travel time) differences between two paired events (hence, double-difference) given as (Waldhauser and Ellsworth, 2000)

$$
d r_{k}^{i j}=\frac{\partial t_{k}^{i j}}{\partial \mathbf{m}} \Delta \mathbf{m}^{i j}
$$

for phase $k$ and event pair $i, j$. Here the partial derivative $\frac{\partial t_{k}^{i j}}{\partial \mathbf{m}}$ represents the velocity vector between the centroid of event pair $i, j$ and the station that recorded phase pair $k . \Delta \mathbf{m}^{i j}$ is the vector of relative hypocentral shifts between $i$ and $j$ necessary to better fit the observed data.

All possible phases pairs, $k$, are combined into a system of linear equations $($ Waldhauser and Ellsworth, 2000)

\section{$\mathrm{WGm}=\mathrm{Wd}$}

where $\mathbf{G}$ is the $M \times 4 N$ matrix of partial derivatives ( $M$ double-difference observations and $N$ events), $\mathbf{W}$ is a weighting matrix, $\mathbf{d}$ is the matrix containing the doubledifferences, $d r_{k}^{i j}$, and $\mathbf{m}$ is the desired matrix of hypocentral shifts for each event in the catalog.

For large earthquake catalogs (e.g. $N>10,000$ and $M>1,000,000$ ), Equation 2.12 is often difficult to solve due to time and memory constraints. The most popular of the double-difference location programs, HypoDD (Waldhauser and Ellsworth, 2000), 
attempts to solve the system of equations, 2.12 by minimizing the L2-norm of the double-differences.

A recent, alternative approach to the double-difference problem is the GrowClust algorithm developed by Trugman and Shearer (2017), which we use for the final relocations of the catalogs presented in this thesis. The GrowClust algorithm makes use of the same data as HypoDD, namely travel-time differences between pairs of events (Equation 2.11), as well as absolute travel time data and starting locations. However, instead of attempting to solve the entire system of equations defined by every event pair (Equation 2.12), GrowClust creates a list of all event pairs in order of descending similarity (defined as the sum of the cross-correlation coefficients between their common waveforms). It then relocates each event pair relative to each other using a grid-search approach and minimizing the L1-norm of the residuals. Where an event pair joins two preexisting clusters, the relative locations of the pre-clustered events are held constant while the two clusters are relocated about their common centroid. This has the advantage of always retaining the relative relocations of higher-quality event pairs during subsequent relocation steps. This approach is also vastly more computationally efficient than the approach of HypoDD by avoiding solving Equation 2.12, thereby allowing GrowClust to include location error estimates via an iterative bootstrapping procedure that randomly resamples the input data and relocates the catalog. The location uncertainty is reported as the median absolute deviation of each event's location for each of the $n$ user-defined bootstrap iterations.

The application of these relative location techniques often proves imperative to obtaining precise locations, thus illuminating small-scale structures and migration of seismicity with time. Without these techniques, it can be difficult to interpret location results with any degree of confidence, especially at scales of $<10 \mathrm{~km}$, as at Rotokawa and Ngatamariki.

\subsubsection{Magnitude calculation}

The calculation of earthquake magnitude is typically done in conjunction with its detection and location and is the final, key parameter required for analysis of any earthquake catalog. There are a number of ways in which earthquake magnitude is calculated, usually based on the measured maximum amplitude of a signal recorded at a number of seismograph stations, but it can also be based on other measurements such as signal duration or full moment tensor inversion (Kanamori, 1983). In this thesis, magnitudes for the GNS-located template events are reported at local magnitudes $\left(\mathrm{M}_{L}\right)$, and discussed in detail in Section 2.1.2.

To compute the magnitude of the events detected by the matched filter, we use the method described by Shelly et al. (2016a), which uses pair-wise relative amplitudes 
between a template event and each of its detections to compute relative moments (Figure 2.13). This approach computes the relative amplitude, $\alpha$, as

$$
\alpha=v[2] / v[1]
$$

where $v[2]$ and $v[1]$ are the second and first elements, respectively, of the first row of the $2 \times 2$ matrix $V^{\prime}$ in

$$
M=U \Sigma V^{\prime}
$$

representing the singular value decomposition of a data matrix, $M$, with columns comprising both the template and the detected waveform on a single channel (Figure 2.13). The rows of $V^{\prime}$ are the right singular vectors, which map the weight of the left singular vectors, $U$, to the original data vectors and $\Sigma$ is a set of non-negative singular values. Because the template and detected waveforms in the data matrix are inherently similar, the first left singular vector, $U_{0}$, should describe only a difference in amplitude between template and detection. The relative amplitude between the two events can therefore be estimated as the ratio of the second and first elements, $v[2]$ and $v[1]$, of the first row of $V^{\prime}$.

We calculate relative amplitudes only when the cross-correlation coefficient between the template and detection exceeds 0.6 at any given station. Again, we note that template events only contain waveforms for the vertical channels. For those events recorded by a minimum of four stations exceeding the correlation threshold, we calculate the relative moment as the median of the relative amplitudes, following Shelly et al. (2016a). We note that, because the relative amplitudes are calculated from two waveforms recorded at the same station, there is no need to remove the instrument response.

This approach has proven to be more robust in the presence of relatively dissimilar waveforms than the method of Rubinstein and Ellsworth (2010), for example, which assumes high correlation coefficients between all pairs of events in a family (e.g. $\geq 0.85$ ) instead of simply between a template and each single detection, as assumed here. At Rotokawa and Ngatamariki, scattering and attenuation effects produce waveforms exhibiting lower degrees of similarity than typical repeating or near-repeating seismicity (e.g. along the San Andreas (Rubinstein and Ellsworth, 2010)).

We use the GNS Science $M_{L}$ (as described in Section 2.1.2) to calibrate the relative moment calculations from the method above and produce $M_{L}$ estimates for the matched-filter detections. This is done by first converting the template $M_{L}$ to $M_{w}$ using the scaling relationship (Ristau et al., 2016):

$$
M_{L}=0.97 M_{w}+0.14
$$



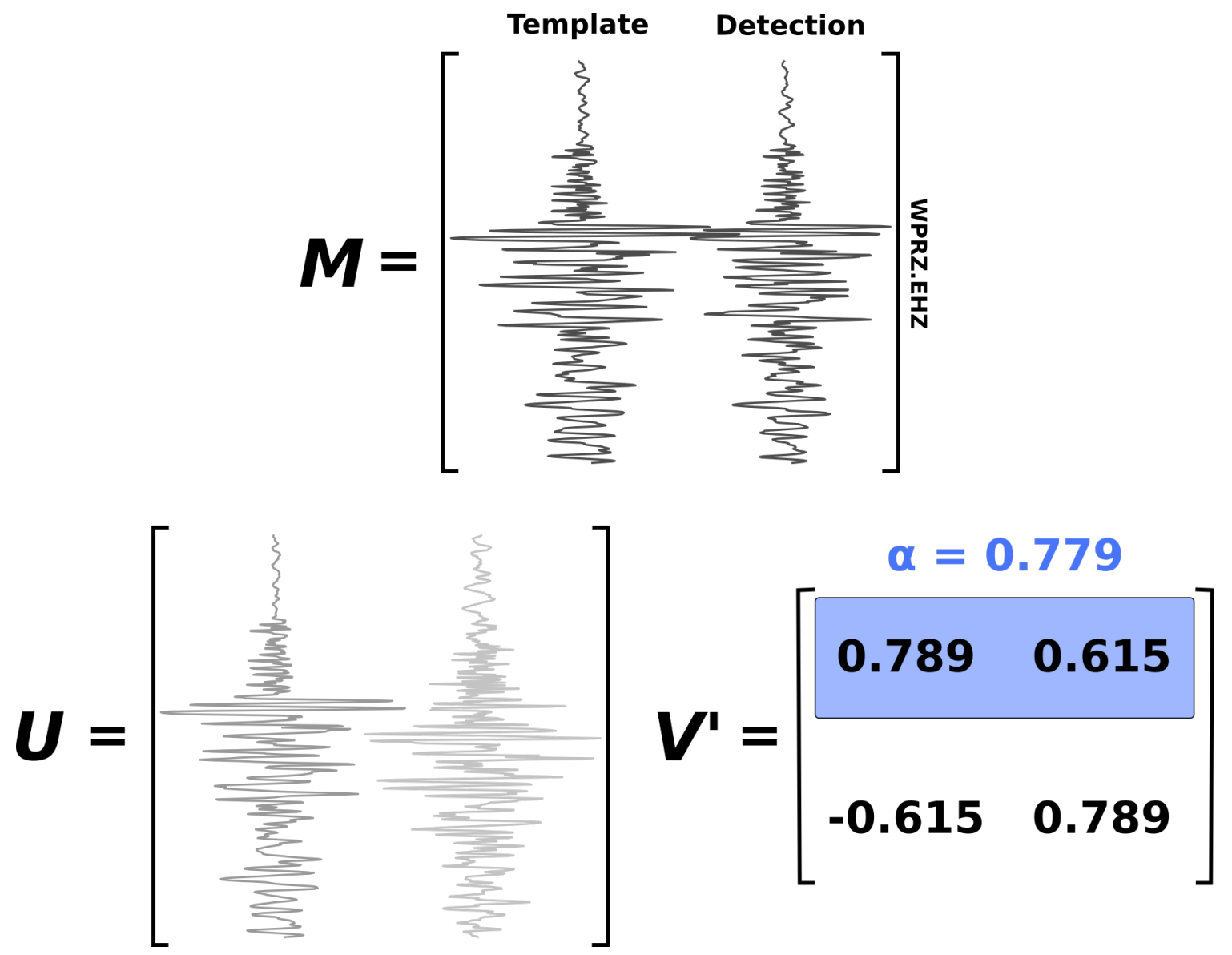

Figure 2.13: Illustration of the relative amplitude calculation between a template and detected event with real waveforms and values. The matrix, $\mathbf{M}$, is the data matrix, in our case with the waveforms as columns. The singular value decomposition comprises the left singular vectors (as columns of $U$ ), the corresponding eigenvalues, $(\Sigma$; not shown) and the right singular vectors (as rows of $V^{\prime}$ ). The columns of $U$ effectively describe the signal that both the template and detection have in common, with decreasing importance from left to right. The rows of $V^{\prime}$ represent the contribution of the columns of $U$ to each column of $M$. As the template and detected waveforms are inherently similar, the first column of $U$ describes the bulk of their signals and therefore the first row of $V^{\prime}$ describes their relative amplitudes.

determined for locally detected, shallow New Zealand earthquakes and then converting to seismic moment using the equation (Hanks and Kanamori, 1979):

$$
M_{w}=\frac{2}{3} \log _{10} M_{0}-9
$$

Knowing the relative moment of the template event from the procedure outlined above, we then determine the relationship between the relative moments and actual moment which allows us to convert relative moments to $M_{w}$ and then back to $M_{L}$ using the relationship of Ristau et al. (2016). 


\section{$b$-value}

The frequency-magnitude relationship for earthquake catalogs is a power-law distribution described by Gutenberg and Richter (1942) as

$$
\log N=a-b M
$$

where $a$ increases with the number of earthquakes in the catalog, $b$ describes the distribution of earthquake magnitudes above the catalog magnitude of completeness $\left(M_{c}\right)$ and $N$ is the number of events larger than magnitude $M$.

The Gutenberg-Richter distribution appears to hold from small-scale laboratory experiments (Scholz, 2015) to the full catalog of global seismicity (Boettcher et al. 2009), indicating fractal scaling of the earthquake rupture process. The global earthquake catalog, as well as many regional catalogs, exhibits a $b$-value of $\sim 1.0$, however $b$-values are often observed to vary spatially, with $b<1$ signifying a relatively higher proportion of large-magnitude events and $b>1$ indicating a higher proportion of smallmagnitude events (Stein and Wysession, 2003). High $b$-values are observed in volcanic areas characterized by swarms of seismicity with no discernible mainshock (e.g. Wiemer and McNutt, 1997; Wiemer et al., 1998) and in areas of high material complexity (Langenbruch and Shapiro, 2014), while areas of low b-value have been attributed to the presence of asperities on fault zones (Wiemer and Wyss, 1997). It has been shown

in the lab (Scholz, 2015) and over regional scales (Spada et al., 2013, Schorlemmer et al. 2005) that $b$ is inversely proportional to the differential stress applied to the volume of consideration and therefore might be useful as a stress meter in the crust. However, the physical mechanisms driving the scale invariance of earthquake rupture are still debated. Langenbruch and Shapiro (2014) show that the fractal scaling of the elastic properties of rock, as observed in borehole logs, leads to self similar scaling of the Coulomb stress, fracture size and, therefore, earthquake magnitude. They also show that the stress dependence of $b$ may solely be the effect of characteristic scales of earthquake processes, and may vanish with increasing size of the volume considered.

We calculate $M_{c}$ for the catalogs presented here following the methodology of Wiemer (2000). For a range of $M_{c}$ values, $a$ and $b$ are determined for our earthquake catalog and an idealized synthetic distribution constructed with the same $a$ and $b$ values. The absolute difference between the synthetic and measured distributions given by Wiemer (2000) is:

$$
R\left(a, b, M_{i}\right)=100-\left(\frac{\sum_{M_{i}}^{M_{\max }}\left|B_{i}-S_{i}\right|}{\sum_{i} B_{i}} 100\right)
$$

where $M_{\max }$ is the maximum observed magnitude and $B_{i}$ and $S_{i}$ are the observed and predicted number of events in a given magnitude bin, $i$. This residual is then minimized to find the most appropriate $M_{c}$ (Figure 4.2). 


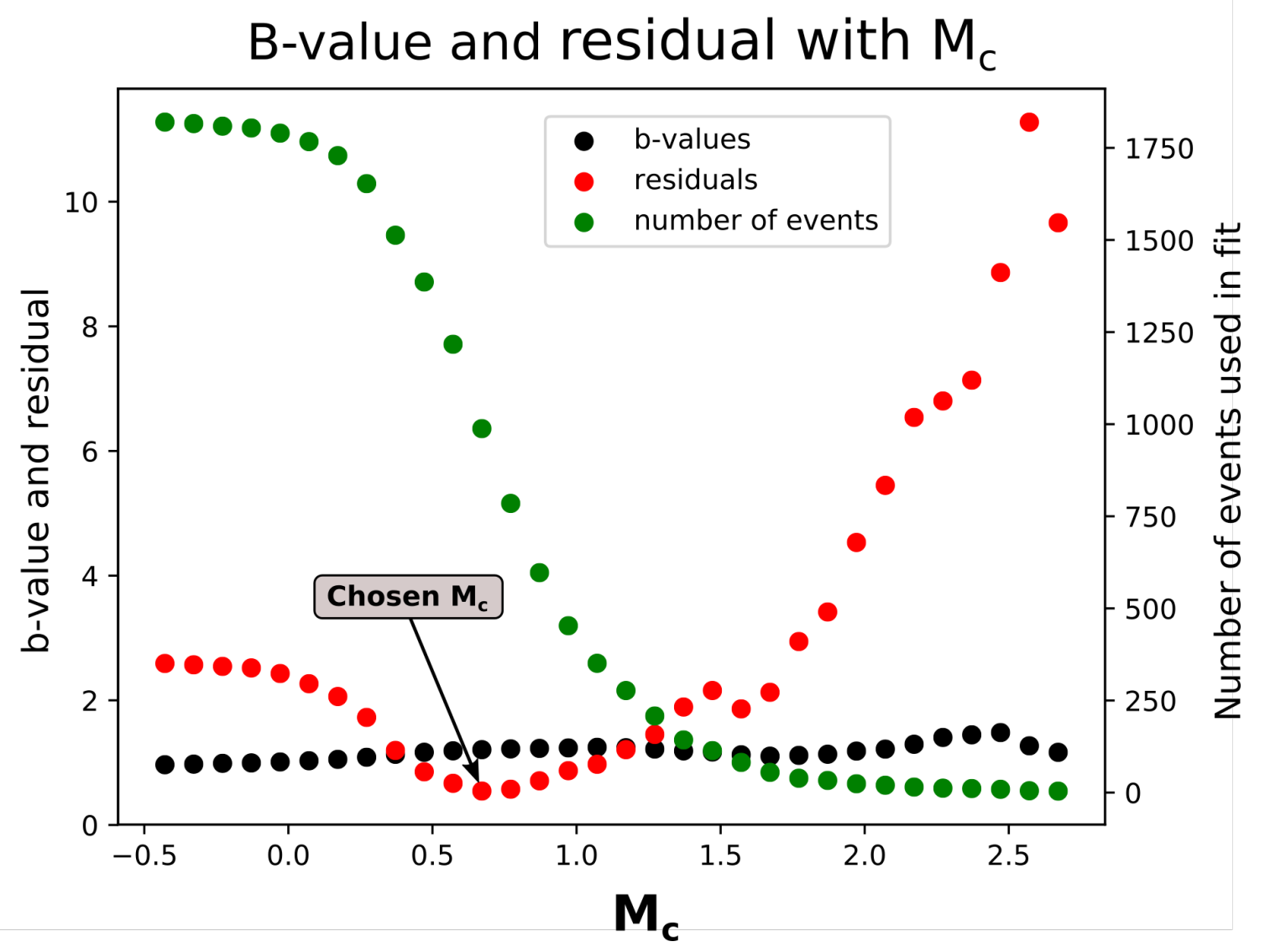

Figure 2.14: Illustration of the magnitude of completeness calculation developed by Wiemer (2000). Red dots indicate the normalized, absolute difference between the observed and theoretical distribution of events for a given $\mathrm{M}_{c}$ and $b$-value. Black dots are the calculated $b$-value and green shows the total number of events used for the fit at each magnitude of completeness.

In Chapter 4, we map b-value in space using the approach described by Bachmann et al. (2012), wherein, for each event in a catalog (which Bachmann et al. (2012) refer to as a 'focus'), the nearest $n$ events are selected and $M_{c}$ is calculated for this subset. If the number of events larger than $M_{c}$ exceeds a threshold, the $b$-value is calculated and mapped to the focus event's location using the maximum-likelihood method $A k i$, 1965; Shi and Bolt, 1982). Bachmann et al. (2012) map the $b$-value for the 150 nearest events, with a threshold of 25 events greater than $M_{c}$. Here we use the nearest 300 events with a minimum threshold of 100 events above $M_{c}$.

\subsubsection{Focal mechanisms}

The final step taken in developing the earthquake catalog presented here is the determination of earthquake focal mechanism solutions. The shear displacement on a fault is often represented as a point acted on by two perpendicular force couples (the so-called double-couple source), by ignoring any potential volumetric changes associated with 
the event (Stein and Wysession, 2003). The energy radiated by such a double-couple source can be divided into four quadrants: two dilational and two compressional. At a given seismic station, the ground displacement from an arriving $\mathrm{P}$-wave depends on which of the quadrants the path between the source and receiver emanated from. If that path emanated from a compressional quadrant the ground will move upwards, and if the path emanated from a dilational quadrant, the ground will move downwards. Therefore, by observing the pattern of first motion polarities (up or down) recorded across a seismic network, constraints can be placed on the orientation of the fault that slipped and the direction of slip associated with the corresponding earthquake Stein and Wysession, 2003).

We manually picked the P-wave arrival polarities for each template event at Rotokawa and Ngatamariki. This process was somewhat subjective as the decision to pick a polarity was based on our perception of the arrival's impulsiveness and the uncertainty of the polarity. Where we deemed the onset to be too uncertain, a polarity was not picked. For the focal mechanism calculation, we discarded all events with fewer than five polarity picks.

We checked station polarity consistency by observing the arrival polarities of distant regional events (e.g. from the Tonga/Kermadec Subduction Zone), which were expected to show the same polarity for all stations in our relatively small-aperture network (Figure 2.15). This process also served to verify the relative polarity of different instruments in our network, most of which were $4.5 \mathrm{~Hz}$ geophones that we expected to conform to a right-handed coordinate system (positive signal represents downward displacement), in contrast to the SEED standard for GeoNet stations (left-handed system where upwards displacement produces a positive signal). Figure 2.15 is an example polarity check that confirmed our suspicions of each station's polarity. The polarities of the geophones were then flipped to conform to the SEED standard prior to picking.

A number of approaches have been developed to invert polarity recordings for the fault plane orientation of an earthquake, typically by forward modeling a large number of possible sources to find the mechanism that best fits the polarity observations (e.g. Reasenberg and Oppenheimer, 1985, Hardebeck and Shearer, 2002). The focal mechanism solutions presented in this thesis were calculated using a Bayesian approached developed by Walsh et al. (2009), which, unlike previous approaches, allows for the incorporation of estimated uncertainties in the input parameters Specifically, uncertainties in the hypocentral location of the earthquakes are incorporated in the form of a probability density function (pdf output by the location algorithm NonLinLoc (Lomax et al., 2014). The hypocentral pdf can then be mapped to pdfs of the take-off angles for each ray travelling between the source and each recording station, which are then used to constrain the source focal mechanism. Further priors that are incorporated include the probability of a mispicked polarity reading, for which we use a conservative value of 0.2 , and amplitude noise in the data $(1 / 6)$ Walsh et al. $(2009)$. 

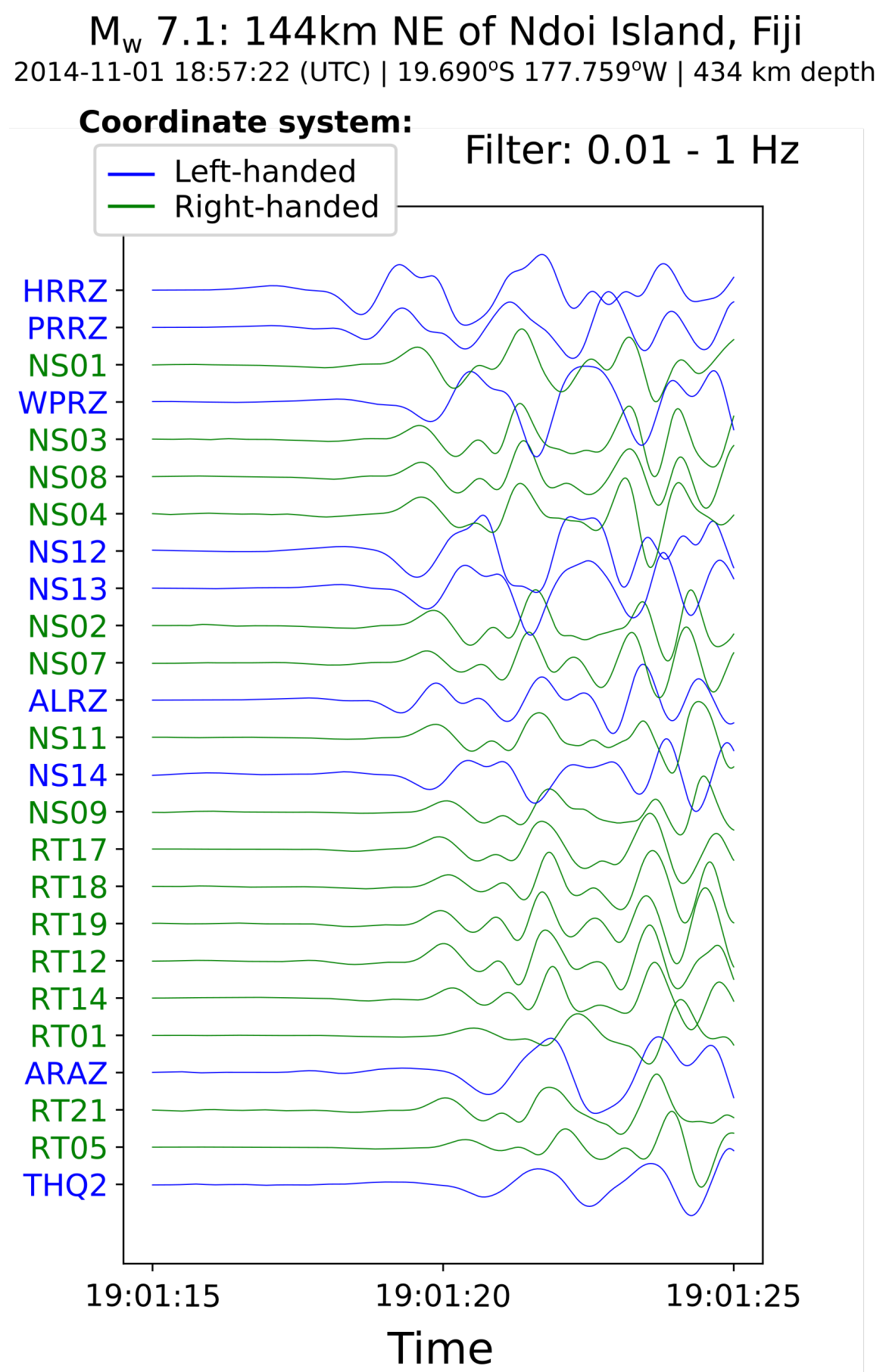

Figure 2.15: P-arrival waveforms for a deep, subducted plate event beneath Fiji at each available station in the Mercury network. In theory, all traces should exhibit the same polarity for such a distant event, although it is evident from the figure that, due to differences in their construction, they do not. Traces and station labels are color coded to their respective coordinate system such that GeoNet and borehole stations conforming to a 'left-handed' coordinate system are blue and Mercury geophones conforming to a 'right-handed' coordinate system are green. Individual traces were filtered from 0.01 to $1 \mathrm{~Hz}$ and normalized to a maximum amplitude of 1.0 for the purposes of this plot. The geophone polarities were flipped into agreement with the IRIS SEED standard (left-handed coordinates) before polarities were picked for the Rotokawa and Ngatamariki catalogs. 
An example plot of the take-off angle and resulting focal mechanism parameter pdfs is shown in Figure 2.16 .

\section{Template 2013sora337975}
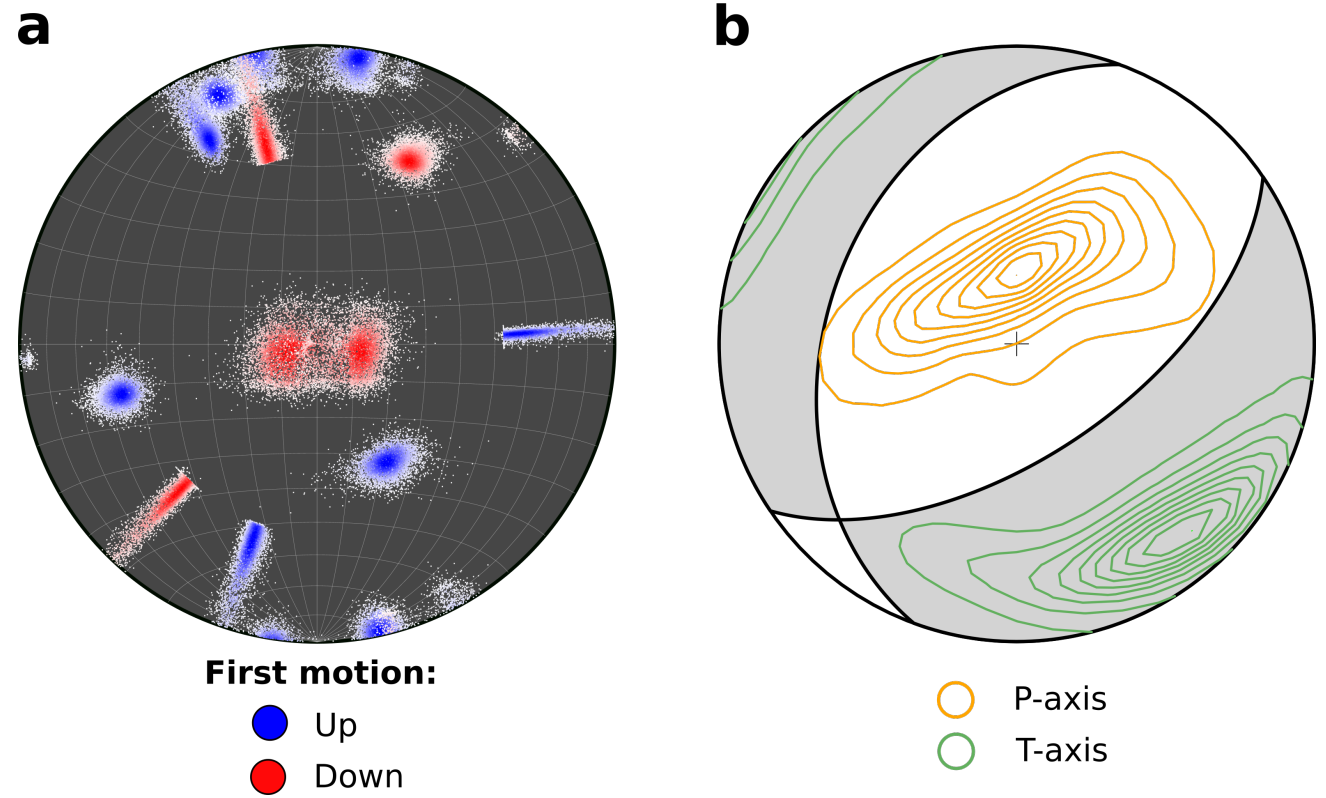

Figure 2.16: a) Sampled take-off angle pdf for a single Rotokawa template event with blue samples representing an upward first arrival polarity and red samples representing a downward polarity. b) Contoured posterior pdfs for the $\mathrm{P}$ (orange) and $\mathrm{T}$ (green) axes calculated for the polarities in a) using the approach of Walsh et al. (2009).

\subsubsection{Stress inversion}

Displacement along a fault or fracture in the Earth's crust (e.g an earthquake) allows an observer to make an inference about the state of stress that allowed that movement to occur. A single earthquake cannot fully constrain the stress field that produced it, however by considering a suite of focal mechanism solutions it becomes possible to estimate the state of stress that best fits all of the observations simultaneously. During the last several decades, a number of approaches have been used to invert focal mechanism observations for the stress state (e.g. Michael, 1984; Gephart and Forsyth, 1984; Hardebeck and Michael, 2006; Martínez-Garzón et al., 2014). In turn, these methods have been applied to a variety of tectonic environments (e.g. Michael, 1987, 1991; Townend and Zoback, 2001; Hardebeck and Michael, 2004; Townend and Zoback, 2006; Schoenball et al., 2012; Townend et al., 2012; Martínez-Garzón et al., 2013; Warren-Smith et al., 2017).

Focal mechanism stress inversion is built upon the assumption of the 'Wallace-Bott hypothesis', which states that, within a uniform stress field, two rigid blocks of crust will slip past one another in the direction of the maximum resolved shear stress on the fault 
that separates them (Wallace, 1951; Bott, 1959; McKenzie, 1969). Typically, a group of observed focal mechanism slip vectors is then inverted for the best fitting stress field, with additional focal mechanism observations further constraining the orientation of the stress tensor. One important caveat is that all observations cannot be highly-similar (as is often the case near large plate-bounding faults), in which case the maximum and minimum magnitude axes of the resulting stress tensor will simply reflect the input $\mathrm{P}$ and $\mathrm{T}$ axes of the input focal mechanisms (Townend and Zoback, 2001; Arnold and Townend, 2007).

Here we use a Bayesian approach developed by Arnold and Townend (2007) for which the focal mechanism code of Walsh et al. (2009), mentioned above, was designed to be used as input. Arnold and Townend (2007) derived a formulation for the posterior distribution of the stress state, described by three Euler angles (in geographic coordinates), $\Phi_{S}$, and a stress shape parameter $\nu$

$$
\nu=\frac{\sigma_{1}-\sigma_{2}}{\sigma_{1}-\sigma_{3}}
$$

where $\sigma_{1}, \sigma_{2}$ and $\sigma_{3}$ are the maximum, intermediate and minimum-magnitude axes of compressive stress. Given $n$ number of focal mechanism observations, $\Phi_{G i}^{0}$, with precision $\tau_{i}$

$$
p\left[\Phi_{S}, \nu \mid \Phi_{G i}^{0}, \tau_{i}\right] \propto \sin \Theta_{S} \prod_{i=1}^{n} \sum_{l=1}^{2} q\left(\Phi_{S i}^{(l)}, \nu \mid \tau_{i}\right)
$$

The formulation above effectively states that the posterior distribution of the stress state parameters is the product of the posterior distributions of each of the $n$ focal mechanism observations, here expressed in stress coordinates, $\Phi_{S i}^{(l)}$, and accounting for the ambiguity of the nodal plane of each focal mechanism by summing over both, finally multiplied by the factor $\sin \Theta_{S}$. The final step in the stress inversion process involves projecting the resulting stress state distribution onto a coordinate system in which one of the principal stress axes is vertical and calculating the posterior distribution of the axis of maximum horizontal compression, $\mathrm{S}_{\text {Hmax }}$. Figure 2.17 shows the contoured posterior $\overline{p d f}$ for $\sigma_{1}$ (red), $\sigma_{2}$ (green) and $\sigma_{3}$ (blue) for a cluster of events in Ngatamariki. 

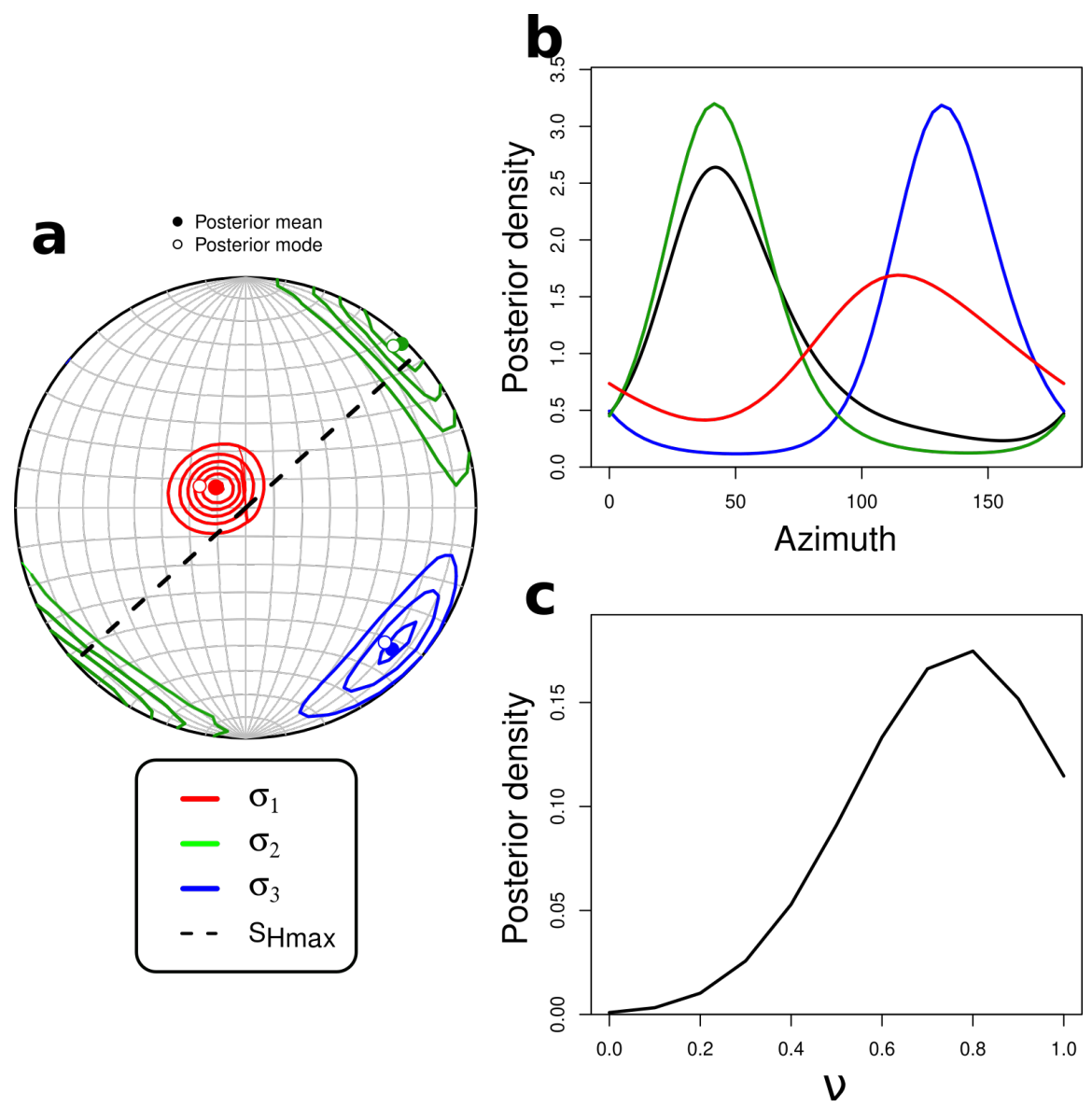

Figure 2.17: Example stress inversion results for a subset of focal mechanisms from Rotokawa. a) Lower hemisphere projection of the contoured 3-dimensional posterior stress tensor pdf. Red corresponds to $\sigma_{1}$, green to $\sigma_{2}$ and blue to $\sigma_{3}$. b) Marginal posterior pdfs showing the azimuth of the principal stress axes as well as $\mathrm{S}_{\text {Hmax }}$ (black). c) Posterior pdf for the shape parameter, $\nu$. 


\title{
3 Seismic response to injection well stimulation in a high-temperature, high-permeability reservoir
}

\begin{abstract}
Fluid injection into the earth's crust can induce seismic events that cause damage to local infrastructure but also offer valuable insight into seismogenesis. The factors that influence the magnitude, location and number of induced events remain poorly understood but include injection flow rate and pressure as well as reservoir temperature and permeability. The relationship between injection parameters and injection-induced seismicity in high-temperature, high-permeability reservoirs has not been extensively studied. Here we focus on the Ngatamariki geothermal field in the central Taupo Volcanic Zone, New Zealand where three stimulation/injection tests have occurred since 2012. We present a catalog of $>3700$ microearthquakes from 2012-2015 created using a matched-filter detection technique. We invert first-motion-derived focal mechanisms for the stress state, yielding an average direction of maximum horizontal compressive stress $\left(\mathrm{S}_{\mathrm{Hmax}}\right)$ of $\sim 040^{\circ}$, consistent with the regional NE-SW trend. The maximum compressive stress $\left(\sigma_{1}\right)$, plunging $\sim 30^{\circ}$ in the northern injection zone, is subvertical in the southern injection zone, which may reflect geological differences between wells. Using the ratio of injection flow rate to overpressure (injectivity index as a proxy for near-well permeability, we show that seismicity and permeability increase are not closely related processes, as is commonly assumed.For the case of one injection stimulation treatment, seismicity lags behind injection by more than eight days, during which time the injectivity index nearly doubles. Therefore, we infer that permeability change is driven by thermo- and poroelastic effects, while seismic slip does not measurably affect the permeability experienced at the wellbore. Our findings augment a growing body of work suggesting that aseismic opening or slip, rather than seismic shear, is the active process driving borehole stimulation in many environments.
\end{abstract}




\subsection{Introduction}

In recent years, the number of recorded cases of injection-induced seismicity has grown dramatically with the proliferation of industrial activities such as wastewater disposal and enhanced geothermal systems (Ellsworth, 2013). In many cases, most notably in the central US and Europe, these activities have induced events that caused damage to local infrastructure and even a number of injuries (e.g. Keranen et al., 2013, Hsieh and Bredehoeft, 1981; Deichmann and Giardini, 2009; Kim et al., 2018; Grigoli et al. 2018; Ge et al., 2019). However, these injections also offer valuable insight into seismogenesis, which may help to better manage future injection-induced seismic hazard. While most case studies have addressed low-temperature, high-permeability reservoirs such as the Arbuckle group in Oklahoma (e.g. Langenbruch and Zoback, 2016) or medium-temperature, low-permeability reservoirs targeted at enhanced geothermal system (EGS) sites (e.g. Deichmann and Giardini, 2009, Evans et al., 2005, Grigoli et al. 2018), here we present a case of induced seismicity related to injection operations in the high-temperature, high-permeability Ngatamariki geothermal reservoir. Though seismicity associated with such reservoirs has been observed for decades (for example, at The Geysers field in California; Allis, 1982; Oppenheimer, 1986), most studies have needed to consider simultaneous injection from multiple wells with long injection histories. This has complicated the relationship between induced seismicity and injection parameters such as flow rate and wellhead pressure (WHP) (e.g. Martínez-Garzón et al., 2013; Boyle and Zoback, 2014; Martínez-Garzón et al., 2014; Kwiatek et al., 2015; Martínez-Garzón et al., 2017). Here we present a simpler case study involving a small number of injections isolated from one another in both space and time.

The aim of underground fluid injection is typically either to dispose of unwanted fluids or to increase permeability at depth (Ellsworth, 2013; Grant and Bixley, 2011). In the geothermal industry, an increase in permeability allows for more fluid to be injected or produced, reducing the number of wells required per unit of electrical generation. Fluid injection drives a number of processes that contribute to the occurrence of seismicity, including pore-fluid pressure increases, reservoir volume changes (due to injection or extraction of fluid), reservoir temperature decreases and chemical changes to fracture surfaces (Majer et al., 2007). The conditions under which these different processes induce seismicity and the relationship between seismic or aseismic slip and reservoir properties such as fracture permeability remain unclear Amann et al., 2018. Das and Zoback, 2011, and references therein). Unraveling these potential relationships is important in order to better plan future geothermal resource development, and has implications for deep injection operations and the understanding of seismogenesis in general. 
The Ngatamariki geothermal field in the central Taupō Volcanic Zone of New Zealand is a convenient place to study induced seismicity. Prior to our study period, which extends from June 2012 until the end of 2015, the development of the Ngatamariki resource had been limited to resource exploration in the 1980s followed by the drilling of three deep exploration wells (NM05, NM06 and NM07 to $\sim 3 \mathrm{~km}$ depth) in the 2000s (Chambefort et al., 2016). The start of injection operations in 2012 represented the first large-scale fluid injection into the undisturbed, high-temperature $\left(\sim 280^{\circ}\right)$ Ngatamariki reservoir (Bignall, 2009$)$. Fluid injection at three of the four major injection boreholes (NM08, NM09 and NM10) was undertaken in advance of the Ngatamariki power plant's commissioning in 2013. Pressure and flow rate during each of these operations were systematically-recorded ( $5 \mathrm{~min}$ resolution). As each operation occurred in isolation, the task of relating temporal and spatial patterns of microseismicity to injection parameters for a specific well was straightforward. Seismic data were recorded throughout these injections, enabling us to detect and precisely locate a large number of induced microearthquakes.

In the Ngatamariki case, the objectives of individual injection operations differed. Generally, these injections can be divided into three categories: cold-water stimulation, injection testing, and unintended fluid losses during drilling (Clearwater et al., 2015). Cold-water stimulation is a process intended to increase the permeability in a given well, and is thought to be driven by the thermal contraction of reservoir rocks Grant et al., 2013). Injection testing, while often conducted in conjunction with stimulation, is aimed specifically at determining the injectivity index of a well, which is normally defined as the ratio of flow rate to wellhead pressure. This parameter is then used as an indicator of a well's bulk permeability and to predict injection well performance once a power plant begins operation (DiPippo, 2016). Fluid losses during drilling are generally unintended and result from the escape of drilling fluid into the formation as the well is drilled. Each of these scenarios occurred at Ngatamariki prior to the startup of the 82 Megawatts electrical MWe power plant in April of 2013 Clearwater et al. 2015). As we show below, the rate, location and magnitude of microseismicity associated with each individual injection operation were distinct, revealing differences in local geology, near-well permeability, fluid injection rate, history of injection and the temperature and chemistry of the injected fluid in each well.

In this paper, we construct a catalog of induced seismicity at Ngatamariki and compare the characteristics of seismicity to flow rate, pressure and injectivity index measured during three phases of injection prior to the startup phase of the Ngatamariki power plant. The temporal isolation of each phase of injection allows us to relate nearwell seismicity to high-resolution injection parameters without contamination from multiple, concurrent injections. Given that the Ngatamariki reservoir occupies the upper end of both the temperature and permeability continuum, the injection-seismicity relationship here serves as a useful comparison to results from lower-temperature and 
lower-permeability settings elsewhere. Therefore, our results help to distinguish the contributions and relative importance of reservoir permeability and temperature in forecasting the extent and magnitude of induced seismicity during future injection operations.

\subsubsection{Geological and geophysical setting}

The Ngatamariki geothermal field is located in the central Taupō Volcanic Zone (TVZ) on the North Island of New Zealand, approximately $17 \mathrm{~km}$ north of the town of Taupo (Figure 3.1). Ngatamariki is a high-temperature, liquid-dominated and naturallyfractured system $\left(280^{\circ} \mathrm{C}\right.$ at depths exceeding $\left.1000 \mathrm{~m}\right)$ that measures roughly $5.5 \mathrm{~km}$ from north to south and $3 \mathrm{~km}$ from east to west (Bignall, 2009, Chambefort et al., 2014). The reservoir is hosted in a succession of volcaniclastics known as the Tahorakuri Formation, which exhibits significant lateral heterogeneity (Chambefort et al., 2014). In the south, the deeper portions of the reservoir ( $<2000$ meters below sea level) are hosted in the Rotokawa Andesite. In this part of the field, the Rotokawa Andesite overlies greywacke basement, which has been encountered in only one well (NM06) at $3012 \mathrm{~m}$ below sea level (bsl; Chambefort et al., 2014). The geologic structure of the southern end of the field, between the central production wells and injection wells NM06 and NM10, is dominated by the active, NE-SW-striking Aratiatia Fault Zone (Figure 3.1). Reservoir tracer tests have demonstrated the presence of high-permeability between injection well NM10 and production well NM05 along an Aratiatia Fault Zone-related structure (Buscarlet et al., 2015). In the north, in the vicinity of injection wells NM08 and NM09, the reservoir geology is dominated by a shallow intrusive body $(<2000 \mathrm{~m}$ bsl), the presence of which was confirmed by drill cuttings and core in NM08, NM09 and NM04 (Bignall, 2009; Chambefort et al., 2014). The intrusive body lacks appreciable permeability itself $\left(\sim 10^{-18} \mathrm{~m}^{2} ;\right.$ Cant et al., 2018$)$, but it is enveloped by a highlyfractured damage zone that was encountered in wells NM08 and NM09 Clearwater et al., 2015). Given the low permeability of the reservoir matrix, typical of the TVZ (Sibson and Rowland, 2003), fluid flow is controlled by fractures and faults. Regionally, faults follow a NE-SW structural trend and borehole image logs at Ngatamariki indicate mostly NE-SW-oriented fractures within the reservoir, with some variability with depth in certain wells (Bignall, 2009; Massiot et al., 2015b, 2012).

\subsubsection{Mechanisms of microseismicity and permeability enhance- ment}

The main driver of microseismicity at Ngatamariki is the injection of cool $\left(<100^{\circ} \mathrm{C}\right)$ fluid into the hot $\left(\sim 280^{\circ} \mathrm{C}\right)$ reservoir. It is generally accepted that fluid injection increases the pore-fluid pressure near an injection well, lowering the effective normal 


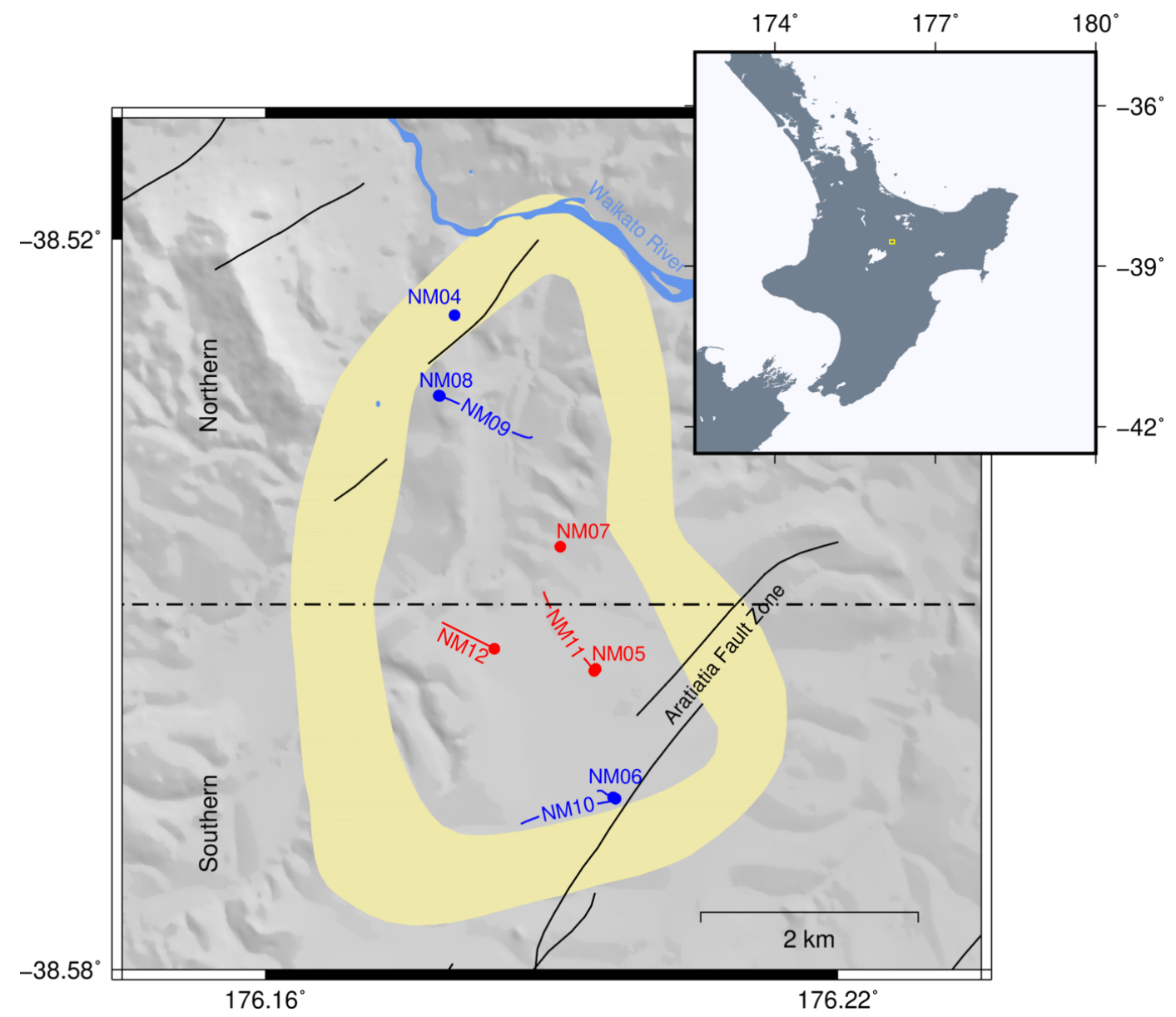

Figure 3.1: Overview of the Ngatamariki geothermal field. Injection wells are shown in blue, production wells in red with dots representing the wellhead and lines showing the surface projection of the well tracks at depth. Wells NM04, NM08, NM05 and NM07 are near vertical and, therefore, appear only as dots in the figure. Active faults from the GNS Science Active Faults Database (Langridge et al., 2016) are shown in black. The most likely boundary of the deep resource as described by Boseley et al. (2010) based on magnetotelluric surveys, is shown in yellow. The northern and southern portions of the field, as referred to in this work, are divided at -38.55 degrees latitude and labeled here.

stress and inducing slip on fractures suitably oriented with respect to the local stress field (e.g. Zoback and Harjes, 1997; Ellsworth, 2013; Langenbruch et al., 2018). At every point on a fracture or fault, there is a specific pore-pressure increase, $\Delta P_{\text {crit }}$, that will lower the shear strength of the fracture/fault to the point of failure Wiprut and Zoback, 2000). Injection, especially in high-temperature geothermal reservoirs, also introduces a thermal gradient in the host rock. This may produce enough stress to induce tensile failure or opening of preexisting fractures (Martínez-Garzón et al., 2014). It also reduces stress locally through thermoelastic contraction of the rock matrix, bringing the reservoir fracture network closer to, or further from, failure depending upon the direction of fluid flow relative to the orientation of the in situ stress state (Jeanne et al., 2014). Furthermore, as geothermal fluid moves through a formation, 
it may dissolve minerals (e.g. calcite) out of, or precipitate minerals into, fractures. Though poorly understood, this effect may play a large part in creating or destroying permeability through opening or sealing of fractures that would otherwise be likely to fail (Clearwater et al., 2015). Injection and extraction of large quantities of fluid can also significantly change the volume of the reservoir, poroelastically influencing the distribution of stress and often leading to reservoir compaction, surface subsidence and seismicity Segall, 1989; Segall and Fitzgerald, 1998; Bromley et al., 2013). Finally, slip itself (both seismic and aseismic) transfers stress onto nearby fractures and faults, an effect that has been shown to play a role in triggering subsequent seismicity in geothermal fields and elsewhere (Schoenball et al., 2012; Catalli et al., 2016; Cappa et al., 2019).

The relationship between seismicity and permeability has been widely studied in laboratory settings (e.g. Lee and Cho, 2002, and references therein). When slip occurs on a new or preexisting fracture, asperities become offset and the permeability of that fracture increases (an effect known as self-propping), which depends on the amount of slip and the roughness of the fault surface (Ishibashi et al., 2018; Esaki et al., 1999; Fang et al., 2017). It is commonly assumed that the permeability increase observed during injection is the result of seismic fault slip, a correlation which has been modeled for EGS cases previously (e.g. Baisch et al., 2010). It is important to note, however, that a large percentage of the permeability enhancement that accompanies borehole stimulation may be aseismic, as has been directly observed during a decameter-scale injection experiment described by Guglielmi et al. (2015) and in seismic data recorded during hydraulic fracturing (Das and Zoback, 2011). Further evidence of aseismic slip has been inferred at the high-temperature Salton Sea geothermal field in southern California from a combined geodetic and seismic dataset (Wei et al., 2015). Riffault et al. (2018) have also demonstrated that, in some cases, permeability enhancement may not be coupled to detectable seismic slip at all. In the context of high-temperature geothermal reservoirs, thermal stresses induced near the wellbore may govern most of the permeability enhancement observed during well stimulation through the expansion of permeable zones (Grant et al., 2013, Siega et al., 2014), a process which may be aseismic.

At Ngatamariki, the dominant processes driving microseismicity have previously been interpreted to be thermal and pore-fluid pressure changes Sherburn et al. 2015 . Grant et al. 2013). The Ngatamariki power plant reinjects most of the extracted fluid and, as a result, the pressure drawdown observed across the field is minor $(\sim 0.2$ $\mathrm{MPa}$ ). This suggests that reservoir compaction plays a small role in inducing local stress changes in this case (Quinao et al. 2017), whereas at nearby fields much longer production histories (i.e. Wairakei), significant pressure drawdown-related subsidence has occurred (Allis, 2000). Poroelastic stress transfer resulting from rock matrix contraction and slip on fractures cannot be ruled out as possible factors affecting reservoir 
permeability and induced seismicity. Also, in the case of the NM08 and NM10 injection tests and NM09 stimulation A (introduced below), the injection of river water also renders unlikely the precipitation of minerals as a possible mechanism of permeability change. This is unlikely to be the case for the brine injected during the second phase of NM09 stimulation.

\subsubsection{Ngatamariki power plant operations}

Mercury NZ Ltd., then known as Mighty River Power, began generation of electricity at Ngatamariki in October 2013 with the commissioning of an 82 MWe binary power plant. The company was granted a consent for production of 60,000 tons of geothermal fluid per day, approximately $98 \%$ of which is currently reinjected into the deep reservoir at between 1000 and $3000 \mathrm{~m}$ depth. The reinjected fluid is allocated nearly evenly between the injection wells to the north (NM08, NM09) and those to the south (NM06, NM10) (Clearwater et al., 2015; Buscarlet et al., 2015). Between June 2012 and April 2013, three of the four main injection wells were subject to some form of injection operation (Table 3.1). Cold-water stimulation of NM08 took place between 8 June and 10 July, 2012 using $\sim 10^{\circ} \mathrm{C}$ river water. Well NM10 was drilled between late May and early August 2012, using fluid consisting almost entirely of river water, and significant fluid losses occurred after 13 July. After the completion of drilling, NM10 injection testing was conducted on 1-23 September. NM09 was drilled between September and early November 2012, with subsequent injection testing occurring in two phases. The first took place between 14 December, 2012 and 4 January, 2013 and the second lasted from 13 February, to 6 March, 2013 (Clearwater et al., 2015), the latter using geothermal brine instead of river water. This paper focuses on the cold-water stimulation of NM08, drilling and cold-water stimulation of NM10 and cold-water stimulation of NM09.

In March 2013, as the plant was being brought online, Mercury began reinjection of brine into all injection wells including NM06, which had been drilled several years earlier. Geothermal brine at Ngatamariki is injected at a temperature of approximately $90^{\circ} \mathrm{C}$. However, at times when production exceeded plant intake capacity during the early stages of plant startup, some brine bypassed the plant and was injected at temperatures of as high as $150^{\circ} \mathrm{C}($ Clearwater et al., 2015). Over the following year, injection at Ngatamariki reached a stable level of $\sim 1000$ tonnes per hour (t/h) at NM09, 200 $\mathrm{t} / \mathrm{h}$ at NM08 and $\sim 800 \mathrm{t} / \mathrm{h}$ at NM06. NM10 was initially an active injector but, due to the strong hydraulic connectivity between it and the production well NM05, it was phased out by mid-2015 (Buscarlet et al. 2015). 


\begin{tabular}{|c|c|c|c|c|c|c|}
\hline Operation & Zone & Start & End & Injectate & $\operatorname{Max} Q(\mathrm{t} / \mathrm{h})$ & Max Pres. (MPa) \\
\hline NM08 Stimulation & Northern & $2012-6-8$ & $2012-7-10$ & River water & 175 & 2.63 (WHP) \\
\hline NM10 Drilling & Southern & $2012-5-25$ & $2012-8-11$ & Drilling fluid & 141 & $\mathrm{~N} / \mathrm{A}$ \\
\hline NM10 Stimulation & Southern & 2012-9-1 & 2012-9-23 & River water & 201 & $9.3(\mathrm{DHP})$ \\
\hline NM09 Stimulation A & Northern & 2012-12-14 & 2013-1-4 & River water & 170 & 0.2 (WHP); 15.3 (DHP) \\
\hline NM09 Stimulation B & Northern & $2013-2-13$ & $2013-3-6$ & Brine & 152 & 0.3 (WHP); 13.0 (DHP) \\
\hline
\end{tabular}

Table 3.1: Table summarizing the injection operations presented here, all of which were undertaken prior to plant startup at Ngatamariki. The maximum pressures reported in the final column are for wellhead pressure (WHP), downhole pressure (DHP) or both where present. Q denotes flow rate in tonnes per hour.

\subsection{Data}

The Mercury seismic network covers an area roughly $30 \mathrm{~km}(\mathrm{~N}-\mathrm{S})$ by $15 \mathrm{~km}(\mathrm{E}-\mathrm{W})$ with the majority of the stations occupying an area $15 \mathrm{~km}(\mathrm{~N}-\mathrm{S})$ by $7 \mathrm{~km}(\mathrm{E}-\mathrm{W})$ spanning the Rotokawa and Ngatamariki geothermal areas (Figure 2.1). The majority of the instruments are either $4.5 \mathrm{~Hz}$ Geospace GS-11D short-period geophones or Lennartz LE-3DLite $1 \mathrm{~Hz}$ instruments, but the network also includes stations operated by Contact Energy (THQ2, ARAZ) at the Wairakei and Tauhara geothermal fields and nearby stations operated by the national seismic network, GeoNet (WPRZ, PRRZ, HRRZ, ALRZ) (Table 2.1). The GeoNet stations are broadband instruments with the exception of WPRZ, which is a $1 \mathrm{~Hz}$ LE-3Dlite. From the beginning of 2012 until the end of 2015, the number of operational stations varied between 15 and 29 . Sites NS12, NS13 and NS14 in the middle of the Ngatamariki network are $2 \mathrm{~Hz}$ borehole instruments installed at depths of between 200 and 514 meters below the ground surface (Figure 2.1).

The initial earthquake catalog for this study was provided by GNS Science under contract to Mercury. The waveform data were collected roughly every three months from Mercury's data loggers and supplemented by data from nearby GeoNet stations. GeoNet data were sampled at $100 \mathrm{~Hz}$ while the Mercury network data were sampled at $200 \mathrm{~Hz}$. For much of this study, all data were resampled at $50 \mathrm{~Hz}$ to reduce computational costs. Events in the initial GNS Science earthquake catalog had been automatically detected and located with the SeisComP3 software package (Weber et al., 2007). We filtered the catalog to include only those events within or immediately adjacent to the field boundaries at Ngatamariki, leaving a total of 1171 microearthquakes of local magnitudes between 0.26 and 3.17. Magnitude distance corrections were computed using events recorded by both the Mercury seismic network and GeoNet. The calibration factor for a given event-station distance, $A 0$, was calculated from $M=\log _{10} A-\log _{10} A 0$, where $A$ is the event amplitude at the station and $M$ the GeoNet calculated magnitude. $A 0$ was averaged across all stations in the Mercury network for all events recorded by GeoNet to obtain the final calibration factors. A final, static correction factor of +0.32 was applied to the Mercury magnitudes 
to bring them into agreement with GeoNet-calculated magnitudes (Figure 2.3). We relocated these events using the double-difference relocation software of Trugman and Shearer (2017), the results of which are shown relative to the seismic network in Figure 3.11 (Appendices). Production and injection well locations as well as flow rates and pressures at each well were provided by Mercury.

\subsection{Methods}

\subsubsection{Matched-filter detection}

The small magnitudes of events, high levels of anthropogenic noise and highly-attenuating geology at Ngatamariki make detecting microearthquakes difficult. One way to address this difficulty is to use a waveform-correlation-based detection technique. Correlationbased detection, otherwise referred to as matched-filter detection, offers improved performance over traditional, amplitude-based techniques due to its ability to detect signals in noisy data and when multiple events are closely spaced in time (Gibbons and Ringdal, 2006; Shelly et al., 2007). This significantly increases the number of events detected without increasing the rate of false detections and is ideal for monitoring microseismicity. Such a technique is well-suited to areas of geothermal power generation, which are characterized by numerous noise sources and dense clusters of small seismic events.

Matched-filter earthquake detection relies on waveform cross-correlation of a known earthquake signal or signals, referred to as templates, with continuously recorded seismic data. The following equation, which we use for this study, describes normalized cross-correlation in the time domain (Chamberlain et al., 2017a):

$$
R(x)=\frac{\sum_{x^{\prime}=0}^{x+w_{x}}\left(T^{\prime}\left(x^{\prime}\right) \cdot I^{\prime}\left(x+x^{\prime}\right)\right)}{\sqrt{\sum_{x^{\prime}=0}^{x+w_{x}}\left(T^{\prime}\left(x^{\prime}\right)^{2} \cdot \sum_{x^{\prime}=0}^{x+w_{x}} I^{\prime}\left(x+x^{\prime}\right)^{2}\right.}}
$$

Here $I^{\prime}$ represents the continuous seismic data of interest and $T^{\prime}$ represents the template earthquake. $x$ represents the sample in the continuous data between sample 0 and sample $\left(N_{x}-w_{x}\right)$, where $N_{x}$ is the length of the continuous data being searched and $w_{x}$ is the length of the template. $x^{\prime}$ is the position within the window over which the correlation is being calculated.

Each of the 1171 events taken from the GNS Science catalog outlined in Section 3.2 was used as a template event (Figure 3.11; Appendices). Each template consists of one-second-long waveforms, starting 0.1 seconds before the P-phase arrival on the vertical channel at each station on which a P-pick was made (e.g. red waveforms in Figure 3.2). Horizontal channels are not used due to the large uncertainties for the automatic S-picks in the GNS Science catalog. However, a length of one second ensures 
that both the P- and S-arrival are included in the vertical-channel template for each event due to the short travel times. A length of one second also omits most of the coda, which is incoherent for even highly similar sources. This effect is especially apparent at Ngatamariki, due to the highly-fractured reservoir, large variations in the volcanic geology (i.e. welded ignimbrites and ashfall deposits) and surface heterogeneity. After applying an anti-aliasing filter, waveforms were sampled at $50 \mathrm{~Hz}$ and filtered from 3.0 to $20.0 \mathrm{~Hz}$ to include the high corner frequencies of events with event-station distances of $<5 \mathrm{~km}$. Continuous seismic data were processed in an identical manner to the templates for the matched-filter routine.

To generate detections, event templates were cross-correlated with continuous data at a rate of 50 samples per second. At each sample, the cross-correlation coefficients for each channel of data were summed to create the network detection statistic Shelly et al. 2007). A detection was recorded whenever the detection statistic exceeded a threshold value, which in this case was defined as the daily median absolute deviation (MAD) of the detection statistic multiplied by eight (as suggested by Shelly et al., 2007). We refer to a template event, along with all of its associated detections, as a family.

We split all families into separate groups for northern and southern Ngatamariki before removing duplicate detections. This allows us to account for simultaneous seismicity within both clusters, which we would expect in response to simultaneous injection at the two distinct injection zones. However, the separation of the two clusters prior to duplicate removal introduces the possibility of double-counting events detected by two templates if both templates are located in separate spatial clusters. We have checked for this case manually and have eliminated these cases in the final catalog presented here.

Within each spatial cluster, duplicate removal was conducted by looping through all detections in order of descending detection statistic and removing detections within a user-defined time span of two seconds. We adopted two seconds for the time span after a visual review of template events revealed numerous cases of near-repeating seismicity with inter-event times of 3-5 s.

Visual inspection of a subset of the detection waveforms showed that false detections occurred at a rate of approximately 1-3 false detections per day. However, the large number of detections makes visual review of the entire detection catalog impracticable. Therefore, we employ a sequence of thresholds based on the cross-correlation between the template and detected waveforms to exclude lower-quality events. We do this during both the location and magnitude calculations detailed below. Applying these correlation cutoffs has the effect of suppressing false detections in the final catalog that we use in later analysis. As a final quality-assurance step, we visually inspected hundreds of waveforms from the final catalog in order to manually pick first-motion polarities, and did not encounter a false detection. 


\subsubsection{Detection location}

After removing duplicate detections, P-picks for each of the newly detected events were made at each channel included in the template. For each station, template waveforms were correlated with the detected waveform over a 0.2 second window centered on the detection time. Picks were recorded at the time corresponding to the highest correlation value within that window. However, if the correlation value of the template and detected waveform fell below 0.4, the pick was discarded. We retained all events with more than five picks and discarded those with five or fewer. For the retained events, we then made automatic S-picks (Mroczek et al. 2019) using the method developed by Diehl et al. (2009) and modified by Castellazzi et al. (2015). These events were then located with the nonlinear location program NonLinLoc (Lomax et al., 2014) using a preliminary 1-D model computed in VELEST Kissling et al., 1994; Sewell et al., 2017, Table 2.3;). As a final step, the entire catalog was relocated using the doubledifference relocation program GrowClust (Trugman and Shearer, 2017) with differential pick times generated using the Python package hypoDDpy (Krischer, 2015). The final double-difference relocations are shown in Figure 3.3 .

\subsubsection{Magnitudes}

To compute the magnitude of the events detected by the matched filter, we used the method described by Shelly et al. (2016a). This technique uses pair-wise relative amplitudes between a template event and each of its detections to compute relative moments. This approach computes the relative amplitude, $\alpha$, as

$$
\alpha=\frac{v[2]}{v[1]}
$$

where $v[2]$ and $v[1]$ are the second and first elements, respectively, of the first row of the $2 \times 2$ matrix $V^{\prime}$ in

$$
M=U \Sigma V^{\prime}
$$

where $M$ is the singular value decomposition of a data matrix containing both the template and the detected waveform on a single channel. The rows of $V^{\prime}$ are the right singular vectors which map the weight of the left singular vectors, $U$, to the original data vectors. Because the template and detected waveforms in the data matrix are inherently similar, the first left singular vector, $U_{0}$, should describe only a difference in amplitude between template and detection. The relative amplitude between the two events can therefore be estimated as the ratio of the second and first elements, $v[2]$ and $v[1]$, of the first row of $V^{\prime}$.

We calculated relative amplitudes only when the cross-correlation coefficient between the template and detection exceeded 0.6 at any given station. Again, we note 
that template events only contain waveforms for the vertical channels. For those events recorded by a minimum of four stations exceeding the correlation threshold, we calculated the relative moment as the median of the relative amplitudes, following Shelly et al. (2016a). As the relative amplitudes are calculated from two waveforms recorded at the same station, there is no need to remove the instrument response.

This approach has proven to be more robust in the presence of relatively dissimilar waveforms than the method of Rubinstein and Ellsworth (2010), which assumes high correlation coefficients between all events in a family (e.g. $\geq 0.85$ ). In the Ngatamariki case, scattering and attenuation effects produce waveforms exhibiting lower degrees of similarity than typical repeating or near-repeating seismicity (e.g. along the San Andreas (Rubinstein and Ellsworth, 2010).

We used the GNS Science $M_{L}$ (as described in Section 3.2) to calibrate the relative moment calculations from the method above and produce $M_{L}$ estimates for matchedfilter detections. This was done by first converting the template local magnitudes to moment magnitudes using the scaling relationship

$$
M_{L}=0.97 M_{w}+0.14
$$

determined for locally detected, shallow New Zealand earthquakes (Ristau et al., 2016) and then converting to seismic moment using the equation Hanks and Kanamori, 1979):

$$
M_{w}=2 / 3 \log _{10} M_{0}-9
$$

Knowing the relative moment of the template event from the procedure outlined above, we then determined the relationship between the relative moments and actual moment which allowed us to convert relative moments to $M_{w}$ and then back to $M_{L}$ using Equation 3.4 .

\subsubsection{Focal mechanisms and stress inversion}

Focal solutions for selected events were calculated from P-arrival polarities using the Bayesian focal mechanism determination program of Walsh et al. (2009). Here, we present only focal mechanism solutions for the 86 events that occurred prior to plant startup in March 2013 and that had sufficiently high signal-to-noise ratio for the arrival polarities to be manually picked. These focal mechanisms were then used to invert for the stress parameters and the direction of maximum horizontal compressive stress $\left(\mathrm{S}_{\text {Hmax }}\right)$ within the northern and southern clusters separately, using the methodologies of Arnold and Townend (2007) and Lund and Townend (2007). 


\subsection{Results}

\subsubsection{Matched-filter detection}

Starting with the 1171 template events in the automatically detected catalog provided by GNS Science, we added 76,286 detections after matched filtering, creating a total catalog of 77,457 detections. At Ngatamariki, seismicity in both the GNS Science catalog and the matched-filter catalog falls into two spatial groups, which we refer to hereafter as the northern and southern clusters. We delineate the templates broadly by latitude whereby the northern cluster contains all templates located north of -38.55 degrees and the southern cluster contains all templates to the south (Figure 3.1). The northern cluster consists of 432 template events, which generated 25,482 detections. The southern cluster consists of 739 template events, which generated 50,804 detections. Two representative detections for a single template from the northern cluster are shown in Figure 3.2 .

\subsubsection{Event location}

We calculated preliminary locations for 41,114 events of the 77,457 total detections. Of the 3725 of these events for which we calculated magnitudes, we were able to relocate 2554. Given that events detected by the matched-filter method are, by design, similar to the template event that detected them, the locations of the detections should closely match the locations of the template events. Figures 3.3 and 3.11 show the detected and template events, respectively, plotted with depth and colored by their date of occurrence (blue for earlier in the dataset, pink for later).

In northern Ngatamariki, most events occur within a cloud of seismicity extending from $\sim 1500$ to 2500 meters below sea level ( $\mathrm{m} \mathrm{bsl}$ ). This cloud is spatially coincident with the dominant feedzones in well NM09 at between $\sim 1600-1800 \mathrm{~m}$ bsl (Figure 3.3). The injection rate during normal power plant operation is roughly $1100 \mathrm{t} / \mathrm{h}$ into NM09 and $\sim 300 \mathrm{t} / \mathrm{h}$ into NM08. During the stimulation of NM08, seismicity occurred within a narrow, NW-dipping band at $\sim 2200 \mathrm{~m}$ bsl. These events likely define the extent of a suitably-oriented fault that was activated during the treatment and which is discussed in more detail in Section 3.5.1.

In the south of the field, seismicity occurred in a cluster at depths of $\sim 2000$ $3000 \mathrm{~m}$ bsl, elongated in the NE-SW direction, and sub-parallel to the strike of the Aratiatia Fault Zone (Figure 3.1, 3.3). These depths coincide with the depths of the major feedzones in injection well NM06, not with the feedzones in NM10. Over the entire four-year dataset, seismicity in the southern injection zone migrates from SW to NE. We attribute this migration to a shift in injection strategy in this part of the field. Originally, injection was split equally between NM10 and NM06, but by 2015 all 


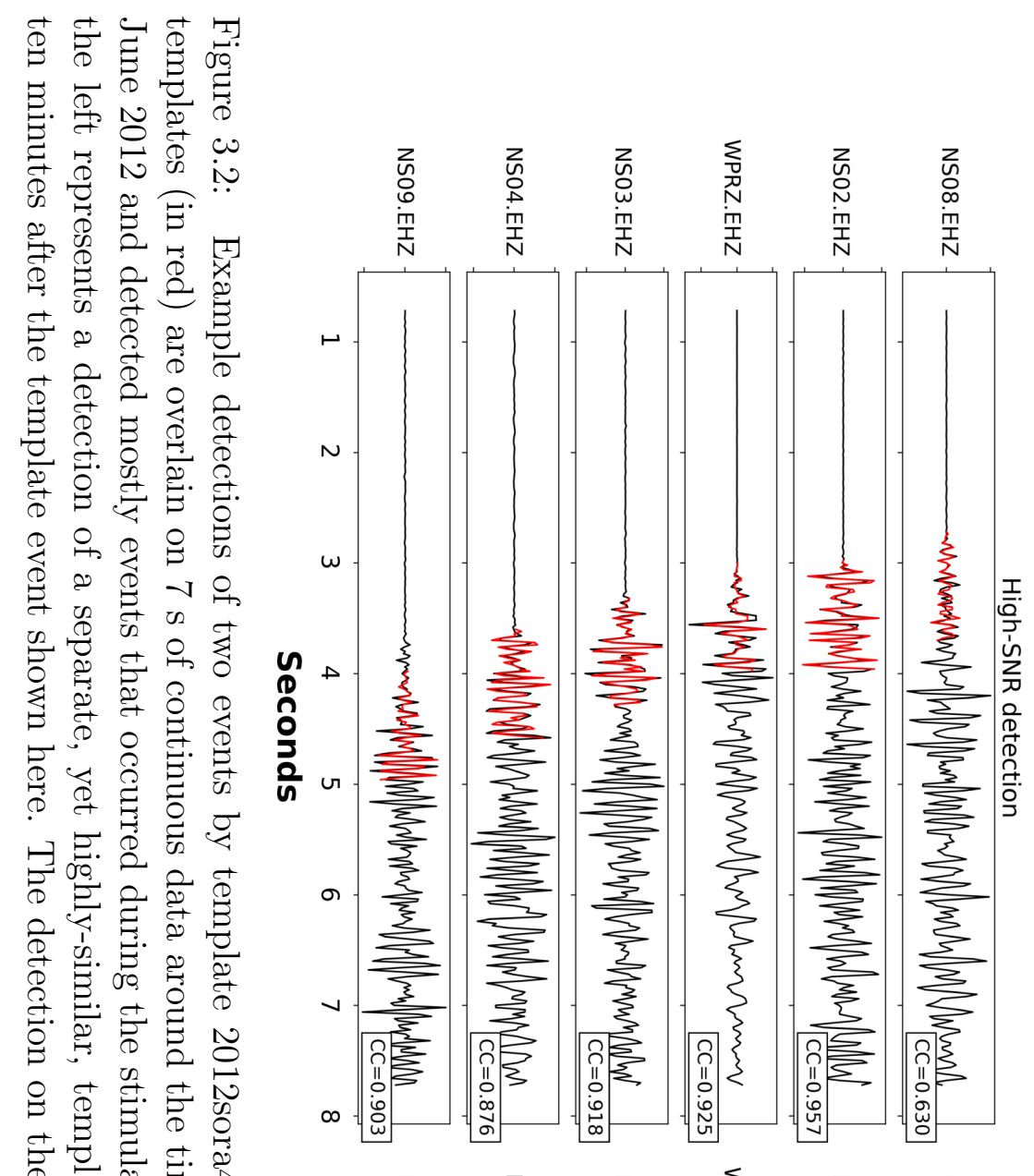

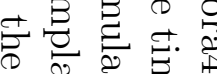

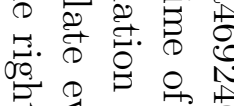

F

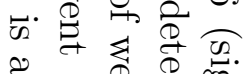

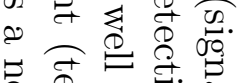

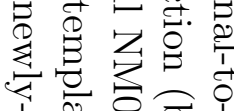

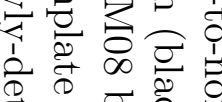

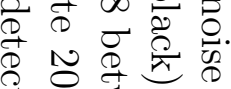

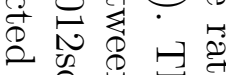

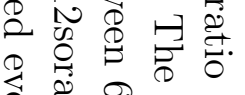

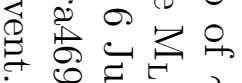

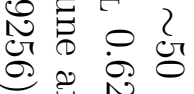
¿ हैं

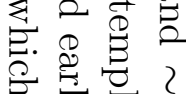
政

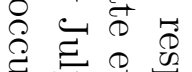
空 불붕

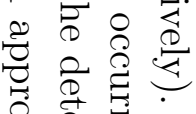
알. 울

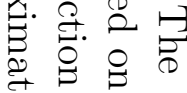
橧吕否

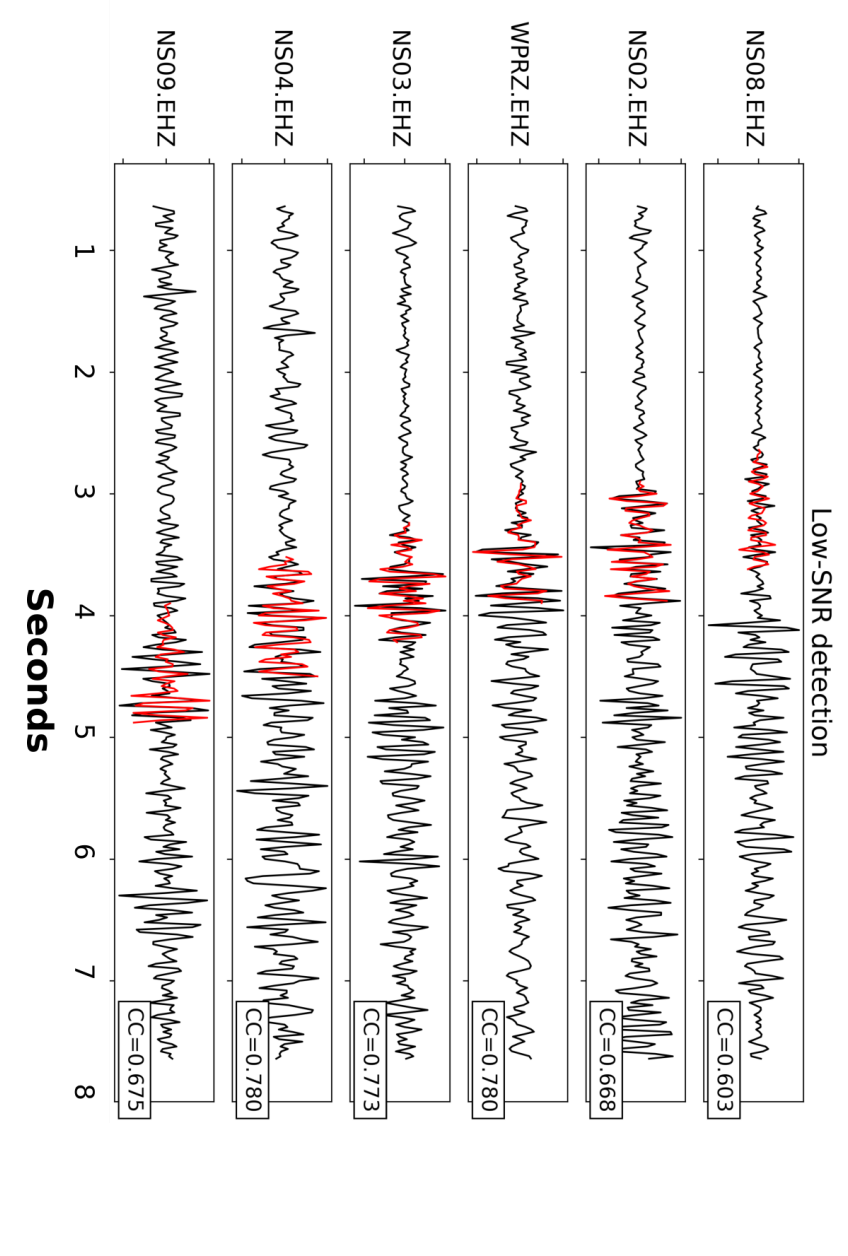


injection in the south was into NM06 due to rapid returns from NM10 to production well NM05 (Buscarlet et al., 2015).

The depth of seismicity in southern Ngatamariki is greater, on average, than in the north. In the south, hypocentral depths correspond to the depth of the Rotokawa Andesite and the lower Tahorakuri Formation (Chambefort et al., 2014) with shallower seismicity nearer to the production wells than the injection wells. In the north, below $\sim 2000 \mathrm{~m}$ bsl, the reservoir is dominated by the intrusive body Chambefort et al. 2014). The seismicity depth cutoff near both injection zones is likely related to the depth of permeable zones in the wells (Massiot et al., 2012; Halwa, 2013a. b). In the north, relatively little seismicity occurs within the bounds of the impermeable intrusive body and in the south most events are confined to the Rotokawa Andesite, which is known to be heavily fractured (Halwa, 2013b). The high temperature within the field likely also plays a role in controlling where microearthquakes are able to nucleate. Bottomhole temperatures for the northern injection wells reach $\sim 280^{\circ} \mathrm{C}$ and production well NM07 reaches almost $290^{\circ} \mathrm{C}$, approaching the brittle-ductile transition for quartzbearing rock (Scholz, 1988). In contrast, the maximum temperatures in the southern injection zone reach only $\sim 260^{\circ} \mathrm{C}$ and are located away from the main upflow in the field (between NM07 and NM08/09) (Chambefort et al., 2016). This temperature differential contribute to the difference in the depths of seismicity between the two injection zones, as the host rock should deform brittly to greater depths in the south than in the north.

\subsubsection{Magnitude}

We calculated magnitudes for 3725 of the 41,114 located events using the methodology outlined in Section 3.3.3. This large drop in the number of events is a result of the stringent correlation cut-off imposed ( $\geq 0.6$ at four or more stations, see Section 3.3.3), which ensures we retain only high-quality events. Figure 3.4 shows the frequencymagnitude distributions for both the GNS Science catalog (dotted lines), constituting the original template events, and the detected events for which we could calculate a local magnitude (solid lines). Here we show the catalog separated into northern and southern clusters. In both clusters, the additional matched-filter detections decrease the minimum magnitude in the catalog to less than zero.

We determine the $b$-values for the matched-filter catalogs for a magnitude of completeness $\left(M_{c}\right)$ calculated using the method of (Wiemer, 2000). . Notably, the inclusion of the matched-filter detections does not appreciably lower the magnitude of completeness of the catalogs here, in contrast to what has been shown elsewhere (e.g. Shelly et al., 2016a). In addition, the original catalogs (particularly in southern Ngatamariki) were not complete even at higher magnitudes, as suggested by the higher numbers of matched-filter detections for events above $M_{L} 2.0$ when compared to the GNS Science catalog. 


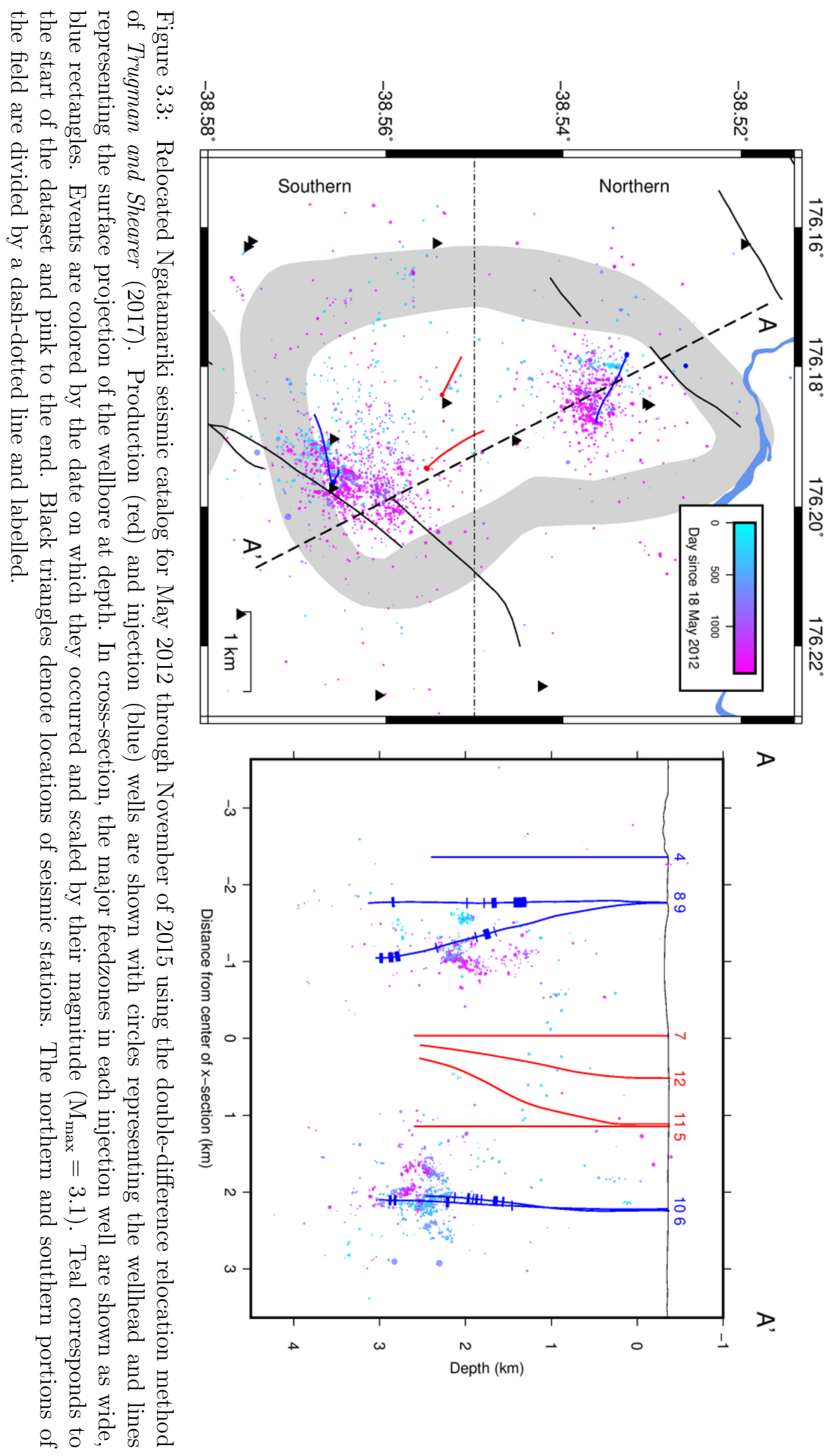


We suggest that the difference in the quantity of $\mathrm{M}_{L}>2$ events in southern Ngatamariki relative to the north reflects a difference in the sizes of fractures and faults that are hydraulically connected to nearby injection wells. The entire field is extensively fractured, as indicated by the available image logs of the injection wells Halwa, 2013a b; Massiot et al. 2012), but in the south, the active Aratiatia Fault Zone intersects injection wells NM06 and NM10 (Halwa, 2013b). The structures associated with this fault zone may be larger than those in the less-permeable northern portion of the reservoir where active structures are harder to identify (Massiot et al., 2012), allowing larger-magnitude events to nucleate. During injection operations elsewhere, higher $b$-values $(\sim 1.5-2.0)$ have been observed nearer the injection point where high pore fluid pressures can induce slip on fractures subject to low differential stress (e.g. Bachmann et al. 2012). Slip on these less-stressed fractures may be the explanation for higher $b$-values at injection sites and volcanic regions (Wiemer et al., 1998). This may also affect the $b$-values at Ngatamariki ( $b \sim 1.42$ for both clusters).

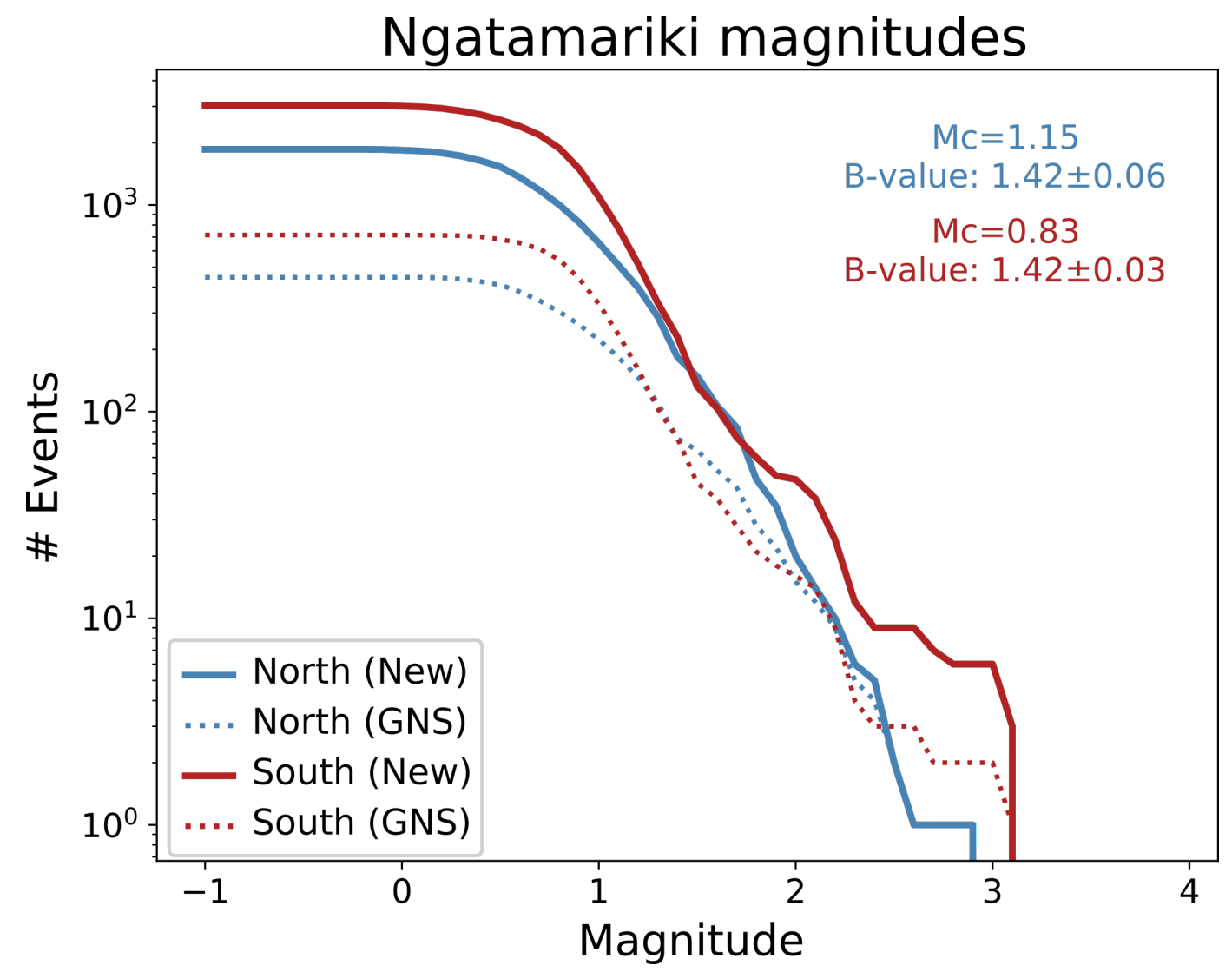

Figure 3.4: Cumulative frequency-magnitude distributions of the GNS Science catalog of templates (dotted lines) and the final matched-filter catalog (solid lines) for 20122015. Northern and southern Ngatamariki are shown in blue and red, respectively. The magnitude of completeness is calculated using the method of (Wiemer, 2000) . Calculated values of $\mathrm{M}_{c}$ and $b$ are noted in the top right. 


\subsection{Discussion}

Injection wells NM08, NM09 and NM10 underwent injection testing before the commissioning of the Ngatamariki power plant in 2013. These tests provide us the opportunity to study the seismic response to a number of isolated injections without contamination from concurrent injection from nearby wells. Below, we discuss each of these tests in detail and relate the characteristics of the accompanying seismicity to the injection parameters and geology in the respective part of the reservoir. Where possible, we calculate the well injectivity index and use it as a proxy for near-well reservoir permeability (Watson, 2013). Typically, injectivity varies as $t^{n}$ where $t$ is time since the start of injection and $n$ takes a value between 0.3 and 0.7 for most geothermal wells (Clearwater et al., 2015; Grant et al., 2013).

\subsubsection{Northern injection zone}

\section{NM08 stimulation}

Starting on 8 June, 2012, NM08 underwent a cold-water stimulation treatment, accepting $\sim 66,000 \mathrm{~m}^{3}$ of water over a period of approximately one month. This was the first injection test in the field and it triggered a sharp increase in the rate of microseismicity, which occurred predominantly in one cluster at the depth of the well's main permeable zone at $\sim 2000 \mathrm{~m}$ bsl (Figure 3.5). This sub-planar cluster strikes $\sim 192^{\circ}$ and dips $\sim 66^{\circ}$, centered on a depth of roughly $2000 \mathrm{~m}$ bsl. This is consistent with measurements of fracture orientation made from image logs, which suggest a dominant NE-SW strike for fractures intersecting the well and a dominant NW dip over this depth interval (Massiot et al., 2012). Flow rate, wellhead pressure and injectivity index are shown in Figure 3.5

Figure 3.5 shows the cumulative number of microseismic events during the NM08 stimulation, which included two phases of injection. The first, starting on 8 June, 2012 did not immediately trigger an increase in the rate of seismicity. Instead, there was a period of approximately ten days during which there were no detected events. We interpret this period to correspond to near-field pressurization of the reservoir (NF) followed by pressure breakthrough (BT) to a highly-permeable fracture zone (Figures 3.5 and 3.6). This period of testing involved a step-rate injection test in which the WHP response was observed for incremental changes in flow rate. Following this period, on 15 June (middle of BT, Figure 3.5 the injection was changed from a steady-flow to a steady-pressure regime in which flow rate was allowed to vary. This change also corresponded to a modest increase in both the injection rate and WHP. Roughly two days after this change, the rate of seismicity increased to between 5 and 12 events/day in the area of NM08 and this increased rate was maintained until the 
first phase of injection ended on 26 June (FZ1, Figure 3.5), accompanied by an abrupt halt to seismicity.

Phase two of the stimulation began on 1 July, 2012 (start of RP, Figure 3.5) at slightly higher flow rates than previously $(\sim 150 \mathrm{t} / \mathrm{h})$. Again, the rate of seismicity did not immediately increase once the second phase of injection began. There was a delay of approximately four days before the flow rate was increased to $\sim 175 \mathrm{t} / \mathrm{h}$ with an accompanying increase in WHP of approximately 0.5 MPa (FZ2, Figure 3.5). Following this increase, the rate of seismicity increased to 23 events per day (T5), slightly higher than the levels encountered during phase one. This activity ceased as soon as injection was halted on 9 July, bringing the total number of events during the stimulation to 122 . The maximum $M_{L}$ during the entire stimulation was 2.1.

Assuming that NM08 is hydraulically connected to a set of fractures, we expect seismicity to occur once an induced pressure-front has reached a critically-stressed subset of fractures in the local stress regime. The advance of the induced pressure front is controlled by the hydraulic diffusivity of the reservoir, which is closely linked to the reservoir permeability. This relationship has led other groups to develop seismicitybased reservoir characterization (SBRC) to infer hydraulic diffusivity from the location and occurrence time of induced seismicity (e.g. Shapiro et al., 2002; Shapiro and Dinske, 2009; Parotidis et al., 2004; Jeanne et al., 2015b). At Ngatamariki, and at most other naturally-fractured reservoirs, flow is controlled not by matrix permeability but by the spacing and permeability of fractures (Grant and Bixley, 2011). If we consider a large enough volume of the reservoir, relative to the fracture spacing, SBRC can be useful in estimating a bulk reservoir diffusivity from the occurrence of seismic events. However, if flow is concentrated in a small number of highly permeable fracture zones (highly anisotropic permeability), as is the case at Ngatamariki, SBRC performs poorly in describing reservoir properties. Nevertheless, we have fit a number of curves to the seismicity catalog for each of the injection scenarios detailed in this work (Figure 3.13).

The timing and location of seismicity during the stimulation of NM08 provoke a number of questions:

1. Why does seismicity lag behind the starts of injection by up to ten days?

2. Conversely, why does seismicity stop immediately following a halt of injection?

3. Why are the increase in injectivity index and seismicity poorly correlated in time?

Seismicity trails injection by ten days at the outset of the stimulation, and again by four days at the start of phase two (NF and RP, Figure 3.5). In the first tens of minutes following the start of injection, drilling-related degradation of near-well permeability (i.e. positive skin effects; Grant and Bixley, 2011) may have contributed to a delayed pressure response between the well and reservoir. However, as the seismic lag time was of the order of days, positive skin effects (normally on the order of hours) cannot 

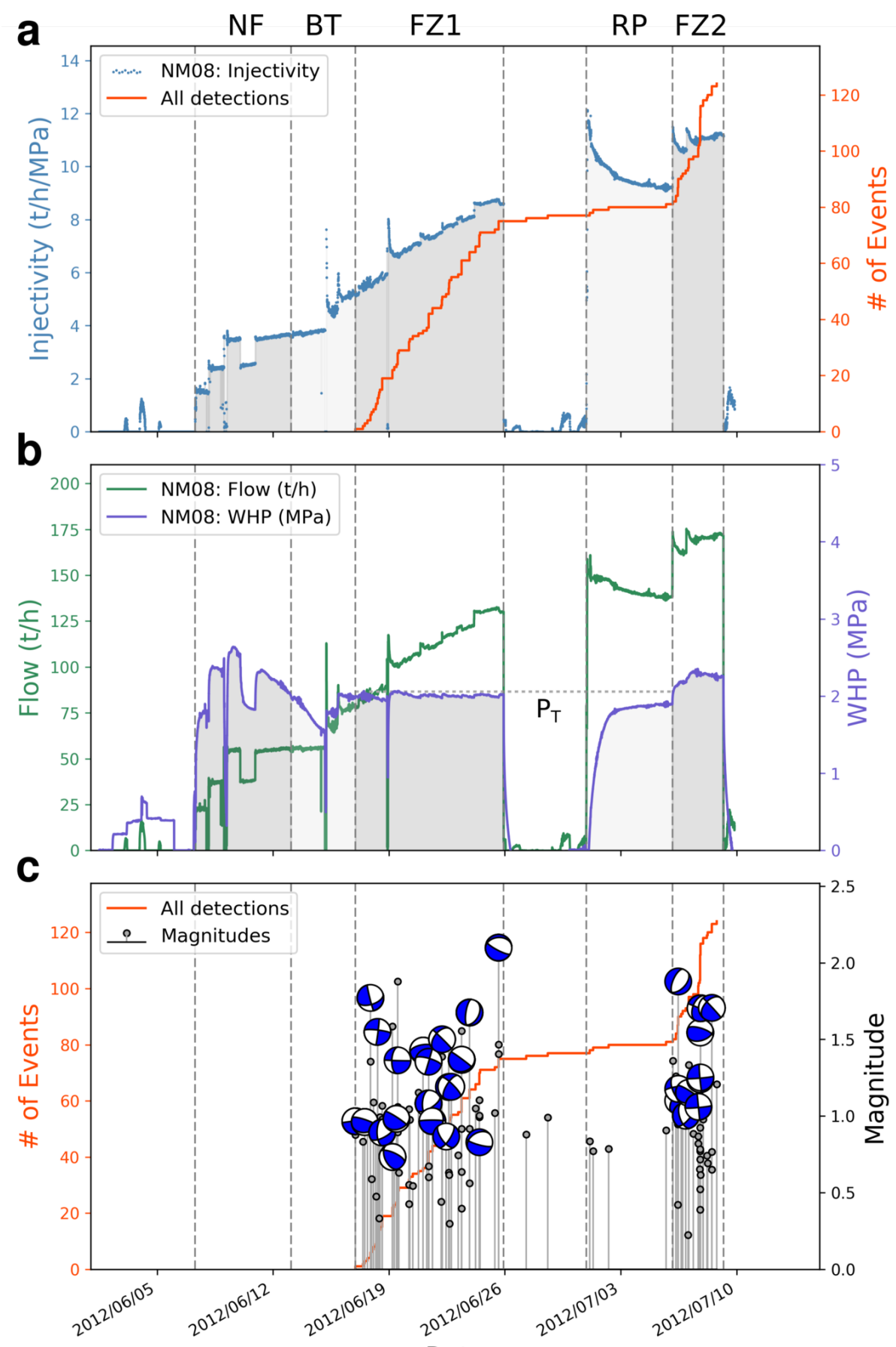

Figure 3.5: Summary of seismicity and injection parameters during the cold-water stimulation of NM08. a) Cumulative seismicity vs. injectivity index, b) wellhead pressure vs. flow rate and c) Local magnitudes and available focal mechanisms solutions for the stimulation of NM08. Labels at the top of the plot delineate the following periods of interest: NF: near-field pressurization of the fracture zones surrounding the dike swarm, BT: breakthrough of pressurization from the near-well reservoir to the seismically-active fracture zone, FZ1 and FZ2: fracture zone pressurization and associated seismicity, RP: renewed pressurization of the near-well reservoir. Once the fracture zone pore-fluid pressure exceeds the maximum induced pressure during FZ1 $\left(\mathrm{P}_{\mathrm{T}}\right)$, seismicity is again induced. These periods are detailed in the schematic in Figure 3.6. 
account for this observation $($ Horne, 1995$)$. We propose that the lag is due to geologic heterogeneity in the Tahorakuri volcaniclastic succession that hosts the reservoir, which defines where the permeable zones of the well are located, as well as the orientation of the fracture network.

The main permeable zone of NM08, which coincides with the depth of seismicity during stimulation, is defined by a series of mafic dikes or sills (Chambefort et al. 2014; Massiot et al., 2012) (Figure 3.6). Image log quality at this depth is poor, and the orientations of these dikes is poorly constrained (Massiot et al. 2 2012), but their emplacement orientation is likely to have been controlled by the prevailing stress field from 0.64-0.79 Ma (Chambefort et al. 2014 ) and related to the emplacement of the tonalite intrusive at the bottom of the well (Figure 3.6). If we assume that flow is concentrated within the damage zone surrounding the dikes and/or within the dikes' internal structures (such as cooling joints or flow bands Massiot et al., 2017), it may be that these structures are not optimally oriented for failure in the current stress regime. Although the prevailing tectonic environment during emplacement of the intrusive was similar to that of the present (i.e. active rifting Wilson and Rowland, 2016), highly active volcanism in the TVZ, including the subsequent formation of the adjacent Whakamaru caldera (0.35 Ma), would likely have modified the Ngatamariki stress state (Wilson et al., 1995).

We hypothesize that initial flow from NM08 was concentrated in these non-criticallystressed dike-related structures, and therefore did not trigger detectable seismicity (NF Figures 3.5, 3.6). In this case, the time lag in seismicity corresponds to a period of near-field pressurization during which NM08 was not hydraulically connected to the fracture zone defined by the hypocenters in Figure 3.7 (striking $192^{\circ}$, dipping $66^{\circ} \mathrm{NW}$ ). If we consider an alternative scenario, in which NM08 was always hydraulically connected to the fracture zone, then we would expect seismicity to have been triggered by the diffusion of the maximum observed WHP to the fracture zone (2.6 MPa during $\mathrm{NF}$, Figure 3.5). However, this scenario is unlikely in view of the immediate shutoff of seismicity when the well was shut-in, which would imply a hydraulic diffusivity an order of magnitude greater than that required by diffusion of the maximum WHP to the fracture zone. We discuss this in more detail below.

We infer that, during the near-field phase (NF), a small set of near-field fractures was pressurized, with a maximum measured wellhead pressure of $2.6 \mathrm{MPa}$ (the highest WHP measured at Ngatamariki to date). Seismicity was induced in the fracture zone (roughly $200 \mathrm{~m}$ from NM08) during a period of constant 2.0 MPa WHP (FZ1, Figure 3.5). The hydraulic connection to the fracture zone would need to have been established while the WHP was below this $\sim 2.0 \mathrm{MPa}$ threshold, otherwise seismicity would have been induced earlier. The degree to which the fracture zone was critically stressed may also explain a part of the time lag during what we call 'breakthrough' (BT, Figure 3.5), 
as it may have taken time for the pressure perturbation to reach the value, $\Delta P_{\text {crit }}$, at which it induced slip.

A common observation during injection operations worldwide is post-injection, persistent seismicity (e.g. Fehler et al., 1998, Rutledge and Phillips, 2003; Shapiro et al., 2002). Many of the largest injection-induced events have occurred after shut-in of the injection wells in question (e.g. Mukuhira et al., 2017, at Basel). At Ngatamariki this was not the case, as near-well seismicity ceased with the halt in injection for both phases of the NM08 stimulation (end of FZ1 and FZ2, Figure 3.5). Assuming an unbounded reservoir, the relaxation of the shut-in-induced pressure perturbation is also a diffusive process (termed the 'back-front' of seismicity by Parotidis et al. (2004)). In the NM08 case, an immediate cessation of seismicity on shut-in would imply nearly infinite reservoir permeability between the well and the seismically active zone. This is incompatible with flow through the reservoir rock matrix, which has a calculated in-situ permeability of $6 \times 10^{-18} \mathrm{~m}^{2}$ Cant et al. 2018), but could occur if the effective reservoir permeability is controlled by fractures. If we conceptualize the set of fractures connecting NM08 to the active fracture zone as a series of pipes with effectively infinite permeability, then a pressure perturbation applied at one end produces a near-instant pressure response on scales of 100s of meters (the so called 'water hammer' effect; Ghidaoui et al. 2005). The fracture network at Ngatamariki would have sufficient permeability to transmit these pressure perturbations rapidly to distances of 100 s of meters, far enough to reach the seismically active zone. Once a drop in pore fluid pressure has propagated to the critically stressed fracture zone ( $200 \mathrm{~m}$ from NM08), seismicity should cease. We suggest that in order for the initial time lag and eventual rapid halt in seismicity during NM08 stimulation to coexist the hydraulic conductivity between NM08 and the seismically active fracture zone must have been established during the injection (during BT, Figure 3.5).

Injectivity gain and seismic slip during the stimulation of NM08 are not well correlated. During phase one of injection (NF, BT and FZ1), injectivity increased significantly $\left(n=0.6-0.7\right.$, where injectivity increases as $t^{n}$ for geothermal wells Clearwater et al., 2015; Grant et al., 2013), indicating that near-well permeability was increasing. However, during near-field pressurization (NF), no seismicity was detected, suggesting that the initial permeability enhancement resulted from aseismic processes as has been observed elsewhere (Cornet, 2016, and references therein). The rate of injectivity increase was also insensitive to the eventual onset of seismicity on 17 June (FZ1, Figure 3.5). Guglielmi et al. (2015) measured three-dimensional fault displacement (with micrometer precision) and estimated permeability enhancement during the initial stage of a decameter-scale injection experiment, showing that most of the estimated permeability gain was a result of fault-normal displacement and not shear slip Guglielmi et al. 2015). While the temperature contrast between the injection fluid and reservoir was negligible for their experiment, we propose that a similar period of fracture- 
normal, aseismic displacement occurred during the drilling and subsequent stimulation of NM08. The large temperature contrast between injected fluid and reservoir rock at Ngatamariki (up to $\sim 226^{\circ} \mathrm{C}$ ) served to encourage fracture-normal displacement as a result of thermal contraction of the fracture walls. As suggested by Stephens and Voight (1982), the thermally-induced circumferential stress at the wellbore is (e.g. Zoback, 2010):

$$
\Delta \sigma_{T}=-\frac{\alpha_{L} E \Delta T}{1-\nu}
$$

with $\alpha_{L}$ being the coefficient of thermal expansion of reservoir rock, $E$ Young's modulus, $\Delta T$ the temperature difference between reservoir rock and injectate and $\nu$ Poisson's ratio. We assume $\alpha_{L}=1 \times 10^{-5} \mathrm{~K}^{-1}$ (Bauer and Handin, 1983), $E=20 \times 10^{9} \mathrm{~Pa}$ (Cant et al. 2018), $\nu=0.25$ and a temperature difference of $204^{\circ} \mathrm{C}$ (interpreted from pressure-temperature spinner data at feedzone depth during stimulation. This yields a thermal stress at the wellbore of approximately $-54 \mathrm{MPa}$. Understanding the direction and rate of flow is necessary to interpret how such thermal stresses would affect the effective stresses acting on a set of fractures. As we do not know the predominant flow direction, especially within and around the dike swarm, we cannot comment on the effect on the criticality of the fractures. However, the lack of seismicity suggests that such cooling effects may have stabilized the fracture network as was modeled by Jeanne et al. (2015a).

The thermal diffusivity of the reservoir matrix is typically orders of magnitude lower than the effective hydraulic diffusivity of the reservoir $\left(\mathrm{mm}^{2} / \mathrm{s}\right.$ and $\mathrm{m}^{2} / \mathrm{s}$, respectively) (Kanamori et al., 1968; Shapiro et al., 2002). Nevertheless, these thermal effects likely dominate the increase in well permeability at NM08. This model of thermal-expansiondriven stimulation has been proposed by Grant et al. (2013) and Siega et al. (2014), who tested it against a number of geothermal injectivity datasets in New Zealand and elsewhere. Self-propping of slipping fractures, which is associated with seismic slip, is also known to increase fracture permeability (e.g. Lee and Cho, 2002), and this process undoubtedly influences the permeability of the seismically-active fracture zone. However, the injectivity increase as measured at the well appears to be far more sensitive to other processes, which we suggest include near-well thermal contraction of the fracture network walls and pore-pressure-induced normal displacement.

Phase two of NM08 stimulation was also accompanied by a time lag (this time of four days) prior to the response of seismicity. In this case, the lag corresponds to the time until the previous highest pore-pressure perturbation $\left(\mathrm{P}_{T}\right.$, Figure 3.5) was exceeded. This behavior is often referred to as the Kaiser effect (Holcomb, 1993). Assuming that hydraulic connectivity from NM08 to the active fracture zone was not established until the 'breakthrough' period (BT, Figures 3.5, 3.6), the highest pressure perturbation to which the fracture zone was subjected during the period of seismic slip 
corresponded to a $\overline{\mathrm{WHP}}$ of roughly $2 \mathrm{MPa}$. As soon as WHP exceeded this $2 \mathrm{MPa}$ threshold during phase two, seismicity restarted. This reinforces the interpretation of a high permeability fracture network within $200 \mathrm{~m}$ of NM08 following the near-field $(\mathrm{NF})$ and breakthrough (BT) of phase one (Figure 3.5.

Recent research by (Cappa et al., 2019) shows that fluid injection can encourage rate-strengthening behavior of a fracture, implying that seismic slip is triggered by poroelastic stress transfer away from the pressurized zone. Considering these findings, an alternative explanation for the triggering of seismicity at NM08 does not require a hydraulic connection between the well and the seismically-active zone. Instead, seismic slip may have been purely the product of Coulomb stress transfer onto the active fracture zone by aseismic displacements near the wellbore. As described above, nearwellbore aseismic displacements were likely induced by a combination of thermoelastic contraction of the rock matrix and pore-pressure increase in the feedzones.

\section{NM09 injection testing}

Injection well NM09, in the north of the field, underwent two periods of injection prior to plant startup (Figure 3.12), accepting $\sim 56,000 \mathrm{~m}^{3}$ of water between 13 December, 2012 and 4 January, 2013 and $\sim 60,000 \mathrm{~m}^{3}$ of geothermal brine between 12 February and 6 March, 2013. The second of these tests used geothermal fluid injected directly from the plant $\left(\sim 90^{\circ} \mathrm{C}\right)$, and not river water $\left(\sim 10-20^{\circ} \mathrm{C}\right)$ as was injected at NM08, meaning that the degree of thermal contraction of the near-wellbore fracture walls was likely less than during the first portion of the NM09 test or at NM08. If we perform the same calculation for circumferential (hoop) stress as done previously for NM08, but with a $\Delta T$ of $120^{\circ} \mathrm{C}$, instead of $204^{\circ}$, we get a thermally-induced stress of $-32 \mathrm{MPa}$, or $\sim 60 \%$ of the stress induced by cold-water stimulation. Only 11 seismic events were detected within the reservoir during both phases of NM09 stimulation combined. Given that NM08 and NM09 were drilled in the same part of the reservoir, the difference in seismic response is surprising. However, Clearwater et al. (2015) pointed out that there are major structural differences between NM09 and NM08, despite their proximity. NM08 was drilled directly into the low-permeability intrusive body in this section of the field, whereas NM09 is deviated and was completed in the intrusive's highly-permeable damage zone. Importantly, image logs reveal that NM09 intersects a number of highly-permeable fault zones at depths of $\sim 1850-2300 \mathrm{~m}$ bsl (Halwa, 2013a), which were not intersected by NM08. During reservoir tracer testing, it was also shown that NM09 is better-connected to the production wells than NM08 (Buscarlet et al., 2015). This implies a degree of compartmentalization in the northern reservoir, with the structure delineated by the NM08 seismicity possibly acting as a cross-strike flow barrier (Buscarlet et al., 2015) (Figure 3.7). 

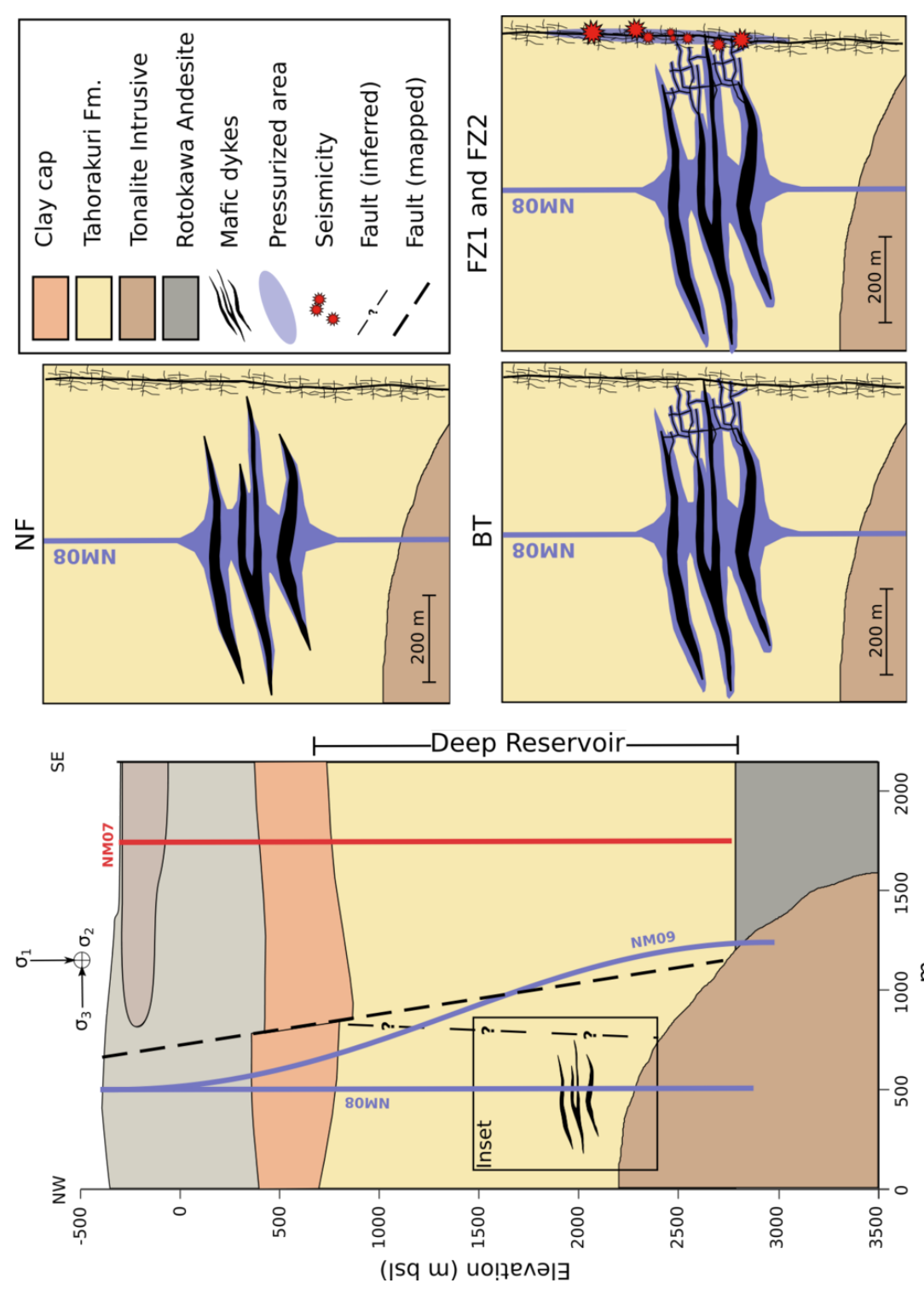
The high-permeability feedzones at NM09 inhibit pressure buildup in the reservoir during injection. During the injection tests, WHP at NM09 never exceeded 0.1 MPa and was often zero, implying that fluid entered the reservoir under its own weight, thereby decreasing the likelihood of inducing seismicity through pore-pressure diffusion. However, injectivity increased in the absence of seismic slip ( $\mathrm{n} \sim 0.4-0.6)$ Clearwater et al., 2015). As at NM08, this observation indicates that permeability was enhanced through undetected (small) seismic slip, aseismic slip/opening of permeable zones connected to the wellbore, or both. It also reveals an even stronger decoupling of injectivity and near-well seismicity than at NM08, with the observed gain likely a result of thermoelastic stresses near the wellbore, especially during cold water injection in phase one.

\subsubsection{Southern injection zone}

\section{NM10 drilling losses and injection testing}

Well NM06 was drilled and tested in the southern injection zone many years prior to our study. As a result, drilling and well testing in southern Ngatamariki during this study period was limited to injection well NM10. Injection began once drilling had reached the depth of the deep (andesite) reservoir and drilling-fluid losses occurred (at depths $>2000 \mathrm{~m} \mathrm{bsl}$ ). This was followed by a formal injection/stimulation test once drilling had been completed. The microseismic response to the drilling losses was larger than the response during the actual injection test (Figures 3.8 and 3.9). At the end of drilling, approximately $57,000 \mathrm{~m}^{3}$ of drilling fluid (largely water) had been lost to the formation, a comparable volume to that in the other injection tests analyzed and described above. Data on fluid flow during drilling are sampled less frequently than the flow rate data during injection tests, but daily records of drilling losses correlate well with seismicity in southern Ngatamariki as shown in Figure 3.8a. NM10 drilling reached $1700 \mathrm{~m}$ bsl on 6 July 2012 and significant fluid losses were incurred from 13 July ( 2100 m bsl).

Seismicity began as the drilling reached the depth of a major fault zone identified in the NM10 image logs (there are six identified faults between 2100 and $2300 \mathrm{~m}$ bsl, within the Rotokawa Andesite; Halwa, 2013b) and continued for the remainder of the drilling (Figure 3.8c). These SE-dipping structures, visible in image logs (Halwa, 2013b), are likely associated with the NE-SW-striking Aratiatia Fault Zone (Figures 3.1, 3.3, 3.7). Seismicity increased in short, 1-2 day-long bursts with the majority of events occurring after fluid losses had exceeded $25 \mathrm{t} / \mathrm{h}$. The lag between the start of fluid losses and the seismicity induced during NM10 drilling is roughly three days, three times shorter than at NM08. This lag may simply be due to the time needed to pressurize the fault zones, which dominate flow from NM10. However, aseismic opening of the fault zones 

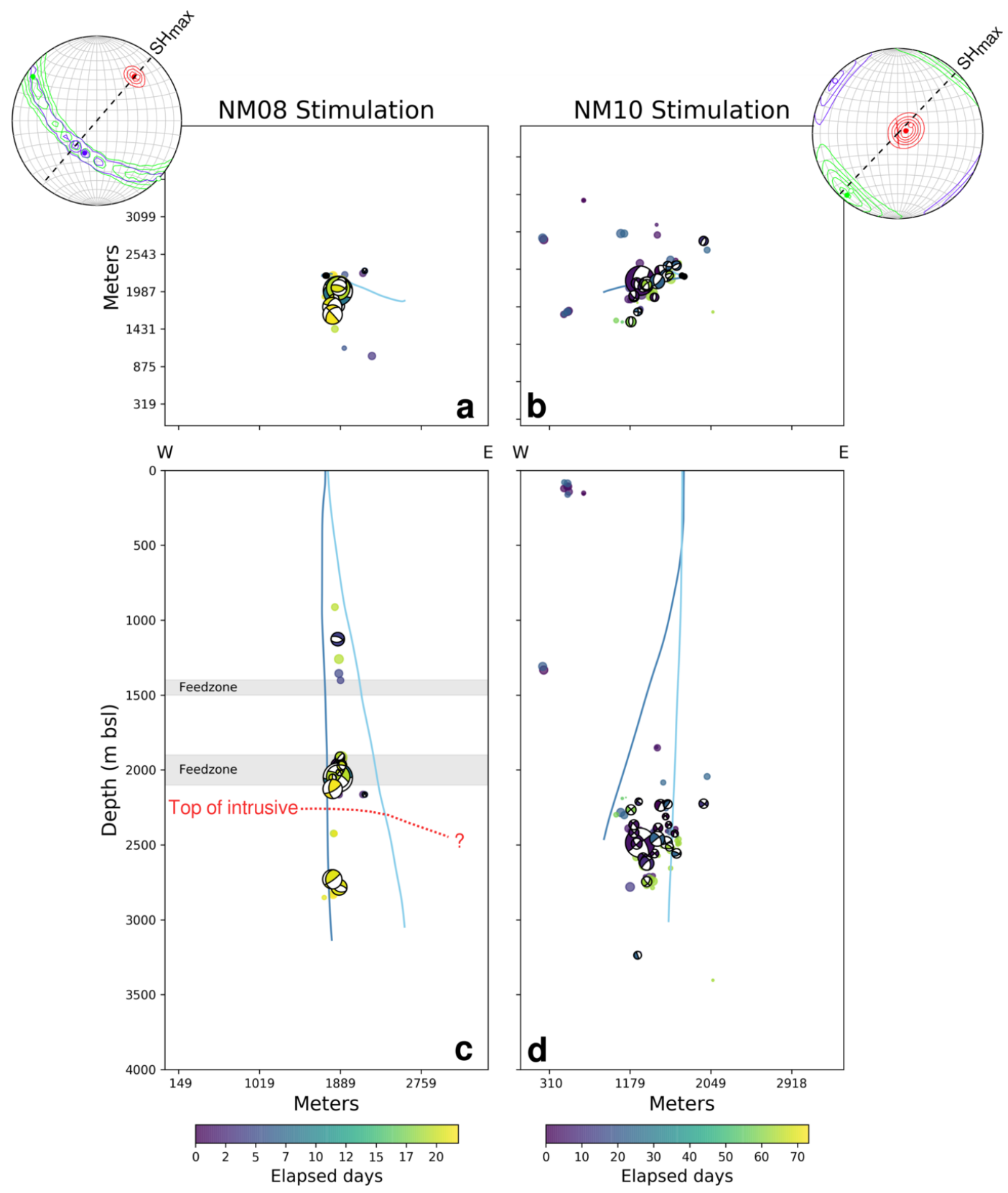

Figure 3.7: P-wave first-motion-derived focal mechanisms for template events which occurred during various phases of injection testing at Ngatamariki. Dark blue mechanisms in a and c occurred during stimulation of NM08, in the northern injection zone. Green mechanisms occurred during NM10 drilling losses and injection testing (panels $\mathrm{b}$ and $\mathrm{d}$; southern zone). The symbols plotted in cross-sections $\mathrm{c}$ and $\mathrm{d}$ have been reprojected to reflect the different viewpoint (from the south). The inset figures in $\mathrm{a}$ and $\mathrm{b}$ show stress inversion results (lower hemisphere) from the focal mechanisms presented in northern and southern Ngatamariki, respectively, with red contours representing the probability density of the direction of $\sigma_{1}$, green representing $\sigma_{2}$ and blue representing $\sigma_{3}$. The black dashed line indicates the direction of $\mathrm{S}_{\text {Hmax }}$. In panel c, the red dashed line indicates the top of the intrusive sequence in northern Ngatamariki. As the extent of this unit is only constrained by core from wells NM04 (northeast of panel a), NM08 and NM09, its shape, volume and orientation are unknown. 
via pore-pressure increase and thermal contraction of the fracture walls, as observed by Guglielmi et al. (2015) and Cappa et al. (2019), may also have played a part. We would expect these processes to be accompanied by a corresponding increase in injectivity, but there are no pressure data during the drilling to confirm this. The seismicity occurred $>700 \mathrm{~m}$ deeper than the main feedzones in NM10 (Figure 3.3), and closer to injection well NM06, which was shut-in during this period. This may indicate that fluid flowed down-dip along the fault zone to critically stressed points away from the well. The maximum magnitude during drilling at NM10 was 2.1, comparable to the stimulation at NM08 for a similar injected volume. This may indicate that the pressurized zones during NM08 stimulation and NM10 drilling were similar in volume, thereby affecting fractures and faults of similar size. However, as southern Ngatamariki exhibits a greater number of $\mathrm{M}_{L}>2$ events than in the north when the entire four-year catalog is taken into account, we suggest that much larger structures exist in the south than the north, which were only activated once high-volume injection began in 2013. As at NM08, seismicity ceased after the end of drilling, implying that the permeability of the fracture zone is considerable.

There were no drilling or injection activities in southern Ngatamariki between the drilling of NM10 and the planned injection test that occurred on 1-23 September, 2012. There was also no detected seismicity during this period. Roughly $73,000 \mathrm{~m}^{3}$ of fluid was injected during the test at flow rates of between 100 and $200 \mathrm{t} / \mathrm{h}$ and downhole pressures of 7.5-9.4 MPa (Figure 3.9). As mentioned above, the unintended losses that occurred during drilling are considered the start of injection in southern Ngatamariki. Therefore, during drilling, NM10 may have undergone much of the increase in injectivity that otherwise would have occurred during the later injection test. It is possible that slip during drilling relieved most of the accumulated stress on the most critically stressed portions of the fault zone, making failure on these patches less likely during the injection test. This view is supported by the relative lack of seismicity during most of the test (Figure 3.9). Seismicity, while not absent, occurred for the first two weeks of the test at a rate of roughly one event per day, and injectivity increased only slightly after the first step-rate change in flow from $\sim 100$ to $150 \mathrm{t} / \mathrm{h}$. However, the rate of seismicity jumped dramatically (with nearly 40 events on 17 September alone) once injection was increased from $\sim 150 \mathrm{t} / \mathrm{h}$ to $\sim 200 \mathrm{t} / \mathrm{h}$. We do not know the corresponding increase in downhole pressure for this step-rate change because the pressure monitoring tubing was repositioned at that time. However, because the flow rate of 200 $\mathrm{t} / \mathrm{h}$ is significantly higher than was measured during either drilling or testing prior to this time, we can reasonably assume that the downhole pressure would have increased beyond any previously-recorded level at NM10, and therefore would be expected to induce seismicity (again due to the 'Kaiser effect'; Holcomb, 1993). The resulting increase in the rate of seismicity occurred within two hours of the increase in flow rate and at event-feedzone distances of up to $800 \mathrm{~m}$ (Figure $3.9 \mathrm{a}$ and $3.9 \mathrm{~b}$ ). As during FZ2 

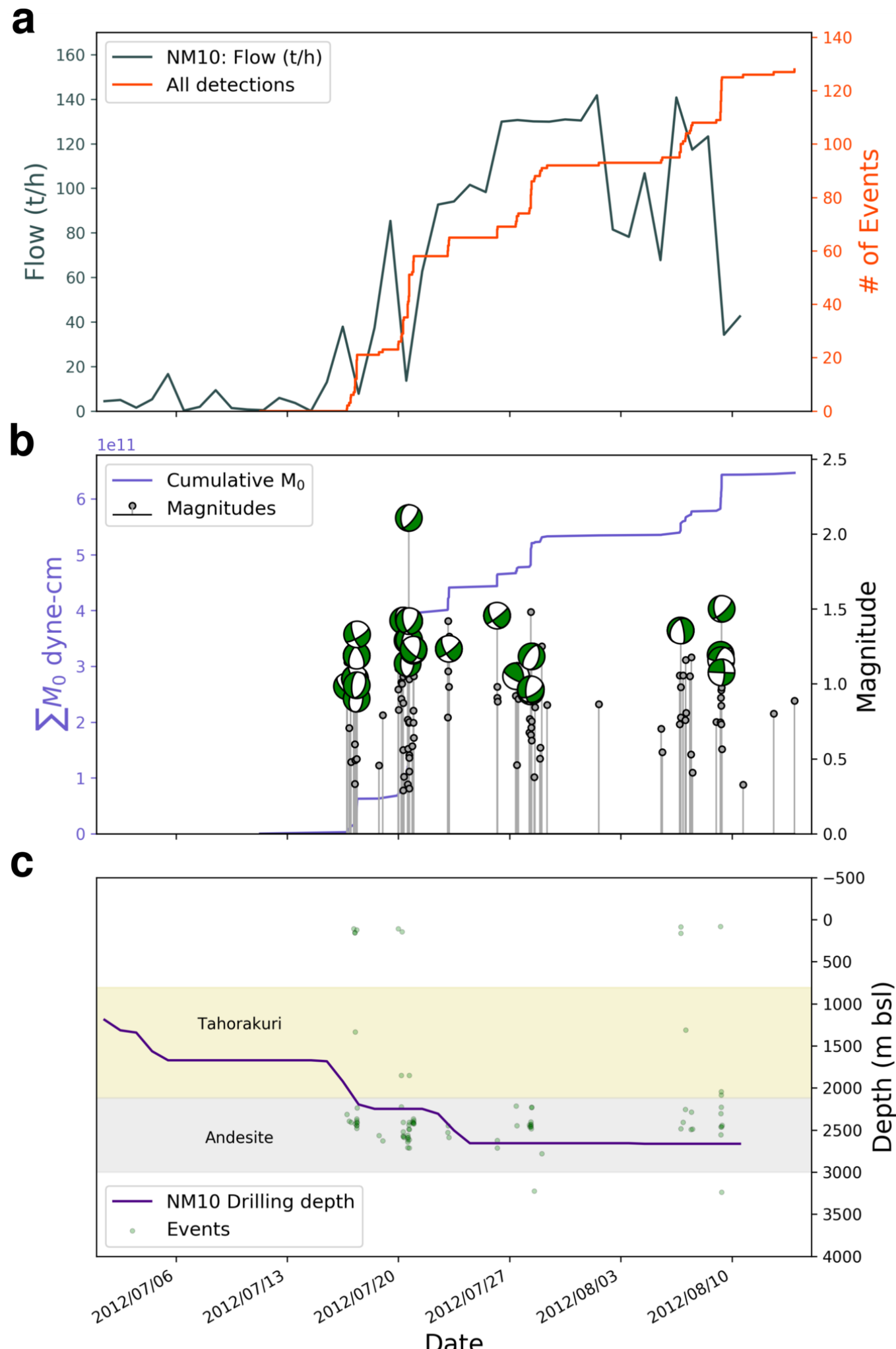

Figure 3.8: a) Flow (t/h) during fluid losses and cumulative seismicity vs time, b) cumulative seismic moment with event local magnitudes and available focal mechanisms during drilling of NM10 and c) drilling depth with depth of seismic events. The two main geological units within the southern reservoir, the Tahorakuri volcaniclastics and Rotokawa Andesite, are depicted in c) for context. 
at NM08, inducing events at such distances so rapidly requires that pressure change be concentrated along a small number of highly-permeable fracture zones, which is what we expect in NM10 on the basis of image logs, or that triggering is purely the result of poroelastic stress transfer onto non-pressurized portions of the fracture zone. Once flow rate had subsided to below $200 \mathrm{t} / \mathrm{h}$, seismicity ceased, suggesting rapid progression of the back-front to the seismically active zone as was observed at NM08. The maximum magnitude during the injection testing of NM10 was 1.4, lower than during NM10 drilling, possibly as a result of the stress acting on the fracture zone having been relived during drilling fluid losses.

\subsubsection{Focal mechanisms and stress}

Figure 3.7 shows the focal mechanisms calculated for microearthquakes that occurred during the three injection tests described above, and the corresponding stress inversion results for northern and southern Ngatamariki prior to plant startup. The events that occurred during drilling and injection testing of NM10 (Figure 3.7b and d) delineate a structure associated with the Aratiatia Fault Zone, as mentioned above. The majority of the 28 focal mechanisms we calculated for southern Ngatamariki during this time period show predominantly normal faulting, with most fault planes striking N-S or NESW. This result agrees well with what is known about the extensional regional stress regime and the local faults in the central TVZ, which are typically oriented NE-SW (Massiot et al., 2015b). Acoustic- and resistivity-based image logs for NM10 confirm this preferred NE-SW fracture orientation at reservoir depths with predominantly SE dips (Halwa, 2013b). As mentioned in Sections 3.1.3 and 3.4.2, rapid injection returns during a tracer test conducted in January 2014 also indicate that a highly permeable flow pathway connects NM10 to production well NM05 (Buscarlet et al., 2015). The locations of the events induced during NM10 drilling and injection testing are likely related to fluid flow along this structure.

Stress inversion results for southern Ngatamariki (inset, Figure 3.7b) show $\sigma_{1}$ to be subvertical and the direction of maximum horizontal compressive stress $\left(S_{H \max }\right)$ to be NE-SW, consistent with previous findings for the central TVZ Townend et al. 2012) as well as measurements of drilling-induced tensile failure (DITF) in NM10 which indicate an $S_{\text {Hmax }}$ azimuth of $210^{\circ}($ Halwa, 2013b). This result is also consistent with DITF measurements from the Rotokawa geothermal field, $5 \mathrm{~km}$ to the south McNamara et al., 2015).

The 58 blue mechanisms in Figure $3.7 \mathrm{a}$ and c correspond to events that occurred during the stimulation of NM08 and exhibit a greater variety of faulting kinematics than in the south. Many of these mechanisms show primarily reverse or oblique strikeslip movement with at least one nodal plane striking NW-SE, which agrees with the orientation of the plane defined by the hypocenters of the events (strike $192^{\circ}$, dip 

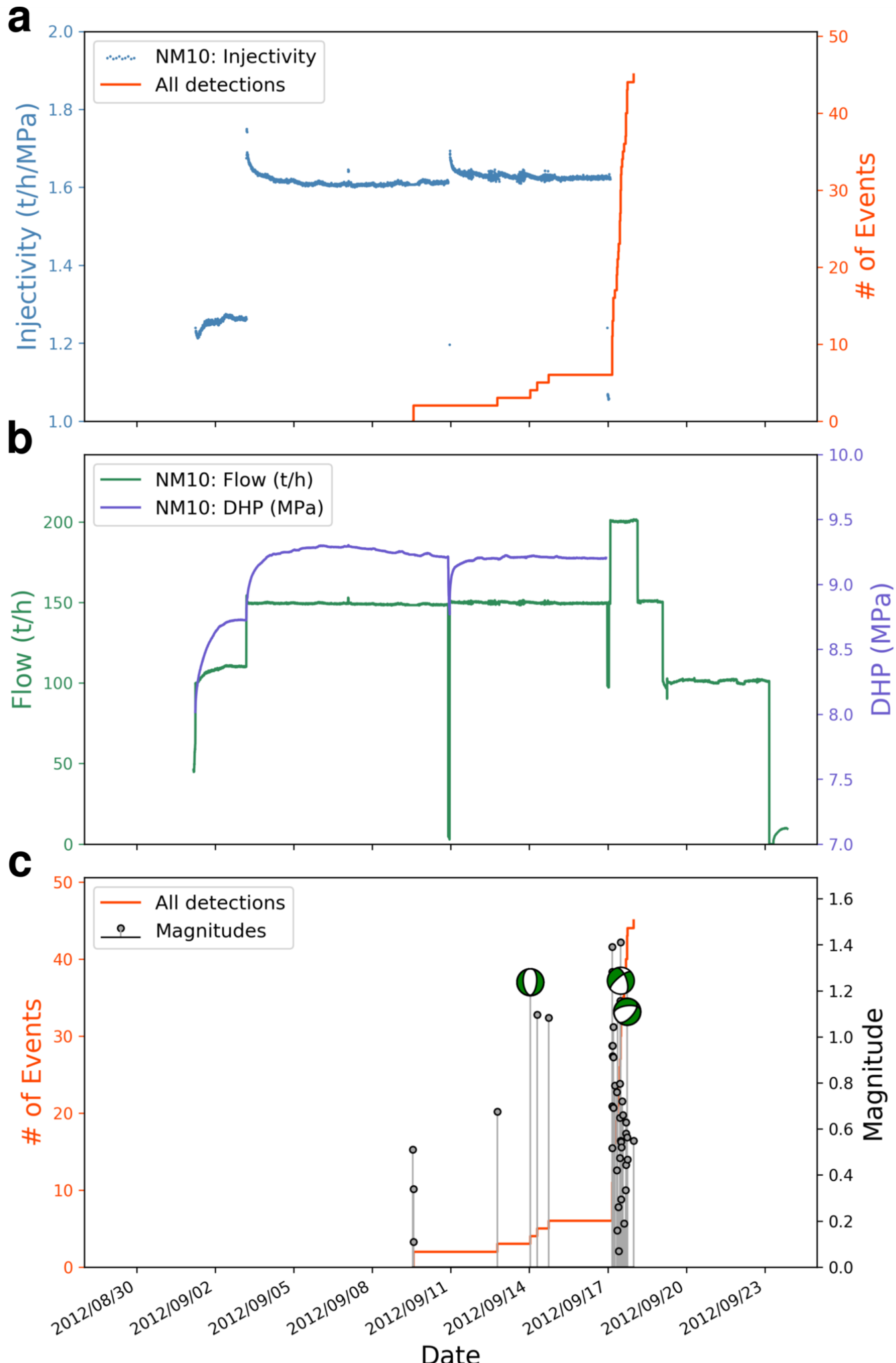

Figure 3.9: a) NM10 injection test II vs. cumulative seismicity in southern Ngatamariki, b) Flow rate and downhole pressure. DHP ends on 17 Sept as the measurement tubing was traversed to a greater depth (from $1228 \mathrm{~m}$ to $2050 \mathrm{~m}$ ). c) Cumulative seismicity in red with the magnitude and available focal mechanism solutions as stems. 
$\sim 66^{\circ}$ ). The remaining mechanisms show normal faulting with a variety of strikes. The occurrence of compressional faulting is rare within the central TVZ and may be related to stress field rotation during the emplacement of the intrusive body and mafic dikes.

Stress inversion results for northern Ngatamariki differ from those in the south (insets in Figure $3.7 \mathrm{a}$ and b). While the direction of $S_{\text {Hmax }}$ is unchanged throughout the reservoir (NE-SW), $\sigma_{1}$ is dipping NE at approximately $30^{\circ}$ in the north and $\sigma_{2}$ and $\sigma_{3}$ define a girdle. It is possible that the stress state in this section of the reservoir was already rotated into an orientation suitable for reverse faulting by the emplacement of the tonalite intrusive body and dikes. Massiot et al. (2012) showed that the in-situ horizontal stress field rotates counter-clockwise by $28^{\circ}$ near the contact between the Tahorakuri and the intrusive body (below the main permeable zone and dikes), relative to the stress field above, which may indicate an intrusive-related effect on the stress state.

What is not known is the effect of injection-related pressure buildup and thermoelastic stresses on the stress in the reservoir (Figure 3.10). We have no DHP measurements during stimulation of NM08. However, wellhead pressure reached 2.6 MPa during the near-field period of stimulation (NF, Figure 3.5). Figure 3.10a shows the destabilizing effect of pore pressure increase on the reservoir stress state, with the increase in $\mathrm{P}_{f}$ exaggerated for visibility. We infer that the fracture zone defined by the seismicity during NM08 stimulation is nearly-critically-stressed within the reservoir stress regime and would require only small increases in pore pressure or Coulomb stress to induce slip (blue and red circles, Figure 3.10a).

Circumferential stresses reached roughly $-54 \mathrm{MPa}$ at the wellbore (at $2000 \mathrm{~m} \mathrm{bsl}$ ), but it is difficult to determine how far such thermoelastic effects would propagate from the well as the propagation of the thermal front is dependent upon the flow pathways and flow rate through the reservoir. During a stimulation operation at the naturallyfractured Geysers geothermal field, Jeanne et al. (2015a) modeled a similar thermal front propagating $>100 \mathrm{~m}$ in the span of two months. For a reservoir where fluid flow is fracture dominated, the corresponding cooling effect depends on the dominant direction of flow relative to the principal stress axes, as illustrated in Figure $3.10 \mathrm{~b}$ (Jeanne et al., 2015a). This is because the rock matrix is cooled conductively only within $<1 \mathrm{~m}$ along the normal to a fracture plane, whereas it is cooled for many tens of meters along the strike of a fracture, leading to an anisotropic volume of thermal cooling (Simone et al., 2013, Jeanne et al. 2015a). Initial, gravity-driven (downward) flow of cool fluid at NM08 would preferentially decrease $\sigma_{1}$ and stabilize the near-well fracture network (yellow Mohr's circle, Figure 3.10b). This may have contributed to the lack of seismicity during the NF and BT periods of NM08 stimulation (Figures 3.5 and 3.6. As fluid begins to spread laterally from the well, the cooling effect preferentially lowers the horizontal stress that is best aligned with the direction of flow. At Ngatamariki, fluid flow parallel to $\sigma_{3}$ (NW-SE) would act to destabilize the fracture network (pink 
Mohr's circle, Figure 3.10b), including the structure along which seismicity occurred, which is represented as a colored circle for the various stress fields illustrated in Figure 3.10 .

As the Ngatamariki reservoir is structurally similar to that of The Geysers, in that they are both naturally-fractured, high-temperature fields, it is possible that a thermoelastic front could have reached the location of seismicity during stimulation of NM08 and therefore influenced the stress state in the fracture zone. Also at The Geysers, Martínez-Garzón et al. (2013) observed temporal changes in their focal-mechanismderived stress tensors related to various fluid injection operations, including the injection operation modeled by Jeanne et al. (2015a), although this contrasts with the findings of Boyle and Zoback (2014) who found that operations at The Geysers had little effect on the reservoir stress state. We cannot rule out the possibility that the deviation of the stress field from the regional trend in northern Ngatamariki is injectionrelated, although we think it unlikely. As discussed above, it is also possible that the stress state was already rotated in the northern injection zone (relative to the regional, purely-normal regime) prior to injection.

\subsection{Conclusions}

Ngatamariki constitutes an important case study of isolated injection into a previously little-modified reservoir. In this paper we present a nearly four-year catalog of microearthquakes at the Ngatamariki geothermal field spanning periods of initial field development, well drilling and plant startup. Individual tests at each well were isolated from one another in both time and space, which has allowed us to observe the response of microseismicity to injection at individual wells. Seismicity occurs in two spatial clusters centered on the northern and southern injection zones. In the north, stimulation of well NM08 induced more than 120 events in one month, while injection testing of nearby well NM09 generated only 11 events in nearly three months. In southern Ngatamariki, drilling and injection testing of NM10 generated nearly 200 events over an interval of roughly three months. The difference between the frequency-magnitude distributions of the northern and southern clusters provides a clear example of magnitude dependence on the characteristics of the local fracture network. In the north, the fractures that are hydraulically connected to wells NM08 and NM09 are smaller than those in the south, where fluid is injected into a large, active fault zone. As a result, larger events are able to nucleate in the southern injection zone than in the north.

Focal mechanisms calculated for events that occurred during these injections show different faulting regimes in the northern and southern halves of Ngatamariki. During the stimulation of NM08, in the northern part of the field, focal mechanisms exhibited a wide range of faulting kinematics, most having at least one nodal plane consistent with 

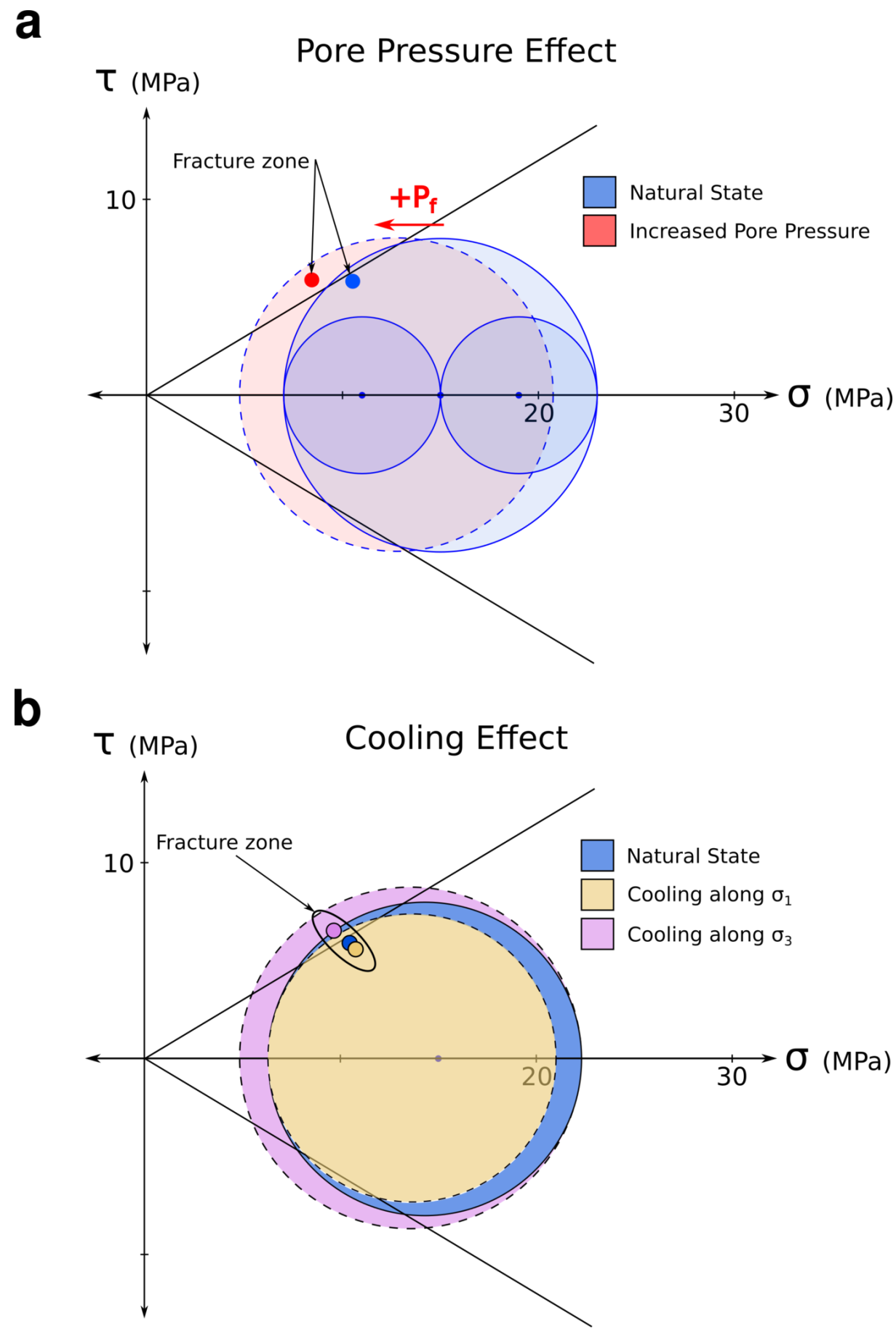

Figure 3.10: Schematic Mohr's circles calculated for a depth of $2000 \mathrm{~m}$ bsl illustrating the effect of a) pore pressure increase and b) thermal cooling of the fracture zone near well NM08 (strike: $191^{\circ}$, dip: $66^{\circ}$ ). The natural state magnitude for $\sigma_{1}$ was determined by integrating density over depth using density values from well cuttings and core taken from NM08 ( $\sim 5 \mathrm{MPa}$ at $2000 \mathrm{~ms}$ ). The magnitude of $\sigma_{3}$ was extrapolated from leak-off tests conducted in Rotokawa ( $\sim 29 \mathrm{MPa})$ (Davidson et al., 2012). Reservoir pressure is approximately $22 \mathrm{MPa}$ at $2000 \mathrm{~m}$ bsl. The failure envelopes represent cohesionless, preexisiting fractures with a coefficient of friction of 0.6. Circles in the figure represent the position of the plane defined by the hypocenters of seismicity during NM08 stimulation within each stress field. Plots are adapted from the output of MohrPlotter (Allmendinger et al., 2011). 
the NNE-SSW trend in hypocenter locations and a stress state with $\sigma_{1}$ dipping $30^{\circ}$. In contrast, NE-SW-striking normal faulting mechanisms make up the bulk of events occurring in the south. We interpret this contrast to be related to the presence of the tonalite intrusive body encountered at the bottom of the wells in northern Ngatamariki, which likely modified the local stress field in such a way that reverse and strike-slip faulting could occur in close proximity. However, as has been modeled and inferred for The Geysers geothermal field, another high-temperature, naturally-fractured reservoir (Martínez-Garzón et al., 2014, Jeanne et al., 2015a), injection-induced stress tensor changes are plausible at Ngatamariki.

Fluid injection-induced slip on nearby, suitably oriented fractures is commonly assumed to be the main mechanism responsible for well stimulation, In the Ngatamariki case, however, induced seismicity occurs independently of near-well permeability gain, suggesting that seismic slip and permeability gained through self-propping play a subsidiary role in well stimulation. During the cold-water stimulation test at NM08, injectivity increased rapidly from the start of injection with no apparent sensitivity to the onset of seismicity ten days later. During injection at NM09, the well was also stimulated but was accompanied by only 11 detected seismic events despite two separate injections totaling $>100,000 \mathrm{~m}^{3}$ of fluid. In the south, interpretation of the cold-water stimulation of well NM10 was complicated by fluid losses during drilling, which induced nearly 140 events but for which no injectivity data exist.

This apparent decoupling of induced seismicity and near-well permeability suggests that slow, aseismic processes dominate wellstimulation at Ngatamariki, specifically, but also at other high-temperature, high-permeability geothermal reservoirs where porepressure increase and thermoelastic stresses may lead to fracture opening and stable sliding (Cappa et al. 2019). Other recent studies have raised the possibility that permeability and seismicity need not be related for cases of induced seismicity (e.g. Guglielmi et al., 2015; Riffault et al., 2018) and the dataset we present here contributes to the documentation and understanding of this discrepancy.

The degree of decoupling may be a product of the combined high-permeability and high-temperature of the Ngatamariki reservoir. Lower-temperature resources will not exhibit similar degrees of thermal stimulation and lower-permeability reservoirs are subject to higher pore-pressure perturbations. However, (Cappa et al., 2019) have shown that, even for isothermal reservoirs, elevated pore pressures encourage stable slip. If permeability enhancement and seismicity are decoupled, as we have shown for Ngatamariki, it may be possible to design injection operations in similar settings elsewhere in order to achieve the desired permeability gain while limiting the number and magnitude of induced seismic events. 


\subsection{Acknowledgments}

We thank the Rotokawa Joint Venture (Tauhara North No. 2 Trust \& Mercury NZ Limited) for the funding to conduct this research and for allowing us access to the data and permission to publish our findings. We also wish to acknowledge the contribution of high-performance computing facilities to the results of this research. New Zealand's national facilities are provided by the NZ eScience Infrastructure (NeSI) and funded jointly by NeSI's collaborator institutions and the Ministry of Business, Innovation \& Employment's Research Infrastructure program (https://www.nesi.org.nz). The analysis reported here made use of the ObsPy seismic processing toolbox (The ObsPy Development Team, 2016), and the matched-filter detection was conducted using the EQcorrscan package (Chamberlain et al., 2017a; Chamberlain and Hopp, 2016) which can be freely downloaded and installed via $\mathrm{PyPI}$ or Anaconda on all major platforms. The documentation is hosted at ReadTheDocs.

The final version of the Ngatamariki earthquake catalog can be found at DOI 10.17605/OSF.IO/C2M6U along with access to the github repository of all scripts used in this work.

\subsection{Appendices}

The bulk of this section contains supplemental materials submitted to G-cubed with the manuscript above. Certain figures and text from the original supplemental materials file were included in the Data and methodology in Chapter 2 and have therefore been removed from this section to avoid redundancy. Newly-added material is shown in Section 3.8.4 In Section 3.8.4, we compare the injected volume and maximum moment release of the stimulations at NM08 and NM10 with other injection scenarios worldwide.

\subsubsection{Template locations}

Figure 3.11 shows the locations of the template events used in the matched-filter detection procedure at Ngatamariki. These events were relocated using the GrowClust algorithm of Trugman and Shearer (2017). As is required by the matched-filter method, the location of the template events is similar to the locations of the detected events shown in Figure 3.1.

\subsubsection{NM09 stimulation and testing}

Figure 3.12, summarizes the injection parameters and accompanying seismicity from December 2012 to March 2013, during which time there were two separate injection 


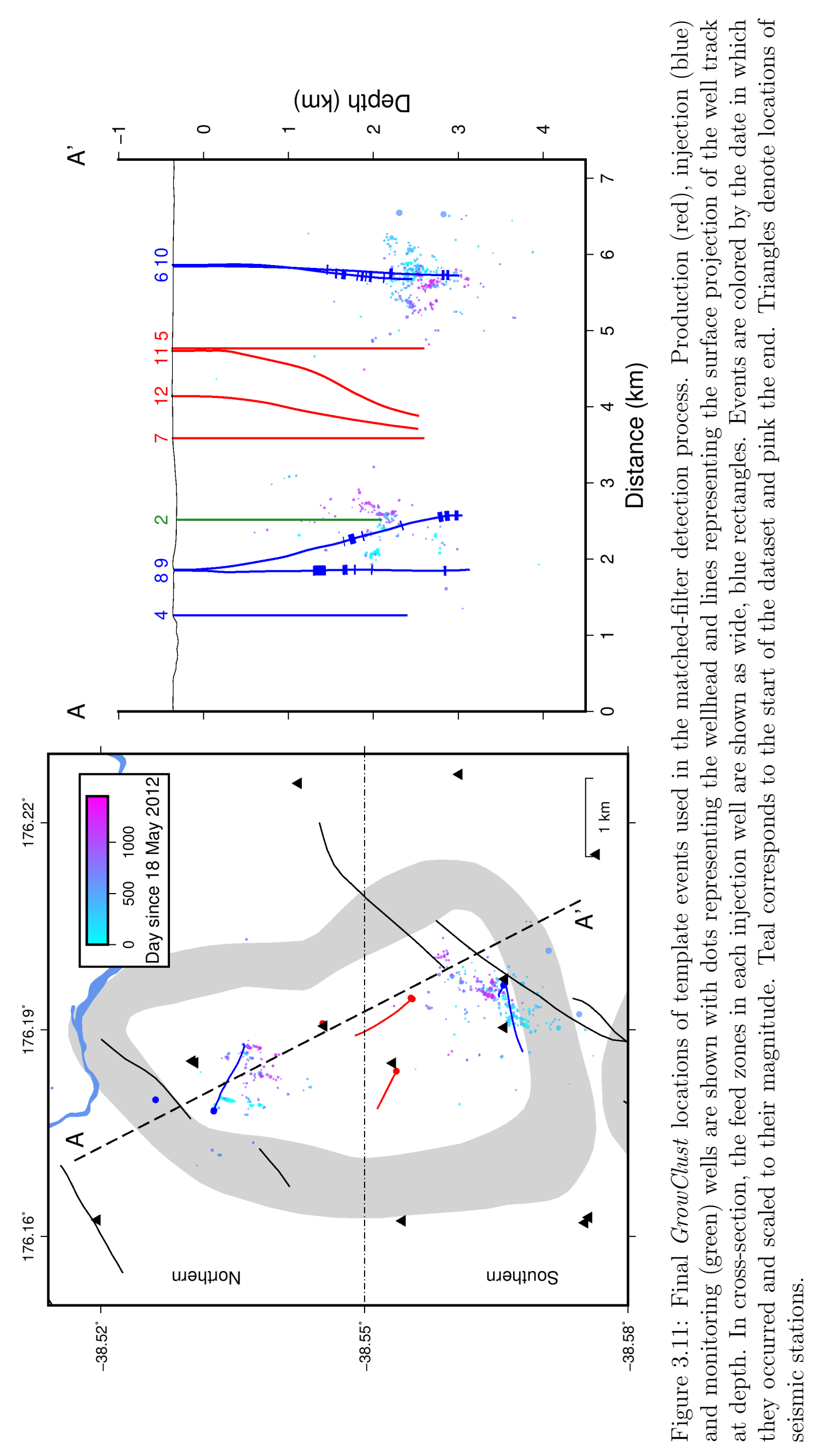


operations at NM09. Relative to the other injection scenarios covered in this work, these operations were relatively aseismic (a total of 11 events over this period). We have included this figure to support the claim of aseismicity we made in the above text.
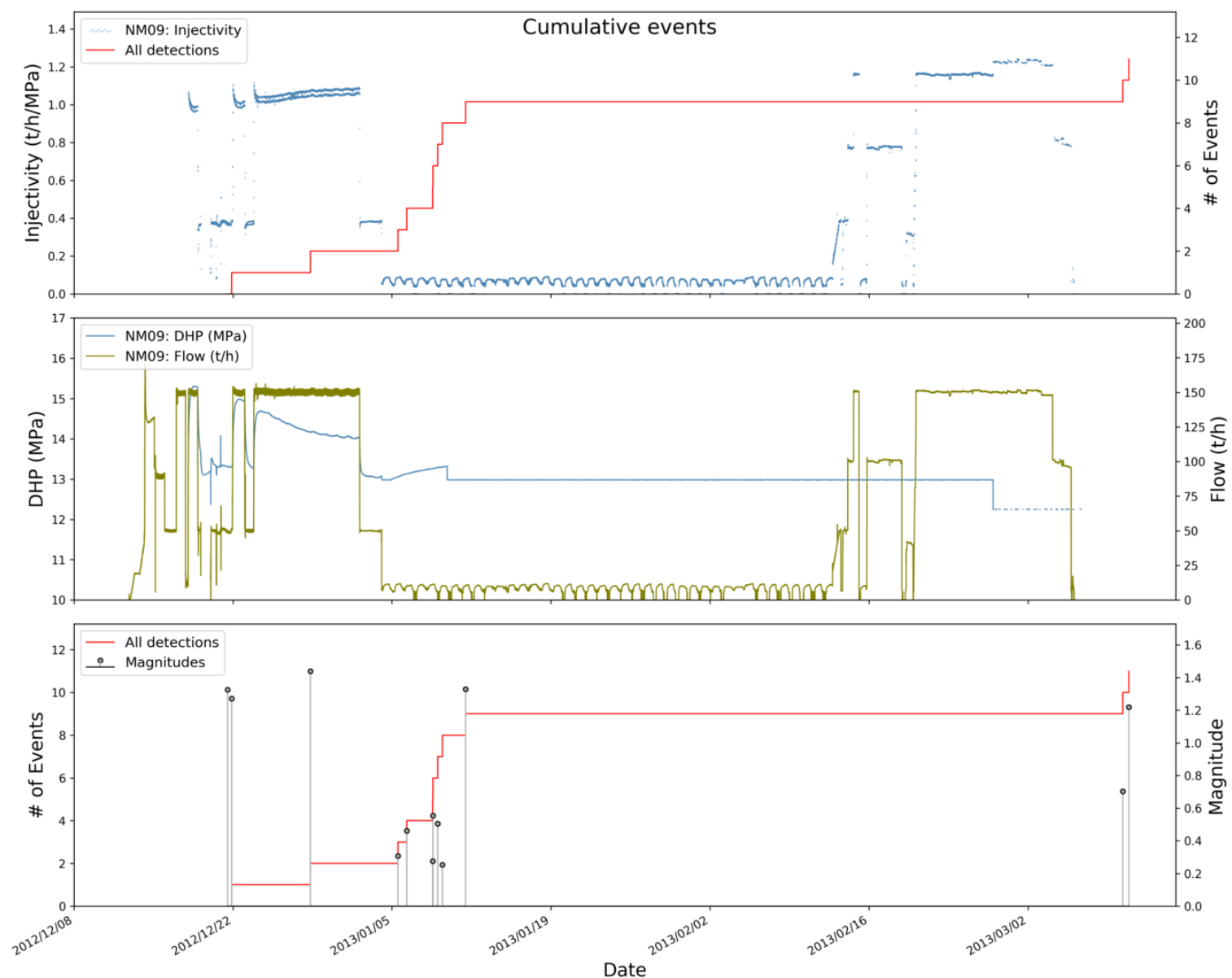

Figure 3.12: Seismicity and injection parameters during both phases of well testing at NM09. The top panel shows injectivity calculated from DHP (blue) and cumulative seismicity (red). The middle plot shows DHP (blue) with flow rate (green) and the bottom panel shows cumulative seismicity (red) with the magnitude of each event as a stem.

\subsubsection{Seismicity-based reservoir characterization}

We have fitted a number of 'triggering front' curves to the seismicity data for the NM08 stimulation. While it is possible to fit these data with isotropic triggering fronts corresponding to a range of hydraulic diffusivity and pressure perturbation, these models are not necessary to explain the spatial patterns in the data. Flow in the Ngatamariki reservoir is highly anisotropic and seismicity is tightly-clustered in both space and time. In addition, most seismicity occurs at least $200 \mathrm{~m}$ away from the wellbores, perhaps as a result of stabilization of the fracture network due to near-well 
thermoelastic stress effects, which was suggested to explain a similar occurrence at The Geysers by Jeanne et al. (2014).

We fitted three 'triggering front' curves for two simplified scenarios at NM08. The first is the case of isotropic permeability surrounding the main feedzone of NM08, in which case the triggering front can be approximated by a sphere of seismic activity centered around the feedzone (Figure 3.13, top panel). However, this is likely not the case at NM08 where we expect permeability to be highly anisotropic. Considering our proposed scenario where a pressure breakthrough occurs between the near-well reservoir and the fracture zone, we also model pressure diffusion from this hypothetical 'breakthrough' point, which we approximate as the center of the cluster of seismicity (Figure 3.13 , bottom panel). This point could conceptually be thought of as a hydraulic 'leak' from the near-well reservoir into the fracture zone. In this case, pressure diffusion would migrate radially from this breakthrough point along the plane of the fracture zone.

We fit the curves by eye with the aim of incorporating all events within the uppermost quartile of the daily event distance distribution (blue boxplots in Figure 3.13). It is notable that all values of diffusivity, $\mathrm{D}$, are $\leq 0.1$, and are therefore similar to estimated values for reservoirs that we would expect to be much less permeable than Ngatamariki. For example, Shapiro et al. (2002) show $\mathrm{D}=0.05 \mathrm{~m}^{2} / \mathrm{sec}$ at the Soultz EGS site in France and Shapiro et al. (2006) estimate $\mathrm{D}=0.01 \mathrm{~m}^{2} / \mathrm{sec}$ for the KTB borehole in Germany. Where the SBRC technique has been applied to naturally-fractured systems, such as during the Prati-32 EGS project at The Geysers geothermal field, estimated diffusivity values have been much larger $(\mathrm{D}=0.18-0.2)($ Jeanne et al., 2014). The permeability of the Ngatamariki reservoir, as a whole, is more similar to The Geysers case. However, it is possible that the low values estimated here for the stimulation of NM08 reflect the actual hydraulic properties of the northern part of the reservoir, which is known to be of lower permeability than elsewhere at Ngatamariki Chambefort et al., 2014).

The fitting of isotropic pore-pressure diffusion curves to the NM08 data (for either scenario), is not convincing and is not necessary to explain the location of the seismicity. It is likely that highly anisotropic permeability at the $<1 \mathrm{~km}$ scale in Ngatamariki, invalidates such simple modeling of the migration of fluid and pressure.

\subsubsection{Maximum magnitude}

McGarr (2014) derived a relationship between the cumulative volume of injected fluid and the maximum seismic moment of induced seismicity:

$$
M_{0}(\max )=G \Delta V
$$



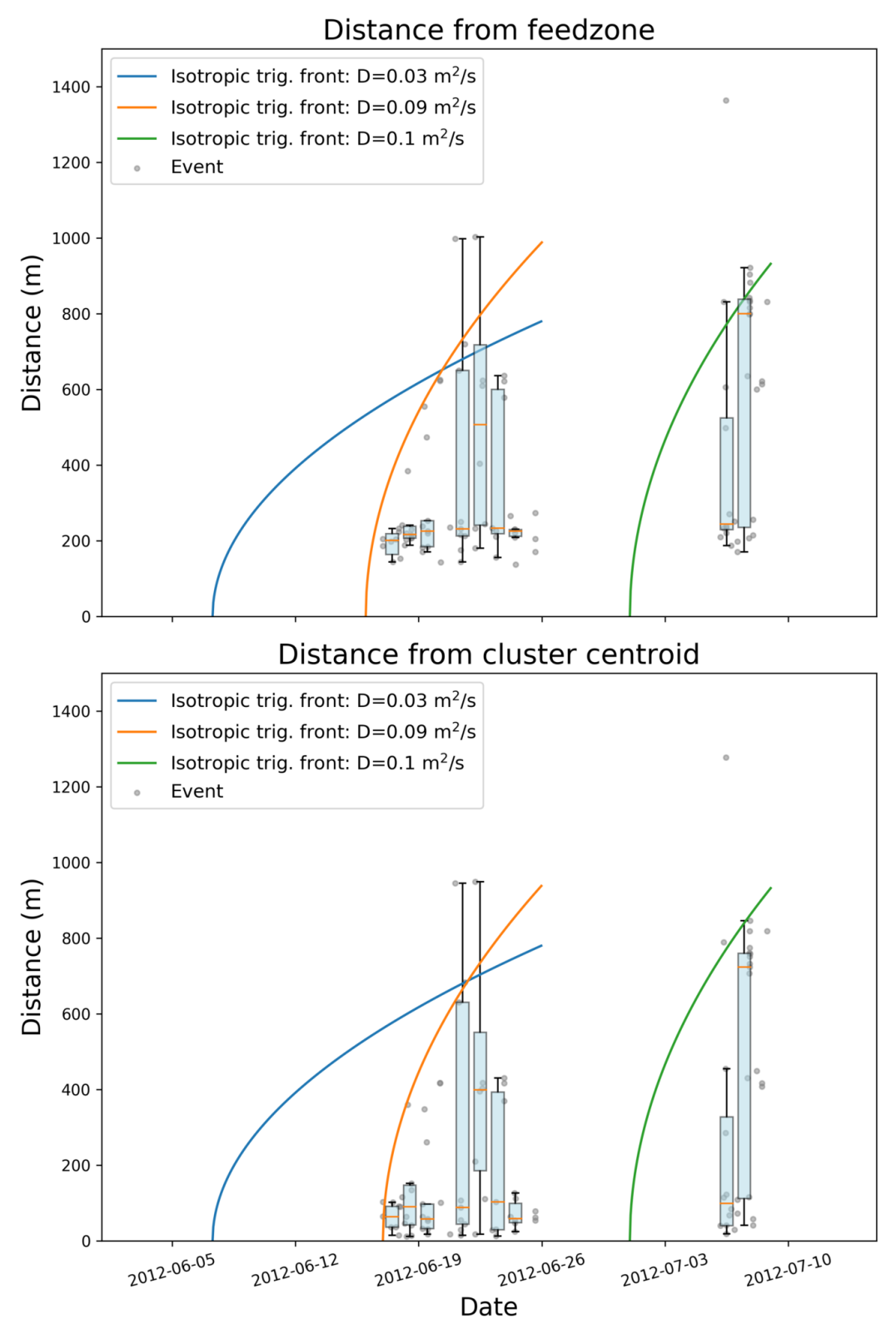

Figure 3.13: Seismicity-based reservoir characterization for the stimulation of NM08. The top panel shows event-feedzone distances and the bottom shows the distance from each event to the center of the cluster of seismicity. Triggering fronts for diffusivities of $\mathrm{D}=0.03,0.09$ and $0.1 \mathrm{~m}^{2} / \mathrm{s}$ are shown for the start of phase one (blue curve) and the start of phase two (green). The orange curve starts at the switch from flow- to pressure-modulated injection for the top panel. In the bottom panel the orange curve starts at the onset of seismicity.

where $G$ is the modulus of rigidity, $\Delta V$ the cumulative injected volume and $M_{0}(\max )$ the maximum seismic moment (gray dotted line; Figure 3.15). For the stimulation and testing operations at NM08 and NM10 we have flow data for the entirety of the injections and can therefore place these points on the plot shown in McGarr (2014) and Goebel and Brodsky (2018, Figure 3.15). The Ngatamariki values for $M_{0}(\max )$ are comparable to values obtained from injection scenarios elsewhere. A key assumption 

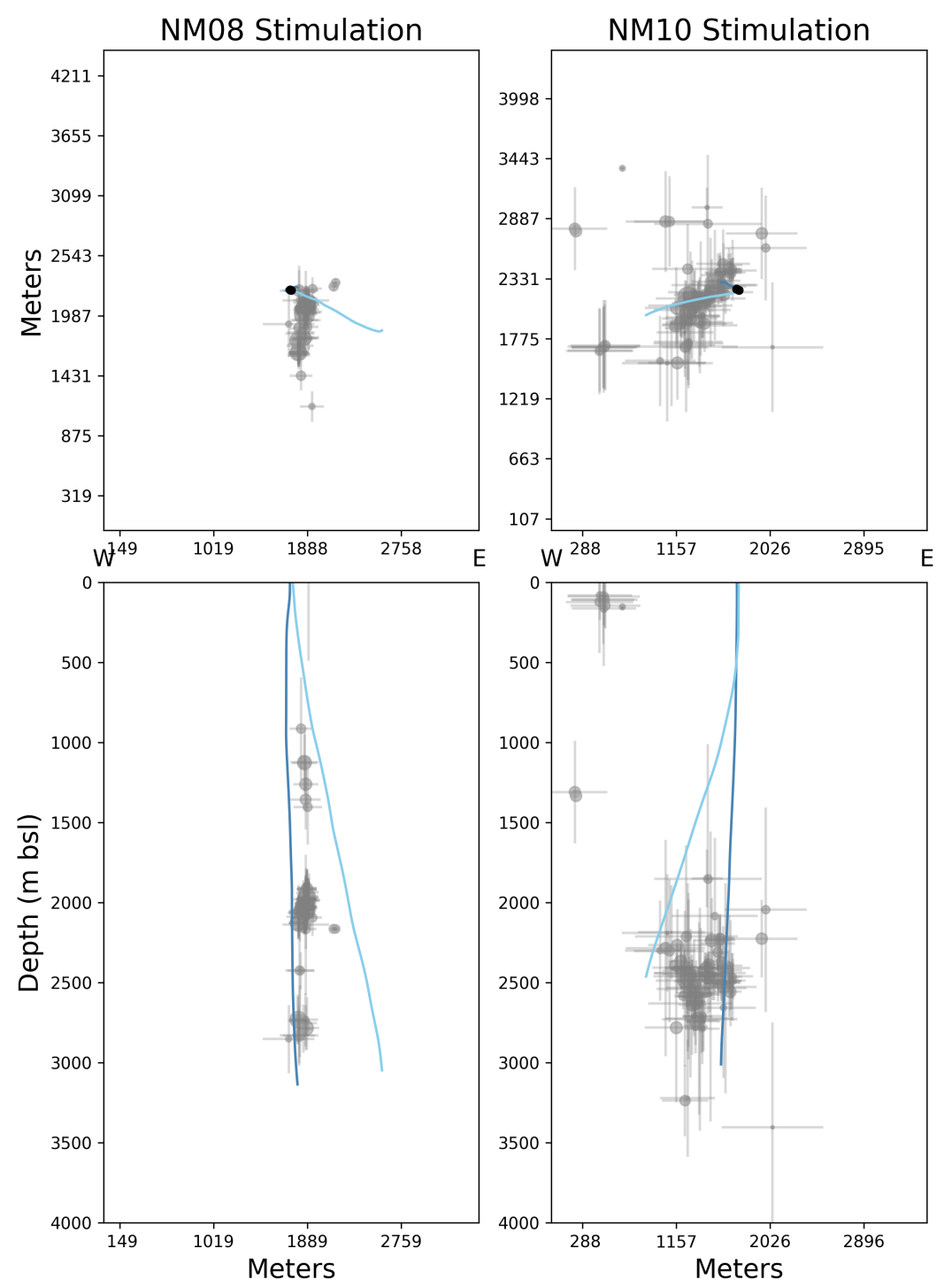

Figure 3.14: Hypocentral uncertainties for all events during the stimulations of NM08 and NM10 that were relocated with GrowClust. Error estimates are output as the median absolute deviation (MAD) of the distribution of the 50 bootstrap hypocenters for each event. The length of the crosses in the figure correspond to the horizontal and depth error values. Uncertainties in southern Ngatamariki are larger than in the north due to shifting station configurations and lack of stations during the drilling and stimulation of NM10.

inherent in the relationship of McGarr (2014) is that the earthquakes are induced by pore-pressure increase and that all induced deformation is seismic. However, we expect that the reservoir at Ngatamariki is contracting due to cooling, often aseismically. We therefore expect the $M_{0}(\max )$ of McGarr (2014) to be an overestimate. 


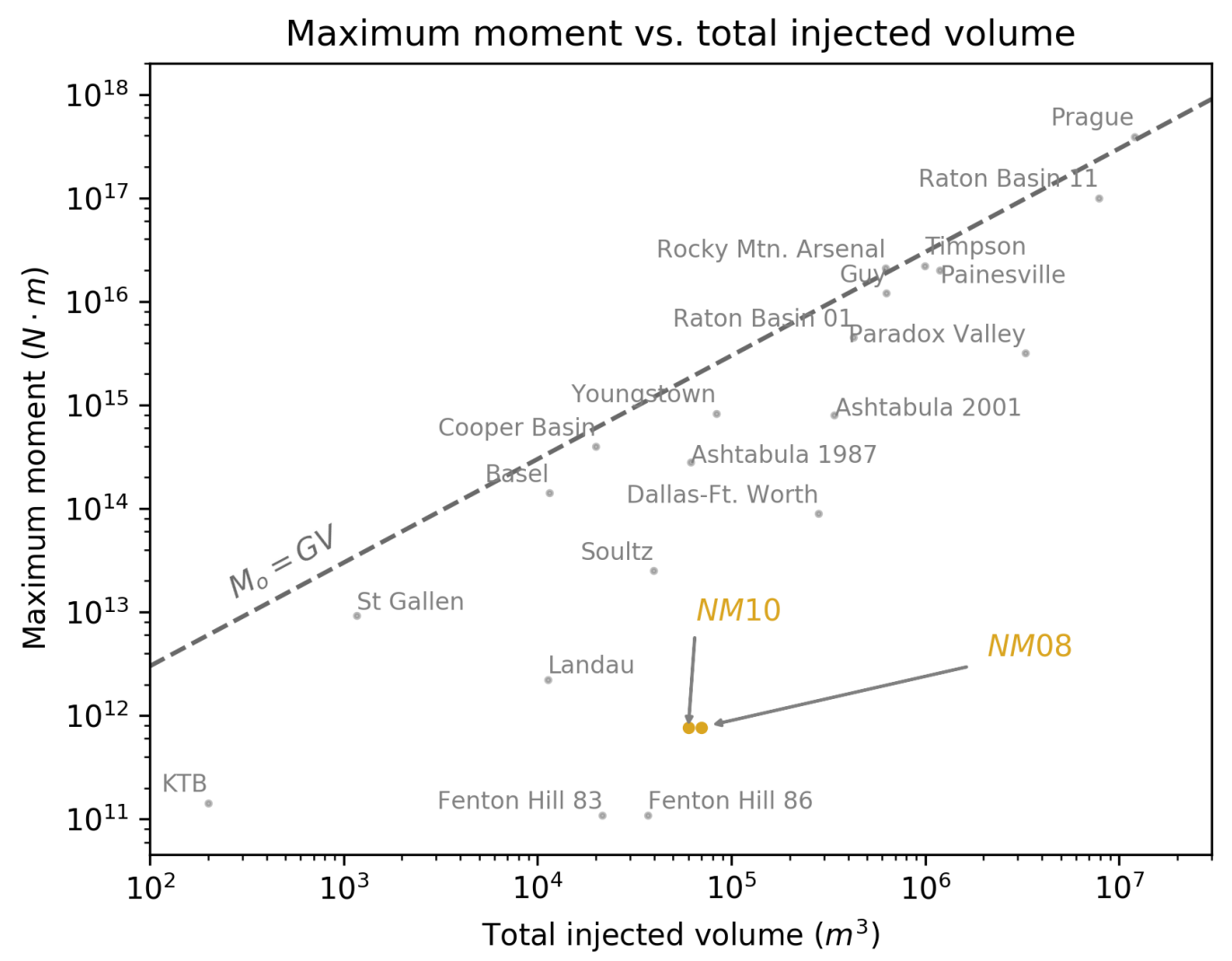

Figure 3.15: Maximum earthquake magnitude versus cumulative injected volume for the stimulation treatments at NM08 and NM10 as related to other similar injections worldwide. The dotted line shows the relationship determined by McGarr (2014). 


\title{
4 Seismic response to evolving injection at the Rotokawa geothermal field, New Zealand
}

\begin{abstract}
While simple spatial locations of seismicity can provide valuable insights about reservoir structure and fluid movement, frequency-magnitude distributions, often of secondary interest to operators, may contain unique information about the distribution of stress. In this work, we present a four-year catalog of seismicity for the Rotokawa geothermal field in the central Taupō Volcanic Zone, New Zealand starting two years after the commissioning of the 140 MWe Nga Awa Purua power station. Using waveformcorrelation-based signal detection we create a catalog of $>20000$ events that we use to update the location and orientation of two important reservoir faults. The rate of seismicity is insensitive to major changes in injection strategy during the study period, including the injectivity decline and shift of injection away from the dominant injector, RK24. We also map the spatial distribution of the earthquake frequency-magnitude distribution, or $b$-value. $b$-value in the area of the reservoir near major injection wells is $\sim 1.18$, but is less than 1.0 elsewhere, suggesting a positive correlation between $b$ and increased pore-pressure. $b$ also reveals a dependence on depth, increasing from $\sim 1.0$ at the depth of injection to nearly 1.5, two kilometers deeper. Such changes can potentially be used to monitor the relative distribution of pressure in the reservoir and may also hold clues to the distribution of differential stress, reservoir herterogeneity and degree of fracturing.
\end{abstract}

\subsection{Introduction}

Geothermal operators routinely monitor rates and locations of microseismic activity at developed reservoirs. Typically, the location of seismicity is assumed to correlate with regions where pore-fluid pressure has been artificially elevated by fluid injection (e.g. Sherburn et al., 2015; Garcia et al., 2016). In turn, these areas are assumed to correspond to major flow pathways in the reservoir, which are of paramount importance in understanding reservoir dynamics and planning injection/extraction well 
targets. In addition, reservoir structures can be accurately imaged from high-precision earthquake hypocentral locations, as can the extent of the permeable reservoir (Sewell et al. 2015b).

However, much of the information contained in even a basic earthquake catalog can go unused by reservoir managers. Specifically, while earthquake magnitudes are nearly always calculated during automated processing of seismic data, this information goes relatively unnoticed. In a limited number of cases, magnitude information has been used to infer reservoir properties such as pore-fluid pressure and the extent of fracturing. For example, Bachmann et al. (2012) modeled $b$-value and pore-fluid pressure for the case of the Basel enhanced geothermal injection well, showing that $b$ is expected to decrease exponentially with distance from a given injection point, but that $b$ actually increased between the wellbore and $200 \mathrm{~m}$ for reasons that weren't immediately clear. Given the inclusion of event magnitude data in nearly every earthquake catalog, more attention should be paid to patterns in frequency-magnitude distributions as a tool for reservoir management.

In this paper we focus on the Rotokawa geothermal field in the central Taupo Volcanic Zone (TVZ) of New Zealand. The field was initially developed in 1997 but has undergone large-scale development since that time, most significantly the commissioning of the 140 Megawatts electrical (MWe) Nga Awa Purua (NAP) plant in 2010 (McNamara et al., 2016). The seismic dataset analyzed here (2012-2015) corresponds to a period of stabilization, during which the reservoir was equilibrating to the increased extraction required by the installation of NAP. We use a matched-filter earthquake detection technique to substantially increase the number of events in our catalog relative to standard, automatic detection. We then calculate magnitudes for the newly-detected events, again using a waveform correlation-based technique before precisely relocating each event.

Over its two decades of development, the Rotokawa reservoir has been relatively well studied. The current understanding of the reservoir is based on research from a number of workers who have identified at least four compartments, which are likely bounded by a number of faults acting as barriers to inter-compartment fluid flow (Sewell et al. 2015a; Addison et al., 2017; Wallis et al., 2013). Previous studies of microseismicity at Rotokawa have been used to constrain the location of one of these structures, the Central Field Fault, but due to its confinement to the injection field compartment, seismicity has been unable to reveal further structure within the reservoir Sherburn et al., 2015; Sewell et al., 2015b).

In the following analyses, we compare our catalog to the catalogs of previous studies of microseismicity at Rotokawa (Sewell et al., 2015b; Sherburn et al., 2015) and relate the rate and location of seismicity to changes in injection strategy at Rotokawa. We also map the frequency-magnitude distribution ( $b$-value) within the field, something which has not been done at Rotokawa before. While the observed patterns in $b$-value 
are quite complex, when combined with high-precision locations, they show potential for mapping of pore-pressure and/or fracturing extent at a reservoir scale.

\subsubsection{Rotokawa resource development}

As with most of the TVZ geothermal fields, the existence of the Rotokawa resource was originally verified through a New Zealand government-funded drilling program starting in the 1960's (Cole and Legmann, 1998). The first resource consent was granted to the Tauhara No. 2 Trust and various other entities in 1993 with electricity generation beginning in 1997 with the installation of the 24 MWe Rotokawa A (RGEN) combined-cycle power plant Legmann and Sullivan, 2003). In 2000, Mercury NZ Ltd. (then Mighty River Power) combined with the Tauhara No. 2 Trust to form the Rotokawa Joint Venture, which continues to oversee development at Rotokawa at present Legmann and Sullivan, 2003).

Prior to 2005, reinjection at Rotokawa was done at depths of $<1000 \mathrm{~m}$ Sewell et al., 2015a) but was moved to greater depths (1000-3000 m) due to pressure buildup in the shallow injection zone (McNamara et al., 2016). Additional resource consents were granted to the Rotokawa Joint Venture in 2007, prompting the drilling of more than ten additional wells (RK19-RK35) and culminating in the commissioning of the NAP plant in 2010, bringing the total installed capacity at Rotokawa to 174 MWe fed by 60,000-65,000 t/d of fluid from the reservoir (McNamara et al., 2016). The additional information provided by the new wells allowed for significant improvements to the reservoir conceptual model detailed by Sewell et al. (2015a) and McNamara et al. (2016).

\subsubsection{Rotokawa operations changes}

The dataset analyzed in this work spans the four years starting in 2012 and ending at the end of 2015. During this period, little new development was undertaken at Rotokawa, as the resource was adjusting to the significant increase in production associated with the startup of NAP in 2010. Changes in injection and production are therefore more subtle than those analyzed at the nearby Ngatamariki field for the same period, where drastic changes were associated with well stimulation and power plant commissioning (Clearwater et al. 2015). However, Mercury have identified a number of periods from 2012-2015 during which the character of seismicity may help address various outstanding questions about the nature of the reservoir:

As is typically the case for produced reservoirs of any type (e.g. natural gas, oil, geothermal), seismicity at Rotokawa is caused predominantly by the injection, not the extraction, of fluids. This is because injection increases pore fluid pressure, thereby destabilizing fracture networks, whereas extraction achieves the opposite, although 

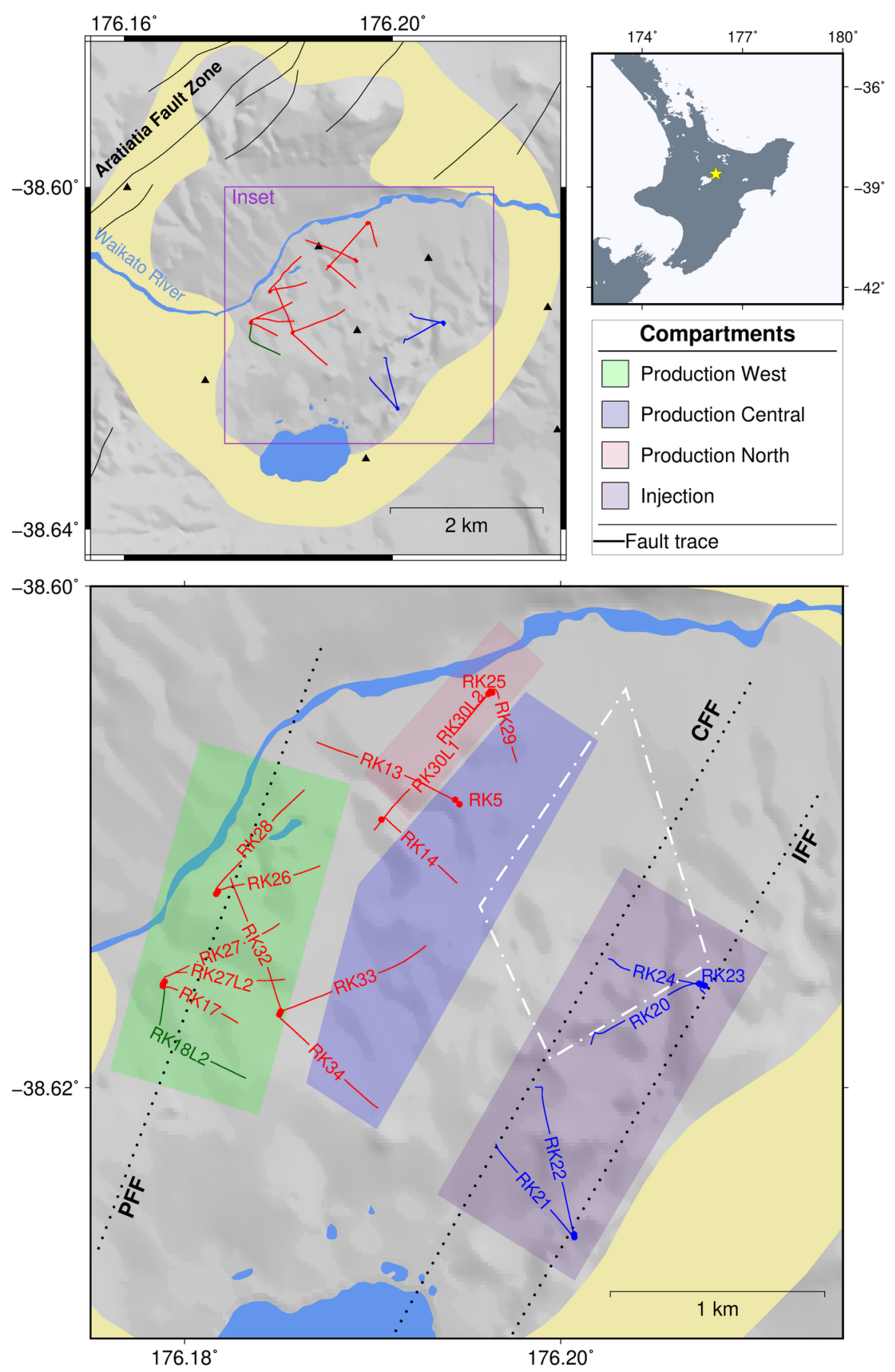

Figure 4.1: Overview of the Rotokawa geothermal field. The top right panel shows the location of the field on the North Island of New Zealand (yellow star). The top left panel shows the resistivity boundary of the field in yellow and injection and production wells in blue and red, respectively. Solid black lines are active faults. The lower panel shows a closeup of the field, with the well names labeled and the three known faults $(\mathrm{PFF}$, CFF, IFF shown as black dotted lines. The white dot-dashed line shows the extent of significant seismicity from 2008-2012, as reported by Sherburn et al. (2015). There are four known compartments in the Rotokawa reservoir shown as colored polygons. Each is semi-isolated from the others by either a permeability contrast or impermeable barrier (i.e. a fault). The production field comprises three compartments, the west, central and north (green, blue and red, respectively). The injection field is shaded in purple and is a separate compartment. 


\begin{tabular}{cccc} 
Field & Operation & Start & End \\
\hline Rotokawa & RK24 Injectivity Decline & 2012 & 2013 \\
Rotokawa & Switch of Injection: RK24-RK23 & $2014-6$ & $2014-7$ \\
Rotokawa & RK34 Drilling Losses & $2014-9$ & $2014-11$ \\
Rotokawa & Plant Shutdowns and Startups & $2012-2015$ & $2012-2015$
\end{tabular}

Table 4.1: Period of interest defined by Mercury for 2012-2015

stress changes induced by the removal of reservoir volume are capable of triggering earthquakes (Segall, 1989; National Academy of Sciences, 2013). For this reason, the periods defined by Mercury correspond predominantly to changes in injection operations. The first two periods in Table 4.1 correspond to a period of injectivity decline in the dominant injection well RK24 (Figure 4.1) due to an unknown cause. In response to the well's declining ability to accept injectate from the power plants, excess fluid was shifted to well RK23 (Figure 4.1).

From September to November 2014, Mercury drilled an additional production well, RK34. At reservoir depths, the drilling operation incurred full fluid losses. Similar drilling losses induced a large number of seismic events during drilling in southern Ngatamariki (Hopp et al., 2019, Chapter 3) and may have had a similar effect at Rotokawa. Finally, as noted by Sewell et al. (2015b), swarm-like behavior (which they defined as any day on which more than 15 events occurred) has been observed at Rotokawa in the past and is thought to be related to pressure perturbations induced by power plant shutdown and startup during regular maintenance operations.

The fluid injected at Rotokawa varies by application and between individual wells. For instance, during the drilling of RK34, the drilling fluid was composed predominantly of water at an ambient temperature of $10-20^{\circ} \mathrm{C}$. During standard plant operations, the injectate at each injection well can be condensate (the product of cooling the steam used to turn the turbines), concentrated brine (brine with condensate removed) or some combination of these end members. During most of the study period, brine and condensate from NAP were injected into RK23-24, while the injectate from RGEN was injected into RK20. Depending on the mixture, fluid entered the injection wells at a temperature between $\approx 40^{\circ}$ and $130^{\circ} \mathrm{C}$.

\subsubsection{Reservoir model}

The behavior of the Rotokawa reservoir is dominated by three known NE-SW striking faults, each named for its location relative to the injection or production field. From east to west they are: the Injection Field Fault (IFF), Central Field Fault (CFF) and Production Field Fault (PFF) (Wallis et al., 2013, Figure 4.1). The existence and orientation of these faults is known from well cuttings that show vertical offsets in the top of the Rotokawa Andesite (and other units) between wells. The CFF, for instance, 
is credited with a vertical displacement of nearly 400-500 m between the injection and production wells, while the $\overline{\mathrm{IFF}}$ and $\mathrm{PFF}$ represent vertical displacements of 250 $350 \mathrm{~m}$ (Wallis et al. 2013). A number of independent datasets have been used to corroborate the presence of these faults, including tracer returns Addison et al. 2015. 2017), pressure compartmentalization (Quinao et al., 2013; Sewell et al., 2015a) and previous studies of microseismicity (Sherburn et al., 2015; Sewell et al., 2015b).

Large lateral pressure gradients within the Rotokawa reservoir indicate that it is made up of several discrete compartments that either have different permeabilities or are separated by structures that act as cross-strike flow barriers Quinao et al. 2013 . Sewell et al., 2015a). The major faults mentioned above act either as barriers between these compartments or as conduits for fluid flow within compartments. Slow or nonexistent tracer returns from injection to production indicate that the $\mathrm{CFF}$ is such a barrier, isolating the injection compartment from each of the production compartments (Figure 4.1). However, this isolation is not constant along the strike of the CFF Addison et al., 2017). Current modeling by Mercury suggests that a 'leaky' connection exists between the main injection well, RK24 and the central production wells (RK5, RK14, RK29, blue polygon in Figure 4.1), which provides pressure support to these production wells. This pressure support is not present in the western (4.2 MPa drawdown) or northern (3 MPa drawdown) production field compartments Quinao et al. 2013; Addison et al., 2017, green and red, respectively in Figure 4.1).

Strong pressure connection between the wells within the western production compartment suggests that the $\overline{\mathrm{PFF}}$ acts as an along-strike fluid conduit, transmitting pressure signals quickly to distances of up to a kilometer Quinao et al., 2013; Sewell et al. 2015a; McNamara et al., 2016). These pressure observations are well correlated with the geologic offsets observed in the well cuttings mentioned above Wallis et al. 2013).

There is less evidence to suggest what role the IFF may play in reservoir behavior, as the only well completed to the east of this structure is RK23. Tracer was injected into RK23 in 2011, but issues with isotope breakdown rendered much of the test inconclusive. Bottom hole temperatures in RK20/24 are some $40^{\circ} \mathrm{C}$ higher than in RK23, only $\sim 200 \mathrm{~m}$ away, suggesting some sort of lateral variation in reservoir properties between the wells (e.g. permeability). However, some evidence suggests that there is also a significant pressure connection between all three injection wells Quinao et al. 2013). RK23 has traditionally accepted a smaller proportion of overall injection, so trends associated with changes there may be difficult to separate from the rest of the injection wells. 


\subsubsection{Previous work}

The only previous studies of the seismicity at Rotokawa were conducted for the years 2008-2012 by workers at GNS Science and Mercury Sherburn et al., 2015; Sewell et al. 2015b). These studies detailed a pattern of seismicity that shifted with time as the injection and production strategies evolved in response to field development and reservoir understanding. At of the end of 2012, Sherburn et al. (2015) identified the currently-active area of seismicity as that bounded by the white, dot-dashed diamond shown in Figure 4.1. This cluster of seismicity occurred at the approximate depth of the permeable zones in wells RK20, RK23 and RK24, which accounted for nearly all of the deep injection into the Rotokawa reservoir at the time. This cluster was inferred to be bounded to the northwest by the CFF (Figure 4.1), corroborating the evidence from tracer testing that fluid flow was impeded across the structure, thus allowing pressure buildup and reservoir cooling east of the fault, thereby inducing seismicity Sherburn et al., 2015, Sewell et al., 2015b).

Sherburn et al. (2015) and Sewell et al. (2015b) cited the relatively modest wellhead pressures measured in the injection field $(<1.5 \mathrm{MPa})$ as evidence for cooling-dominated, and not pressure-dominated, triggering of seismicity at Rotokawa. However, we feel that evidence is limited to support this claim, given that stress changes as low as $10^{-2}$ MPa have been shown to trigger seismicity in many settings Stein, 1999; Keranen and Weingarten, 2018). As Sewell et al. (2015b) noted, the large temperature gradients induced at the injection wells $\left(>180^{\circ} \mathrm{C}\right)$ certainly produce stress changes of tens of MPa within 10-100s of meters of the well, especially when large volumes are injected over years Stephens and Voight, 1982). Though the effect of cooling on fracture stability is complex (Jeanne et al., 2015b), these cooling effects likely do dominate earthquake triggering close to the injection wells. However, the occurrence of seismicity beyond $\mathrm{a} \sim 500 \mathrm{~m}$ radius and the lack of measured cooling at the production wells (including those that receive pressure support from the injection zone) indicates that other effects, including pore-pressure increase and poroelastic stress transfer also play a role in earthquake triggering at Rotokawa (Schoenball et al., 2012).

\subsection{Data and methods}

The earthquake catalogs for Ngatamariki and Rotokawa are subsets of the same catalog and were processed as one. For ease of analysis, they were then separated into individual catalogs for each field. Therefore, the methodology described in Chapters 2 and 3 is identical to the methods applied here. For a detailed description of these methods, the reader is referred to those Chapters, while here we provide only a brief summary. 


\subsubsection{Data}

The Mercury seismic network covers an area of approximately $450 \mathrm{~km}^{2}$, consisting predominantly of short-period geophones but incorporating a number of GeoNet broadband instruments as well. From the initial earthquake catalog provided by GNS Science, we discarded events located further than $5 \mathrm{~km}$ from the Rotokawa resistivity boundary (yellow shaded area, Figure 4.1) as well as events with average pick residuals within one standard deviation of the mean pick residual for the entire catalog. The remaining 3225 events $\left(\mathrm{M}_{L}\right.$ 0.37-3.68) were relocated using NonLinloc Lomax et al. 2014) and used as template events in the matched-filter detection routine.

\subsubsection{Matched-filter detection}

Each template consists of one-second-long waveforms, starting 0.1 seconds before the P-pick only on vertical channels. Both raw and template waveforms were sampled at 50 $\mathrm{Hz}$ and filtered from 3.0 to $20.0 \mathrm{~Hz}$. Templates were cross-correlated with continuous data at 50 samples per second and detections were recorded whenever the detection statistic exceeded exceeded its daily median absolute deviation $\times 8$ (as suggested by Shelly et al., 2007; Matson, 2019). Finally, duplicate detections within 2 s of another detection were removed.

\subsubsection{NonLinLoc locations}

P-picks were made for each new detection via cross-correlation with the template over a 0.2 second window centered at the detection time. Picks for which the cross-correlation coefficient was less than 0.4 were discarded, as were events with five or fewer picks. S-picks were made for the remaining events using the method of Diehl et al. (2009) and modified by Castellazzi et al. (2015). Events were located with NonLinLoc Lomax et al., 2014) using the 1-D velocity model shown in Table 2.3.

\subsubsection{Magnitudes}

The magnitude for each newly-detected event was calculated based on its amplitude relative to the template that detected it. Because the waveforms are inherently similar between template and detection, we assume that their relative amplitude also represents their relative moment (Shelly et al., 2016a). We perform the singular-value decomposition of these waveforms, from which the relative amplitude is calculated as the ratio of the first and second elements of the first output basis vector (see Figure 2.10 for details). The relative moment was calculated as the median of a minimum of four relative amplitudes. 
We then calculated the seismic moment for each template event using the GNSScience-calculated $\mathrm{M}_{L}$ using the $\mathrm{M}_{L}-\mathrm{M}_{w}$ scaling relationship of Ristau et al. $(2016)$ and the well-known $\mathrm{M}_{w}$-to-moment relationship of Hanks and Kanamori (1979). This process was used to calibrate the relative moment values to actual values of $\mathrm{M}_{L}$. After applying this methodology, 28,414 events remained in our catalog.

\subsubsection{GrowClust locations}

Finally, the entire catalog was relocated using the double-difference relocation program GrowClust (Trugman and Shearer, 2017) with differential travel times generated using the Python package hypoDDpy (Krischer, 2015) using a 1 s correlation window, a maximum time shift of $0.2 \mathrm{~s}$ and a minimum cross-correlation value of 0.6. The final 6479 double-difference relocations are shown in Figure 4.3.

\subsection{6 $b$-value calculation}

The frequency-magnitude relationship for earthquake catalogs is a power-law distribution described by Gutenberg and Richter (1942) as

$$
\log N=a-b M
$$

where $a$ increases with the number of earthquakes in the catalog and $b$ describes the distribution of earthquake magnitudes above the catalog magnitude of completeness $\left(M_{c}\right)$. We calculate $M_{c}$ for the catalogs presented here following the methodology of Wiemer (2000). For a range of $M_{c}$ values, $a$ and $b$ are determined for our earthquake catalog and an idealized synthetic distribution constructed with the same $a$ and $b$ values. The absolute difference between the synthetic and measured distributions given by Wiemer (2000) is:

$$
R\left(a, b, M_{i}\right)=100-\left(\frac{\sum_{M_{i}}^{M_{M A X}}\left|B_{i}-S_{i}\right|}{\sum_{i} B_{i}} 100\right)
$$

where $B_{i}$ and $S_{i}$ are the observed and predicted number of events in a given magnitude bin, $i$. This residual is then minimized to find the most appropriate $M_{c}$ (Figure 4.2).

We then map $b$-value in space using the approach of Bachmann et al. (2012), wherein, for each event in the catalog (which they refer to as a 'focus'), the nearest $n$ events are selected and $M_{c}$ is calculated for this subset. During initial testing of this procedure, $b$ and $M_{c}$ were colinear, implying that we were introducing a bias where an increasing $M_{c}$ led to a higher $b$-value. To eliminate this bias, we compare the $M_{c}$ of each sub-catalog with that of the catalog as a whole (0.74). If the sub-catalog $M_{c}$ is greater than that of the entire catalog, we discard it. If the sub-catalog $M_{c}$ is less than that of the entire catalog, we then enforce a $M_{c}$ of 0.74 . If the number of events above 


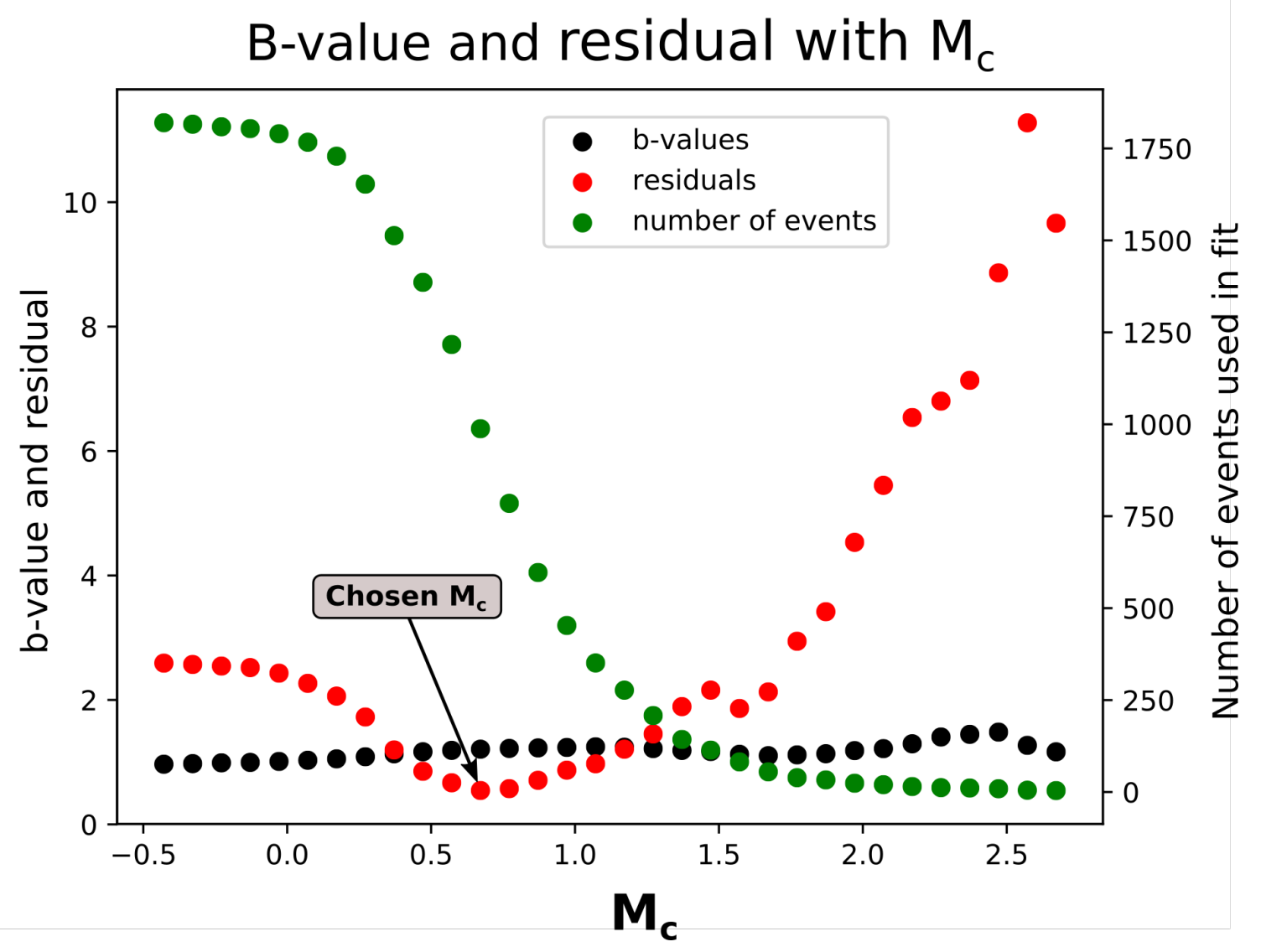

Figure 4.2: Illustration of the magnitude of completeness calculation suggested by Wiemer (2000). Red dots indicate the normalized, absolute difference between the observed and theoretical distribution of events for a given $\mathrm{M}_{c}$ and $b$-value. Black dots are the calculated $b$-value and green shows the total number of events used for the fit at each magnitude of completeness.

this $M_{c}$ exceeds a threshold, the $b$-value is calculated and mapped to the focus event using the maximum-likelihood method (Aki, 1965; Shi and Bolt, 1982). Bachmann et al. (2012) map the $b$-value for the 150 nearest events, with a threshold of 25 events greater than $M_{c}$. Here we use the nearest 300 events with a minimum threshold of 100 events above $M_{c}$.

One possible, yet poorly understood, complication in calculating $b$-values for our catalog arises due to the use of the matched-filter detection routine. A template event can only detect events with the same (or highly similar) location and fault plane solution. Therefore, if an event occurs in a location and/or with a focal mechanism solution for which there is no analog in the template catalog, it will go undetected. This may give rise to a bias in the final matched-filter catalog that would increase the uncertainty in calculating $\mathrm{M}_{c}$ and $b$-value. For the case of Ngatamariki and Rotokawa, although our template catalog was filtered to exclude events with high pick residuals (and therefore unreliable locations), we do not appear to have introduced any spatial bias, as shown by the similar distribution of template events relative to the raw, un- 
filtered catalog (see Chapter 2; Figure 2.3). Also, because the template events were drawn from the exact time span over which we conducted the correlation detection, we do not anticipate having introduced a temporal bias.

The final possible bias is that the template catalog is devoid of events having a particular focal mechanism. Assuming a lack of other biases, as stated above, the only way for this to have happened is if a particular mechanism occurred only at small magnitudes below the original amplitude-based detection threshold $\left(\mathrm{M}_{c} \sim 0.7\right)$. However, due to the elevated pore-pressures present at actively-produced geothermal fields, we expect a scenario in which a variety of fracture orientations are activated, not only those corresponding to critically-stressed fractures, meaning that the potential focal solution space within the reservoir stress field should be relatively well-sampled in the template catalog. For a particular focal mechanism solution to be missing from the template catalog, it would likely need to have been $<\mathrm{M}_{L} 0.7$ and on a fracture oriented differently from the dominant NE-SW striking pattern in the reservoirs. It may be that such events correspond to new, tensile fractures induced by cooling-related contraction, which may produce such orientations and small sizes (Stephens and Voight, 1982; Wiemer et al., 1998) but we have difficulty envisioning any alternative scenario.

\subsection{Results}

\subsubsection{Locations}

The GrowClust relocations of the final 6479 events are shown in Figure 4.3. From a field-wide perspective, the location of seismicity has changed little from that presented by Sherburn et al. (2015) and Sewell et al. (2015b). The area of seismicity identified in their studies is overlain on Figure 4.3 (black dot-dashed diamond) for context and broadly agrees with the extent of the densest seismicity in our catalog. Most events occur in the northeastern portion of the field, between the northern injection wells (RK20, RK23, RK24; Figure 4.1) and the northern production wells (RK13, RK14, RK25, RK29, RK30; Figure 4.1), with some events extending further towards the north and east. The northwest portion of the field, north of the Waikato River, as well as the southern injection zone (near wells RK21-22) correspond to areas of little seismicity. Within the area of densest seismicity, however, our catalog more clearly reveals subclusters and structure than previous studies, which identified only one uniform area of dense seismicity (Sherburn et al., 2015).

\section{Location uncertainties}

There are obvious differences between the hypocenters presented here (2012-2015) and those presented previously (Sherburn et al., 2015, Sewell et al., 2015b, 2008-2012). 

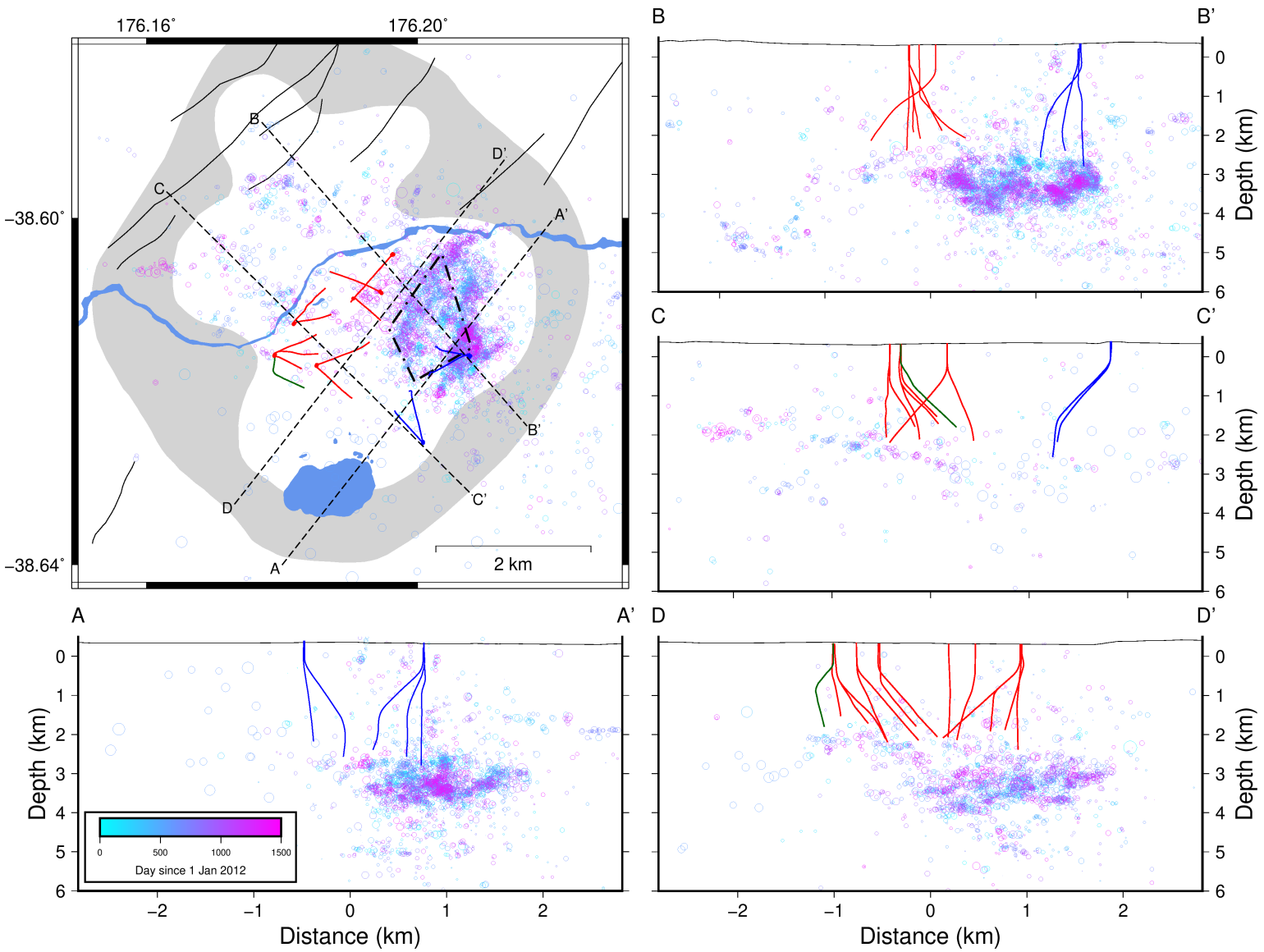

Figure 4.3: Seismicity at Rotokawa for the years 2012-2015 colored by date of occurrence. Blue events occurred earlier in the dataset and pink events occurred later. Four cross sections are plotted to show the depth distribution of seismicity and their surface projections are shown in map view. The dot-dashed diamond indicates the area of dense seismicity identified by Sherburn et al., 2015; Sewell et al., 2015b for the 2008-2012 catalog.

While it is possible, that these location differences represent a real migration of seismicity, there are at least two other potential causes for this, which we will discuss in detail. The first is a difference in the location algorithm used to obtain the finalized, double-difference locations, and the other is the significant uncertainty in the velocity structure at the geothermal fields.

In order to illustrate the potential contribution of the different location algorithms to the variations in earthquake hypocenters, we relocated a subset of an earlier GNS Science catalog containing only manual phase picks using two algorithms: GrowClust (used in this study Trugman and Shearer, 2017) and TomoDD (Sherburn et al., 2015, Sewell et al., 2015b; Zhang and Thurber, 2003). The locations for which are shown in Figure 4.4. The velocity model used to obtain the GNS Science locations was an unpublished 3D model for Rotokawa (Sherburn et al. 2015). For the GrowClust locations, we located the catalog from scratch with NonLinLoc (Lomax et al., 2014), 
using the velocity model from Sewell et al. (2017, Table 2.3), before relocating the catalog with GrowClust.

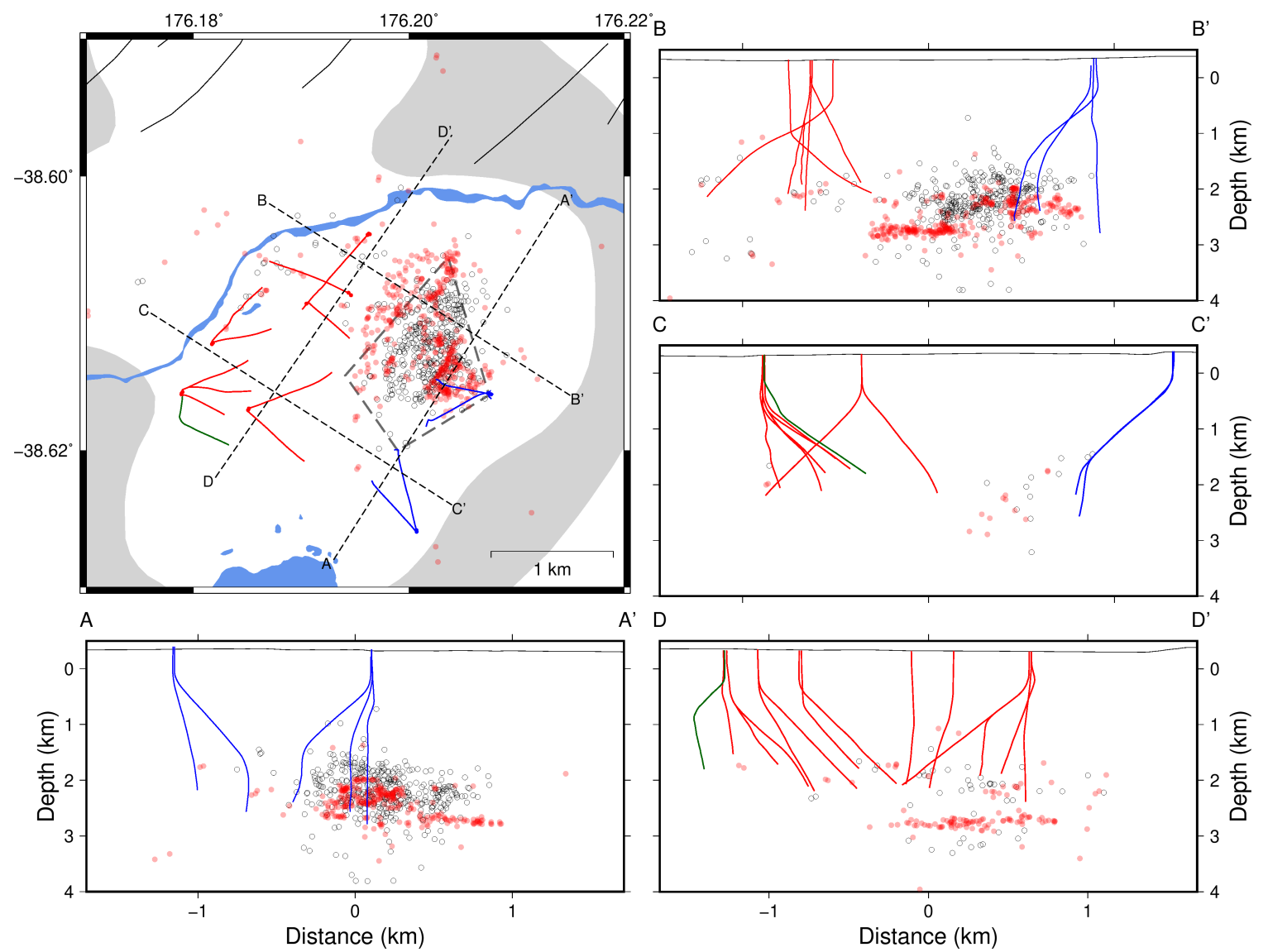

Figure 4.4: GNS Science manual phase picks for 2012-2013, relocated with GrowClust (red circles), and by GNS Science with TomoDD (black, unfilled circles). The dark gray, dashed diamond identifies the area of active seismicity as of the end of 2012, defined by the TomoDD locations of Sherburn et al. (2015). The GrowClust hypocenters are more tightly clustered, but occupy a larger overall footprint than the GNS Science TomoDD locations.

As stated above, the locations provided by GNS Science broadly agree with the depths of the major feedzones for the main injection wells $(<2000 \mathrm{~m} \mathrm{bsl})$, particularly RK24, although a number of events occur at shallower depths (Figure 4.4). Our GrowClust locations cluster more tightly, especially in cross-section. Those events occurring nearest RK24 are in better agreement with the depth of the feedzones than the TomoDD locations. In map view, these events also define a structure striking NESW, consistent with the regional trend and perhaps associated with the Injection Field Fault, previously inferred from stratigraphic offsets between the three main injection wells RK20, RK23 and RK24 (Sewell et al., 2015a).

While the GrowClust locations are more consistent with known structures and feedzone depths in the deep reservoir, it is nevertheless difficult to state with certainty that they are the 'correct' locations. It is particularly important to note that double- 
difference locations methods are known to produce location 'streaking', or elongation of actual features, due to unmodeled variations in the velocity model (Michelini and Lomax, 2004). For the case presented here, we use a 'layer-cake' 1-dimensional velocity model, which is particularly susceptible to streaking of hypocenters, as shown by Michelini and Lomax (2004). A possible artifact of this effect can be seen in the red GrowClust location in Figure 4.4, cross-sections A-A', B-B' and C-C'. Therefore, Figure 4.4 simply serves to show that, using the same data, variations in velocity model and location algorithm can produce hypocenters that may yield different interpretations. We are conscious of these uncertainties but continue with the analyses of the GrowClust locations.

\subsubsection{Magnitudes}

In Figure 4.5, we show the frequency-magnitude distribution for the GNS Science template events (dashed) and final detected events (solid) for which we calculated a $b$-value of $\sim 1.27 \pm 0.01$, comparable to $b$-values in areas of hydrothermal activity worldwide, and similar to those calculated at the Ngatamariki geothermal field, located $\sim 7 \mathrm{~km}$ to the north (Figure 4.5b) (e.g. Bachmann et al., 2012; Wiemer and McNutt, 1997; Dinske and Shapiro, 2012; Hopp et al., 2019, Chapter 3). In areas of active volcanism, as well as in both hydrocarbon extraction and geothermal settings, $b$-values far exceeding 1.0 are reported (Dinske and Shapiro, 2012; Shapiro et al., 2011). In general, b-values above 1.0 have been attributed to the presence of fluids, high pore-fluid pressures and material heterogeneity. Elevating the pore pressure allows fractures to fail that are not optimally-oriented in the local stress field. These fractures experience smaller differential stress than those which are critically stressed, and will therefore produce smaller-magnitude earthquakes when they fail (Bachmann et al., 2012). It has also been suggested that the presence of a strong thermal gradient (e.g. related to an intrusion or injection of cold water) could aid in development of small, tensional fractures Warren and Latham, 1970), thereby increasing the population of small fractures, which can only fail in small-magnitude events. Both the elevation of pore pressure and the application of a strong thermal gradient may increase the frequency of small-magnitude seismic slip and therefore raise the $b$-value above naturally-occurring values (i.e. $\sim 1.0$ ).

\subsection{3 $b$-value mapping}

Figure 4.6 shows $b$-value in map view and cross-section. There are no immediatelyevident spatial trends in $b$, which instead shows a complex, heterogeneous distribution varying from $\sim 0.5$ to $>1.5$. $b$-values of $>1.5$ are found throughout the reservoir, including north of the Waikato river. 

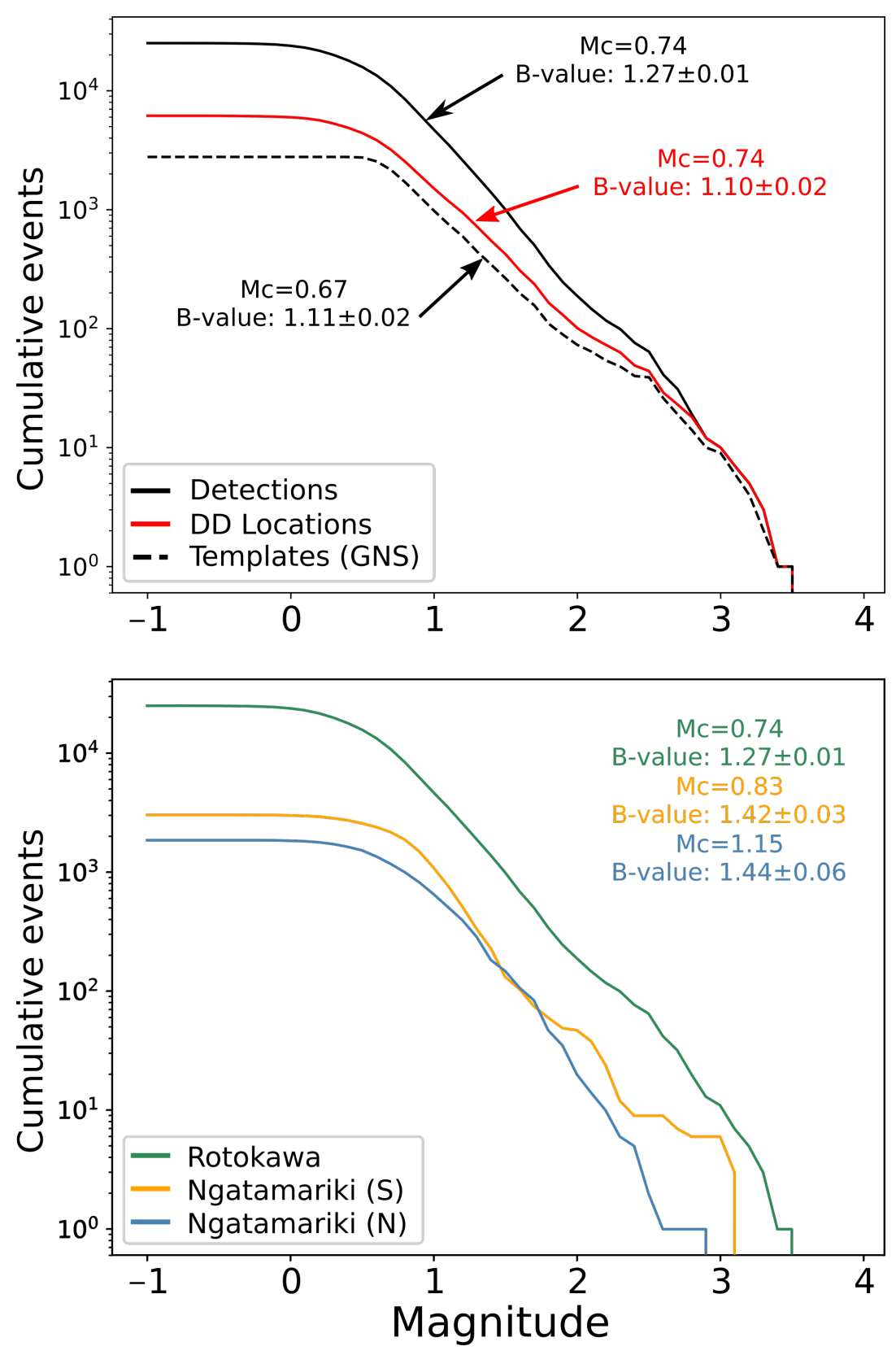

Figure 4.5: Top panel: Frequency-magnitude distributions for the GNS Science (dotted), matched-filter-detected (solid, black) and GrowClust-relocated (solid, red) catalogs at Rotokawa from 2012-2015. The calculated magnitude of completeness and $b$-value are indicated and color coded to each catalog. Bottom panel: Frequencymagnitude distributions for the full matched-filter catalog at Rotokawa compared to northern and southern Ngatamariki. The $\mathrm{M}_{c}$ and $b$-value for each catalog are noted in the top right, color coded to the corresponding curves. 


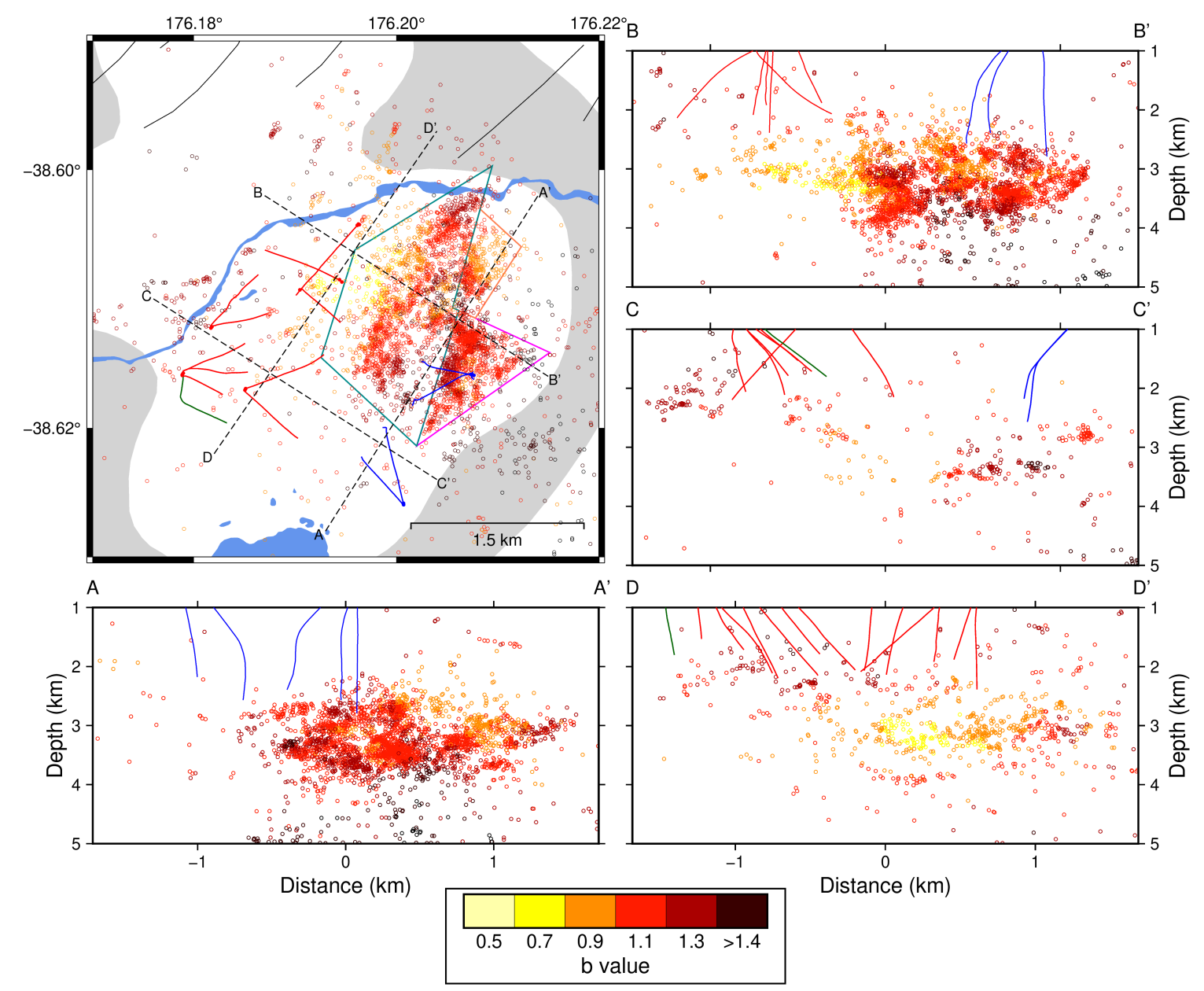

Figure 4.6: Spatial variation in $b$-value at Rotokawa. Events are colored by $b$-value, calculated using the nearest 300 events, provided there are at least 100 events greater than the $\mathrm{M}_{c}$ of 0.74 . The western (green polygon), northeastern (coral polygon) and southeastern (pink polygon) compartments are defined from hypocentral locations and will be relevant to the following discussion.

\subsection{Discussion}

\subsubsection{Compartmentalization}

As shown in Figure 4.1 and discussed in Section 4.1.3, the Rotokawa reservoir is known to be broken into a number of compartments, at least three in the production field and one in the injection field. Patterns in seismicity are of little use in delineating the borders of the production field compartments because relatively few earthquakes occur in that portion of the field. However, we think that our relocations (Figures 4.3 and 4.7 ) allow for further constraint on the location of the Central Field Fault and Injection Field Fault, and may provide evidence for previously unidentified subcompartments within the injection field. 
There are at least two NE-SW-striking sub-linear features revealed by this catalog, outlined and labeled in bold red in Figure 4.7. One feature lies between the injection and production fields, taking on an arcuate shape, concave towards the southeast. This structure likely defines the $\mathrm{CFF}$ as it sits between the injection field and the central production compartment, as previously modeled by Wallis et al. (2013). The other structure strikes NE-SW between wells RK20/24 and well RK23, and extends towards the northeast. We therefore interpret this to be the IFF, again due to the consistency of its location with known geologic and temperature offsets. The IFF, specifically, was not previously imaged by microseismicity, nor has the offset between what appear to be CFF-related and IFF-related events been previously identified (Sherburn et al., 2015). We suggest three possible reasons for this:

- One, we are able to more clearly define structures as a result of the larger number of events in our catalog;

- Two, the change in algorithm used for the double-difference relocation (GrowClust, in our case) was more stable in the presence of noisy data than TomoDD (see Section 4.3.1);

- Three, the location changes may actually reflect reservoir-scale changes in permeability and fluid flow from 2012 until the end of 2015.

We cannot rule out the possibility of large-scale migrations in the seismically-active portion of the reservoir. For example, deepening of seismicity has been observed at The Geysers geothermal field (e.g. Martínez-Garzón et al., 2014; Jeanne et al., 2015a). However, as shown in Figure 4.4, changing only the data processing and algorithm for a single set of phase picks is enough to produce significant changes in the final locations. In addition, the GrowClust locations using GNS Science manual phase picks (red dots, Figure 4.4 resemble our final locations using automatic and cross-correlation-derived picks (Figure 4.3), with two separate clusters of seismicity aligned NE-SW. The means that the revealed structures are independent of data quality, given that the manual picks are more precise than our automatic picks. Finally, the two separate structures make sense geologically, given the known locations of both the CFF and IFF from well cutting offsets. For these reasons, we prefer our locations to those published previously by GNS Science (Sherburn et al., 2015).

A third potential structure is outlined in dotted red striking NW-SE (Figure 4.7). On visual inspection, this feature is less obvious than the CFF and $\mathrm{IFF}$ and we therefore assign less confidence to its existence. Furthermore, no major cross-strike (NW-SE) structures have been identified from offsets in well cuttings. However, along-strike variations in pressure drawdown and tracer returns do indicate that the reservoir is likely divided not only by NE-SW-striking structures, but by NW-SE-striking structures as well (Sewell et al., 2015a; Quinao et al., 2013). Specifically, the contrast between the 
$>4 \mathrm{MPa}$ drawdown in the western production field (green box, Figure 4.1) and the 3 $\mathrm{MPa}$ drawdown at the northern production wells (red box, Figure 4.1) suggests the existence of NW-SE structures.

The following analysis is based on the hypothesis that pressure differentials between compartments may exhibit as variations in seismic characteristics. Therefore, we have divided the area of densest seismicity into three potential compartments bounded loosely by the inferred locations of the major faults and the potential NE-SW structure mentioned above (Figures 4.6 and 4.7). The light green polygon in Figure 4.7 will be referred to as the western compartment and the coral and pink polygons will be referred to as the northeastern and southeastern compartments, respectively. These divisions are based on hypocentral locations only and constitute potential compartments that have not been identified previously.

\subsubsection{Compartment characteristics}

For a first-order view of the properties of seismicity in the compartments defined in Figure 4.7, we plot the cumulative number of events as well as the normalized (by the total number) cumulative number of events in each (Figure $4.8 \mathrm{a}-\mathrm{b}$ ) as well as their respective frequency-magnitude distributions (Figure 4.8c). Panels a and b do not reveal striking differences in the rate of seismicity between compartments, with the exception of an increased rate of events in the northeast compartment (coral-colored) relative to the others at the end of 2012, which will be investigated in Section 4.5. However, panel c reveals a significant variation in $b$-value between compartments. While the western and northeastern compartments have $b$-values of $0.99 \pm 0.03$ and $0.88 \pm 0.04$, respectively, the $b$-value in the southeast compartment is markedly higher (1.18 \pm 0.04$)$. Given that the southeast compartment is the closest to the main injection wells (RK20, RK23 and RK24), this may suggest that the $b$-value at Rotokawa is proportional to pore-fluid pressure, which is most elevated near injection wells. A similar, but stronger, effect was observed by Bachmann et al. (2012) at the Basel injection site in Switzerland, who found $b$-values $>2.0$ near the injection interval, albeit for wellhead pressures (30 MPa) an order of magnitude higher than observed at Rotokawa $(<2 \mathrm{MPa}$ ) Häring et al., 2008).

To test the significance of the compartment $b$-value variations, we use the approach of Utsu (1999) where the probability that any two sub-catalogs come from the same population is given as Wiemer et al. (1998):

$$
P \approx \exp (-d A / 2-2)
$$

Here, $d A$ is the difference in the Akaike Information Criterion for the null hypothesis (i.e. both catalogs have the same $b$-value) and the hypothesis that their $b$-values are distinct such that: 

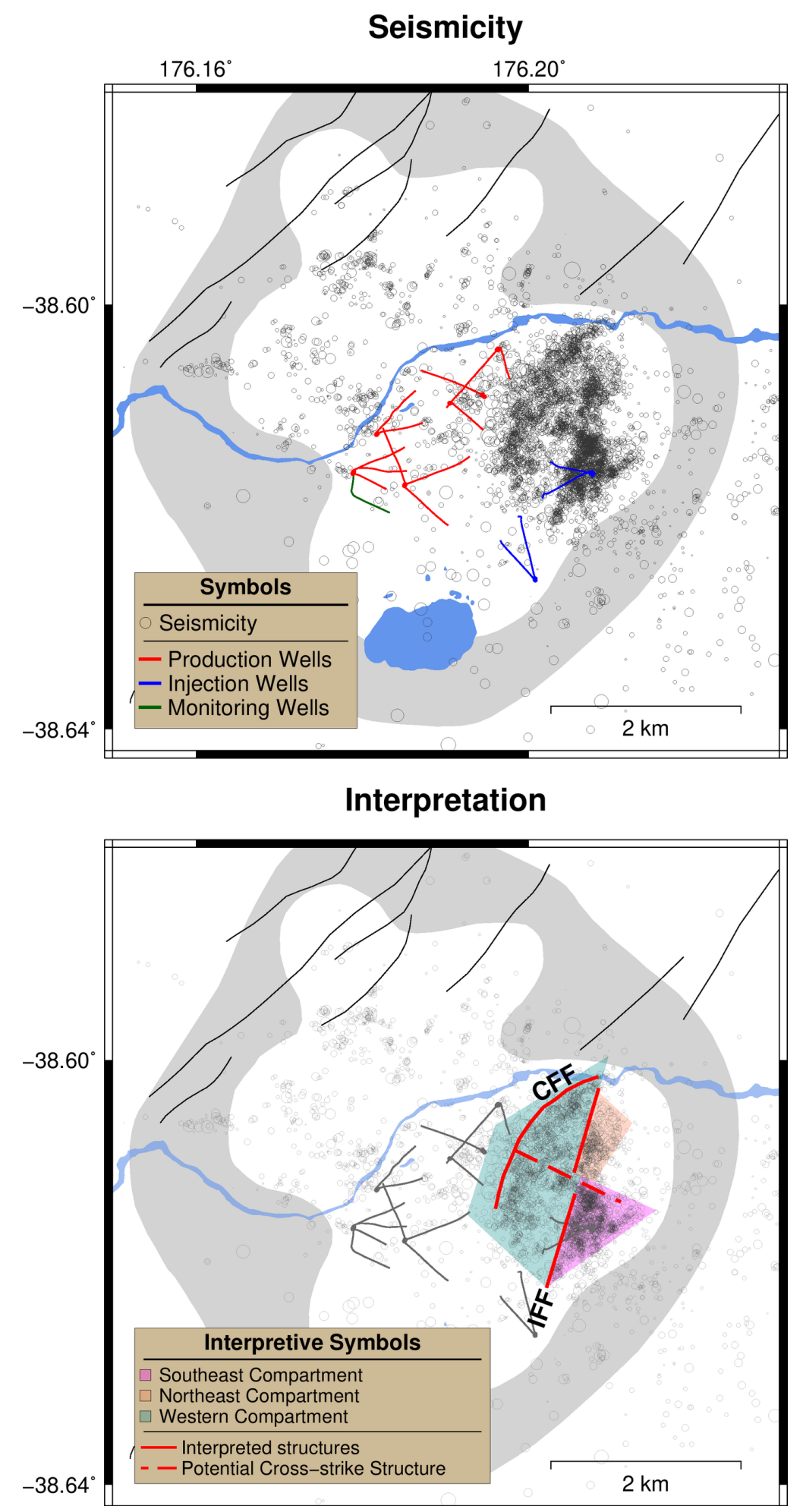

Figure 4.7: Seismicity at Rotokawa from 2012-2015, relocated with GrowClust. The top panel shows seismicity as black circles, scaled to each earthquake's magnitude (as in Figure 4.3). The bottom panel shows our interpretation of reservoir structure from the location of seismicity (red lines). These structures may define reservoir compartments (colored regions). The location and orientation of the Central Field Fault and Injection Field Fault (solid red) are well constrained from seismicity. In addition, the dotted red line indicates the location of a possible cross-strike structure north of the injection wells, which may contribute to further injection-field compartmentalization. 


$$
d A=-2 \ln N+2 N_{1} \ln N_{1}+N_{2} b_{1} / b_{2}+2 N_{2} \ln N_{1} b_{2} / b_{1}+N_{2}-2
$$

where $N_{1}$ and $N_{2}$ are the number of events in the two catalogs being compared, $N$ is $N_{1}+N_{2}$ and $b_{1}$ and $b_{2}$ are the $b$-values of the individual catalogs.

Using this method, we find that elevated $b$-value in the southeast compartment is significant at the $99.98 \%$ level or greater relative to both the western and northeastern compartments.

\subsection{Temporal variations in seismicity and injection parameters}

Characteristics of seismicity are used by geothermal operators to supplement more traditional, borehole-based pressure and temperature measurements for reservoir management. At Rotokawa, microseismicity has been used to constrain the location of major structures such as the $\mathrm{CFF}$ as well as the base of the reservoir (inferred from the cutoff in seismicity with depth). As outlined in Section 4.1.2, the Rotokawa reservoir behaved unexpectedly during specific periods in our dataset. We address these periods below and comment on the implications for processes occurring in the reservoir.

\subsubsection{RK24 injectivity decline}

Starting at the beginning of 2012, RK24, the largest injection well by percentage of injection at Rotokawa, began to suffer a decline in injectivity, meaning that it could no longer accept the same volume of fluid for a given wellhead pressure (Figure 4.9). This is a significant issue, because economical power plant operation requires a set enthalpy from a reservoir, which demands a set quantity of fluid to be extracted and injected. If a well cannot accept the fluid budgeted for it, that fluid must be shifted elsewhere, which can have negative implications for resource management. In the case of RK24, the fluid was shifted to both shallow injection well RK12 and deep injection well RK23, which injects into the reservoir on the opposite side of the IFF from RK24.

As the feedzones at RK24 (and RK20) are located to the west of the IFF and may therefore be better hydraulically connected to the western compartment (Figure 4.7), we expect seismicity in the western compartment to respond most readily to injection changes at these wells. Western compartment seismicity and relevant RK24 injection parameters are summarized in Figure 4.9. Injectivity begins to decline in the first half of 2012, after which Mercury made the decision to switch injection away from RK24. The switch can easily be seen as drops in flow rate, wellhead pressure and injectivity in July 2013 and again in July 2015. 

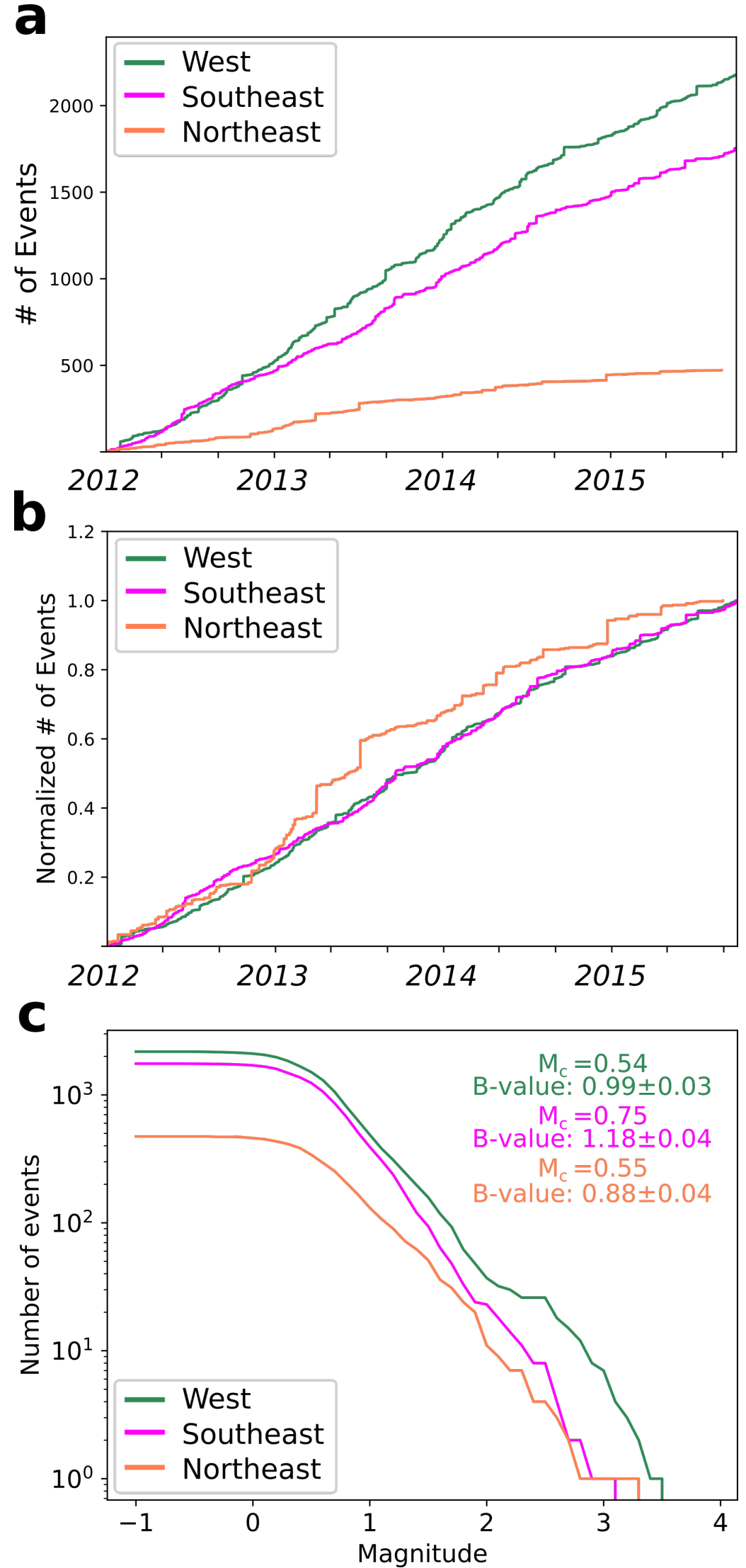

Figure 4.8: a) Total and b) normalized cumulative number of earthquakes in each of the three compartments defined in Figure 4.7. c) Frequency-magnitude distributions for each of the compartments. 

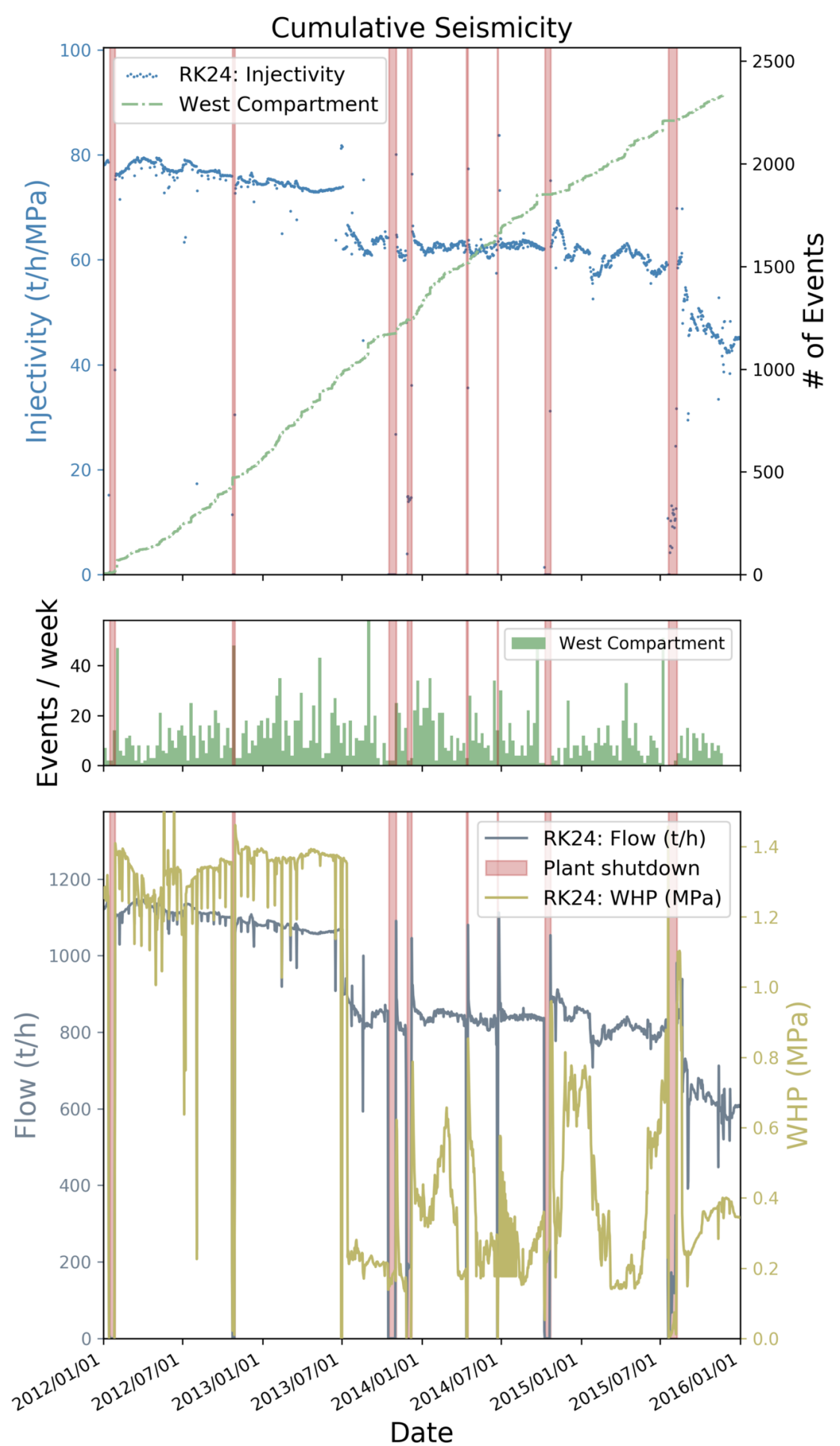

Figure 4.9: Seismicity in the western compartment compared to borehole parameters for well RK24. The top panel shows the cumulative number of events (green dotdashed) with injectivity at RK24 (blue). The middle panel shows the weekly rate of seismicity in the western compartment. The bottom panel shows both flow rate (dark gray) and wellhead pressure (yellow). Red shaded regions in all panels indicate the periods during which the power plants were shut down for maintenance, which have been recognized previously as periods of potentially heightened seismicity. 
The response of western compartment seismicity to the injectivity decline and subsequent decrease in flow at RK24 is subtle or nonexistent. In addition, the drop in WHP in July 2013 from $\sim 1.3$ to $0.2 \mathrm{MPa}$ does not produce the expected drop in the rate of seismicity. While the injectivity decline, and in particular the subsequent pressure drop might be expected to produce significant changes in the character of seismicity, there are a number of reasons why this may not be the case. The most significant complication to interpretation is that RK24 is not the only injector in this part of the field. Even if the pressure signals between RK23 and RK24 are isolated by the IFF, RK20 (on the same side of the fault as RK24) remained a significant injector throughout the dataset, injecting at a roughly constant rate of $600 \mathrm{t} / \mathrm{h}$ and a wellhead pressure of approximately 0.4 MPa. This may have provided enough pressure support in this section of the reservoir to continue to induce seismicity at close to previous rates. In addition, even as pressure had dropped at RK24, the injection rate was still $\sim 800$ t/h. As Sherburn et al. (2015) and Sewell et al. (2015b) have postulated, Rotokawa seismicity is likely affected more by stress changes induced by reservoir cooling than reservoir pressure increases, especially near the well. The results in Figure 4.9 support this view as seismicity seems insensitive to pressure perturbations of up to $1 \mathrm{MPa}$ at the wellhead, which are normally more than sufficient to induce seismicity Keranen and Weingarten, 2018; Stein, 1999).

\subsubsection{RK23 halt and restart}

Most of the excess injection displaced by the RK24 injectivity decline was accounted for by shallow injection into well RK12 (in the current production field) and deep injection into RK23. Prior to this, RK23 was being used as an injector for RGEN condensate until late 2012, when it was shut for 8 months (gray bar, Figure 4.10) before resuming injection, this time of NAP brine (Addison et al., 2017). In Figure 4.10, we plot characteristics of seismicity in the compartments that we interpret to be east of the IFF, where the pressure signal from RK23 is assumed to be the strongest. Similar to the western compartment, the rate of seismicity is mostly constant over time. Interestingly, the northeast compartment, furthest from RK23, experienced an increase in the rate of seismicity during the 8 months when no injection was occurring at the well, whereas the southeast compartment did not. This increased rate of seismicity is still significantly lower than in the compartment near the well, but it is unclear why seismicity further from the well would respond more strongly to a pressure perturbation than seismicity nearer to the well. 


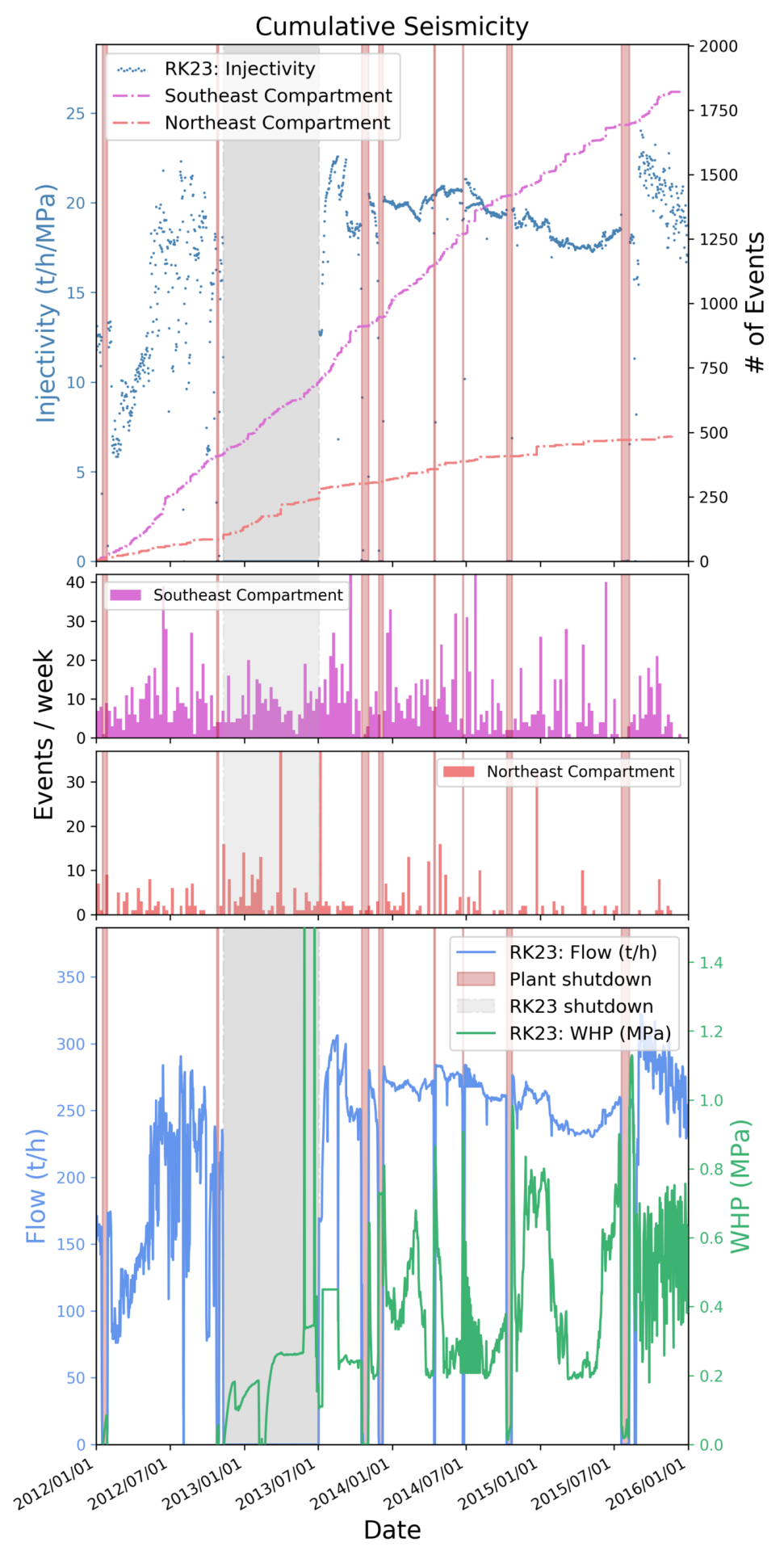

Figure 4.10: Seismicity in the eastern compartments compared to borehole parameters for RK23. The top panel shows the cumulative number of events (magenta and coral colored lines) with injectivity at RK23 (blue). The middle two panels show the weekly rate of seismicity in the southeastern and northeastern compartments, respectively. The bottom panel shows both flow rate (blue) and wellhead pressure (green). Red shaded regions in all panels indicate the periods during which the power plants were shut down for maintenance, which have been recognized previously as periods of potentially heightened seismicity. The period during which RK23 was shut is shaded in gray. 


\subsubsection{Aseismic injection: RK34 drilling and RK21/RK22}

One new well, RK34, was drilled at Rotokawa during our study period. Although RK34 drilling incurred full fluid losses at reservoir depths, there was no discernible response in seismicity near the well, or anywhere else in the reservoir. This behavior is distinct from the similar case of NM10 drilling in Ngatamariki the year before, which induced a significant number of events (Hopp et al., 2019, Chapter 3). However, aseismic injection is not uncommon at Rotokawa or Ngatamariki in general (Sewell et al., 2015b). For instance, drilling, and stimulation at well NM09 in Ngatamariki was largely aseismic, and injection into the southwestern injection wells, RK21/RK22 at Rotokawa has historically been aseismic (Hopp et al., 2019; Sewell et al., 2015b).

The case of RK21/RK22 is particularly puzzling, because RK21 was used as the dominant injection well from NAP startup (>1000 t/h) until 2011, but seismicity has never been observed in this section of the field (Sewell et al., 2015b). What's more, temperature and permeability in RK21/RK22 are highly similar to RK20/RK24. Why, then, does seismicity occur at one and not the other? Modeling at the Geysers geothermal fields has shown that density driven, downward flow can drive stabilization of the reservoir fracture network in regions where $\sigma_{1}$ is vertical (such as at Rotokawa) (Jeanne et al., 2015a). Although such an effect may help explain a lack of near-well seismicity in general, it does not explain the discrepancy between wells with such similar characteristics.

\subsection{Spatial $b$-value variations}

Figures 4.6 and 4.11 reveal a complex pattern of $b$ within the Rotokawa reservoir that defies simple explanation. The higher $b$-value of the southeastern compartment as a whole, shown in Figure 4.8c, is also discernible in Figure 4.6 (map view). However, $b$ is not uniform in space throughout any of the compartments. A profile of $b$-value for all compartments combined with distance from well RK23 or RK24 is shown in Figure 4.11 .

If we use the inferred IFF to divide the $b$-values into eastern and western compartments, on the basis that the pressure evolution on either side of the fault would be decoupled, the profiles take the shape of those in Figure 4.11 and $\mathrm{b}$. In the western compartment (Figure $4.11 \mathrm{~b}$ ), $b$ generally decays with distance from the well out to approximately $750 \mathrm{~m}$, as predicted by the geomechanical modeling of Bachmann et al. 2012), but increases from $750 \mathrm{~m}$ outwards. East of the IFF, mean $b$-value behavior is more complicated, varying between $\approx 1.0$ and 1.2 .

For all compartments, $b$-value also shows a depth dependence wherein the mean $b$ decreases from the surface to a minimum of $b \approx 1.0$ at $2750 \mathrm{~m}$ (roughly the depth of 

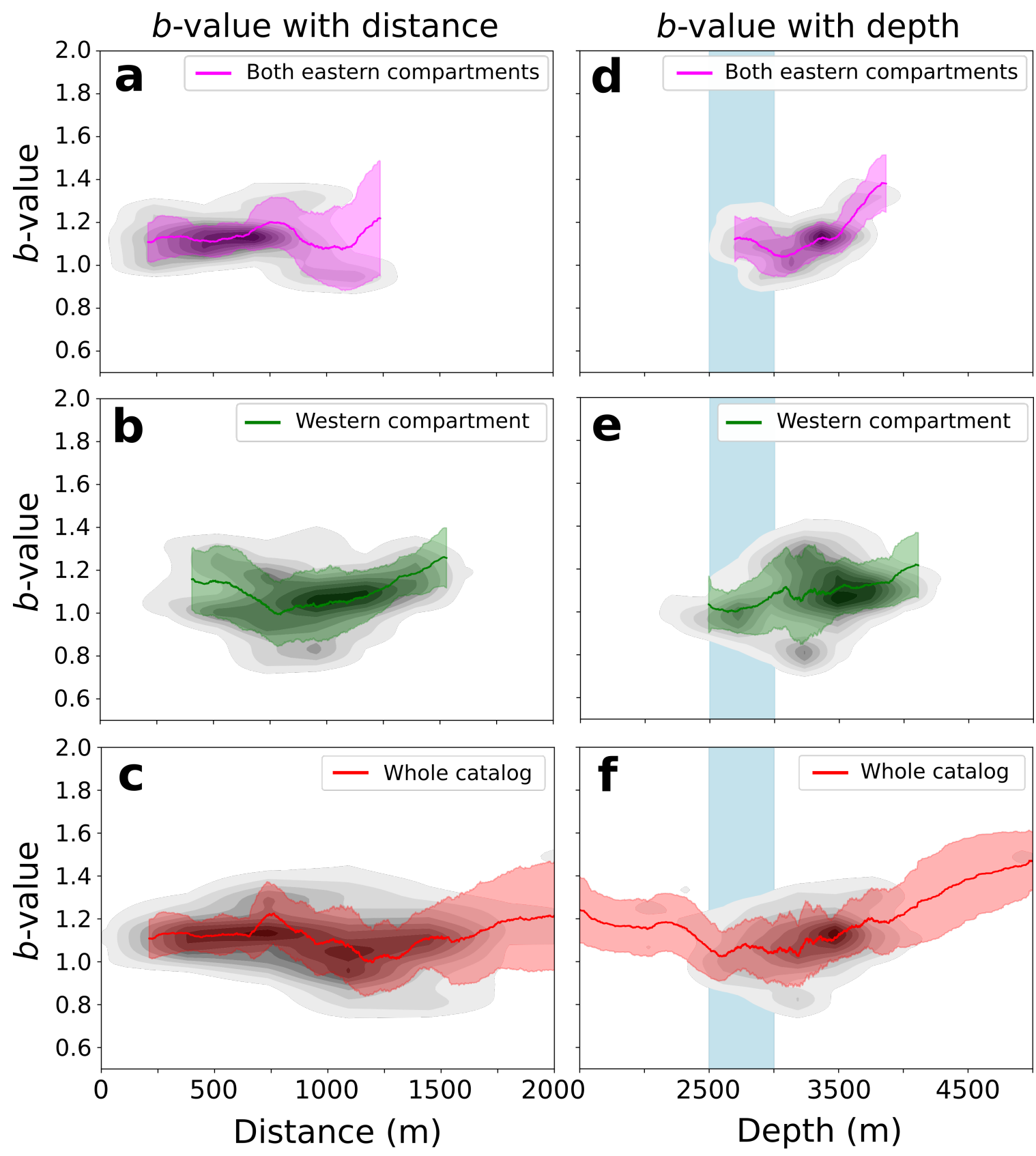

Figure 4.11: $b$-values with radius from the bottom of injection well RK23 (panels a and c) or RK24 (panel b). Panel a) shows the distance from RK23 to all events in both eastern compartments, combined. Panel b) shows the distance from well RK24 to all events in the western compartment. Panel c) shows the distance from RK23 to all events in the catalog. For all panels, the solid line shows the mean $b$ in a moving window of 200 events with the filled area showing one standard deviation. The statistics are plotted starting only once the window reaches a minimum of 200 events (moving from the left). Panels $\mathrm{d}$, e and $\mathrm{f}$ show the $b$-value distribution with depth for the catalogs in panels $\mathrm{a}, \mathrm{b}$ and $\mathrm{c}$ with the blue patches indicating the approximate depth of injection from wells RK23 and RK24.

injection) and then increases with depth until the base of seismicity at roughly $5 \mathrm{~km}$. This appears counter intuitive, given the currently-accepted reasoning that $b$-value is 
inversely proportional to differential stress, which generally increases with increasing overburden at depth (Schorlemmer et al., 2005). However, $b$-value has been shown to increase with depth in certain locations, especially in volcanic areas (e.g. Wiemer et al. 1998), perhaps owing to some combination of pervasive fracturing near intrusive bodies, high pore pressures, changing thermal gradients and an increase in host rock heterogeneity Schorlemmer et al., 2005; Wiemer et al., 1998; Warren and Latham, 1970). All of these characteristics are present at Rotokawa, which image logs and well cuttings have revealed to be a highly-fractured and geologically heterogeneous reservoir (Massiot et al., 2017; McNamara et al., 2016). However, very little is known about what lies beneath New Zealand geothermal fields, why they exist where they do or how they are fed (Wilson and Rowland, 2016). Evidence from drilling at Ngatamariki has shown that intrusive bodies do exist at depths $<3 \mathrm{~km}$ in the Taupō Volcanic Zone Chambefort et al. 2016) and geochemical evidence suggests a similar intrusive body may sit below Rotokawa (Winick et al., 2009). However, the true nature of the heat source is far from certain (Wilson and Rowland, 2016).

Unfortunately, it is difficult to make the case that geologic heterogeneity and the degree of fracturing increase with depth below the base of the reservoir, as the reservoir itself is already both heterogeneous and highly-fractured (Massiot et al., 2017; $M c N a$ mara et al., 2016). Also, pore-pressure perturbations originating at the injection wells decay with distance. This systematic decrease in $b$-value at reservoir depths may be a product of vertical contraction of the reservoir due to cooling, as suggested above. This would produce a preferential decrease in $\sigma_{1}\left(\approx \sigma_{V}\right.$ at Rotokawa), thereby decreasing the differential stress in the reservoir and counteracting the effect of pore-pressure buildup.

It is also possible that the depths presented for our catalog are systematically too deep, likely due to our lack of knowledge of the S-wave velocity structure in the field. Errors reported by bootstrap resampling of the input data used by GrowClust indicate an average horizontal uncertainty of $200 \mathrm{~m}$ and an average depth uncertainty of $278 \mathrm{~m}$. However, we relocated the catalog using only P-picks, and also using $V_{P} / V_{S}$ ratios of 1.86 and 2.0 (compared to $V_{P} / V_{S}=1.72$ presented here), all of which failed to change the depth dependence of $b$-value in the catalog (see Appendices 4.8.3). Once work is completed on a 3-D tomographic velocity model of the Ngatamariki and Rotokawa geothermal fields (by PhD student Steven Sewell), we may be better able to shed light on the depth uncertainties of seismicity at Rotokawa.

\subsection{Conclusions}

In this work, we analyze a four-year catalog of seismicity (2012-2015) for the Rotokawa geothermal field, corresponding to the four years immediately following the analysis of 
Sherburn et al. (2015) and Sewell et al. (2015b). While seismicity during these four years is confined to the injection field, our catalog is able to identify structures within this compartment, revealing previously unknown compartmentalization in this part of the reservoir. The locations presented are able to further constrain the location and orientation of the Central Field Fault, and for the first time define the location of the Injection Field Fault, previously mapped only through vertical well cutting offsets and temperature gradients between wells. In addition, we have identified a new structure, cutting across the dominant NE-SW structural grain. This structure may help explain the apparent pressure 'leak' between the injection field and central production compartment identified by tracer testing (Addison et al., 2017), where previously no pressure support was identified.

Finally, we have mapped the magnitude-frequency distribution (b-value) in the reservoir. The pattern revealed is complex and fails to conform to a simplified model wherein $b$ decays exponentially with distance from a pressure source Bachmann et al., 2012 ). However, $b$ is significant different between our inferred compartments. Specifically, $b$ is higher to the east of the Injection Field Fault, suggesting that pressure may not be diffusing as readily in this section of the injection field, allowing non-critically stressed fractures to fail more often than in other compartments. It is possible that $b$-mapping at fields elsewhere could help to identify areas of the reservoir with large pressure gradients or changes in the degree of fracturing, which might exhibit as changes in the seismic $b$-value. In general, we believe these and other similar uses of earthquake magnitude information are under-utilized as a tool for reservoir understanding and management.

\subsection{Appendices}

\subsection{1 $b$-value mapping parameters}

Figure 4.12 shows the pair-wise relationships between $b$-value, standard error and the volume occupied by the 300 events in each subcatalog mapped in Figures 4.6 and 4.11 . The diagonal plots show the distribution and mean of each parameter, the plots below the diagonal are density maps of the points shown in the plots above the diagonal. The upper plots also show the linear regression of the two variables being plotted. $b$ shows a strong colinear relationship with the standard error, much as it did with $M_{c}$ before we decided to enforce a constant $M_{c}$ for all catalogs. However, it shows only a weakly negative correlation with the volume occupied by the events in the catalog. This can be explained by a scale dependence of the $b$-value wherein a catalog occupying a larger volume encompasses larger structures than one occupying a smaller volume. The larger structures can accommodate larger slip patches when they fail, meaning larger 
magnitude events and a lower $b$-value. It should be noted, however, that this bias, while present, is not strong. The top right panel of Figure 4.12 shows that catalogs with the mean volume of $\sim 1.72 e 8 \mathrm{~m}^{3}$ (similar to sphere with a radius of $\sim 500 \mathrm{~m}$ ) have wide-ranging $b$-values $(\sim 0.8-1.5)$
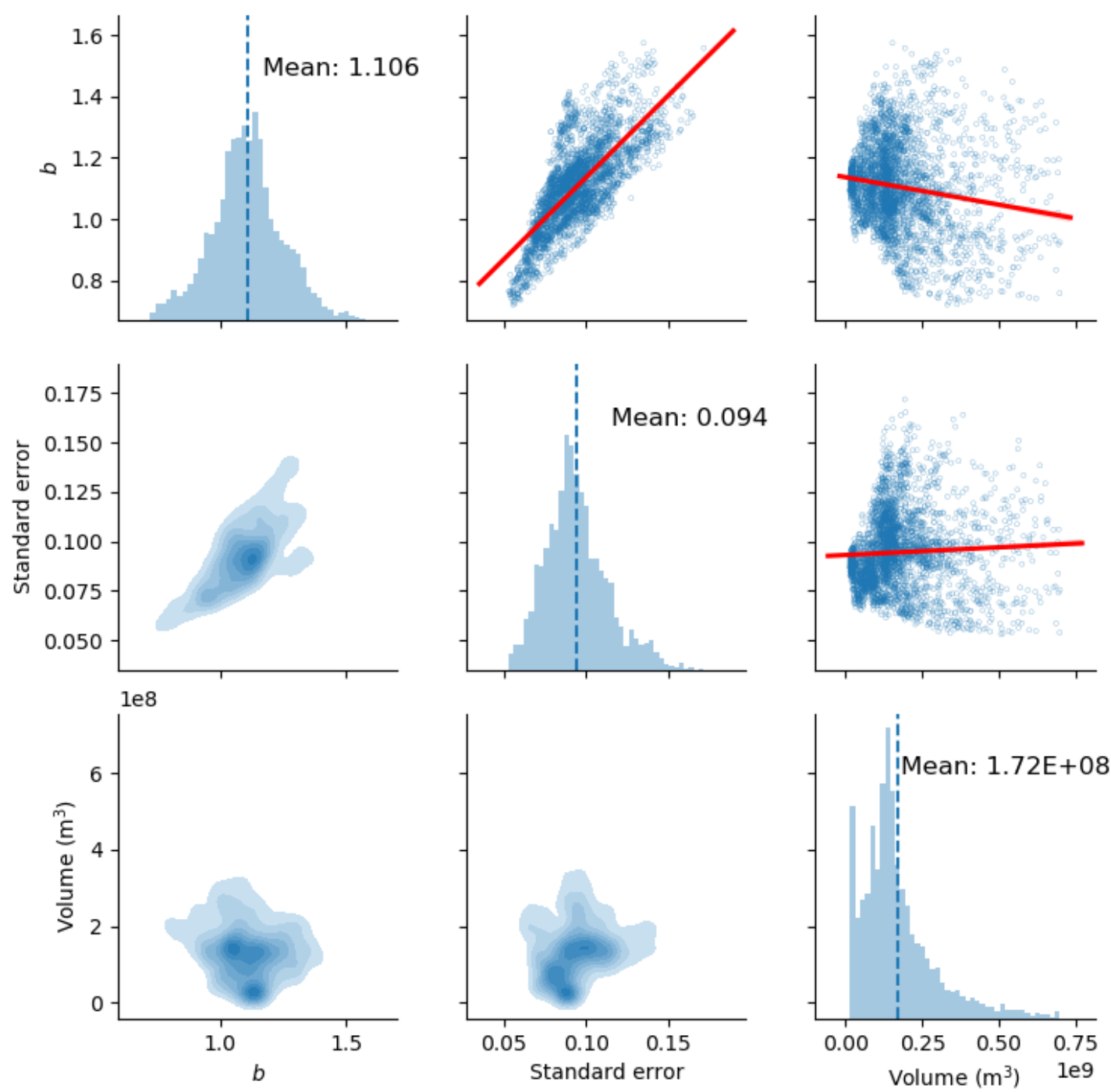

Figure 4.12: Pair plot of the results of the $b$-value mapping. Each of the four parameters ( $b$-value, standard error and the volume of the sub-catalog used in the calculation) is plotted against the others in a grid. The diagonal shows the 1-dimensional distribution of each parameter with the mean value noted in text and depicted as a dotted line. Because the grid is symmetrical about the diagonal, with the top and bottom triangles plotting the same data, we have chosen to plot the top as scatter plots with a linear regression plotted as a red line and the bottom as kernel density estimates (effectively heatmaps) in order to better show the point density. 


\subsection{2 'Swarm' activity}

Sewell et al. (2015b) and Sherburn et al. (2015) have observed periods of 'swarmlike' activity (defined as days with $>15$ events) at Rotokawa that may coincide with pressure perturbations caused by plant shutdown and startup periods. To determine if such behavior is also present in our catalog, we have plotted red bars in Figures 4.9. 4.10 and 4.13 indicating the times of plant shutdowns. The two shutdowns in 2012 (Figure 4.13) appear to be associated with 'swarm' events, defined here as days with more than 30 events due to the larger number of events in our catalog. However, the rest of the shutdowns appear to be anti-correlated with swarms periods with few events occurring while the plant is shutdown, as might be expected if we assume that events are purely induced by pore pressure increase.

The location of swarm activity in our catalog is much the same as was reported by Sewell et al. (2015b, Figure 5), with events occurring preferentially along the CFF, but with two other distinct clusters, one near RK23 and the other approximately 1 $\mathrm{km}$ to the north of RK23. The magnitude-frequency distribution of these events has a significantly lower $b$-value $(0.77 \pm 0.03)$ (Figure 4.15 than the catalog as a whole $(1.09 \pm 0.02)$. We interpret the relative abundance of large-magnitude events as an indication that swarm events are indeed occurring preferentially on the CFF, perhaps the only structure on which seismicity of $\mathrm{M}_{L}>3.0$ (Stein and Wysession, 2003, a rupture area of $\sim 0.1 \mathrm{~km}^{2}$ )) can occur within the field and which is optimally oriented for failure in the regional stress field.

\subsubsection{Location sensitivity to S-picks}

In order to better understand the robustness of our locations, and therefore the interpretations of the orientations of reservoir structures, we relocated the Rotokawa catalog without S-arrivals (Figure 4.16). Figure 4.17 shows the orientation of the Central Field and Injection Field Faults in map view as interpreted using the locations without S-arrivals. Although there does appear to be a slight shallowing of seismicity after removing S-picks, the interpretations of the orientations of the major structures are highly similar to those using the locations that incorporated both $\mathrm{P}$ - and S-phase picks. 


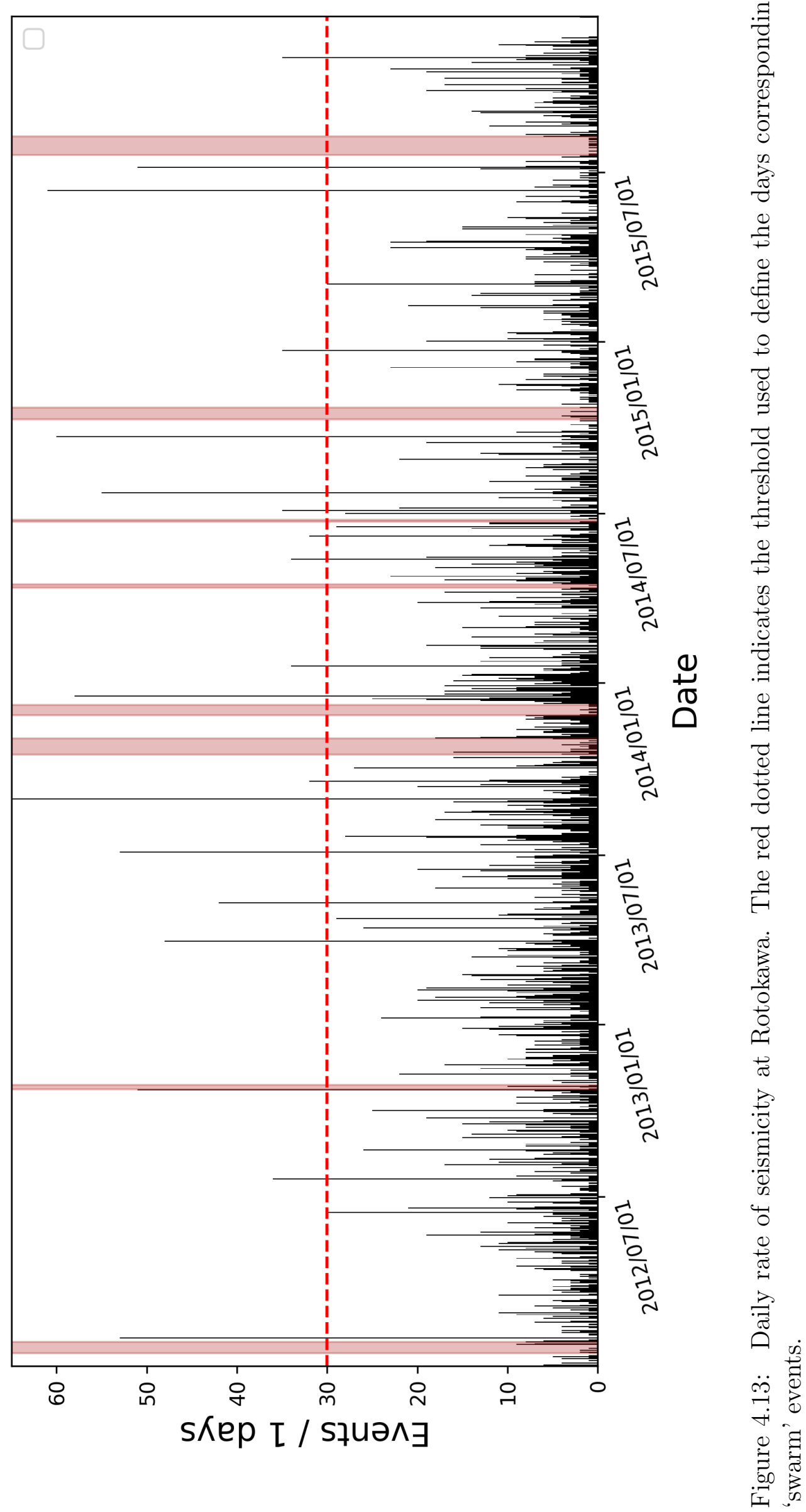



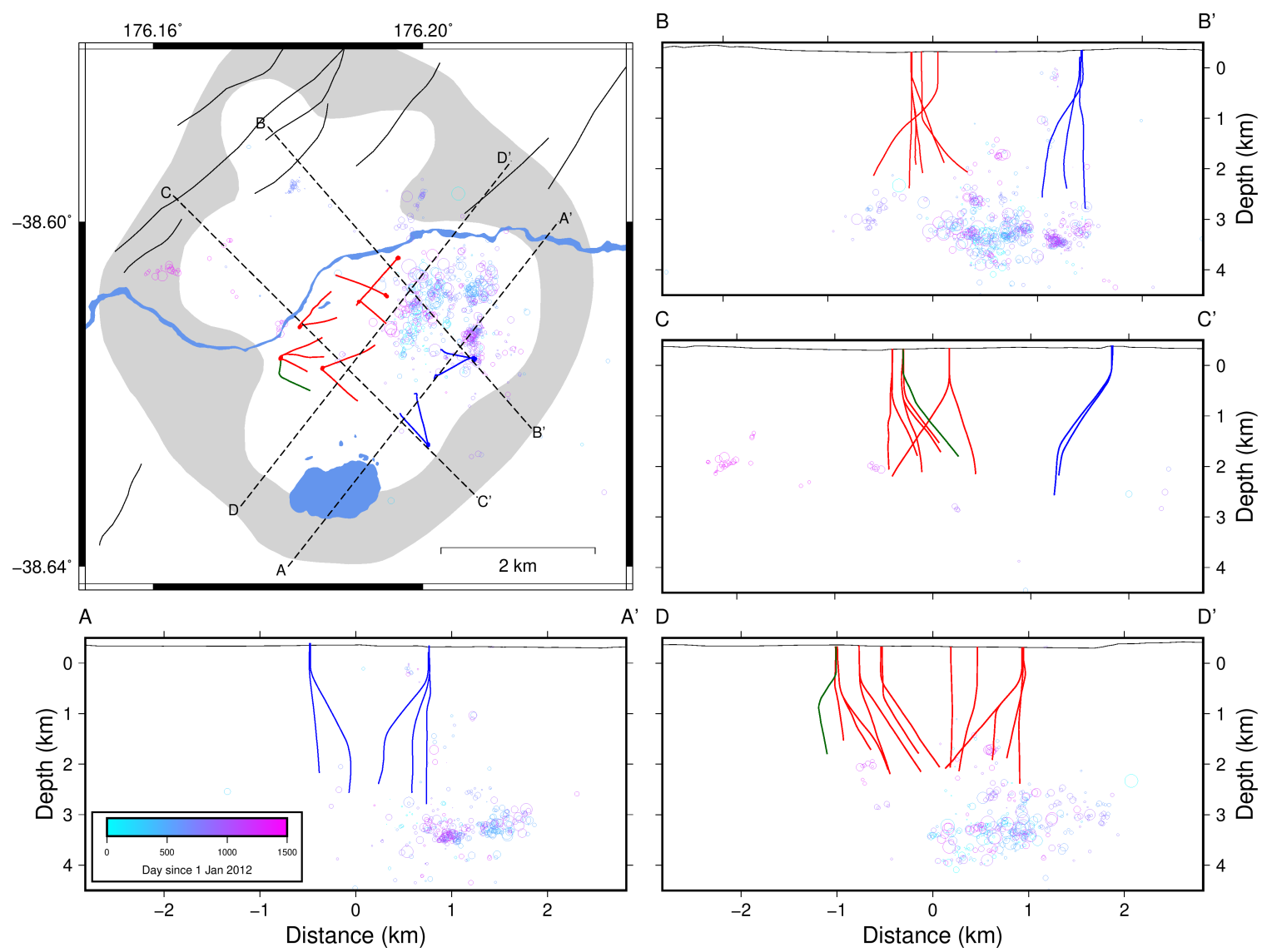

Figure 4.14: 'Swarm-like' events at Rotokawa. Swarms were defined as days on which more than 30 events occurred.

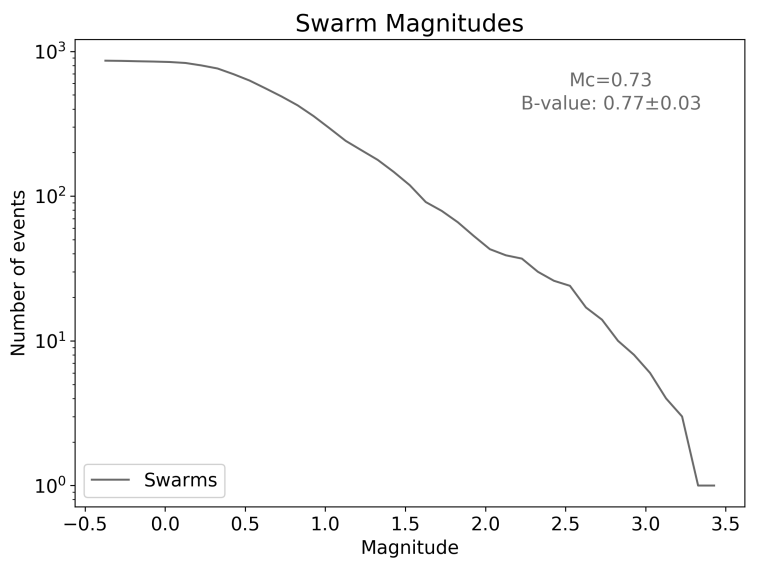

Figure 4.15: Frequency-magnitude distribution of events occurring on days of heightened seismicity (i.e. 'swarms'). 

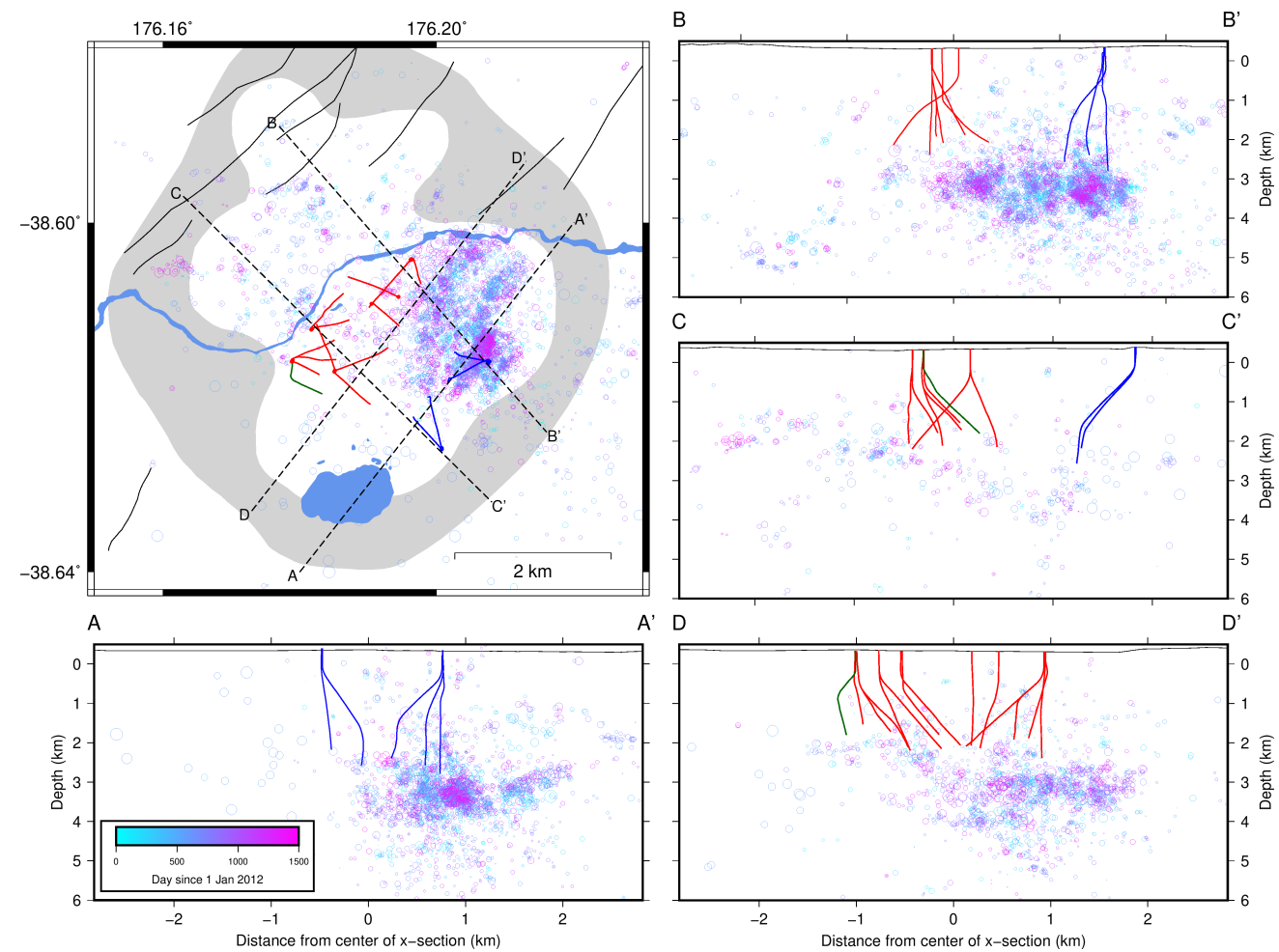

Figure 4.16: Seismicity at Rotokawa, located using only P-picks, for the years 2012 2015 colored by date of occurrence. Blue events occurred earlier in the dataset and pink events occurred later. Four cross sections are plotted to show the depth distribution of seismicity and their surface projections are shown in map view. 

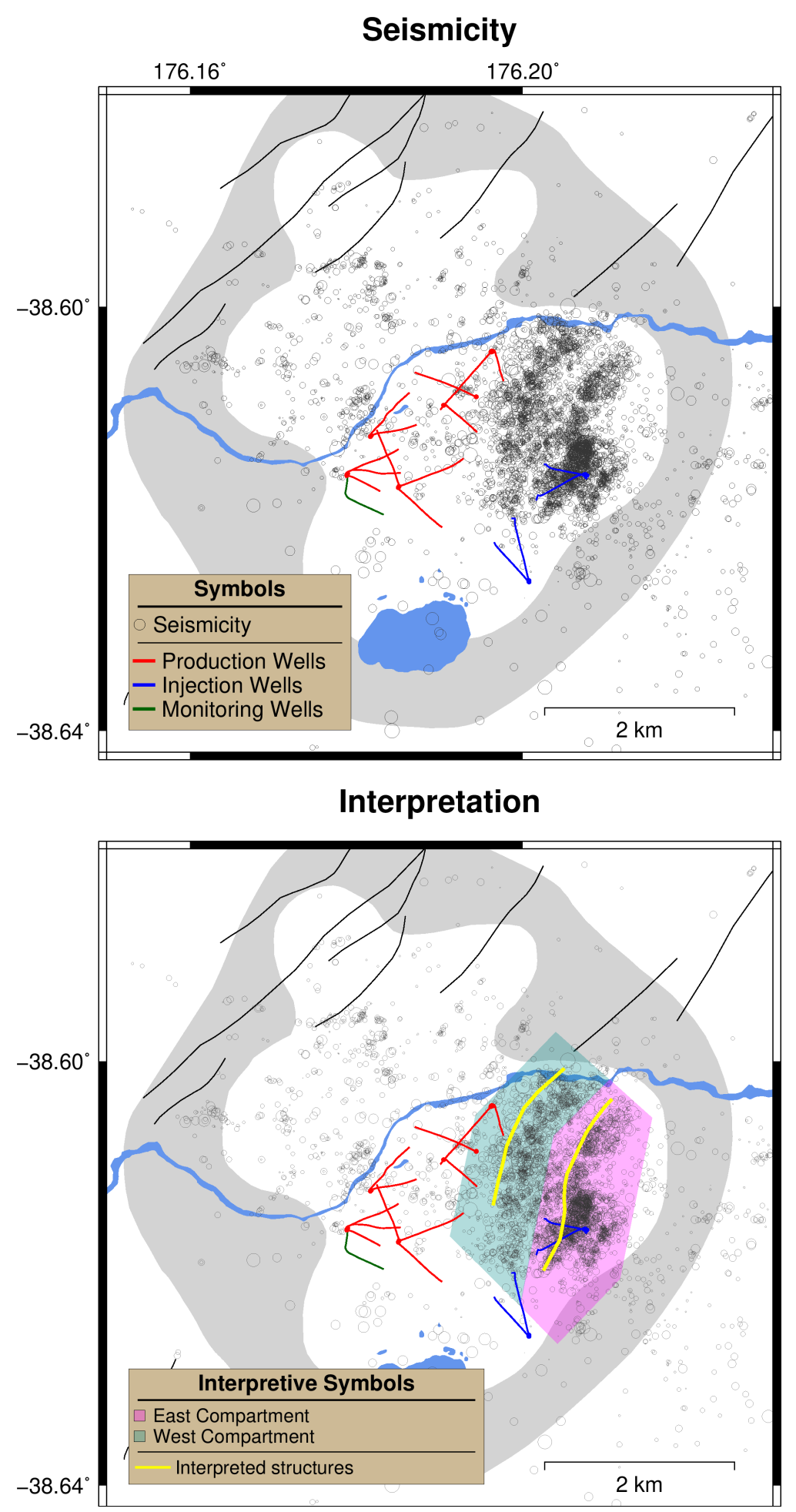

Figure 4.17: Seismicity at Rotokawa from 2012-2015, relocated with GrowClust using only P-picks. The top panel shows seismicity as black circles, scaled to each earthquake's magnitude (as in Figure 4.3). The bottom panel shows our interpretation of reservoir structure from the location of seismicity (yellow lines). These structures may define reservoir compartments (colored regions). The location and orientation of the Central Field Fault and Injection Field Fault are well constrained from seismicity. 


\title{
$5 \quad$ Focal mechanisms and stress state at Rotokawa and Ngatamariki
}

\begin{abstract}
Fluid injection and extraction activity has long been known to cause stress changes capable of inducing earthquakes. Typically, elevated pore-fluid pressure and extractioninduced poroelastic stress transfer are the causative mechanisms. However, at geothermal fields, thermal stresses induced by injecting cold water into a hot reservoir may play an under-appreciated and poorly understood role in changing the reservoir stress state. Here we present a catalog of 982 focal mechanisms calculated from P-wave first motion polarities for the Rotokawa and Ngatamariki geothermal fields in New Zealand. Mechanisms show the normal and strike-slip faulting expected in an active extensional setting. We cluster these mechanisms based on distance and time before inverting for the orientation of each principal component of stress as well as the stress field shape ratio, $\nu$. At the northern injection zone at Ngatamariki, $\sigma_{1}$ plunges $30^{\circ}$, indicating a strong deviation from the regional normal faulting regime $\left(90^{\circ}\right.$ plunge $)$, likely related to emplacement of a known intrusive body. At Rotokawa, $\nu$ drops from $\sim 0.9$ to 0.2 within six months of the reintroduction of a major injection well, before recovering to $\sim 0.9$ two years later. We interpret this change to be the result of anisotropic stress reduction due to thermal contraction of the reservoir. Simple, computationally fast stress inversions from focal mechanism observations can lead to further insights into the transient stress changes at geothermal reservoirs. In addition, if our interpretations are correct, stress ratio changes offer constraint on the shape of the injection-related cooled zone surrounding wells.
\end{abstract}

\subsection{Introduction}

The state of stress within exploited reservoirs is of paramount importance in dictating flow pathways for hydrocarbon, heat and/or geothermal brine (Zoback, 2010). Measuring the stress state in a reservoir located kilometers beneath the earth's surface is difficult and only possible in wells drilled to reservoir depth. The expense of drilling means that such wells are rare and intersect only small portions of a reservoir, leaving large volumes unsampled. As the reservoir stress state is known to vary laterally and 
with depth (Blake and Davatzes, 2011; Davidson et al., 2012; McNamara et al., 2015) on a scale of 100s of meters, stress variations likely go unnoticed away from wells.

When a reservoir is seismically active, earthquakes represent a manifestation of both fault/fracture orientation and the stress state that enabled slip on these fractures. Inversion of earthquake focal mechanisms can therefore be used to improve the stress picture in undrilled areas of the reservoir and can also provide information about the orientation of the fractures that host reservoir permeability.

The Ngatamariki and Rotokawa geothermal fields are high-temperature $\left(\sim 300^{\circ}\right.$ C), liquid-dominated geothermal reservoirs in the central Taupō Volcanic Zone (TVZ) of New Zealand (Figure 5.1), a zone of active extension and high-volume volcanism (Wilson et al., 1995). Both fields are hosted in a thick succession of rhyolitic and andesitic volcanic flows, tuffs and ignimbrites with ages of $<2 \mathrm{Ma}$ (Wilson et al., 1995; Wilson and Rowland, 2016). Rotokawa has been used for power production since 1997 and Ngatamariki since 2012, between them producing roughly $220 \mathrm{MWe}$.

In this work we present a catalog of earthquake focal mechanism solutions for both fields and use these catalogs to invert for the stress state. We attempt to address the resulting stress variations in space and time and comment on the potential relationships between power production (specifically fluid injection) and stress tensor variation. To our knowledge, this is the first study to use focal mechanism inversion to study stress state at a New Zealand geothermal field. However, the tectonic setting in the TVZ is comparable to that at large geothermal fields elsewhere (e.g. The Geysers in northern California, USA) where extensive stress studies have been conducted using both high-quality focal mechanism inversions and coupled thermo-hydro-mechanical numerical simulations (e.g. Martínez-Garzón et al., 2013, Boyle and Zoback, 2014, Jeanne et al. 2015a). We refer to these studies to provide context for our results and suggest mechanisms for stress change in geothermal reservoirs in general.

\subsubsection{Stress changes at geothermal reservoirs}

In the last three decades, a number of workers have attempted to characterize the state of stress in geothermal reservoirs and determine the potential changes related to fluid injection and extraction activities Oppenheimer, 1986, Feng and Lees, 1998; Sasaki and Kaieda, 2002; Bohnhoff et al., 2004; Martínez-Garzón et al., 2013; Boyle and Zoback, 2014, Martínez-Garzón et al., 2014, 2017). Although some of these studies were hampered by small amounts of data, the more recent works (specifically MartinezGarzón et al., 2013; Boyle and Zoback, 2014; Martínez-Garzón et al., 2014, 2017) at The Geysers geothermal field in California were able to make use of large datasets of high-precision, well-constrained focal mechanisms ( $n>6000$ events) to invert for the principal stress axes in time and space. 


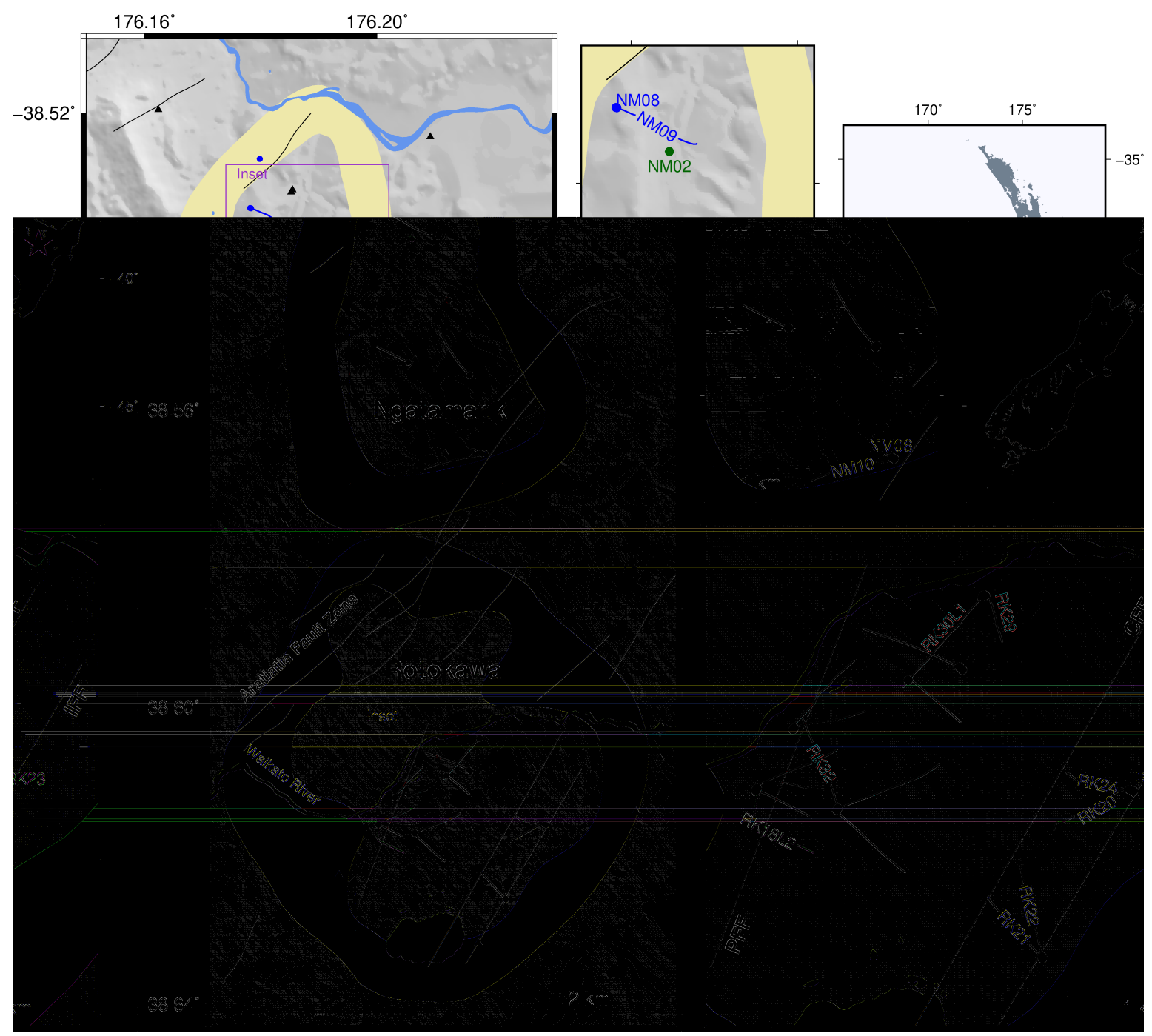

Figure 5.1: Overview map of the Ngatamariki and Rotokawa geothermal fields. Yellow polygons show the extent of each geothermal resource as defined by resistivity surveys (Risk, 2000; Boseley et al., 2010), black lines are mapped faults, and triangles are seismic stations used for this study. Within the fields, blue, red and green lines show the surface projection of injection, production and monitoring wells, respectively, with a circle indicating the location of the wellhead. Boreholes mentioned in the text are labeled in the inset panels. At Rotokawa, the modeled surface traces of the Production Field Fault (PFF), Central Field Fault (CFF) and Injection Field Fault (IFF) are plotted as dotted lines.

Although starting from the same underlying dataset, Martínez-Garzón et al. (2013) and Boyle and Zoback (2014) came to different conclusions from their stress inversion results. Boyle and Zoback (2014) concluded that there was no discernible spatial deviation in the stress field related to injection/extraction activities at The Geysers, based on their observation that $\mathrm{S}_{\text {Hmax }}$ was consistent inside and outside of the reservoir, as well as at different depth intervals and spatial grid sizes. In contrast, Martínez-Garzón et al. (2013) determined that The Geysers' stress state is normal/transtensional at reservoir depths but strike-slip/transtensional above and below. This is due to an 
apparent swapping of $\sigma_{1}$ and $\sigma_{2}$ axes at reservoir depths, leaving $\mathrm{S}_{\text {Hmax }}$ unchanged $\left(\mathrm{N} 15^{\circ} \mathrm{E}\right)$. Although Boyle and Zoback (2014) concluded that power production activities had no effect on the stress state of the reservoir, their observations are consistent with those of Martinez-Garzón et al. (2013), in that both show $\mathrm{S}_{\text {Hmax }}$ is unchanged throughout The Geysers.

Thermo-hydro-mechanical modeling of the stress response to injection at the Geysers (Jeanne et al., 2014, 2015a) supports the interpretation of Martínez-Garzón et al. (2013). They show that thermal contraction of the host rock at reservoir depths causes a variation in the dip of $\sigma_{1}$ at and below the depth of injection (Jeanne et al., 2015a). This is because the injected fluid, which is much denser than the hot reservoir fluid, 'sinks' upon entering the reservoir. This effect causes the cooled volume of the reservoir to take on a shape that is longer in the $\mathrm{z}$ (depth) direction than in the $\mathrm{x}$ or $\mathrm{y}$ direction, thereby reducing vertical stress more than the horizontal stresses. In their model, since $\sigma_{1} \approx \sigma_{V}$ at The Geysers (normal faulting regime), cooling reduction of $\sigma_{1}$ actually caused $\sigma_{1}$ and $\sigma_{2}\left(\mathrm{~S}_{\text {Hmax }}\right)$ to exchange orientation after 7-8 months of injection, artificially inducing a strike-slip faulting regime within the reservoir Jeanne et al., 2015a). No such effect was observed when the same models were run in the absence of thermoelastic effects, indicating that such dramatic stress rotations are unique to injections where the temperature contrast between the injected fluid and the reservoir is high (Jeanne et al., 2015a).

These results likely apply directly to the development of the Ngatamariki and Rotokawa reservoirs, where the stress state and natural-state reservoir temperature are similar to The Geysers.

\subsubsection{Fractures and stress state at Rotokawa and Ngatamariki}

At a reservoir scale, structure at Ngatamariki and Rotokawa mirrors the NE-SWstriking trend of extensional faulting observed across the Taupō Volcanic Zone. These NW- and SE-dipping antithetic structures accommodate the $\sim 5-15 \mathrm{~mm} / \mathrm{y}$ of NWSE regional extension (Rowland and Sibson, 2004, Wallace, 2004). The only major structure to intersect both the Ngatamariki and Rotokawa reservoirs is the Aratiatia Fault Zone (Figure 5.1). However, a number of other NE-SW structures of $>1 \mathrm{~km}$ in length have been inferred to exist within the fields Wallis et al., 2013; Chambefort et al. 2014). Within the production and injection fields at Rotokawa, three major faults have been identified from geologic modeling of vertical offsets of well cuttings (Wallis et al., 2013). From NW to SE these are the Production Field Fault (PFF), Central Field Fault (CFF) and Injection Field Fault IFF (Figure 5.1).

If we consider the reservoirs at a scale of $1-100$ s of meters, the orientation and extent of fracturing is mainly known through borehole image logs (see Section 5.2). At this scale, fracturing in the reservoirs exhibits the same NE-SW trend as the regional-scale 
faults. However, between wells and across both fields, the dominant dip of fracturing varies, likely controlled by the dip direction of the nearest large fault zone (McNamara et al., 2015).

The TVZ is a zone of active extensional tectonics, with a stress state described by $\sigma_{1} \approx \sigma_{V}$ and $\mathrm{S}_{H \max }$ trending NE-SW (Townend et al., 2012). Inversion of earthquake focal mechanisms has estimated the direction of $S_{H \max }$ in the central TVZ to have an azimuth of $\sim 052-058^{\circ}$ Hurst et al., 2002, 2008). Other estimates of the direction of $\mathrm{S}_{\text {Hmax }}$ have been made from the orientation of drilling induced tensile fractures and other induced structures observed in borehole image logs at Rotokawa McNamara et al., 2015). For wells RK18L2, RK32 and RK30L1, estimates of $\mathrm{S}_{\text {Hmax }}$ range from 025-049 ${ }^{\circ}$ McNamara et al., 2015). These orientations are also consistent with shearwave fast polarization directions measured by Mroczek et al. (2019) and Mroczek (2017). Davidson et al. (2012) attempted to estimate the magnitudes of the principal stress components at Rotokawa but were hampered by a number of complexities with the data, including complex lateral variations in the overburden and a lack of observed borehole breakout (McNamara et al., 2015). They were able to demonstrate that $\sigma_{V}$ and $\mathrm{S}_{H \min }\left(\sigma_{3}\right)$ at reservoir depths $(\sim 1100 \mathrm{~m} \mathrm{bsl})$ in Rotokawa vary laterally by up to $5 \mathrm{MPa}$ over scales of less than a kilometer, similar to results presented for the Coso geothermal field in California (Davidson et al., 2012; Blake and Davatzes, 2011).

\subsection{Data and methods}

\subsubsection{Earthquake catalog}

The earthquake catalog analyzed here was provided by GNS Science under contract with Mercury NZ Ltd. Earthquake detection and location were conducted automatically by GNS Science using the SeisComP3 software package (Weber et al., 2007). We then revised the entire catalog manually, adjusting arrival times where warranted and picking $\mathrm{P}$-wave first arrival polarities where possible. After removing all events with fewer than five polarity picks, 982 events remained. Finally, we relocated the entire catalog using the double-difference relocation program GrowClust Trugman and Shearer, 2017).

\subsubsection{Focal mechanism determination}

To calculate the focal mechanism solutions for this study, we used the Bayesian approach of Walsh et al. (2009), which allows us to incorporate known uncertainties of the input parameters, specifically in hypocentral location and polarity picking error. Solutions were calculated using only manually-picked $\mathrm{P}$-wave polarities, so long as a minimum of five picks were made per event. We calculated a total of 205 focal mecha- 
nism solutions at Ngatamariki and 777 at Rotokawa (Figures 5.2 and 5.3). There are a median of seven polarity picks per event and an average standard deviation of the strike/dip/rake of $31.4^{\circ}$.

As expected in the extensional TVZ, focal mechanisms at both fields show predominantly oblique normal faulting with subvertical P-axes. B-axis plunges are well distributed at Ngatamariki, showing no preference between pure normal and pure strikeslip faulting, whereas at Rotokawa, faulting is mostly normal with a minor oblique component (Figure 5.4). The mode of faulting at both fields appears to be unrelated to the hypocentral depth (Figure 5.4).

\subsection{3 kmeans clustering}

In order to investigate the spatial variation in the stress parameters at Rotokawa and Ngatamariki, we employ a 'kmeans' clustering technique (Hartigan, 1975), based on the Euclidian distances between all event pairs in the catalog. It is well known that the 'kmeans' algorithm does not yield a unique solution to this clustering problem (as noted by Townend et al., 2012), but visual inspection of the results shows that no clusters violate logical divisions in the hypocenter distribution (across inferred faults, for example). We therefore have no reason to suspect that any particular cluster samples multiple stress regimes, although this cannot be ruled out.

We divide the focal mechanisms at Ngatamariki (Figures 5.2) into $k=10$ clusters and at Rotokawa (Figure 5.3 into $k=30$ clusters, retaining only those clusters with 20 events or more. We performed the clustering over a range of $k$ values $(k=5-30$ at Ngatamariki; $k=18-60$ at Rotokawa) before selecting the value that maximized the number of groups at either field.

\subsubsection{Stress inversion}

For each of the clusters established above, we inverted for the three principal stress axes $\left(\sigma_{1,2,3}\right)$ and the stress ratio, $\nu$ :

$$
\nu=\frac{\sigma_{1}-\sigma_{2}}{\sigma_{1}-\sigma_{3}}
$$

using the approach of Arnold and Townend (2007). This approach allows us to readily incorporate the uncertainties in the focal mechanism parameters (strike/dip/rake) calculated using the approach detailed above and outputs a probability density function of $\sigma_{1,2,3}$ and $\nu$. These can then be incorporated into the transformation of Lund and Townend (2007), which returns an estimate of $\mathrm{S}_{\text {Hmax }}$ for cases in which one principal stress is not vertical. 

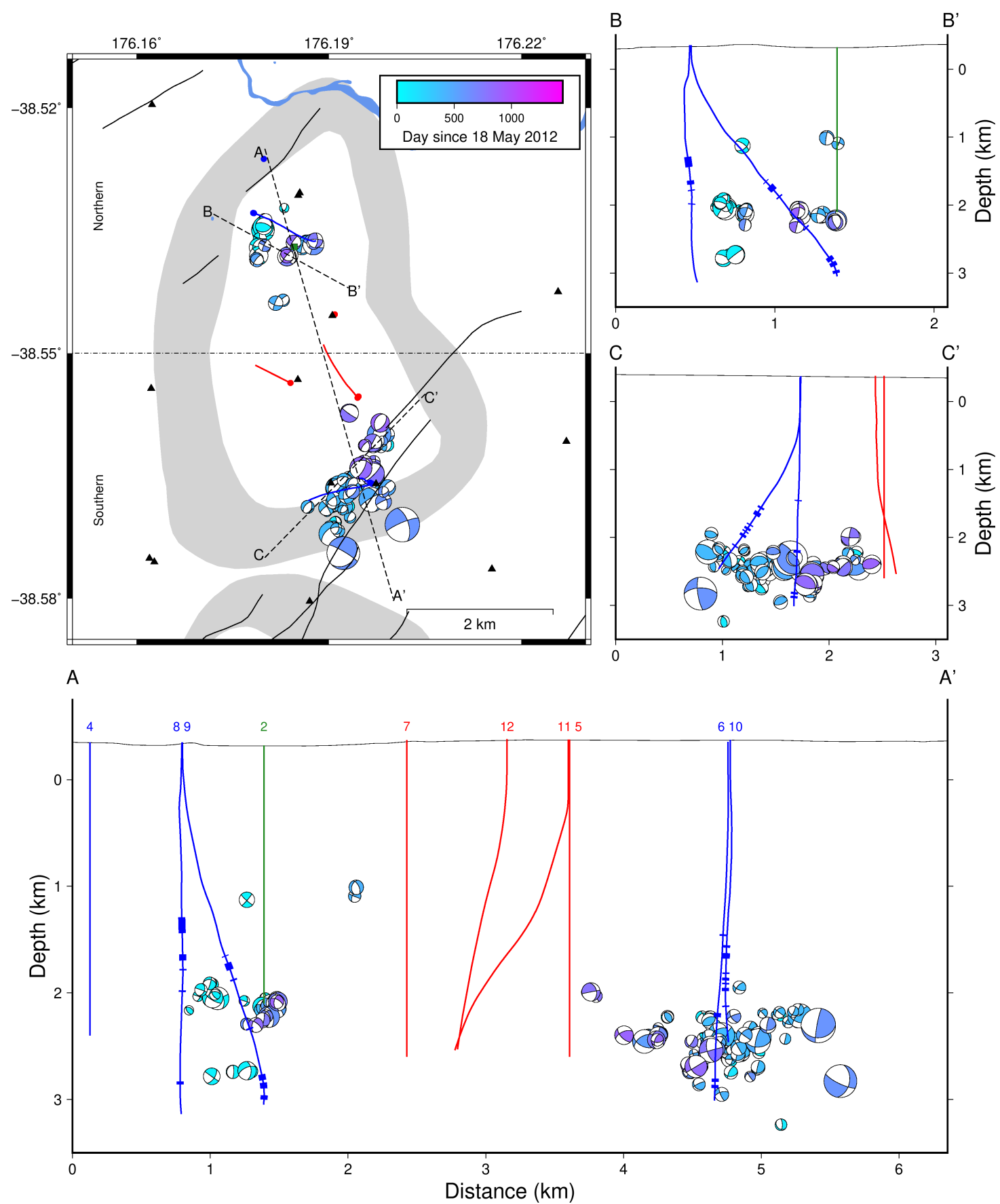

Figure 5.2: All calculated focal mechanisms for Ngatamariki from May 2012 until November 2015. Grey polygons show the resistivity boundaries for Ngatamariki (to the north) and Rotokawa (to the south). Black lines indicate active faults from the GNS Active Fault Database (Langridge et al., 2016). Red, blue and green lines indicate production, injection and monitoring wells. Focal mechanisms are colored by the date of occurrence, with earlier events colored blue and later events colored pink. Focal mechanisms have been reprojected in each cross-section view to show "backhemisphere" projections (i.e. the hemisphere "behind" the panel). Each cross section shows only the events within $1.5 \mathrm{~km}$ of the plane. Black triangles show the locations of seismic stations. 


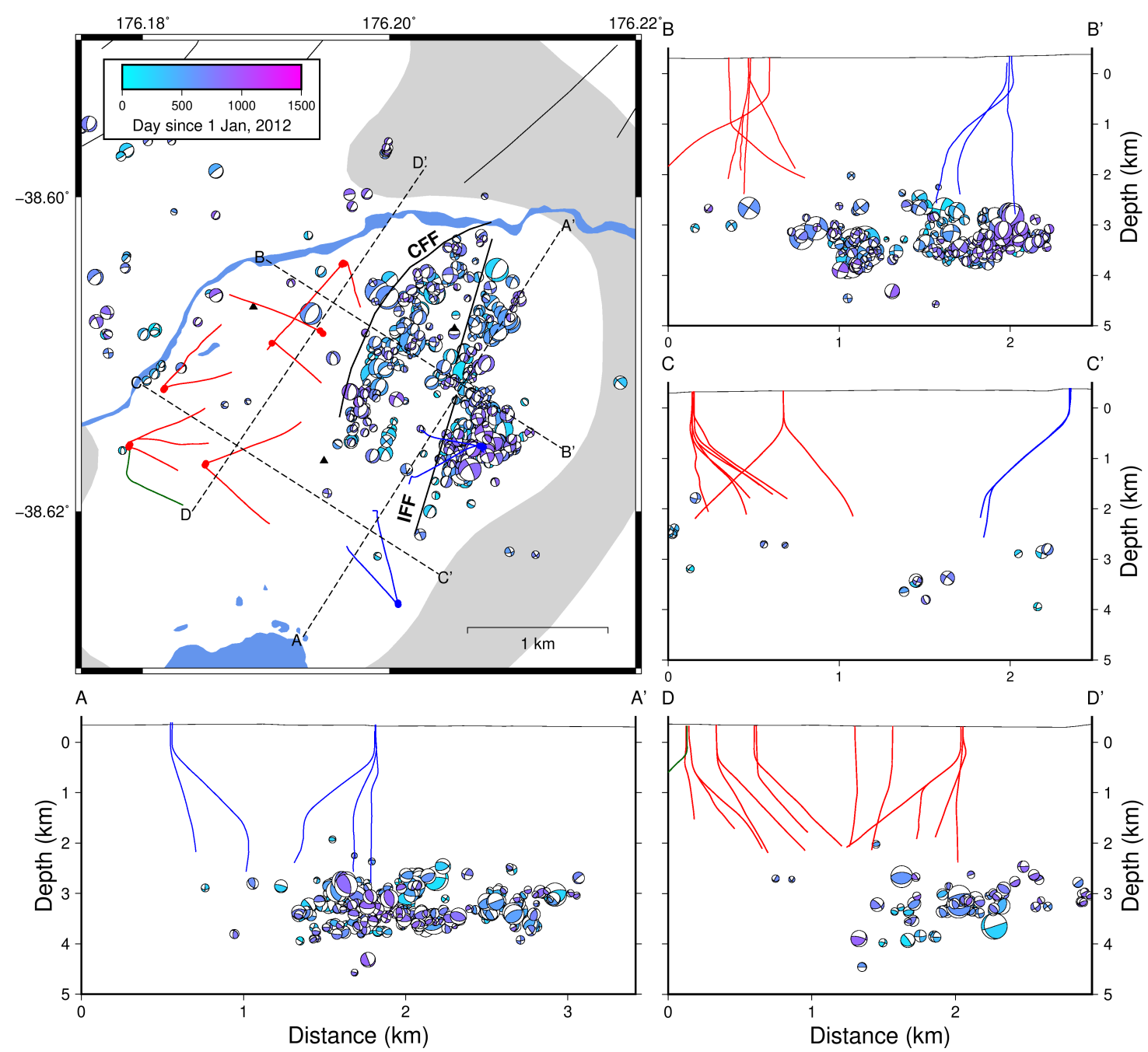

Figure 5.3: All calculated focal mechanisms for Rotokawa from May 2012 until November 2015. The grey polygon shows the resistivity boundaries for Rotokawa. Black lines indicate active faults from the GNS Active Fault Database (Langridge et al. 2016 ) with the inferred orientation of the \FF and CFF drawn in bold and labeled. Red lines indicate production wells, blue lines indicate injection wells and the green line is a reservoir monitoring well. Focal mechanisms are colored by the date of occurrence, with earlier events colored blue and later events colored pink. Focal mechanisms have been reprojected in each cross-section view to show "back-hemisphere" projections (i.e. the hemisphere "behind" the panel). Each cross section shows only the events within $1.5 \mathrm{~km}$ of the plane. Black triangles show the locations of seismic stations. 


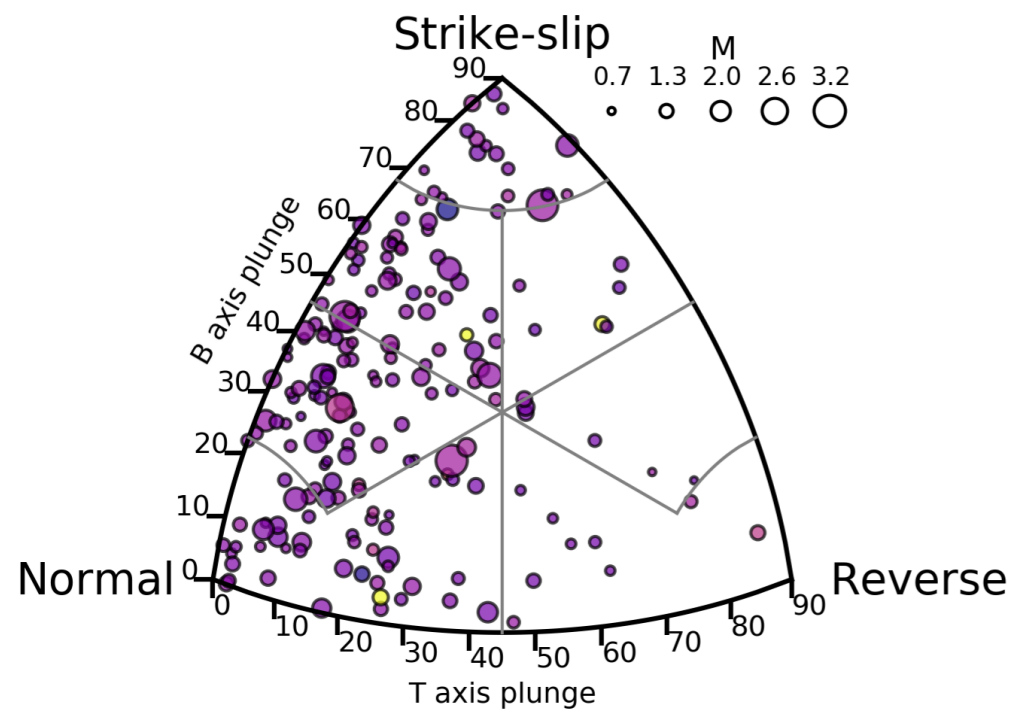

$-1.4$

2.0

$-2.7$

Ngatamariki Mechanisms

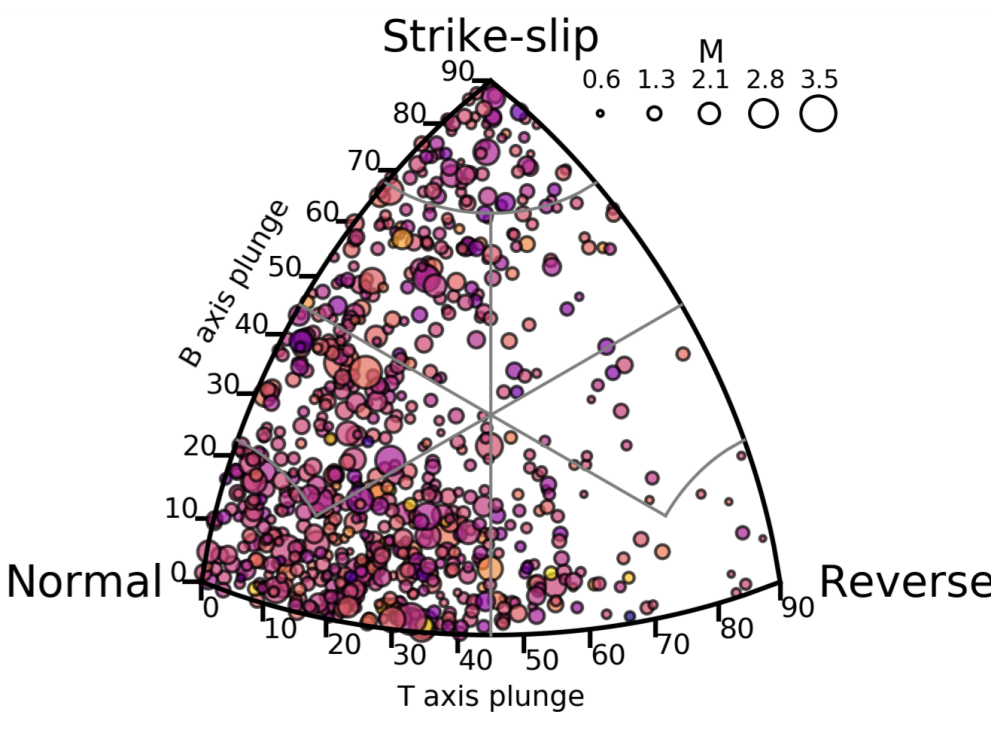

\section{Rotokawa Mechanisms}

Figure 5.4: Kaverina diagrams (Kaverina et al., 1996; Álvarez-Gómez, 2014) showing the kinematics for all focal mechanisms at Ngatamariki (top) and Rotokawa (bottom) based on the plunge of the B (null) and T-axes for each event. Circles are scaled by magnitude and colored by depth.

\subsection{Results}

\subsubsection{Stress inversions}

Ngatamariki

At Ngatamariki, kmeans clustering for $k=10$ yielded five clusters of 20 events or greater (Figure 5.5), two in the north and three in the south. In the north, Cluster 2 is located 
nearest to injection well NM08 and contains mostly events that occurred during NM08 stimulation (Hopp et al., 2019, Chapter 3), while Cluster 6 includes events occurring adjacent to injection well NM09 (Figure 5.5).

The southern clusters, (4, 1 and 9 from the SW to NE, respectively), run along the strike of the Aratiatia Fault Zone with Cluster 1 centered at vertical injection well NM06 (Figure 5.5).

Hopp et al. (2019, Chapter 3) performed stress inversions at Ngatamariki on a subset of the focal mechanisms analyzed here. The events analyzed occurred during the stimulation and completion testing of wells NM08, NM09 and NM10 in 2012-2013. They found the stress state in southern Ngatamariki to be normal, with $\sigma_{1} \approx \sigma_{V}$ and $\mathrm{S}_{\text {Hmax }}$ trending NE-SW at $\sim 045^{\circ}$. However, in the northern injection zone they found the stress regime to be inconsistent with regional normal faulting. There, $\sigma_{1}$ dipped to the $\mathrm{NE}$ at $\sim 30^{\circ}$ and $\sigma_{2} / \sigma_{3}$ formed a girdle, indicating a high stress ratio $(\nu>0.8)$, where their respective orientations were difficult to distinguish. The discrepancy between the stress states in the northern and southern injection fields was attributed to the emplacement of a tonalite intrusive body in northern Ngatamariki, which may have significantly deviated the local stress state.

We find a similar trend to Hopp et al. (2019, Chapter 3) when including all focal mechanisms from 2012-2015 (an increase in the number of focal mechanisms from 86 to 205) (Figure 5.6). The additional events do not change the results of the inversions in southern Ngatamariki compared to the previous study, and a normal faulting regime still prevails. The three southern clusters (4, 1 and 9) progress along strike of the Aratiatia Fault Zone from near injection well NM10 in the southwest to past injection well NM06 in the northeast. There is little variation between the three inversions for these clusters. For each, $\sigma_{1} \approx \sigma_{V}, \mathrm{~S}_{\text {Hmax }}$ is oriented $\sim 045^{\circ}$ and the stress ratio is low, $\nu=0.3-0.4$.

In the north, the stress state for Cluster 2 is unsurprisingly similar to the results presented by Hopp et al. (2019. Chapter 3), given that most of the events in this cluster are common between the two datasets. Cluster 6 includes only events that have yet to be used for stress inversion. While Clusters 2 and 6 are only 300-400 m apart, the inversions reveal distinct stress states. In Cluster $2, \sigma_{1}$ dips $\sim 30^{\circ}$ at $\sim 020^{\circ}$, while it dips $\sim 51^{\circ}$ at $\sim 090^{\circ}$ in Cluster 6 , forming a girdle with $\sigma_{2}$, indicating a stress ratio, $\nu$, approaching zero.

\section{Rotokawa}

At Rotokawa, the hypocenters for the focal mechanisms shown in Figure 5.3 reveal the same two NE-SW striking structures discussed in Chapter 4, which we continue to interpret as being the Central Field Fault (CFF) and Injection Field Fault (IFF) from west to east, respectively. As these structures are known to act as cross-strike barriers 

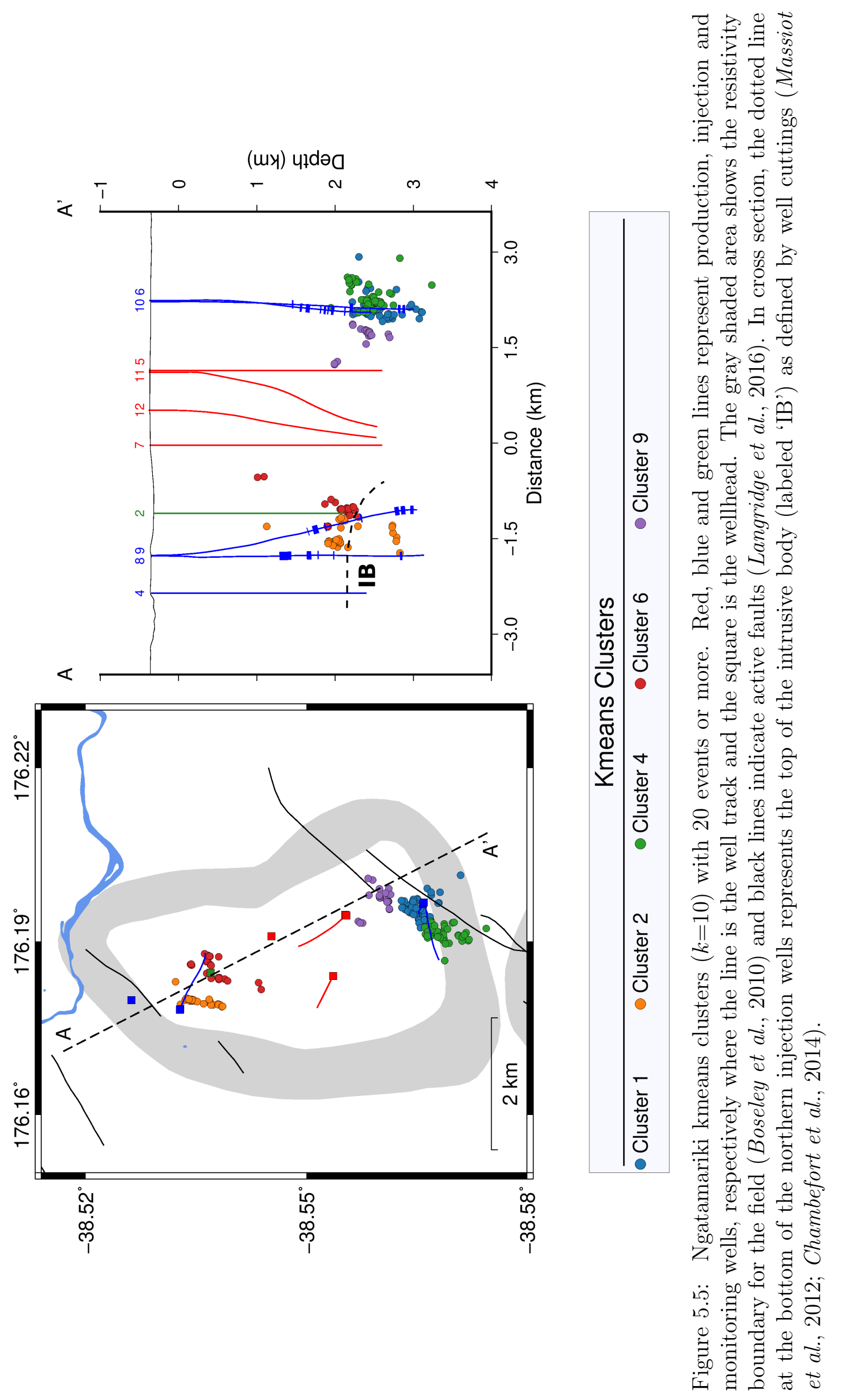


\section{Northern}
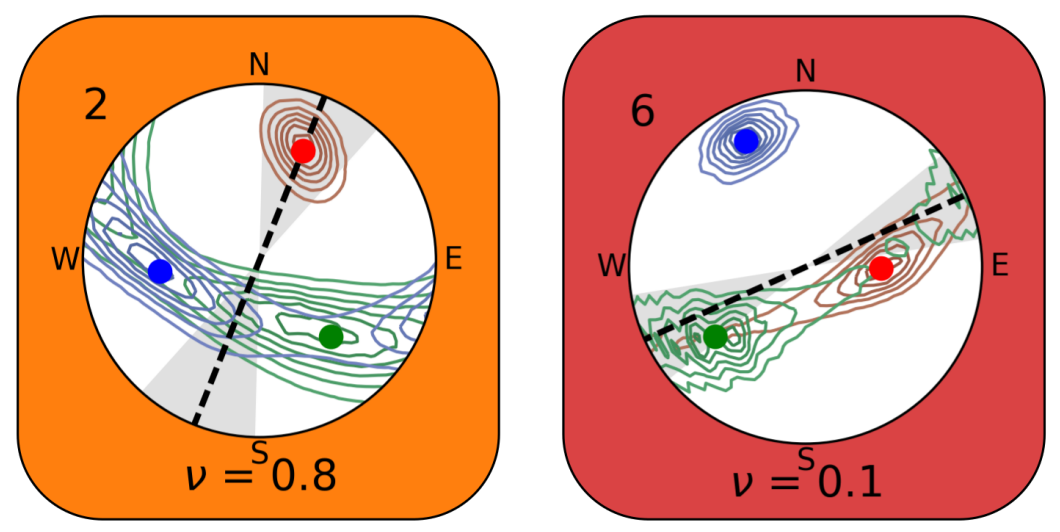

\section{Southern}
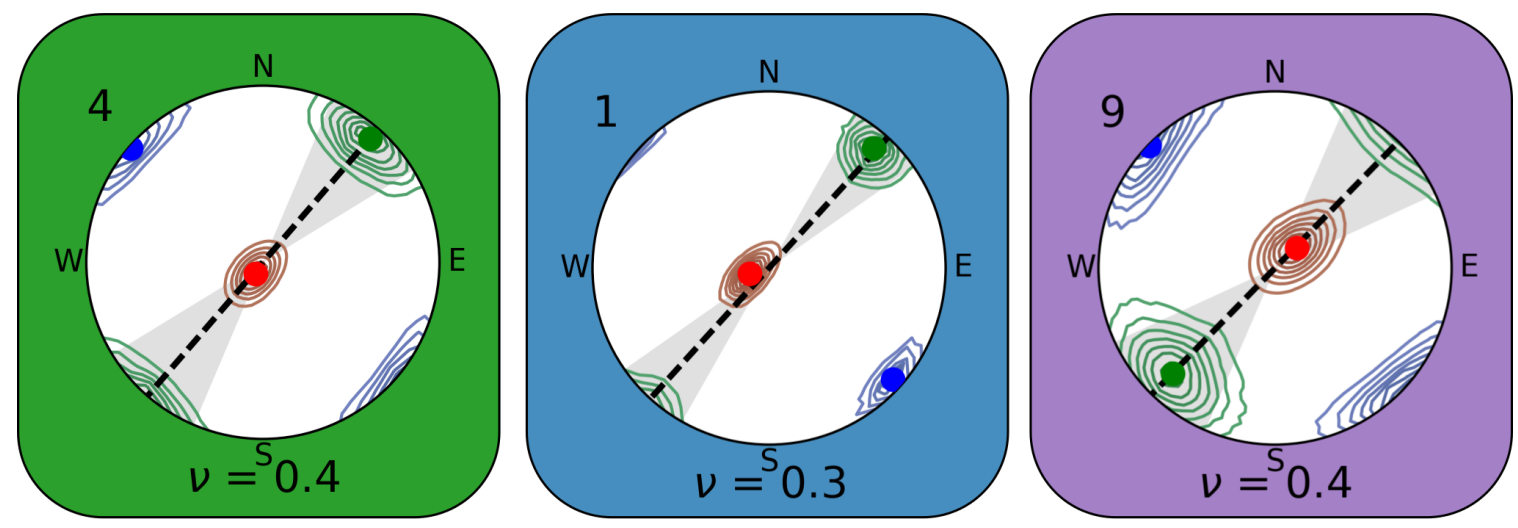

Figure 5.6: Lower hemisphere stereonets representing the stress inversion results for each kmeans cluster shown in Figure 5.5. Red corresponds to $\sigma_{1}$, green to $\sigma_{2}$ and blue to $\sigma_{3}$, where the filled circle represents the maximum likelihood axis and the contours represent the shape of the pdf for that axis. The black dotted line shows the direction of maximum horizontal compressive stress $\left(\mathrm{S}_{\text {Hmax }}\right)$ for which the gray shaded bowtie shows the $90 \%$ confidence region. Each plot is annotated below with the calculated stress ratio, $\nu$. Stereonet backgrounds are colored-coded to match the corresponding cluster in Figure 5.5.

to fluid flow, we choose to divide the reservoir into distinct 'compartments' bounded by these structures (Figure 5.7). Our chosen kmeans clustering parameter, $k=30$, produced 14 clusters, each containing a minimum of 20 focal mechanism observations. We then group these clusters into compartments as follows:

- The western compartment (light green/blue, Figures 5.7 and 5.8), contains all clusters northwest of the IFF and southeast of the CFF. These are Clusters 7, 2, 9, 12 and 17, shown in Figure 5.8

- The southeastern compartment (pink, Figures 5.7 and 5.8), contains clusters southeast of the IFF and south of the cross-strike structure inferred from the offset in focal mechanism locations along strike of the IFF. These are Clusters 0, $3,25,13,14$, and 15, shown in Figure 5.8 
- The northeastern compartment (coral, Figures 5.7 and 5.8), contains the remainder of the clusters southeast of the IFF (Clusters 10 and 23, Figure 5.8).

- The final cluster, Cluster 28, is located just inside the northern production field. We infer the centroid of this cluster to be outside the bounds of the compartments described above.

Figure 5.8 shows the stress inversion results for each cluster, divided by compartment and color coded to match Figure 5.7. From a first glance, it is clear that each inversion shows a normal faulting regime, with $\sigma_{1} \approx \sigma_{V}$. Only minor variations in the plunge of $\sigma_{1}$ are observed, but these are within the bounds of focal mechanism uncertainties $\left(\sim 30^{\circ}\right.$ angular deviation of the $\mathrm{P}$ axis $)$.

Figure 5.8 also shows stress inversions that include all focal mechanisms in each compartment (white background, labeled 'ALL'). In the western compartment, $\mathrm{S}_{\text {Hmax }}$ is oriented $056^{\circ}$ while in the northeast compartment, $\mathrm{S}_{\text {Hmax }}$ is oriented $053^{\circ}$. For both of these compartments, $\nu$ approaches 1 . In the southeast compartment, $\mathrm{S}_{\text {Hmax }}$ is oriented $021^{\circ}$ with a $\nu$ of 0.65 . As the values of $\nu$ approach 1.0 throughout most of the reservoir, the uncertainty in $\mathrm{S}_{\operatorname{Hax}}$ is high due to the ambiguity in the orientations of $\sigma_{2}$ and $\sigma_{3}$.

\subsection{Discussion}

\subsubsection{Ngatamariki}

\section{Stress inversion}

The relatively small number of focal mechanisms calculated for Ngatamariki (205) precludes the extensive clustering that would allow us to analyze temporal variations in the stress state at the reservoir, as has been done elsewhere (e.g. Martínez-Garzón et al. 2013). The five clusters shown in Figures 5.5 and 5.6 reveal spatial variations similar to those found by Hopp et al. (2019, Chapter 3). As expected, the three clusters in the southern injection field show a normal faulting regime with NE-SW $\mathrm{S}_{\text {Hmax }}$, consistent with all previous studies of stress in the region (Hurst et al. 2002 , 2008; Townend et al., 2012). The stress ratio, $\nu$, in Cluster 1 , closest to the main injection well, NM06, is lower than in Clusters 4 or 9 (0.3 as compared to 0.4), but the uncertainties overlap considerably, and we therefore will not comment on their significance.

In the northern injection zone at Ngatamariki, Clusters 2 and 6 (Figure 5.6) indicate a strongly deviated stress regime with no vertical principal stress axis. Between the two clusters, both $\nu$ and $\mathrm{S}_{H \max }$ vary considerably, as do the orientations of all three principal stress axes. $\mathrm{S}_{\text {Hmax }}$ is $022^{\circ}$ and $066^{\circ}$ for Clusters 2 and 6 , respectively, while $\nu$ is 0.8 and 0.1 . The striking contrast between these two clusters, with centroids located 


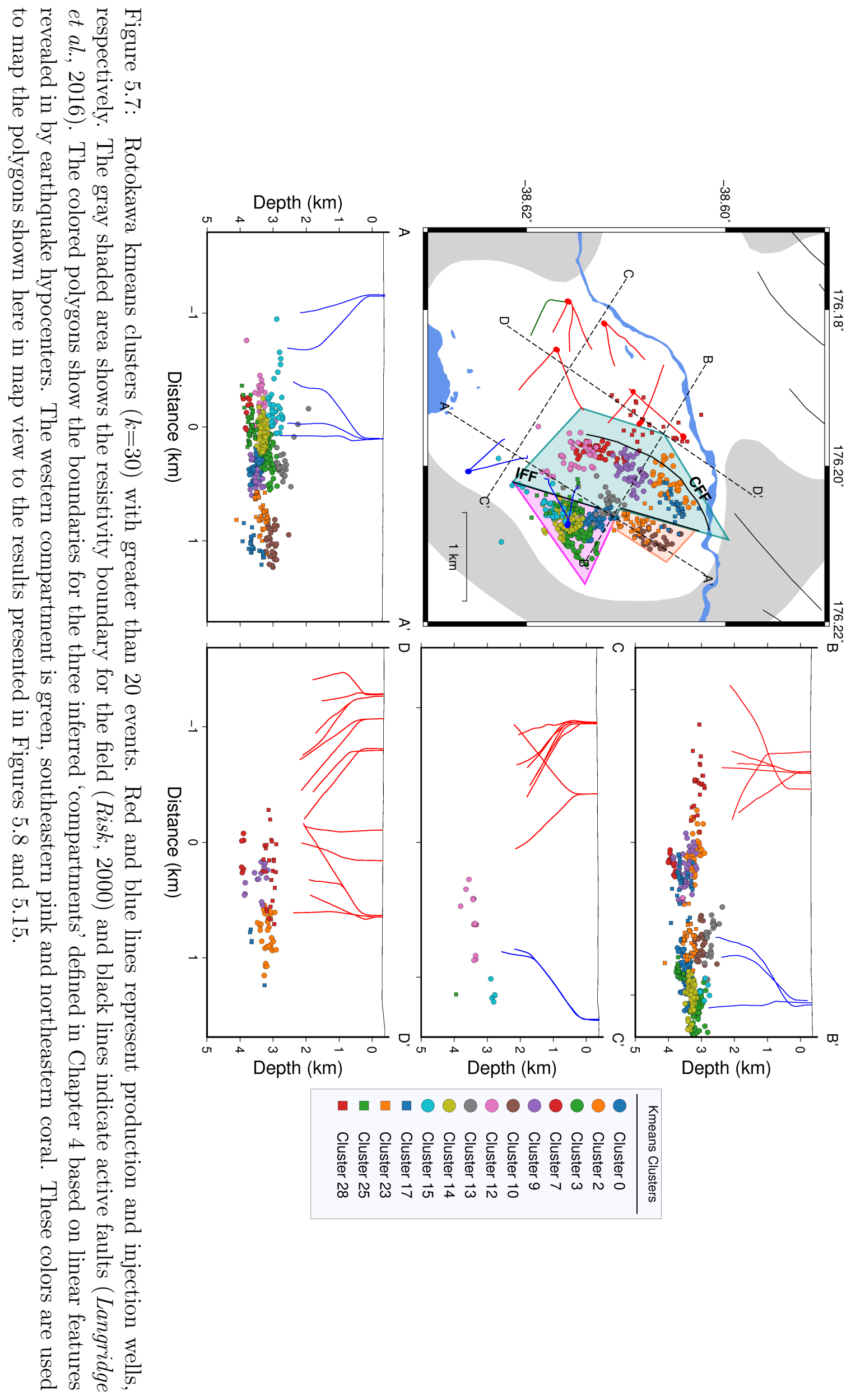



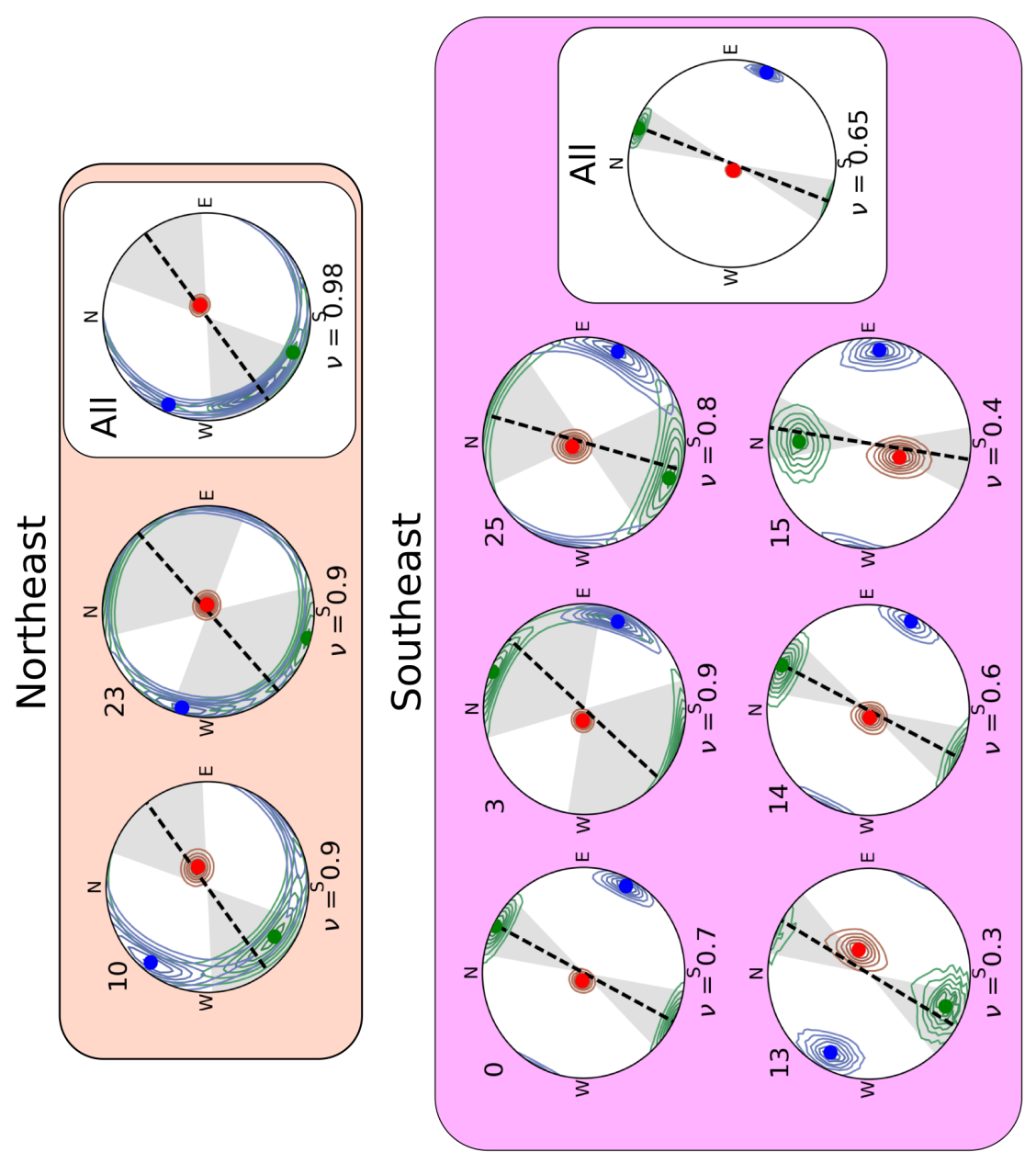

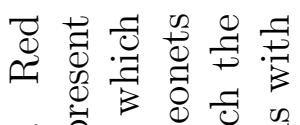

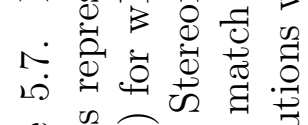

๑

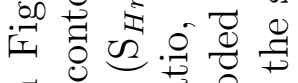

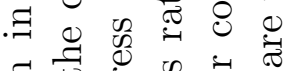
F

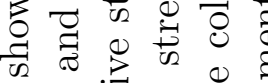

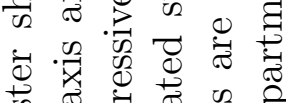
热

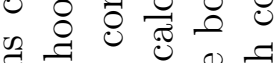

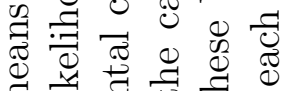

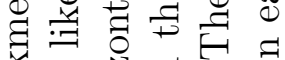
켜

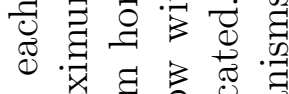

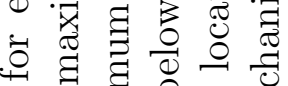

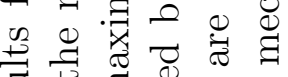

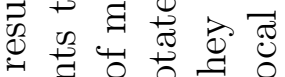

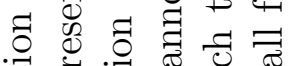
क्षे

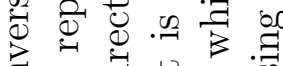

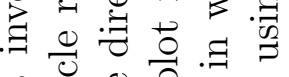

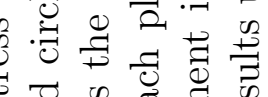

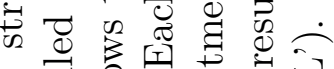

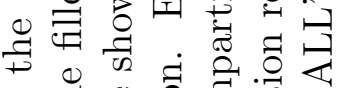

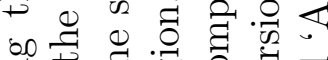
.尹口孔 : 至苛

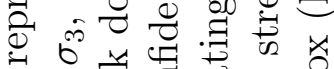

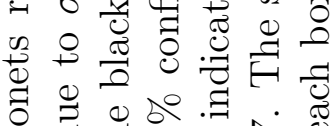
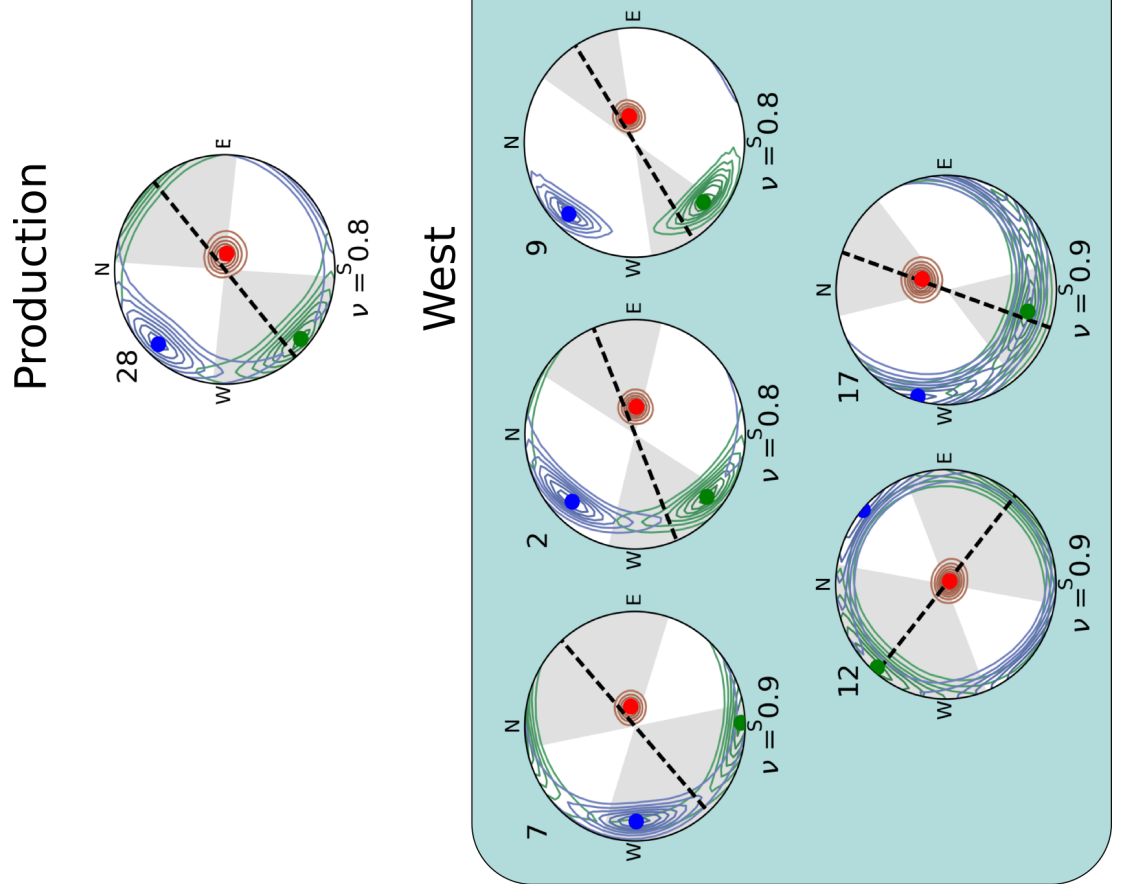

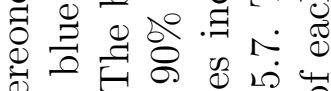
D 0 告 0 焉

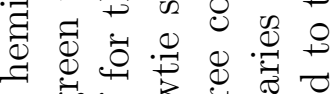
¿

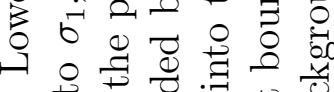

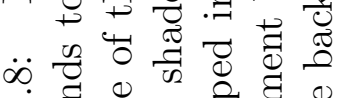
ن

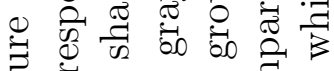

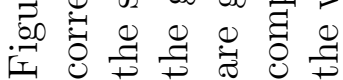


$<1 \mathrm{~km}$ apart, is puzzling. Hopp et al. (2019, Chapter 3$)$ suggest that the tonalite intrusive body, encountered in wells NM04, NM08 and NM09, may be responsible for the deviation of the northern Ngatamariki stress state from an expected normalfaulting regime. The geometry and orientation of this intrusive is poorly constrained, with cuttings found in only three wells (Chambefort et al., 2014), making it difficult to assess its effect on stress locally. Unless it is highly irregularly shaped (e.g. a series of dikes/sills) we envision the intrusive having a similar effect on the stress state at both Clusters 2 and 6, which are located at similar depths and are similar distances from the top of the intrusive. However, stress variations due to irregularities in the shape and orientation of the intrusive body (or bodies) provide the simplest explanation for the change in stress between the two clusters.

We suggest two other possible explanations for the contrast between Clusters 2 and 6 that are related to elevated pore-fluid pressure and reservoir cooling. The events belonging to Cluster 2 are likely related to injection at the nearest well, NM08, while those in Cluster 6 are located at the position of the feedzones in NM09. Figure 5.9, shows wellhead pressure and flow rate for NM08 and NM09 during the study period. As wellhead pressures are similar at each well, we do not anticipate pore-pressure perturbation having caused the discrepancy in stress state between Clusters 2 and 6 . In addition, because pore-pressure acts equally on each of the principal stress components, such large deviations in $\nu$ and $\mathrm{S}_{H \max }$ are unlikely to be pore-pressure related.

As mentioned in Section 5.1.1, thermoelastic stress reduction near injection wells can produce large deviations in the local stress state (Jeanne et al., 2015a). While the simplest explanation for the contrast in stress between Clusters 2 and 6 is related to the intrusive body, reservoir contraction due to cold water injection presents a compelling alternative explanation. The degree to which a reservoir with fracturedominated permeability contracts in response to cold water injection is related to a number of factors that describe both the reservoir (e.g. rock bulk modulus and fracture spacing) as well as the injection itself (injectate temperature and flow rate) (Stephens and Voight, 1982). Although the injectate temperature at both wells is similar, the rate of injection is not. NM08 is less permeable than NM09 and, as a result, accepts only $\sim 250 \mathrm{t} / \mathrm{h}$ as compared to the nearly $1100 \mathrm{t} / \mathrm{h}$ injected at NM09 (Figure 5.9) resulting in total injected volumes over our study period of $4.19 \mathrm{e} 6 \mathrm{~m}^{3}$ and $2.07 \mathrm{e} 7 \mathrm{~m}^{3}$, respectively. We would therefore expect the cooling-related stresses at NM09 to be more significant than at NM08.

However, the majority of events in Cluster 2 occurred during the stimulation of NM08 (duration of one month) and not over the entire study period (Figure 5.9). As these events occurred $\sim 200 \mathrm{~m}$ from the main feedzones at NM08, thermal diffusivity (measured in $\mathrm{mm}^{2} / \mathrm{s} ;$ Kanamori et al., 1968) and the short duration of stimulation make it unlikely that they were influenced by cooling from the well. In contrast, the events in Cluster 6 are well-distributed over a longer period and are therefore more likely to 

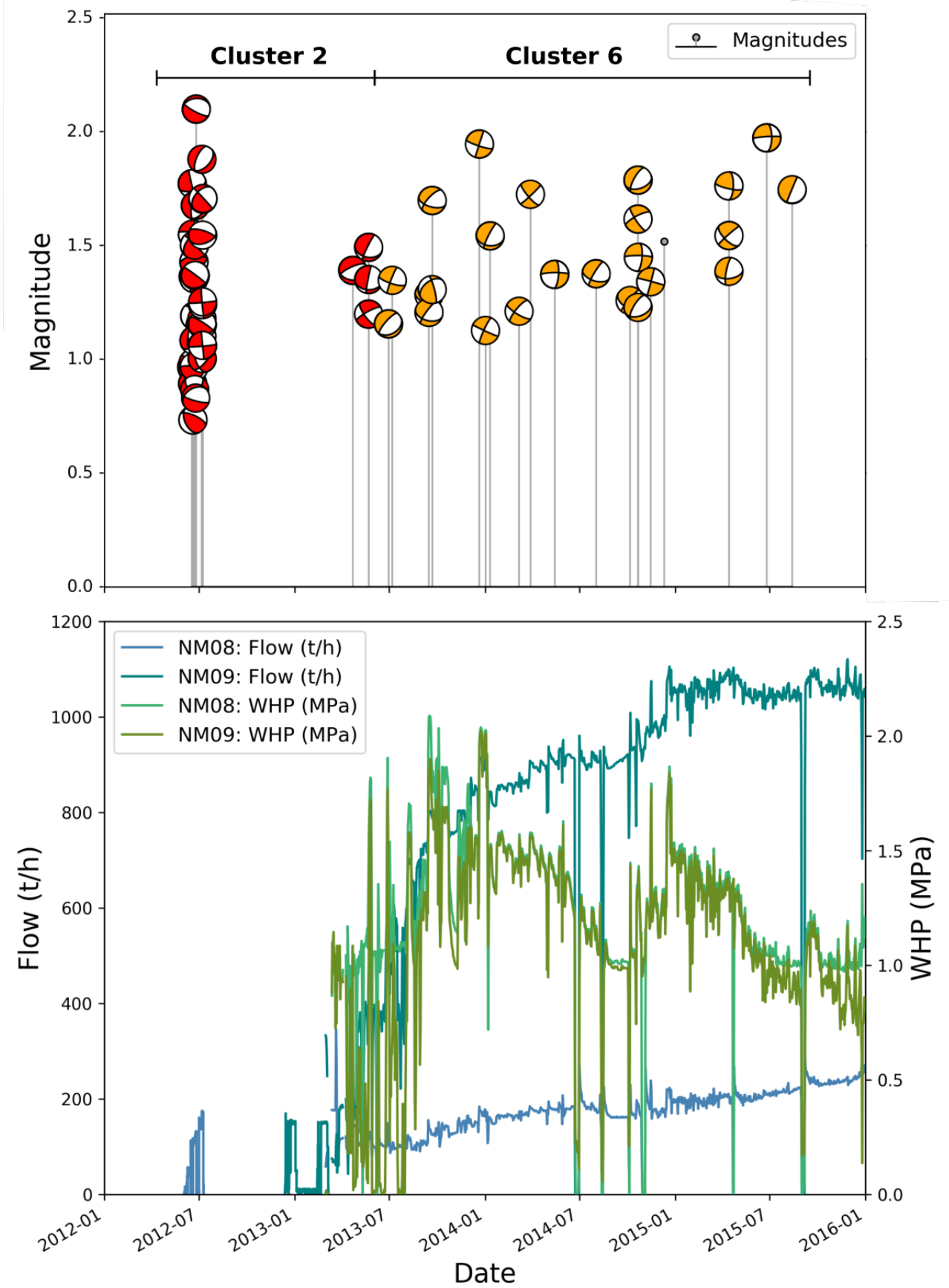

Figure 5.9: Northern Ngatamariki flow rates, wellhead pressures, and focal mechanisms. Cluster 2 (red) and Cluster 6 (orange) are shown as stems with the plan view lower-hemisphere focal mechanism solution as a head and the height corresponding to the magnitude of the event. Although the clusters were created via kmeans clustering based on Euclidian distance between events, the clusters are also perfectly split in time with Cluster 2 occurring early in the study period, and Cluster 6 occurring later.

have been influenced by thermoelastic stresses. The extent of reservoir cooling has been confirmed by pressure-temperature spinner runs at monitoring well NM02 ( 250 $\mathrm{m}$ from NM09), where temperatures at reservoir depths had cooled by $\sim 100^{\circ} \mathrm{C}$ within three years of the start of injection (Steve Sewell, personal comm.). Therefore, the 
cooling zone extending from NM09 likely encompasses most of the events in Cluster 6 and probably extends further towards the production field, although it has not yet reached well NM07 (Figure 5.5. We can be confident that the stress inversion of Cluster 6 is therefore affected by thermoelastic stresses.

If the cooled zone around NM09 is larger in the vertical direction than it is laterally (such as in the case of a vertical pipe), it will have reduced $\sigma_{1}$ by more than $\sigma_{2}$ or $\sigma_{3}$. Such a scenario may help explain the lower stress ratio and different faulting regime observed in Cluster 6 when compared with Cluster 2. A similar phenomenon was observed and modeled by Jeanne et al. (2015a) and Martínez-Garzón et al. (2013) at The Geysers, where a normal faulting regime above and below the reservoir has become a strike-slip regime within it as a result of cooling at the injection wells.

\subsubsection{Rotokawa}

\section{Spatial stress variation}

As demonstrated in Figure 5.8, deviations in the stress state at Rotokawa are less dramatic than those between northern and southern Ngatamariki. For each of the clusters at Rotokawa, $\sigma_{1} \approx \sigma_{V}$, with only minor variations in dip that cannot be confidently resolved. However, there are spatial variations in both $\mathrm{S}_{H \max }$ and $\nu$ that deserve further inspection. Specifically, values for $\mathrm{S}_{H \max }$ and $\nu$ in the southeast compartment, nearest the injection wells, are distinct to those in other parts of the field (Figure 5.8).

Borehole measurements of drilling-induced tensile failure in the production field (RK18L2, RK32, RK30L1) (McNamara et al., 2015) show that $\mathrm{S}_{\text {Hmax }}$ ranges from $025^{\circ}$ $049^{\circ}$. They also demonstrate that lateral stress changes in the reservoir are significant and can occur over distances of $<1 \mathrm{~km}$. However, as no images were obtained from any of the current injection wells, we have little constraint on the orientation of stress or fractures in the seismically-active part of the field. Inverting for the stress state using all mechanisms in the southeast compartment, $\mathrm{S}_{\text {Hmax }}$ is $021^{\circ}$ with a stress ratio, $\nu$, of 0.65. For all mechanisms in the western compartment, $\mathrm{S}_{H \max }$ is $056^{\circ}$ and $\nu$ approaches 1 , while in the northeast compartment $\mathrm{S}_{H \max }$ is $053^{\circ}$ and $\nu$ also approaches 1 . These values for $\mathrm{S}_{H \max }$ are roughly consistent with directions obtained from the borehole measurements (McNamara et al., 2015).

In Figure 5.10 we show the stress inversion results projected onto the horizontal plane as bowties showing the $90 \%$ confidence interval for $\mathrm{S}_{H \max }$, colored by the stress ratio. While it is tempting to interpret the apparent anticlockwise rotation of $\mathrm{S}_{H \max }$ in the southeast compartment (relative to the rest of the field) as related to injection well proximity, there are a number of caveats. Most importantly, stress ratios approaching 1.0 in the rest of the reservoir suggest that $\sigma_{2}$ and $\sigma_{3}$ have similar magnitudes. Given that $\sigma_{2}$ and $\sigma_{3}$ are horizontal for all clusters, stress inversion from focal mechanisms 
cannot adequately resolve the true direction of $\mathrm{S}_{\text {Hmax }}$ for a stress ratio of 1 . So while an azimuth of $021^{\circ}$ for $\mathrm{S}_{H \max }$ in the southeast compartment is relatively well constrained, interpreting it in the context of $\mathrm{S}_{\text {Hmax }}$ throughout the rest of the reservoir is unwarranted.

Although stress ratio is the least well-resolved parameter resulting from the inversion of focal mechanisms, we can at least say with some confidence that $\nu$ is lower, on average in the southeast compartment than in the rest of the reservoir. As the southeast compartment contains, or is adjacent to, injection wells RK20, RK23 and RK24, it is subject to some of the highest pore pressures and temperature gradients in the field. As discussed for Ngatamariki, it is difficult to envision a scenario in which elevated pore pressures of $\sim 1 \mathrm{MPa}$ would deviate the in situ stress state in any discernible way, especially given that pore pressure affects each principal stress axis equally. A decrease of $1 \mathrm{MPa}$ for all principal stress axes will change the stress ratio by some small amount, but this is unlikely to be measurable through focal mechanism inversion.

Instead, we infer that the only injection-related process capable of producing significant stress changes at Rotokawa is the cooling of the reservoir host rock. As injection had been occurring in the current configuration (i.e. at wells RK20-RK24) for at least five years by the end of our study period, the cooling front would have had time to reach the boundaries of our inferred southeast compartment, and likely the southern portions of the western compartment as well. Numerical modeling of The Geysers geothermal field in California has shown that stress reductions measured in 10's of MPa are possible over time spans of 2-5 years for similar injectate temperatures and in situ stress state to what is encountered at Rotokawa (Jeanne et al. $2015 \mathrm{a})$. These stress changes are greatest directly adjacent to the wells, as would be expected, because there the fluid is coldest and the induced thermal gradient is the greatest. As the cool, denser fluid enters the reservoir, it flows downward, driven by gravity. This gravity flow results in a vertically elongate zone of cooling, which preferentially reduced whichever stress axis is most vertical (Jeanne et al., 2015a). In the case of The Geysers, Rotokawa and Ngatamariki, this means preferential reduction of $\sigma_{1}$, which has the effect of limiting the population of fractures oriented for failure and reducing the stress ratio. As the injected fluid begins to heat and flow laterally, the zone of cooling also elongates in the direction of greatest lateral permeability, likely subparallel to $\mathrm{S}_{H \max }$, along strike of structures such as the IFF or CFF, which are well oriented for failure in the regional stress regime. We envision a complex interplay between the state of stress and the geometry of the zone of cooling at Rotokawa, where faults that are well-oriented for failure also define a preferred axis of cooling. It is possible that preferential reduction of $\sigma_{1}$ due to gravity-driven flow in the southeastern compartment has led to a reduced stress ratio in this portion of the field and that a limited degree of cooling further from the wells has left the stress state largely unchanged. 


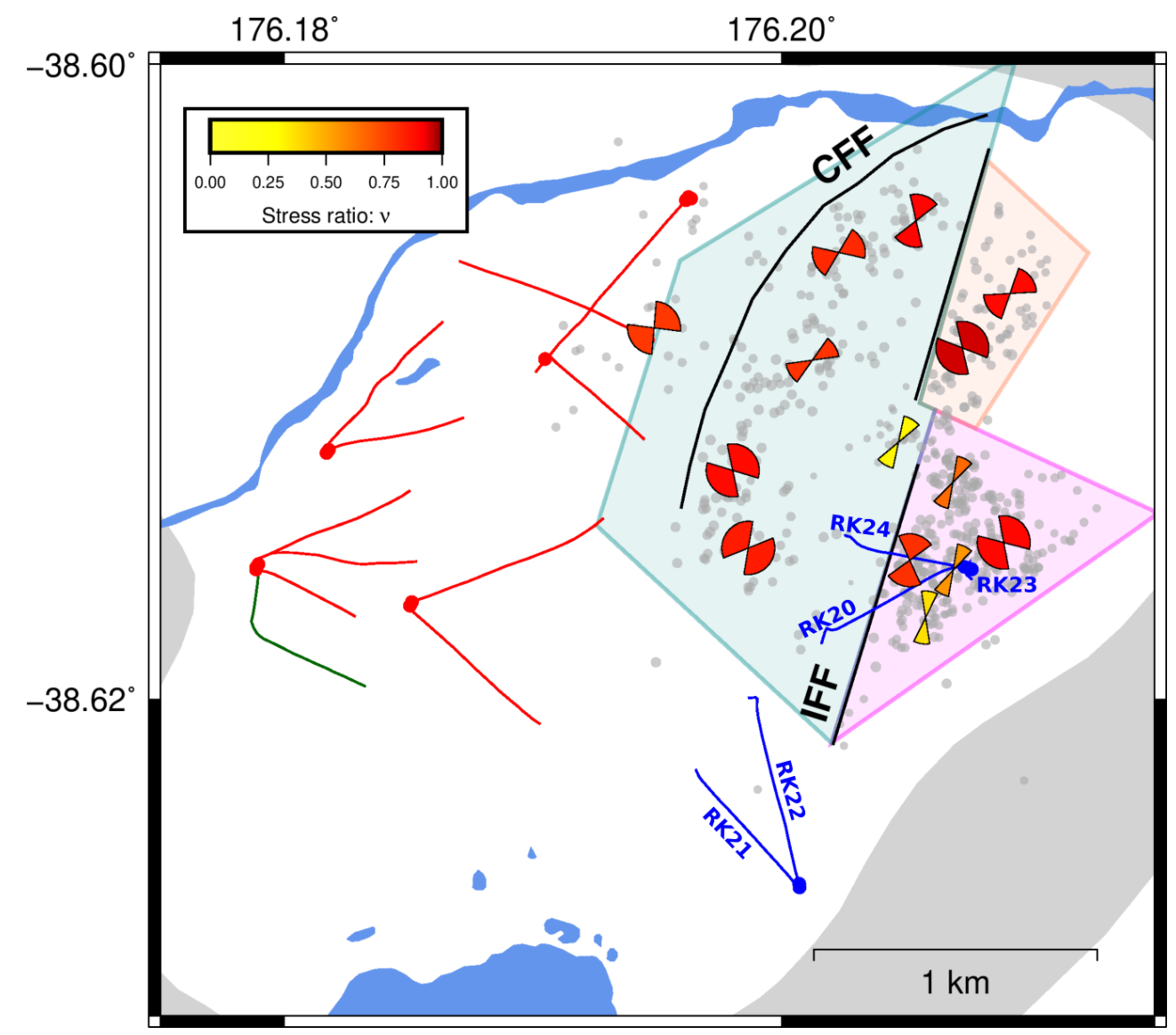

Figure 5.10: $\mathrm{S}_{\text {Hmax }}$ directions for the kmeans clusters (Figure 5.7) at Rotokawa. Bowties show the $90 \%$ confidence intervals for $\mathrm{S}_{H \max }$, colored by the stress ratio, $\nu$.

\section{Temporal stress variation}

Finally, we compare stress parameters from focal mechanism inversion to temporal changes in injection at Rotokawa. Due to the vertical $\sigma_{1}$ and high stress ratio in the western and northeastern compartments, $\mathrm{S}_{\text {Hmax }}$ is poorly constrained and we therefore avoid further subdividing these areas for temporal analysis. In the southeast compartment, the calculated stress ratio is lower than elsewhere in the field and $\mathrm{S}_{\text {Hmax }}$ is better constrained. Its proximity to injection wells means that this compartment is also the most likely to show signs of injection-induced stress change. We therefore divide the 304 focal mechanisms in this compartment into time-sequential groups of 55 events, and invert for the stress states in each group, advancing the inversion by steps of 10 events between data points. As $\sigma_{1} \approx \sigma_{V}$ for all clusters at Rotokawa, we plot $\mathrm{S}_{\text {Hmax }}$ and $\nu$ with time versus the injection rates from RK20, 23 and 24 (Figure 5.11).

Both $\mathrm{S}_{\text {Hmax }}$ and $\nu$ appear to respond to the injection changes at wells RK23-24 during the last half of 2013. $\nu$, in particular, decreases from near 1.0 to below 0.2 over the six months following the injection shift from RK24 to RK23. If our interpretation of the orientation of the Injection Field Fault is correct, and it also serves as a barrier to cross strike flow, then the stress state to the east of the IFF would respond most 

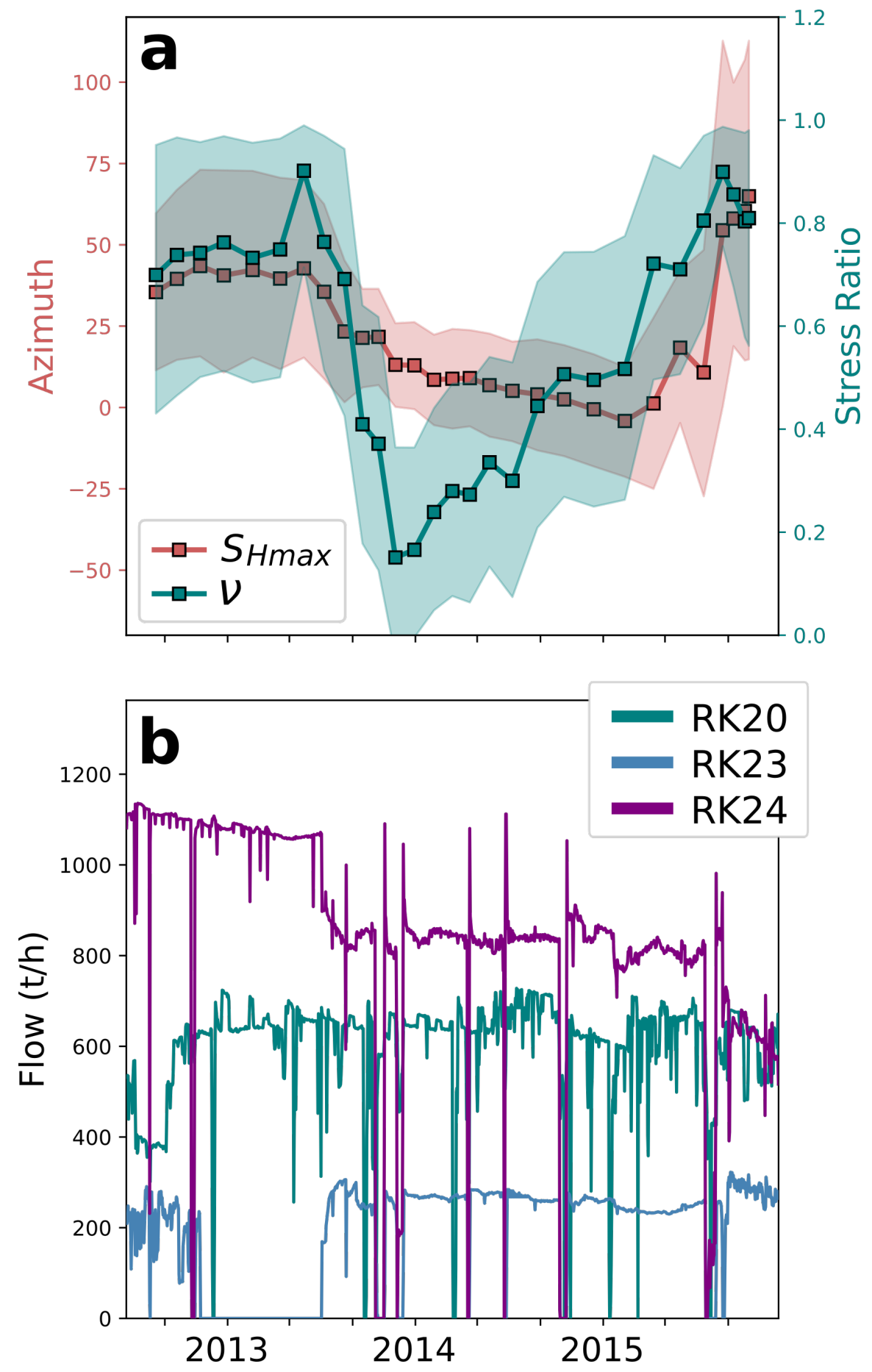

Figure 5.11: a) Stress parameters $\nu$ (green) and SHmax (red) in the southeastern compartment at Rotokawa with time. Each data point corresponds to a cluster of 55 consecutive focal mechanisms with an overlap of 45 events between adjacent data points. Points are plotted at the time average for each subset of events and the shaded regions represent the 90 percent credible intervals for each time step. b) flow rates for injection wells RK20, RK23 and RK24 during the study period.

strongly to RK23, which is the only well on the eastern side of the fault. If this is the case, then the decrease in $\nu$ and anticlockwise rotation of $\mathrm{S}_{H \max }$ shown in Figure 5.11 may correspond to the restart in injection at RK23 after $\sim 8$ months of inactivity. 
As described in Section 5.4.1, such a decrease in $\nu$ could reflect preferential cooling of $\sigma_{1}$ (Figure 5.12 Equation 5.1). This makes sense in the context of a well that has been inactive for a number of months. During this time, the near-well reservoir would have had ample time to re-heat to near the natural state temperature. Once injection restarted (Figure 5.12 ), the temperature contrast would have encouraged gravitydriven, downward flow from the well and a corresponding decrease in $\nu$. Interestingly, $\sim 7$ months after the restart of injection at RK23, $\nu$ began to rebound, eventually returning to elevated levels $(>0.8)$ by the end of the study period. The steep drop and more gradual rebound of $\nu$ may tell us something about the time scale and geometry of reservoir cooling. If a decrease in $\sigma_{1}$ corresponds to the drop in $\nu$ due to downward flow, perhaps the rebound in $\nu$ reflects a transition from downward to lateral flow (Figure 5.12 b), likely along fractures subparallel to $\sigma_{2}\left(\mathrm{~S}_{H \max }\right)$. As flow transitions from gravity-driven to lateral, the shape of the cooled zone of the reservoir changes from a sub-vertical cone beneath the well to one with a larger lateral footprint. If lateral permeability is anisotropic, favoring flow in the direction of $\mathrm{S}_{\text {Hmax }}$, as we would expect, then the zone of cooling would also be elongate in this direction, thereby cooling

$\mathrm{S}_{H \max }$ more than $\sigma_{3}$ (Figure $5.12 \mathrm{~b}$ ). Such a scenario could produce an increase in $\nu$ similar to what we observe in the southeast compartment at Rotokawa.

If our interpretation is incorrect, and RK23-24 inject into the same pressure compartment, then it may be difficult to distinguish the contribution of each well to variations in stress. In this case, RK20 and RK24 may also contribute to the temperature and pressure variations in the southeast compartment and the observed decrease in $\nu$ may also be related to the decrease in flow at RK24.

\subsection{Conclusions}

In this work we present 982 focal mechanisms calculated from P-wave first motion polarities for the Ngatamariki (205) and Rotokawa (777) geothermal fields in the central Taupō Volcanic Zone of New Zealand. Mechanisms at both fields are predominantly normal and strike-slip, with small or insignificant reverse components (Figures 5.2, 5.3 and 5.4). We have divided this catalog into various spatial and temporal clusters and inverted each for the stress tensor, seeking to determine how stress varies across the fields and to assess possible relations to fluid injection activities.

Using a kmeans spatial clustering approach, we creat five clusters at Ngatamariki and 14 at Rotokawa, each with a sufficient number of focal mechanisms to reliably determine the stress state from the cluster. At Ngatamariki, we show that the stress state is distinct between the northern and southern injection zones, with a normal faulting regime in the south $\left(\mathrm{S}_{H \max } \approx \mathrm{NE}-\mathrm{SW}\right)$ and an oblique normal faulting regime in the north with no vertical principal stresses. There are too few events at Ngatamariki 


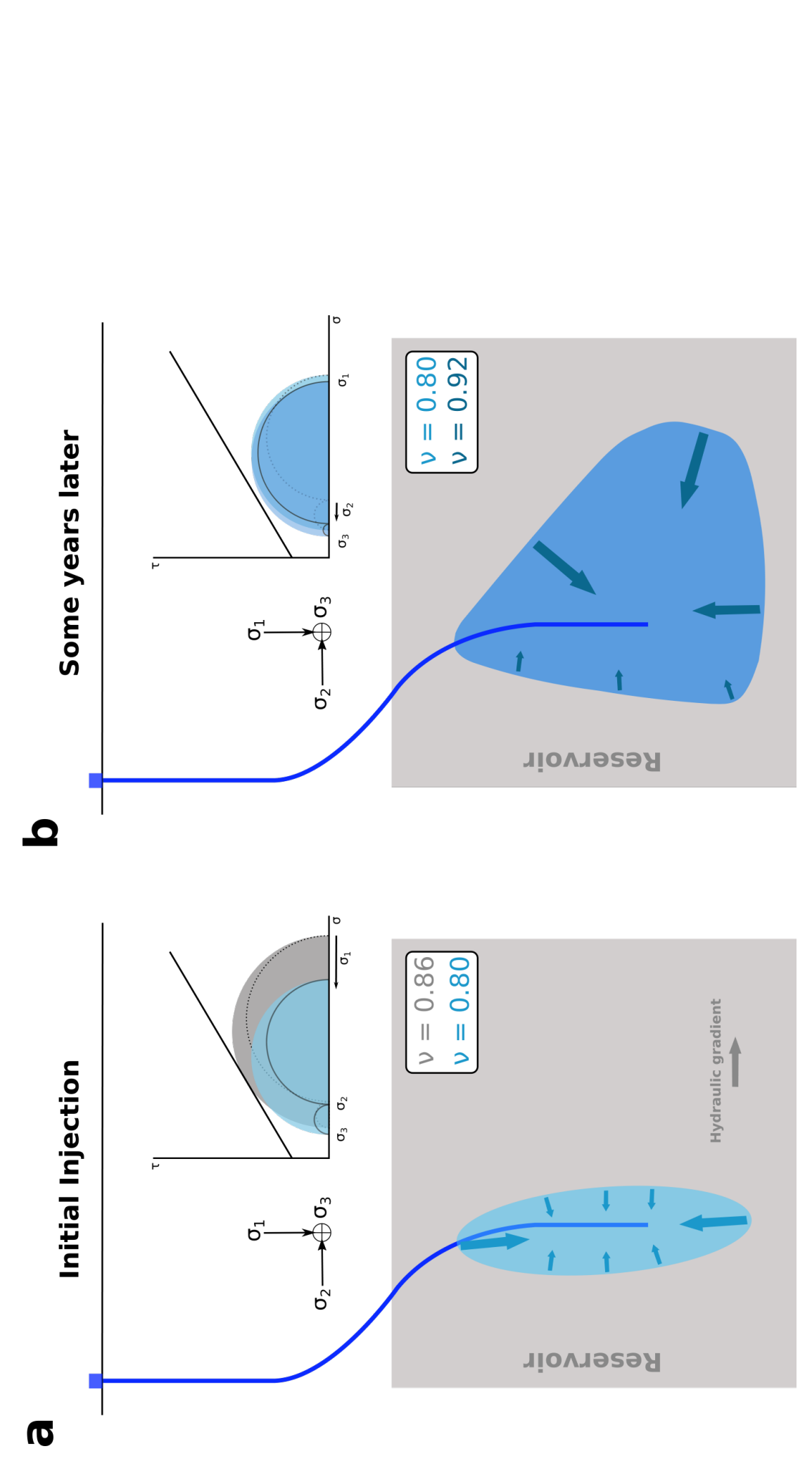

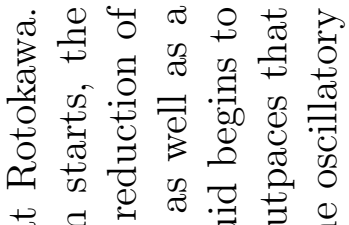

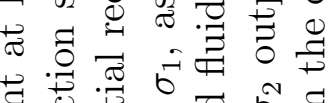

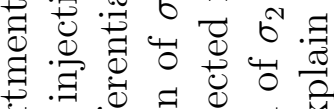

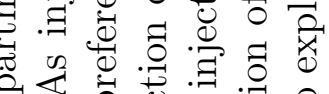

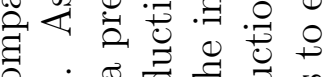

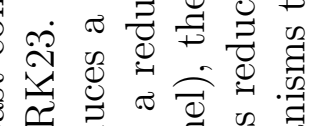

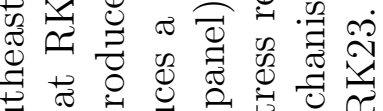

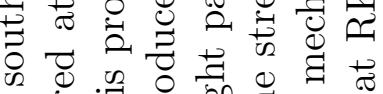

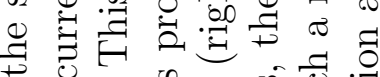

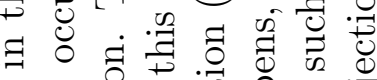

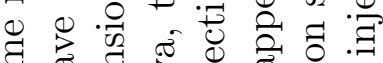

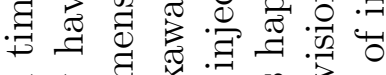

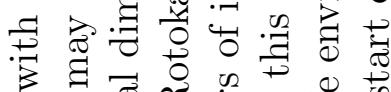

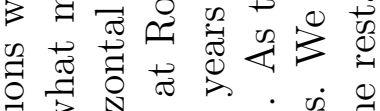

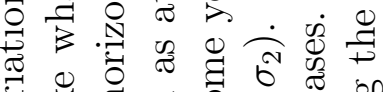

范

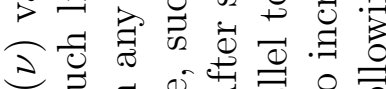

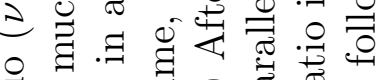

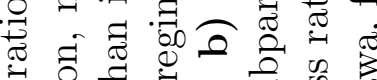

$\overrightarrow{0}$.

密. 语.

क्ष

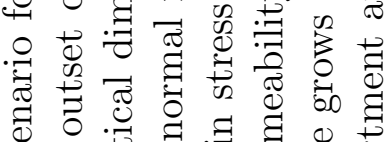

诰

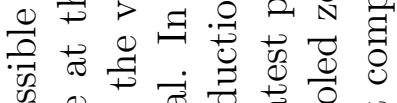

足.

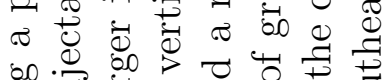

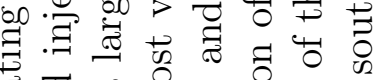

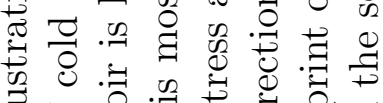

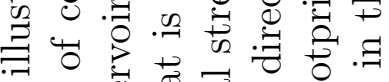

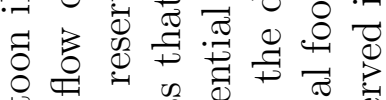

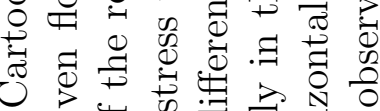

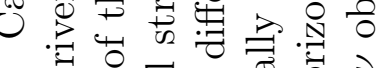

ن

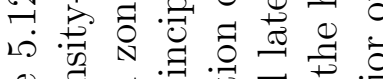

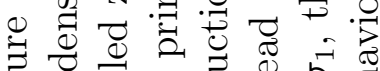

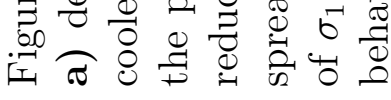


to divide the catalog into meaningful temporal clusters and it is therefore difficult to comment on the effects of fluid injection on the stress state. However, we suggest that the spatial variation in the stress inversions for Clusters 2 and 6 in the northern injection zone is related to reservoir cooling processes.

At Rotokawa, the large number of focal mechanisms (777) allows for better spatial and temporal resolution from our stress inversions. Although the stress results show exclusively normal faulting $\left(\sigma_{1} \approx \sigma_{V}\right)$ for all clusters, spatio-temporal variations in the direction of $\mathrm{S}_{H \max }$ and stress ratio, $\nu$, suggest that injection operations may be affecting the stress state. In particular, temporal variations in stress state, specifically $\nu$, for a subset of events nearest the injection wells indicate that this parameter may be sensitive to variations in injection flow rate. We suggest that this may reflect changes in the size and geometry of the cooled zone of the reservoir around the wells.

The findings presented here constitute the first study of the stress variation in the Ngatamariki field and the first attempt to characterize stress from focal mechanisms at Rotokawa. The results confirm the expected first-order normal faulting regime with NE-SW $\mathrm{S}_{\text {Hmax }}$ at both fields but show significant variation in space, especially at northern Ngatamariki where the stress field varies significantly from the south. From an operational point of view, the knowledge of the stress state away from logged wells aids in identifying the population of fractures that are well-oriented for failure, and therefore the most likely to host fluid flow. This knowledge is critical for planning injection and production strategies and for targeting wells.

\subsection{Appendices}

\subsubsection{Focal mechanism and stress inversion parameter sensi- tivity}

Figure 5.13 a shows the focal mechanism uncertainties for the entire time period of our study, with $\kappa$ as the concentration parameter of the Matrix Fischer distribution of the focal mechanisms' strike/dip/rake. Arnold and Townend (2007) established the following relationship between $\kappa$ and the standard deviation of the focal mechanism in degrees (the angular deviation is the same for each of the strike, dip and rake)

$$
\sigma_{\Theta}=\exp (3.9155-0.5659 \log \kappa)
$$

Even with numerous changes to the network in 2013 (Figure 2.2), the average uncertainties in strike, dip and rake are between 30 and 34 degrees throughout. Figure $5.13 \mathrm{~b}$, a sanity check, shows the expected negative relationship between the number of polarity recordings and the uncertainty of the resulting focal mechanism. 


\section{Focal mechanism uncertainty}
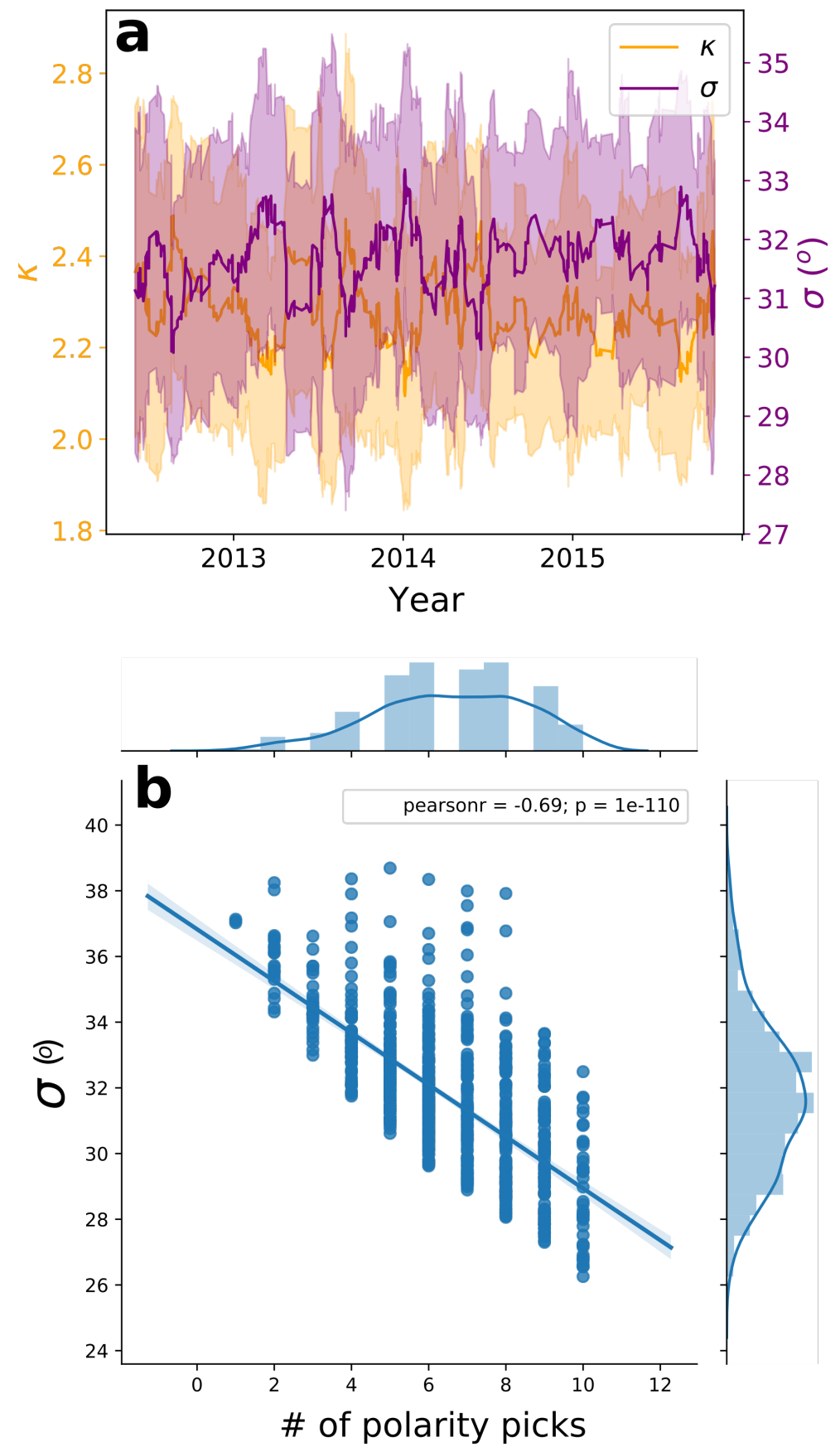

Figure 5.13: a) Concentration parameter, $\kappa$ and associated scalar standard deviation, $\sigma$, for all focal mechanisms at Rotokawa. The plot shows the mean (line) and standard deviation (1- $\sigma$, shaded area) of a moving window of 20 focal mechanism observations. b) Sanity check on the angular deviation versus the number of polarity observations for each focal mechanism. As expected, more polarity observations better constrain the resulting focal mechanism. The line is the result of a linear regression with the $r^{2}$ and $\mathrm{p}$ values noted in text.

Figure 5.14 shows alternate results for the temporal stress inversion shown in Figure 5.11 with differing windowing parameters. Whereas the clusters in Figure 5.11 contain 
55 events with a 45 event overlap, Figure 5.14 a shows clusters of 30 events with a 20 event overlap and Figure 5.14 c shows clusters of 25 events with a 15 event overlap. The purpose of these plots is to show that the drop in stress ratio, $\nu$, is robust to the smoothing and windowing parameters in the temporal clustering.
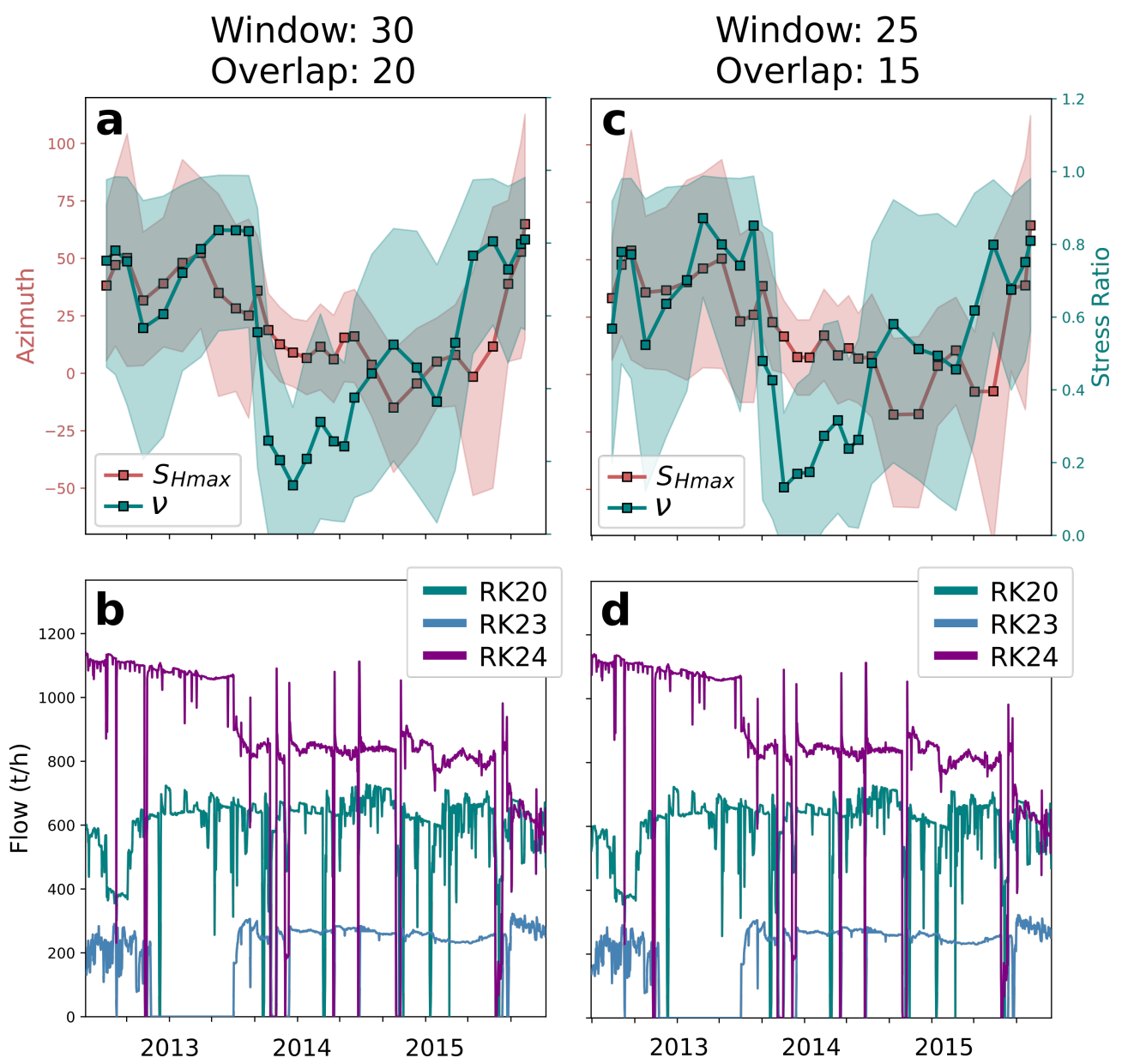

Figure 5.14: a-c) Stress parameters $\nu$ (green) and SHmax (red) in the southeastern compartment at Rotokawa with time. Each data point corresponds to a cluster of 30 (a) or 20 (c) consecutive focal mechanisms with an overlap of 20 (a) or 15 (c) events between adjacent data points. Points are plotted at the time average for each subset of events and the shaded regions represent the 90 percent credible intervals for each time step. b-d) flow rates for injection wells RK20, RK23 and RK24 during the study period.

\subsubsection{AFIT and FMI logs}

Borehole image logs use either the variations in electrical resistivity (Formation Micro Imager: FMI) or amplitude variations in acoustic reflections off the borehole wall (AFIT: Acoustic Formation Imaging Technology) to construct an image of the well with a resolution of $<5-10 \mathrm{~mm}$ (Massiot et al. 2015a; McNamara et al., 2015). Structures intersecting the wellbore appear as sinusoids in the unwrapped image, from which 
the orientation of individual fractures, bedding planes and geologic contacts can be determined (Massiot et al., 2015a). For the two available FMI logs (wells NM09 and NM10), we report only 'non-halo', conductive fractures as interpreted by Halwa (2013a) and Halwa (2013b), which have the highest likelihood of being open and not filled with a sealing mineral such as pyrite or calcite. These are the strongest candidate fractures for hosting fluid flow in the reservoir, and accommodating seismic slip. The remaining logs referenced in this work were collected via AFIT, and are therefore unable to distinguish between sealed and unsealed fractures (Massiot et al., 2012, 2015a). For AFIT logs, we report all fractures of both high and low confidence identified in the well log reports Massiot et al., 2012; Massiot and McNamara, 2010, 2011; McNamara, 2011, 2010).

At Ngatamariki, logs were collected at wells NM08 and NM09, in the northern injection zone, and well NM10 in the southern injection zone Massiot et al., 2012; Halwa, 2013a, b). While the dominant strike across all wells in the field is NE-SW, dips differ between wells. Fractures in NM08 strike $\sim 030^{\circ}$ and dip to the SE at an average of $\sim 65^{\circ}$, while those in NM09, $\sim 200 \mathrm{~m}$ to the southeast, strike $\sim 211^{\circ}$ and dip NW at $\sim 60^{\circ}$. This likely indicates the presence of antithetic structures, one intersecting NM08, dipping southeast, and the other intersecting NM09 dipping northwest (Massiot et al. 2012; Halwa, 2013a). Fractures at NM10 strike an average of $\sim 008^{\circ}$ and dip $\sim 62^{\circ}$ with more dipping to the southeast than northwest. There are also two subordinate strikes at NM10, one at $\sim 360^{\circ}$ and another at $\sim 260^{\circ}$. The bi-modal dip distribution and complex distribution of strikes likely reflect the complex structure within the Aratiatia Fault Zone, into which NM10 is drilled (Figure 5.1).

At Rotokawa, borehole images were collected for four wells in what is now the production field: RK18L2, RK32, RK30L1 and RK29 Massiot and McNamara, 2010, 2011; McNamara, 2011, 2010). As at Ngatamariki, the majority of fractures strike NE-SW but dip in accordance with the nearest large structure (i.e. PFF, CFF, IFF. Dips at well RK18L2, RK30L1 and RK29 are to the northwest at $\sim 69^{\circ}, \sim 71^{\circ}$ and $\sim 68^{\circ}$, striking $\sim 199^{\circ}, \sim 223^{\circ}$ and $\sim 161^{\circ}$, respectively. Fractures at RK32, nearest the Production Field Fault, strike an average of $\sim 052^{\circ}$ with a dip of $\sim 70^{\circ} \mathrm{SW}$.

\subsubsection{Borehole vs focal mechanism fracture orientation}

To accompany the stress work detailed in the main body of this chapter, we have also used focal mechanism solutions to characterize the orientation of the fracture network within the area of active seismicity. This is of critical importance to geothermal operators as the orientation of open fractures reveals aspects of the flow pathways from injection to production wells. Improved understanding of these pathways allows reservoir engineers to better plan injection and production strategies in order to minimize cooling and pressure drawdown. 
While focal mechanism solutions are described equally well by two orthogonal nodal planes, we can calculate which is the most likely to fail for a given stress regime. Failure increases fracture permeability through misalignment of asperities on opposing fracture faces (a process known as self-propping). Therefore, by calculating the fractures which are best oriented for failure in a given stress regime, we are also determining the fractures which are most likely to provide pathways through which reservoir fluid will flow (Townend and Zoback, 2000).

Figures 5.15 and 5.16 compare the orientations of fractures measured from borehole imaging with those inferred from the most likely failure planes of the focal mechanisms analyzed above. In order to determine which plane was the most likely to fail, we used the approach of Vavryčuk (2014), who define the instability of a plane as:

$$
I=\frac{\tau-\mu(\sigma-1)}{\mu+\sqrt{1+\mu^{2}}}
$$

where $\tau$ and $\sigma$ are the shear and normal tractions on a given plane, respectively and $\mu$ is the coefficient of static friction. We invert all focal mechanisms for the stress tensor at each field separately. We then use the whole-field stress result to calculate the least stable planes for each focal mechanism in the corresponding field.

In Figures 5.15 and 5.16, we have divided both the borehole imaging results and focal mechanisms into the major spatial clusters for comparison. In Ngatamariki we divided the data into northern and southern injection wells and the corresponding focal mechanism clusters (Figure 5.15). The image logs for northern wells NM08 and NM09 have highly uniform fracture orientations striking NE-SW, dipping SE at NM08 and NW at NM09. The most likely nodal planes also show a preference for NE-SW strike in this portion of the reservoir, albeit mostly dipping W-NW. In northern Ngatamariki there is also a subordinate, SSE-striking, WSW-dipping population of nodal planes that do not appear in the wellbore image logs. The reasons for this discrepancy are unclear, although there is a large degree of scatter in the fracture populations measured at the wells, which means that SSE-striking fractures do exist in the wells, albeit in relatively limited numbers.

At Rotokawa, the image logs were collected only at wells in the production field, which therefore do not correspond directly to the area of active seismicity. We divided the focal mechanisms into the western (green), southeastern (pink) and northeastern (coral) compartments discussed in the body of the chapter above and color-coded the rose plots based on the convention established in Figures 5.7, 5.8 and 5.10. For reference, the western compartment is the compartment closest to the wells for which image logs were collected (specifically RK29 and RK30L1). With that context, the image log results at RK29 and RK30L1 compare favorably with the most-likely nodal planes in the western compartment, which exhibit SSW strikes and WNW dips with a small subordinate population of E striking, S dipping fractures and nodal planes. Although 


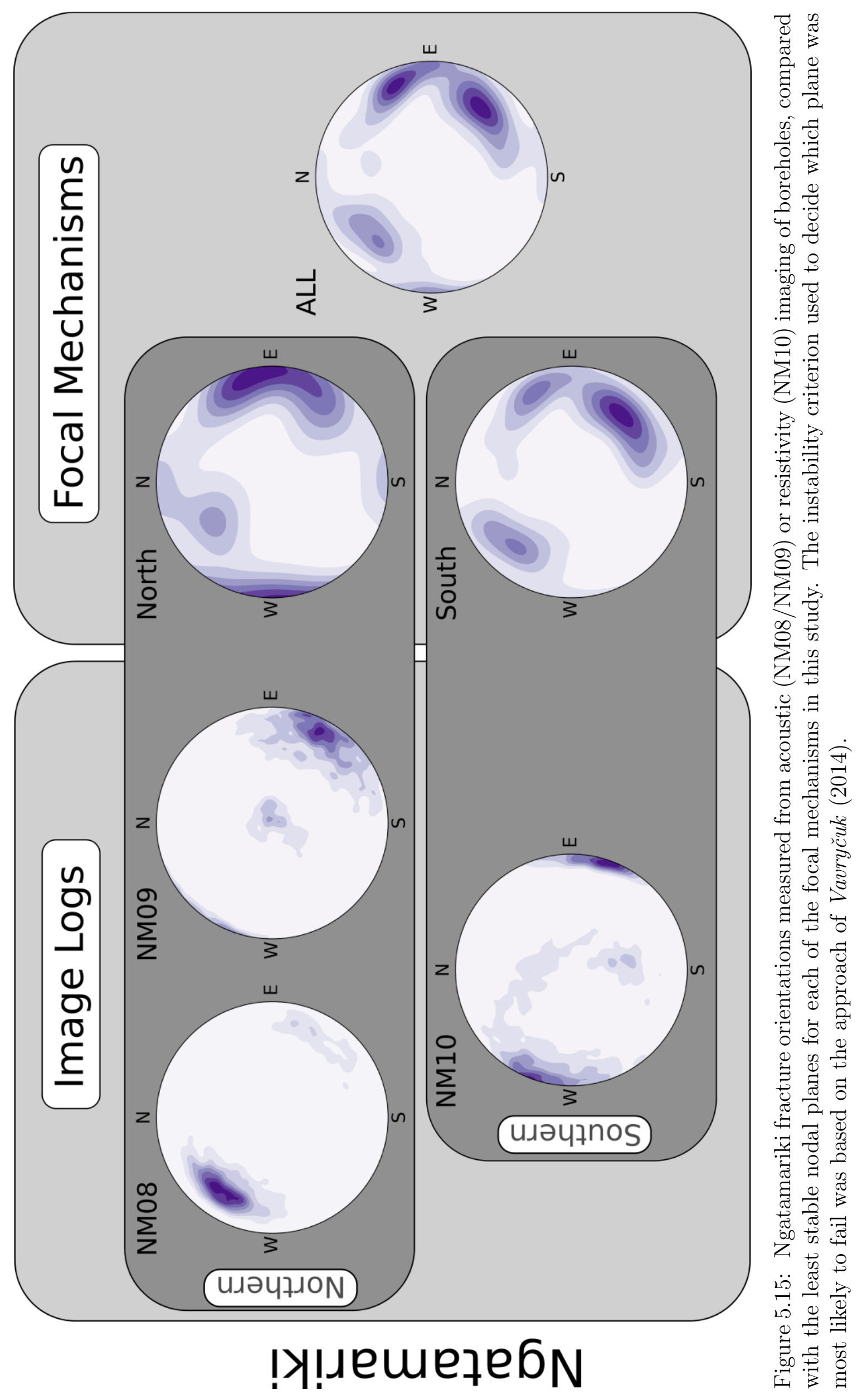




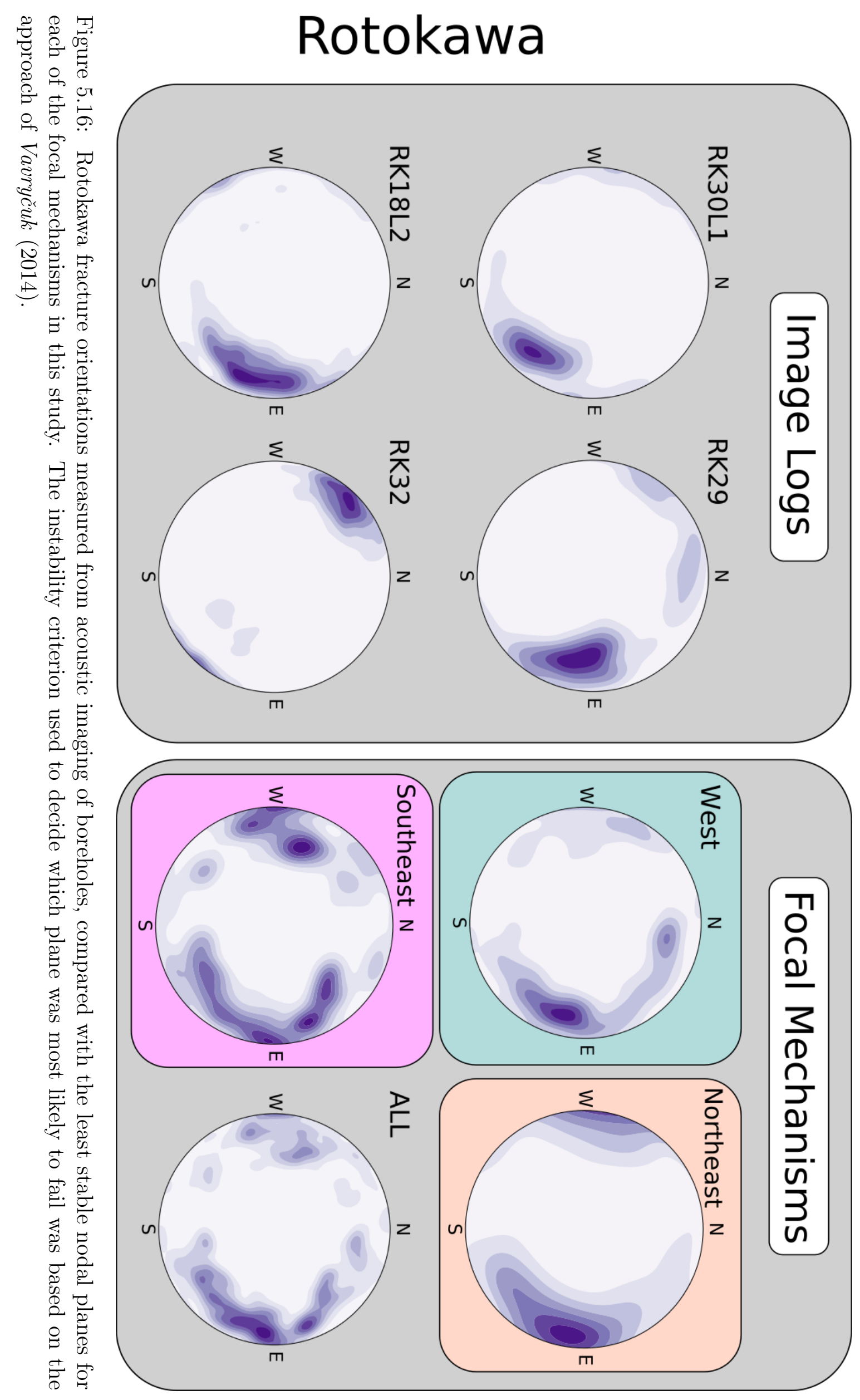


it contains fewer focal mechanisms and is located further from the logged wells, the northeast compartment exhibits a similar nodal plane distribution to the western compartment. In contrast, the southeast compartment shows a much wider distribution of likely nodal plane orientations with a dominant orientation of NNE-striking, ESEdipping fractures. We interpret this well-distributed population of unstable fractures as an indication of elevated pore-pressures in this injection-adjacent compartment of the reservoir. Elevated pore pressures should have the effect of broadening the distribution of fractures suitable for failure in a given stress regime, which has been suggested for injection operations elsewhere (e.g. Bachmann et al., 2012). This approach to fracture network characterization can be applied to sets of focal mechanisms at geothermal reservoir elsewhere and paired with the stress determination detailed above to better understand the orientation of flowing fractures and permeability away from wells. 


\section{Synthesis}

The overarching goal of this thesis is to develop a combined earthquake catalog for the Ngatamariki and Rotokawa geothermal fields in the Taupō Volcanic Zone of New Zealand, and to use it as a tool for reservoir characterization. This catalog of industriallyinduced seismicity is a manifestation of temperature and pressure change within the earth and therefore represents an opportunity to study these properties within a geothermal resource, where few direct measurements can be made. The work contained herein comprises detailed analyses of seismicity beyond the day-to-day monitoring conducted by the geothermal operators, specifically focusing on areas and time periods of intense fluid injection operations. It is our specific intent that the results presented in this work be of practical use to Mercury NZ Ltd, from the standpoint of reservoir management and planning at these fields. Our results also help illuminate the relationship between pore pressure, permeability enhancement and thermoelastic contraction, and therefore have broader implications for the understanding of seismogenesis.

\subsection{Summary}

The earthquake catalog presented in this thesis was constructed from a single network of continuously-recording seismographs. However, as this project concerns two separate geothermal fields with distinct development histories, our analyses have been partitioned into two parts, one for Ngatamariki (Chapter 3; submitted to G-Cubed) and one for Rotokawa (Chapter 4) with a final, combined portion discussing focal mechanisms and stress state at both fields (Chapter 5). Chapter 3 presents the Ngatamariki earthquake catalog and analyzes its characteristics in relation to injection well drilling and stimulation prior to plant startup. Chapter 4 presents the Rotokawa earthquake catalog and discusses spatio-temporal trends in rate, location and frequency-magnitude distribution revealed therein. Chapter 5 addresses the focal mechanisms and stress state at both fields.

The specific findings of each chapter are summarized below, followed by a synthesis of their implications for each field:

Chapter 3: 
- We applied a matched-filter detection algorithm to continuous seismic data at the Ngatamariki geothermal field to increase the total number of events in our catalog, thereby allowing for a more detailed analysis than previous studies;

- Seismicity at Ngatamariki from 2012-2015 occurs in two clusters, spatially coincident with the northern and southern injection zones. Little seismicity occurs in the production field;

- Drilling, testing and stimulation treatments at wells NM08, NM09 and NM10 produce dramatically different responses in seismicity;

- The rate of seismicity and injectivity index are poorly correlated during injection well stimulation at NM08 and NM09 where injectivity exhibits logarithmic growth in the absence of seismicity;

- Stress inversions from earthquake focal mechanisms reveal distinct stress states in the northern and southern injection zone.

Chapter 4:

- Seismicity at Rotokawa from 2012-2015 is confined to the injection field, bounded to the northwest by the Central Field Fault (CFF). This pattern is broadly consistent with previous studies of seismicity in the field (Sherburn et al. 2015 , Sewell et al., 2015b);

- Our catalog reveals, for the first time, two distinct, sub-linear features within the seismically-active portion of the field. From west to east, we infer these features to be the Central Field Fault and Injection Field Fault (IFF);

- Mapping of earthquake frequency-magnitude distribution (i.e. $b$-value) shows a higher $b$-value (1.18) near injection wells than further away $(<1.0)$, suggesting that $b$-value is proportional to pore-fluid pressure.

- $b$-value is proportional to depth below the reservoir, increasing from $b \sim 1.0$ at injection depth to $b \sim 1.5$, two kilometers below.

Chapter 5:

- We calculated 982 focal mechanism solutions for Ngatamariki (205) and Rotokawa (777) from 2012-2015, exhibiting predominantly normal faulting, with a lesser number of strike-slip events;

- Stress inversions from kmeans clusters at Ngatamariki reveal a pure normalfaulting regime in southern Ngatamariki and an unconventional regime in the north in which no principal stress is vertical; 
- Discrepancies between stress results within the northern injection zone at Ngatamariki may be related to thermal cooling and contraction of the reservoir;

- Stress inversion results at Rotokawa indicate a uniform, normal-faulting stress regime across the seismically-active portion of the reservoir;

- Temporal variations in the stress ratio, $\nu$, from 0.9-0.2 near the main injection wells at Rotokawa over six months reflect changes in the shape of the cooled zone of the reservoir with time.

Below, we discuss the implications of these findings, focusing on each field individually.

\subsubsection{Ngatamariki}

The portion of this thesis pertaining to Ngatamariki represents the first detailed study of seismicity at the field. The Ngatamariki catalog analyzed in this thesis contains over 2500 precisely-located events with a median horizontal error or $\pm 173 \mathrm{~m}$ and median depth error of $\pm 184 \mathrm{~m}$, spanning nearly four years during initial field development and plant startup.

At each step in our analysis, the distinction between the northern and southern injection zones at Ngatamariki is emphasized. Although flow rates and wellhead pressures in each zone are similar, the seismic response of the reservoir is not. Seismicity in the north has both fewer events of $\mathrm{M}_{L}>2.0$ (albeit with identical $b$-values of 1.42 ) and a more oblate spatial footprint. Additionally, stress inversion results from focal mechanisms in the north reveal an unconventional stress state with no vertical principal axis, whereas stress results in the south show the expected normal-faulting regime with NE-SW-oriented $\mathrm{S}_{\text {Hmax }}$. The two zones of the reservoir are known to be geologically distinct Bignall, 2009; Chambefort et al., 2014) and this may help explain the differences in seismicity. The southern injection wells intersect a structure inferred to be related to the Aratiatia Fault Zone (Figure 6.1), a large, kilometers-long structure that can accommodate large ruptures. The north is highly-fractured and dominated by a tonalite intrusive body (below $\sim 2000 \mathrm{~m}$ bsl) of unknown spatial extent. We infer the higher $b$-value in the north to be a result of pervasive fracturing associated with the emplacement of the intrusive body, resulting in a higher concentration of fractures of shorter length and thereby limiting the likelihood of large-magnitude seismicity.

We infer the stress-state variation between the northern and southern injection zones to also be a result of the emplacement of the intrusive body. However, within the northern zone, stress field variations may also be a result of injection operations. We have detailed a scenario in Section 5.4.1, through which the shape of the cooled zone of the reservoir would preferentially decrease $\sigma_{1}$, thereby significantly altering the 

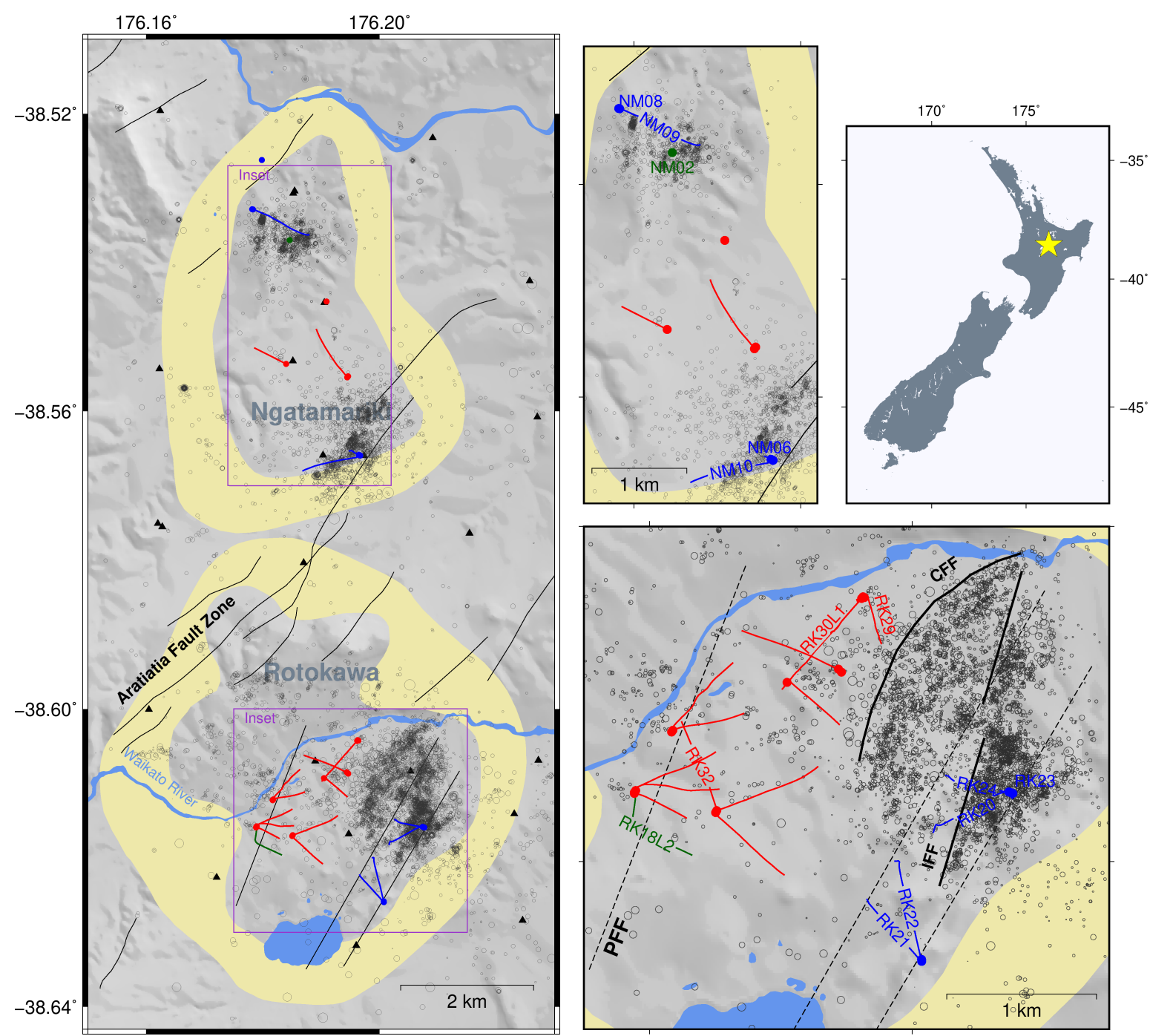

Figure 6.1: Overview of the combined, Ngatamariki-Rotokawa seismic catalog presented in this thesis. Yellow polygons define the outlines of the resources and black lines represent active faults (Langridge et al., 2016). Black circles are seismic events with the radius of each symbol proportional to the magnitude. Blue, red and green lines are injection, production and monitoring wells, respectively. In the Rotokawa inset (lower right panel) dotted lines represent the previously-modeled orientations of the Production Field Fault ( $(\overline{\mathrm{PFF}}), \mathrm{CFF}$ and IFF. The bold black lines indicate the modified orientations for the CFF and IFF inferred in this thesis from the location of seismicity.

reservoir stress state, as has been observed and modeled elsewhere (Martínez-Garzón et al., 2013; Jeanne et al., 2015a).

We have also conducted a detailed spatio-temporal analysis of seismicity during pre-startup drilling and stimulation operations at Ngatamariki. These results suggest that injectivity index, a proxy for permeability increase, during injection operations is poorly correlated with both the location and rate of associated seismicity (Chapter 3; Figure 3.5). This result agrees with recent work by Riffault et al. (2018) and Cappa et al. (2019), showing that $I I$ is not necessarily a result of seismic slip on preexisting 
fractures, but instead may be dominated by near-well, aseismic fracture slip/opening near the wellbore. We infer this to be the case during the stimulation of NM08 in northern Ngatamariki, where $I I$ increases throughout the treatment but seismicity is induced only after a week of injection. In addition, injection well NM09, which is seismically active after plant startup, induced no observed seismic events during pre-startup stimulation and injection testing. However, $I I$ increased throughout this treatment, leading to the simple conclusion that the observed permeability increase was due to aseismic slip or openning.

\subsubsection{Rotokawa}

Previous studies of Rotokawa seismicity by Sherburn et al. (2015) and Sewell et al. (2015b) identified an area of active seismicity (as of 2012) in the northeast part of the field, between the northern injection wells and northern production wells, extending NE towards the field boundary. The catalog presented here, from 2012-2015, shows a broadly similar seismically-active area. Where the catalog of Sherburn et al. (2015) and Sewell et al. (2015b) showed only a single microseismic cloud, our catalog is able to distinguish between different structures. From our high-precision locations of nearly 6500 events (median horizontal error: $186 \mathrm{~m}$; median depth error: $231 \mathrm{~m}$ ), we infer a modified location for the Central Field Fault (CFF), located northwest of its previouslydetermined location. We are also able to define the orientation of what we infer to be the Injection Field Fault (IFF) (Figure 6.1). This structure separates injection wells RK20 and RK24 from well RK23, as confirmed by vertical offsets in well cuttings, and extends northwestward, subparallel to the CFF.

While the rate of seismicity at Ngatamariki is well correlated with injection operations, no obvious correlation exists at Rotokawa. This is partially because the background rate of seismicity was already elevated at the start of our dataset, owing to Rotokawa's history of development dating to 1997. At Ngatamariki, the background rate of seismicity is effectively zero (relative to the rate of induced events), making injection-related rate changes easy to identify. Changes in seismicity at Rotokawa, if they exist, are more difficult to distinguish. It has been suggested that periods with an elevated rate of seismicity (termed 'swarm' events) correspond to periods during which the power plants are shut down for maintenance (Sewell et al., 2015b). However, we are not able to confirm such a correlation for our dataset, with equal numbers of 'swarms' occurring at times of normal operation and at times of plant shutdowns. Rate changes are equally difficult to identify for the major injection switch between RK23 and RK24, perhaps because this flow-rate change $(\sim 300 \mathrm{t} / \mathrm{h})$ is small when compared with those during startup at Ngatamariki $(\sim 1000 \mathrm{t} / \mathrm{h})$. We therefore attempt to correlate other measures of seismicity with injection operations at Rotokawa, including magnitude distributions and stress inversion parameters. 
Spatial mapping of $b$-values has been used to infer areas of elevated pore-fluid pressure at other geothermal projects (e.g. Bachmann et al., 2012). At Rotokawa, our catalog reveals a complex three-dimensional picture of $b$. These variations correlate with areas of elevated pressure. For example, in the southeast compartment of seismicity at Rotokawa, closest to the injection wells, $b$-value is significantly higher than in other seismically-active areas of the reservoir. However, $b$-value is also likely linked to reservoir properties such as fracture density and size, as well as temperature gradient (e.g. Wiemer et al. 1998). How these properties interact to produce the magnitudefrequency distribution for a specific point in the reservoir is an open research topic.

Stress inversions from fourteen clusters of events at Rotokawa indicate a purely normal-faulting regime throughout the reservoir. However, the recovered stress ratio, $\nu$, and direction of maximum horizontal compressive stress, $\mathrm{S}_{\text {Hmax }}$, vary in time and space. These variations may be related to anisotropic reservoir contraction due to the injection of cold fluid, with stress inversions for the events nearest the injection wells showing consistently lower stress ratios than those further afield. This interpretation is supported by a steep drop in stress ratio $(\sim 0.9$ to $\sim 0.2$ in six months; Equation 5.1) coinciding with the restart of injection well RK23. We interpret this to reflect the preferential reduction of $\sigma_{1}\left(\approx \sigma_{V}\right)$ as newly-injected, dense, cold fluid flows downwards before gradually transitioning to lateral flow.

\subsection{Prospects for further research}

\subsubsection{Mechanisms of matched-filter detected events}

We collect and analyze nearly 1000 earthquake focal mechanism solutions spanning four years. Although this would be considered a large number of focal mechanisms for some catalogs, the spatial and temporal density of these events is too low to allow detailed examination of reservoir fracture properties (see Chapter 5). In Chapters 3 and 4 we analyze the spatial and temporal patterns revealed in catalogs of matchedfilter detected and precisely-relocated seismicity. Few of the newly-detected (i.e. nontemplate) events have signal-to-noise ratios high enough to manually pick P-arrival polarities. However, researchers at Long Valley Caldera (Figure 6.2) and Yellowstone have developed waveform-correlation-based methods capable of determining the focal mechanism solutions of these detected events (Shelly et al., 2016b; Hotovec-Ellis et al., 2018; Shelly and Hardebeck, 2018).

This approach can be successfully applied to our Ngatamariki and Rotokawa catalogs, and may realistically increase the number of focal mechanism observations by an order of magnitude. Specifically, the patterns revealed by these focal mechanisms can provide a detailed understanding of the location and orientation of specific flow 

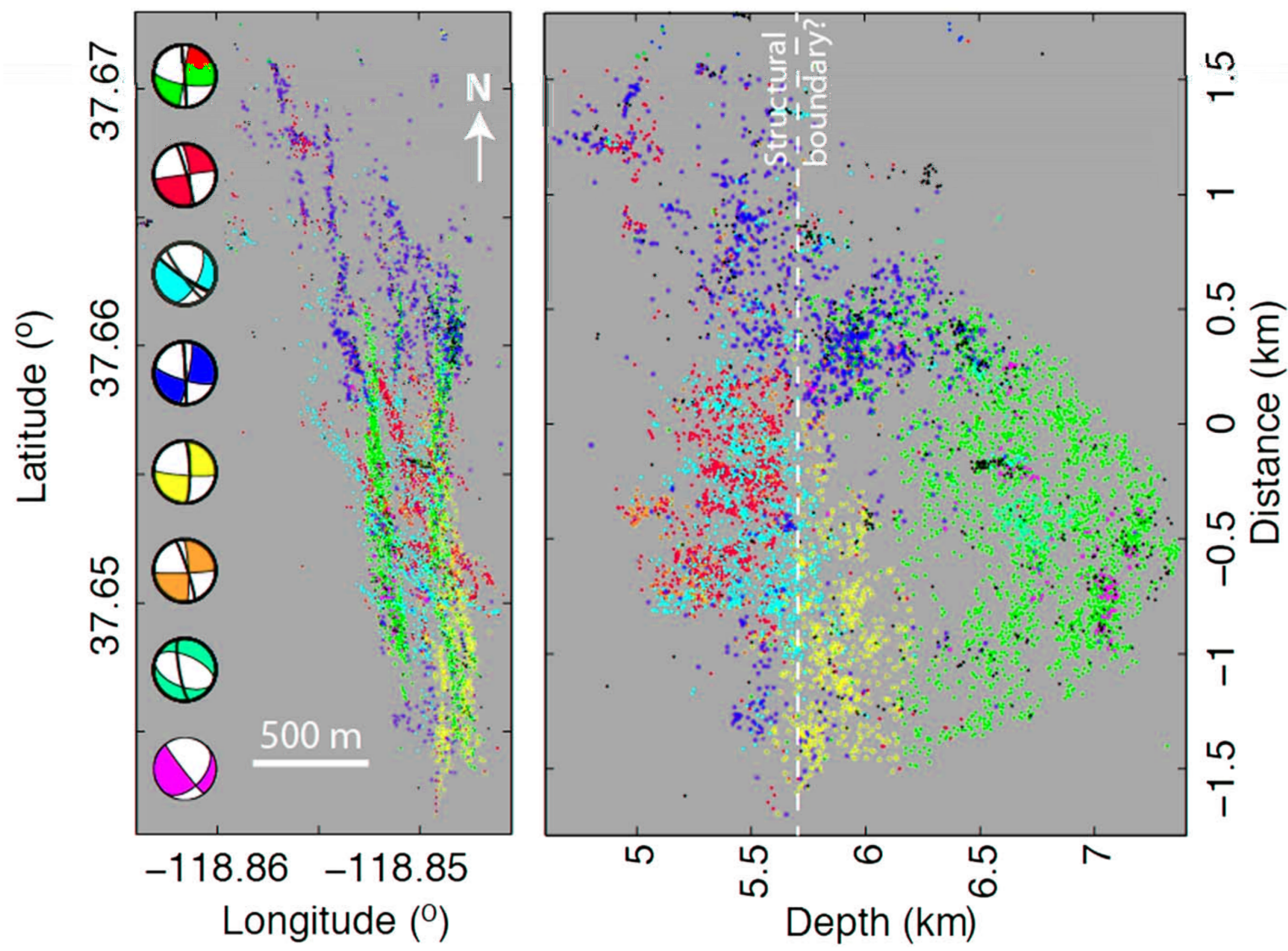

Figure 6.2: Figure of Shelly et al. (2016b) showing the spatial distribution of event clusters determined using a waveform-correlation-based polarity clustering technique. The focal mechanisms depicted to the left of the figure show the 'correlation composite' mechanism for each of the eight clusters. The locations of the events in each cluster, shown in map view and cross-section, are color-coded to match their corresponding focal mechanism.

pathways in the fields. Shelly et al. (2016b), Hotovec-Ellis et al. (2018) and Shelly and Hardebeck (2018) were able to reveal complex faulting patterns at a scale of $100 \mathrm{~s}$ of meters and in geologic settings similar to that of the TVZ, which suggests that we may also be able to implement this methodology at Rotokawa and Ngatamariki. In addition, the seismic network used in the aforementioned studies was more sparse than the network used here, which may bode well for the application of this methodology to our dataset. To this end, we suggest these techniques be applied to the Ngatamariki and Rotokawa catalogs.

\subsubsection{Thermo-hydro-mechanical modeling}

As discussed in Chapter 5, knowledge of the stress state within a geothermal reservoir is key to understanding which fractures and faults are critically-stressed, susceptible to slip and therefore more likely to serve as fluid flow pathways. We have made a case for the use of earthquake focal mechanism inversion as a tool for monitoring 
reservoir stresses at Rotokawa and Ngatamariki, but have little stress data with which to verify our results. While Davidson et al. (2012) and McNamara et al. (2015) have characterized certain aspects of the stress field at Rotokawa, the results are constrained only to certain wells in the aseismic production field and are therefore of limited use for constraining focal mechanism inversions located in a separate reservoir compartment.

Geothermal operators heavily rely on numerical reservoir modeling for projecting field performance and developing production/injection strategies Grant and Bixley. 2011; DiPippo, 2016). At a minimum, these models simulate heat and multi-phase fluid flow in fractured and porous media, but variations of the most popular software packages (e.g. TOUGH, FEHM) also integrate equations for mass transfer, allowing for the simulation of the coupled thermo-hydro-mechanical system (Zyvoloski, 2007; Jung et al. 2017). It is therefore possible to simulate the state of stress in a reservoir during injection and production, while taking into consideration reservoir contraction due to cooling, which we and others have suggested dominates the induced stress change within a geothermal reservoir (Stephens and Voight, 1982, Jeanne et al., 2014 , Martínez-Garzón et al., 2013, 2014; Jeanne et al., 2015a,b).

Simulating selected injection operations of interest at Rotokawa and Ngatamariki using these techniques would be valuable for assessing the mechanisms of stress change in the reservoir. Of particular interest are the northern injection wells (NM08, NM09) at Ngatamariki, due to the unexpected stress states observed from focal mechanism inversions in this part of the field. By simulating the stress variation due to cold water injection, one could assess if reservoir contraction is a realistic cause of stress change, or if the emplacement of the intrusive body is a more likely mechanism. For example, the stimulations of NM08 and NM09 could be simulated using the finite element program FEHM (Zyvoloski, 2007, Figure 6.3) and the effects of starting model parameters such as fracture spacing, permeability anisotropy and intrusive geometry on reservoir stress changes could be investigated.

Modeling at Rotokawa is more complicated than at Ngatamariki, due mostly to decades of production prior to our study period, which has had an unknown effect on the stress state in the reservoir. However, we have better temporal stress resolution from focal mechanism inversion at Rotokawa than Ngatamariki. As a result, it may be possible to compare the observed stress changes in the southeast compartment at Rotokawa with numerical modeling of wells RK20, 23 and 24. Specifically, future workers should look to answer the following questions regarding this section of the reservoir:

- Are the observed stress ratio changes (interpreted to be related to injection at RK23) realistic and, if so, for what initial conditions can our stress inversions be matched (i.e. fracture spacing, initial stress state)? 


\section{Final Temperatures: NM08 Stimulation}

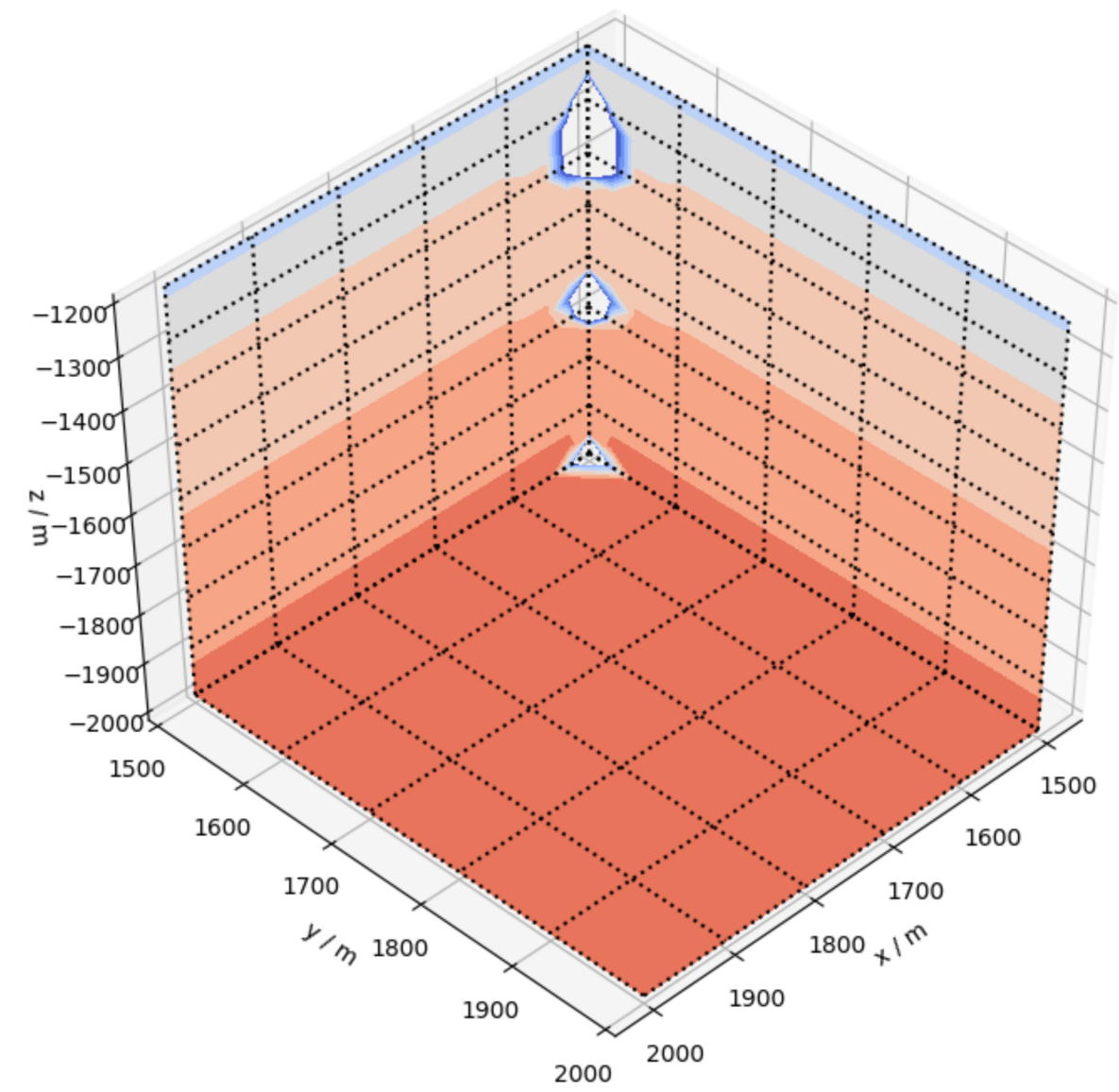

Figure 6.3: Example temperature output from finite element modeling software FEHM (Zyvoloski, 2007) for northern Ngatamariki, following the stimulation of NM08. The cooled zones correspond to the feedzones in the well as interpreted from PTS runs. The model shown here contains four flat-lying geologic units representing surface deposits, a low permeability clay cap, the Tahorakuri Formation and a tonalite intrusive body, with increasing depth, respectively. Lateral permeability is anisotropic, and greater in the ' $y$ ' direction than the ' $x$ ' in order to simulate increased permeability in subparallel to $\mathrm{S}_{\text {Hmax }}$. This rather basic model could be extended to include faults (inferred from earthquake hypocenters) and additional wells, such as NM09. Pressure and temperature data from monitoring well NM02 could also be used to constrain the results of the simulation. Ideally, the modeled stresses would be compared to the stress inversion results for the kmeans clusters presented in Chapter 5.

- Does the Injection Field Fault (IFF) act as a flow barrier between RK23 and RK20/24? Is it necessary to explain the observed variations in stress, or can those variations be modeled for the case where all three wells are hydraulically connected?

- Are the observed variations in $b$-value at Rotokawa reflected in the simulated pressure distributions? Specifically, are areas of high pore-fluid pressure similar to areas of high b-value as has been suggested elsewhere (Bachmann et al., 2012)? 
Must other variables (e.g. fracture density/orientation) be included to explain $b$ value change in the reservoir?

\subsection{Concluding statement}

This thesis expands upon an automatically-processed earthquake catalog for the Rotokawa and Ngatamariki geothermal fields in New Zealand, doubling the number of events and decreasing the hypocentral uncertainties. We relate the properties of this precisely-located catalog to changes in injection operations at both fields, including the start-up of the Ngatamariki power plant and major injection migrations at Rotokawa. This is the first work of its kind at Ngatamariki, providing a baseline against which future seismicity can be measured. At Rotokawa we update the orientation of important flow-bounding faults and provide evidence for pressure-compartmentalization of the injection field. The work provided herein will serve to inform reservoir modeling at Rotokawa and Ngatamariki and further the understanding of the relationship between geothermal power production and seismicity more generally. 
Appendices 



\section{A Alternative detection techniques}

\section{A.1 Subspace Detection}

Although not incorporated into the results presented in the preceding chapters, we have developed and implemented functionality for additional detection techniques. Specifically, we have developed functionality for the use of 'subspace detection' of repeating and near-repeating seismicity, which we summarize below.

Matched-filter detection techniques are computationally expensive when applied across large datasets. The expense is multiplied as more earthquakes are added to the list of template events (referred to by Barrett and Beroza (2014) as a 'design set') and as larger seismograph networks with more channels of data are used. At the same time, it is important that a set of template events effectively represent the variety of sources present in a given study area or risk missing what might be important seismicity. Subspace detection has been used to address these considerations by expressing clusters of similar events as single matrices known as subspace detectors (for example Harris, 2006; Harris and Paik, 2006; Barrett and Beroza, 2014; Chambers et al., 2015).

\section{A.1.1 Subspace detector design}

As outlined by Harris (2006), there are five main steps in constructing a series of subspace detectors from a catalog of earthquakes:

1. Generate a distance matrix for all events by calculating, pairwise, the correlations between events in the catalog. Each element of the distance matrix, $D_{x, y}$ is equivalent to $1-R(x, y)$ where $R(x, y)$ is the cross correlation value between events $x$ and $y$;

2. Cluster the events in the catalog based on this distance matrix. Harris (2006) suggest a single-link agglomerative hierarchical clustering algorithm. Each cluster is then referred to as the design set for a single subspace detector;

3. For each cluster, align the waveforms by cross correlation and trim the waveforms such that the first arrival occurs at the same point for each trace. Each waveform defines a column, $i$, of the data vector, $\underline{X}$;

4. Calculate the orthonormal basis, $\underline{W}$, for the space defined by the design set, $\underline{X}$ : 


$$
\underline{X}=\underline{W \Sigma V^{T}}
$$

5. Determine a satisfactory dimension $d$ such that the subspace defined by $\underline{W}_{d}$ approximates the waveforms in the design set:

$$
\underline{X} \sim \underline{W}_{d} \underline{\Sigma}_{d} \underline{V}_{d}^{T}
$$

$W_{d}$ is our subspace detector and the matrix:

$$
\underline{A}_{d}=\underline{\Sigma}_{d} \underline{V}_{d}^{T}
$$

gives the relative importance of each dimension, $d$, of $\underline{W}_{d}$ in describing the design set, $\underline{X}$.

\section{A.1.2 Detection statistic}

Following the notation above, the detection statistic is defined as the following:

$$
c[n]=\frac{\underline{x}_{p}^{T}[n] \underline{x}_{p}[n]}{\underline{x}^{T}[n] \underline{x}[n]}
$$

where $\underline{x}[n]$ is the data vector within the moving detection window and $\underline{x}_{p}[n]$ is the projection of $\underline{x}[n]$ onto the space defined by the subspace detector such that:

$$
\underline{x}_{p}[n]=\underline{W}_{d} \underline{W}_{d}^{T} \underline{x}[n]
$$

This defines the ratio of the energy in the projected data $x_{p}[n]$ to the energy in the original data, $x[n]$ within the detection window $[n]$, which moves through the data period of interest one sample at a time.

\section{A.1.3 Determining a sufficient dimension}

Typically, the necessary number of dimensions in a subspace detector is determined empirically, using a plot such as Figure A.1, which shows the percentage of energy captured by the detector for all design set events with increasing dimensionality. For instance, Chambers et al. (2015) stipulate that the desired dimension, $d$, is the smallest dimension for which the subspace detector, $\underline{W}_{d}$ captures an average of $90 \%$ of the energy for all events in the design set, $\underline{X}$. 


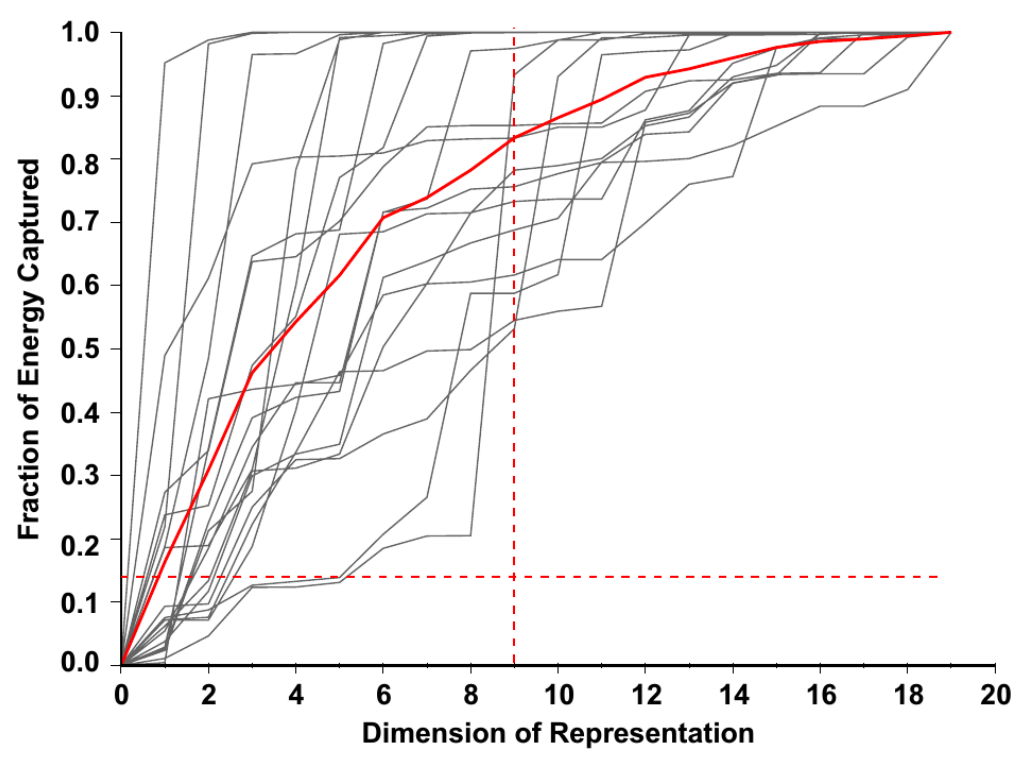

Figure A.1: Adapted from Harris et al. (2006), showing the percentage energy capture by a subspace detector with increasing dimensionality. Gray lines show energy capture for each event in the design set used to construct the detector and the solid red line shows the average across all events. The dotted red lines indicate the dimension $(n=9)$ corresponding to an average energy capture of $90 \%$.

\section{A.2 EQcorrscan development}

The functionality detailed above comprises the majority of my contribution to the open-source Python package, EQcorrscan (Chamberlain et al., 2017b), which is developed and maintained by VUW postdoctoral fellow Calum Chamberlain. The purpose of EQcorrscan is to provide an easy-to-use, fully-tested framework for the detection of repeating and near-repeating seismicity. The original development of the package centered around the matched-filter detection routine detailed in Chapter 2 but was expanded to include subspace detection during the course of this thesis. 


\section{Bibliography}

Abbott, B. P., et al., Properties of the Binary Black Hole Merger GW150914, Physical Review Letters, 116(24), doi:10.1103/physrevlett.116.241102, 2016.

Addison, S., J. Winick, B. Mountain, and F. Siega, Rotokawa reservoir tracer test history, in Proc. NZ Geothermal Workshop, 2015.

Addison, S., L. Sirad-Azwar, J. Clearwater, D. Hernandez, B. Mountain, A. Blair, and P. Siratovich, Improving the conceptual understanding through a recent injection of $200 \mathrm{GBq}$ of iodine-125 at the Rotokawa geothermal field, New Zealand, in 42nd Workshop on Geothermal Reservoir Engineering, 2017.

Aki, K., Maximum likelihood estimate of $\mathrm{b}$ in the formula $\log \mathrm{N}=\mathrm{a}-\mathrm{bM}$ and its confidence limits, Bull. Earthq. Res. Inst., Tokyo Univ., 43, 237-239, 1965.

Allen, R. V., Automatic earthquake recognition and timing from single traces, Bulletin of the Seismological Society of America, 68(5), 1521-1532, 1978.

Allis, R. G., Mechanism of induced seismicity at the Geysers Geothermal Reservoir California, Geophysical Research Letters, 9(6), 629-632, doi:10.1029/g1009i006p00629, 1982.

Allis, R. G., Review of subsidence at Wairakei field New Zealand, Geothermics, 29(4-5), 455-478, doi:10.1016/s0375-6505(00)00016-x, 2000.

Allmendinger, R. W., N. Cardozo, and D. M. Fisher, Structural Geology Algorithms: Vectors and Tensors, Cambridge University Press, 2011.

Álvarez-Gómez, J. A., Fmc: a one-liner python program to manage, classify and plot focal mechanisms, in EGU General Assembly Conference Abstracts, vol. 16, 2014.

Amann, F., V. Gischig, K. Evans, J. Doetsch, R. Jalali, B. Valley, H. Krietsch, N. Dutler, L. Villiger, B. Brixel, M. Klepikova, A. Kittilä, C. Madonna, S. Wiemer, M. O. Saar, S. Loew, T. Driesner, H. Maurer, and D. Giardini, The seismo-hydromechanical behavior during deep geothermal reservoir stimulations: Open questions tackled in a decameter-scale in situ stimulation experiment, Solid Earth, 9(1), 115-137, doi: 10.5194/se-9-115-2018, 2018. 
Arnold, R., and J. Townend, A Bayesian approach to estimating tectonic stress from seismological data, Geophysical Journal International, 170(3), 1336-1356, doi:10. 1111/j.1365-246x.2007.03485.x, 2007.

Aster, R., and C. Rowe, Automatic phase pick refinement and similar event association in large seismic datasets, in Advances in seismic event location, pp. 231-263, Springer, 2000.

Atkinson, G. M., D. W. Eaton, H. Ghofrani, D. Walker, B. Cheadle, R. Schultz, R. Shcherbakov, K. Tiampo, J. Gu, R. M. Harrington, Y. Liu, M. van der Baan, and H. Kao, Hydraulic Fracturing and Seismicity in the Western Canada Sedimentary Basin, Seismological Research Letters, 87(3), 631-647, doi:10.1785/0220150263, 2016.

Bachmann, C. E., S. Wiemer, B. P. Goertz-Allmann, and J. Woessner, Influence of pore-pressure on the event-size distribution of induced earthquakes, Geophysical Research Letters, 39(9), doi:10.1029/2012gl051480, 2012.

Baisch, S., R. Weidler, R. Voros, D. Wyborn, and L. de Graaf, Induced Seismicity during the Stimulation of a Geothermal HFR Reservoir in the Cooper Basin Australia, Bulletin of the Seismological Society of America, 96(6), 2242-2256, doi: 10.1785/0120050255, 2006.

Baisch, S., R. Vörös, E. Rothert, H. Stang, R. Jung, and R. Schellschmidt, A numerical model for fluid injection induced seismicity at Soultz-sous-Forêts, International Journal of Rock Mechanics and Mining Sciences, 47(3), 405-413, doi: 10.1016/j.ijrmms.2009.10.001, 2010.

Baria, R., A. Green, and R. Jones, Anomalous seismic events observed at the CSM HDR project U.K., International Journal of Rock Mechanics and Mining Sciences 86 Geomechanics Abstracts, 26(3-4), 257-269, doi:10.1016/0148-9062(89)91975-x, 1989.

Barrett, S. A., and G. C. Beroza, An Empirical Approach to Subspace Detection, Seismological Research Letters, 85(3), 594-600, doi:10.1785/0220130152, 2014.

Batini, F., R. Console, and G. Luongo, Seismological study of Larderello - Travale geothermal area, Geothermics, 14 (2-3), 255-272, doi:10.1016/0375-6505(85)90066-5, 1985 .

Bauer, S. J., and J. Handin, Thermal expansion and cracking of three confined watersaturated igneous rocks to $800^{\circ} \mathrm{C}$, Rock Mechanics and Rock Engineering, 16(3), 181-198, doi:10.1007/bf01033279, 1983.

Bertani, R., Geothermal power generation in the world 2010-2014 update report, Geothermics, 60, 31-43, doi:10.1016/j.geothermics.2015.11.003, 2016. 
Bibby, H., T. Caldwell, F. Davey, and T. Webb, Geophysical evidence on the structure of the Taupo Volcanic Zone and its hydrothermal circulation, Journal of Volcanology and Geothermal Research, 68(1-3), 29-58, doi:10.1016/0377-0273(95)00007-h, 1995.

Bignall, G., Ngatamariki geothermal field geoscience overview, GNS Science consultancy report 2009, 94, 35, 2009.

Biot, M. A., Theory of Propagation of Elastic Waves in a Fluid-Saturated Porous Solid. I. Low-Frequency Range, The Journal of the Acoustical Society of America, 28(2), 168-178, doi:10.1121/1.1908239, 1956.

Blackwell, D. D., P. T. Negraru, and M. C. Richards, Assessment of the Enhanced Geothermal System Resource Base of the United States, Natural Resources Research, 15(4), 283-308, doi:10.1007/s11053-007-9028-7, 2007.

Blake, K., and N. C. Davatzes, Crustal stress heterogeneity in the vicinity of coso geothermal field, ca, in Proceedings, Thirty-Sixth Workshop on Geothermal Reservoir Engineering, p. 11, 2011.

Boettcher, M. S., A. McGarr, and M. Johnston, Extension of gutenberg-richter distribution to mw- 1.3, no lower limit in sight, Geophysical Research Letters, 36(10), 2009.

Bohnhoff, M., S. Baisch, and H.-P. Harjes, Fault mechanisms of induced seismicity at the superdeep German Continental Deep Drilling Program (KTB) borehole and their relation to fault structure and stress field, Journal of Geophysical Research: Solid Earth, 109(B2), 2004.

Boseley, C., W. Cumming, L. Urzúa-Monsalve, T. Powell, and M. Grant, A resource conceptual model for the Ngatamariki geothermal field based on recent exploration well drilling and 3D MT resistivity imaging, in Proceedings World Geothermal Congress, 2010.

Bott, M. H. P., The mechanics of oblique slip faulting, Geological Magazine, 96(2), 109-117, 1959.

Boyle, K., and M. Zoback, The Stress State of the Northwest Geysers California Geothermal Field, and Implications for Fault-Controlled Fluid Flow, Bulletin of the Seismological Society of America, 104 (5), 2303-2312, doi:10.1785/0120130284, 2014.

Bromley, C., K. Brockbank, T. Glynn-Morris, M. Rosenberg, M. Pender, M. O'Sullivan, and S. Currie, Geothermal subsidence study at Wairakei-Tauhara New Zealand, Proceedings of the Institution of Civil Engineers - Geotechnical Engineering, 166(2), 211-223, doi:10.1680/geng.12.00040, 2013. 
Buscarlet, E., H. Moon, I. Wallis, and J. Quinao, Reservoir tracer test at the Ngatamariki Geothermal Field, in Proceedings 37th New Zealand Geothermal Workshop, vol. 18, p. 20, 2015.

Cant, J. L., P. A. Siratovich, J. W. Cole, M. C. Villeneuve, and B. M. Kennedy, Matrix permeability of reservoir rocks Ngatamariki geothermal field, Taupo Volcanic Zone, New Zealand, Geothermal Energy, 6(1), doi:10.1186/s40517-017-0088-6, 2018.

Cappa, F., M. M. Scuderi, C. Collettini, Y. Guglielmi, and J.-P. Avouac, Stabilization of fault slip by fluid injection in the laboratory and in situ, Science Advances, 5(3), eaau4065, 2019.

Carder, D. S., Seismic investigations in the Boulder Dam area, 1940-1944, and the influence of reservoir loading on local earthquake activity, Bulletin of the Seismological Society of America, 35(4), 175-192, 1945.

Castellazzi, C., M. K. Savage, E. Walsh, and R. Arnold, Shear wave automatic picking and splitting measurements at Ruapehu volcano New Zealand, Journal of Geophysical Research: Solid Earth, 120(5), 3363-3384, doi:10.1002/2014jb011585, 2015.

Catalli, F., A. P. Rinaldi, V. Gischig, M. Nespoli, and S. Wiemer, The importance of earthquake interactions for injection-induced seismicity: Retrospective modeling of the Basel Enhanced Geothermal System, Geophysical Research Letters, 43(10), 4992-4999, doi:10.1002/2016gl068932, 2016.

Chambefort, I., B. Lewis, C. Wilson, A. Rae, C. Coutts, G. Bignall, and T. Ireland, Stratigraphy and structure of the Ngatamariki geothermal system from new zircon $\mathrm{U}-\mathrm{Pb}$ geochronology: Implications for Taupo Volcanic Zone evolution, Journal of Volcanology and Geothermal Research, 274, 51-70, doi:10.1016/j.jvolgeores.2014.01. 015, 2014.

Chambefort, I., E. Buscarlet, I. C. Wallis, S. Sewell, and M. Wilmarth, Ngatamariki Geothermal Field New Zealand: Geology, geophysics, chemistry and conceptual model, Geothermics, 59, 266-280, doi:10.1016/j.geothermics.2015.07.011, 2016.

Chamberlain, C., and C. Hopp, EQcorrscan: EQcorrscan v 0.1.3, doi:10.5281/zenodo. 59976, 2016.

Chamberlain, C. J., D. R. Shelly, J. Townend, and T. A. Stern, Low-frequency earthquakes reveal punctuated slow slip on the deep extent of the Alpine Fault New Zealand, Geochemistry, Geophysics, Geosystems, 15(7), 2984-2999, doi:10.1002/ $2014 \mathrm{gc005436,} 2014$. 
Chamberlain, C. J., C. M. Boese, and J. Townend, Cross-correlation-based detection and characterisation of microseismicity adjacent to the locked late-interseismic Alpine Fault, South Westland, New Zealand, Earth and Planetary Science Letters, 457, 63-72, doi:10.1016/j.epsl.2016.09.061, 2017a.

Chamberlain, C. J., C. J. Hopp, C. M. Boese, E. Warren-Smith, D. Chambers, S. X. Chu, K. Michailos, and J. Townend, EQcorrscan: Repeating and Near-Repeating Earthquake Detection and Analysis in Python, Seismological Research Letters, 89 (1), 173-181, doi:10.1785/0220170151, 2017b.

Chambers, D. J., K. D. Koper, K. L. Pankow, and M. K. McCarter, Detecting and characterizing coal mine related seismicity in the Western U.S. using subspace methods, Geophysical Journal International, 203(2), 1388-1399, doi:10.1093/gji/ggv383, 2015.

Cichowicz, A., An automatic s-phase picker, Bulletin of the Seismological Society of America, 83(1), 180-189, 1993.

Clearwater, J., L. Azwar, M. Barnes, I. Wallis, and R. Holt, Changes in injection well capacity during testing and plant start-up at Ngatamariki, in World Geothermal Congress, 2015.

Cole, B., and H. Legmann, The Rotokawa Geothermal Project-A High Pressure, Sustainable and Environmentally Benign Power Plant, TRANSACTIONSGEOTHERMAL RESOURCES COUNCIL, pp. 509-514, 1998.

Cole, J., and K. Lewis, Evolution of the Taupo-Hikurangi subduction system, Tectonophysics, 72(1-2), 1-21, doi:10.1016/0040-1951(81)90084-6, 1981.

Cornet, F. H., Seismic and aseismic motions generated by fluid injections, Geomechanics for Energy and the Environment, 5, 42-54, doi:10.1016/j.gete.2015.12.003, 2016.

Das, I., and M. D. Zoback, Long-period long-duration seismic events during hydraulic fracture stimulation of a shale gas reservoir, The Leading Edge, 30(7), 778-786, doi:10.1190/1.3609093, 2011.

Davidson, J., P. Siratovich, I. Wallis, D. Gravley, and D. McNamara, Quantifying the stress distribution at the Rotokawa geothermal field, New Zealand, in Proceedings 34th New Zealand Geothermal Workshop, 2012.

Deichmann, N., and M. Garcia-Fernandez, Rupture geometry from high-precision relative hypocentre locations of microearthquake clusters, Geophysical Journal International, $110(3), 501-517,1992$. 
Deichmann, N., and D. Giardini, Earthquakes Induced by the Stimulation of an Enhanced Geothermal System below Basel (Switzerland), Seismological Research Letters, 80(5), 784-798, doi:10.1785/gssrl.80.5.784, 2009.

DeMets, C., R. G. Gordon, D. F. Argus, and S. Stein, Effect of recent revisions to the geomagnetic reversal time scale on estimates of current plate motions, Geophysical Research Letters, 21(20), 2191-2194, doi:10.1029/94gl02118, 1994.

Dempsey, D., S. Kelkar, N. Davatzes, S. Hickman, and D. Moos, Numerical modeling of injection stress and permeability enhancement during shear stimulation at the Desert Peak Enhanced Geothermal System, International Journal of Rock Mechanics and Mining Sciences, 78, 190-206, doi:10.1016/j.ijrmms.2015.06.003, 2015.

Diehl, T., N. Deichmann, E. Kissling, and S. Husen, Automatic S-Wave picker for local earthquake tomography, Bulletin of the Seismological Society of America, 99(3), 1906-1920, doi:10.1785/0120080019, 2009.

Dinske, C., and S. A. Shapiro, Seismotectonic state of reservoirs inferred from magnitude distributions of fluid-induced seismicity, Journal of Seismology, 17(1), 13-25, doi:10.1007/s10950-012-9292-9, 2012.

DiPippo, R., Geothermal Power Generation, Elsevier, doi:https://doi.org/10.1016/ C2014-0-03384-9, 2016.

Dodge, D. A., and W. R. Walter, Initial Global Seismic Cross-Correlation Results: Implications for Empirical Signal Detectors, Bulletin of the Seismological Society of America, 105(1), 240-256, doi:10.1785/0120140166, 2015.

Dodge, D. A., G. C. Beroza, and W. Ellsworth, Detailed observations of california foreshock sequences: Implications for the earthquake initiation process, Journal of Geophysical Research: Solid Earth, 101 (B10), 22,371-22,392, 1996.

Downs, D., J. Rowland, C. Wilson, M. Rosenberg, G. Leonard, and A. Calvert, Evolution of the intra-arc Taupo-Reporoa Basin within the Taupo Volcanic Zone of New Zealand, Geosphere, 10(1), 185-206, 2014.

Ellsworth, W. L., Injection-Induced Earthquakes, Science, 341(6142), 1225,9421225,942, doi:10.1126/science.1225942, 2013.

Esaki, T., S. Du, Y. Mitani, K. Ikusada, and L. Jing, Development of a shear-flow test apparatus and determination of coupled properties for a single rock joint, International Journal of Rock Mechanics and Mining Sciences, 36(5), 641-650, doi: 10.1016/s0148-9062(99)00044-3, 1999. 
Evans, K. F., H. Moriya, H. Niitsuma, R. H. Jones, W. S. Phillips, A. Genter, J. Sausse, R. Jung, and R. Baria, Microseismicity and permeability enhancement of hydrogeologic structures during massive fluid injections into granite at $3 \mathrm{~km}$ depth at the Soultz HDR site, Geophysical Journal International, 160(1), 389-412, doi: 10.1111/j.1365-246x.2004.02474.x, 2004.

Evans, K. F., A. Genter, and J. Sausse, Permeability creation and damage due to massive fluid injections into granite at $3.5 \mathrm{~km}$ at Soultz: 1. Borehole observations, Journal of Geophysical Research: Solid Earth, 110(B4), doi:10.1029/2004jb003168, 2005.

Evans, K. F., A. Zappone, T. Kraft, N. Deichmann, and F. Moia, A survey of the induced seismic responses to fluid injection in geothermal and $\mathrm{CO} 2$ reservoirs in Europe, Geothermics, 41, 30-54, doi:10.1016/j.geothermics.2011.08.002, 2012.

Fang, Y., C. Wang, D. Elsworth, and T. Ishibashi, Seismicity-permeability coupling in the behavior of gas shales CO2 storage and deep geothermal energy, Geomechanics and Geophysics for Geo-Energy and Geo-Resources, 3(2), 189-198, doi: 10.1007/s40948-017-0051-9, 2017.

Fang, Y., D. Elsworth, and T. T. Cladouhos, Reservoir permeability mapping using microearthquake data, Geothermics, 72, 83-100, doi:10.1016/j.geothermics.2017.10. 019, 2018.

Fehler, M., L. House, W. Phillips, and R. Potter, A method to allow temporal variation of velocity in travel-time tomography using microearthquakes induced during hydraulic fracturing, Tectonophysics, 289(1-3), 189-201, doi:10.1016/s0040-1951(97) 00315-6, 1998.

Feng, Q., and J. M. Lees, Microseismicity, stress, and fracture in the Coso geothermal field, California, Tectonophysics, 289(1-3), 221-238, 1998.

Frémont, M.-J., and S. D. Malone, High precision relative locations of earthquakes at mount st. helens, washington, Journal of Geophysical Research: Solid Earth, 92(B10), 10,223-10,236, 1987.

Garcia, J., C. Hartline, M. Walters, M. Wright, J. Rutqvist, P. F. Dobson, and P. Jeanne, The Northwest Geysers EGS Demonstration Project California, Geothermics, 63, 97-119, doi:10.1016/j.geothermics.2015.08.003, 2016.

Garrison, L. E., Geothermal Steam in The Geysers-Clear Lake Region California, Geological Society of America Bulletin, 83(5), 1449, doi:10.1130/0016-7606(1972) 83[1449:gsitgl]2.0.co;2, 1972. 
Ge, S., W. Ellsworth, D. Giardini, J. Townend, and T. Shimamoto, Overseas research advisory committee report on the pohang earthquake, Tech. rep., Korean Government Commission on the Cause of the Pohang Earthquake: Overseas Research Advisory Committee, 2019.

Geiger, L., Herbsetimmung bei Erdbeben aus den Ankunfzeiten, K. Gessell. Wiss. Goett., 4, 331-349, 1910.

Gephart, J. W., and D. W. Forsyth, An improved method for determining the regional stress tensor using earthquake focal mechanism data: application to the san fernando earthquake sequence, Journal of Geophysical Research: Solid Earth, 89(B11), 93059320, 1984.

Ghidaoui, M. S., M. Zhao, D. A. McInnis, and D. H. Axworthy, A Review of Water Hammer Theory and Practice, Applied Mechanics Reviews, 58(1), 49, doi:10.1115/ $1.1828050,2005$.

Gibbons, S. J., and F. Ringdal, The detection of low magnitude seismic events using array-based waveform correlation, Geophysical Journal International, 165(1), 149166, doi:10.1111/j.1365-246x.2006.02865.x, 2006.

Gibbons, S. J., and F. Ringdal, Seismic Monitoring of the North Korea Nuclear Test Site Using a Multichannel Correlation Detector, IEEE Transactions on Geoscience and Remote Sensing, 50(5), 1897-1909, doi:10.1109/tgrs.2011.2170429, 2012.

Goebel, T., M. Weingarten, X. Chen, J. Haffener, and E. Brodsky, The 2016 Mw5.1 Fairview Oklahoma earthquakes: Evidence for long-range poroelastic triggering at $40 \mathrm{~km}$ from fluid disposal wells, Earth and Planetary Science Letters, 472, 50-61, doi:10.1016/j.epsl.2017.05.011, 2017.

Goebel, T. H. W., and E. E. Brodsky, The spatial footprint of injection wells in a global compilation of induced earthquake sequences, Science, 361 (6405), 899-904, doi:10.1126/science.aat5449, 2018.

Goertz-Allmann, B. P., S. J. Gibbons, V. Oye, R. Bauer, and R. Will, Characterization of induced seismicity patterns derived from internal structure in event clusters, Journal of Geophysical Research: Solid Earth, 122(5), 3875-3894, doi: 10.1002/2016jb013731, 2017.

Got, J.-L., J. Fréchet, and F. W. Klein, Deep fault plane geometry inferred from multiplet relative relocation beneath the south flank of kilauea, Journal of Geophysical Research: Solid Earth, 99 (B8), 15,375-15,386, 1994.

Grant, M. A., and P. F. Bixley, Geothermal Reservoir Engineering, Elsevier, doi:https: //doi.org/10.1016/C2010-0-64792-4, 2011. 
Grant, M. A., J. Clearwater, J. Quinão, P. F. Bixley, and M. Le Brun, Thermal stimulation of geothermal wells: A review of field data, in Thirty-Eighth Workshop on Geothermal Reservoir Engineering, 2013.

Gregg, D. R., Natural heat flow from the thermal areas of Taupo Sheet District (N 94), New Zealand Journal of Geology and Geophysics, 1(1), 65-75, doi:10.1080/00288306. 1958.10422795, 1958.

Grigoli, F., S. Cesca, A. P. Rinaldi, A. Manconi, J. A. López-Comino, J. F. Clinton, R. Westaway, C. Cauzzi, T. Dahm, and S. Wiemer, The November 2017Mw5.5 Pohang earthquake: A possible case of induced seismicity in South Korea, Science, 360 (6392), 1003-1006, doi:10.1126/science.aat2010, 2018.

Guglielmi, Y., F. Cappa, J.-P. Avouac, P. Henry, and D. Elsworth, Seismicity triggered by fluid injection-induced aseismic slip, Science, 348(6240), 1224-1226, doi:10.1126/ science.aab0476, 2015.

Gupta, H. K., Review: Reservoir Triggered Seismicity (RTS) at Koyna India, over the Past 50 Yrs, Bulletin of the Seismological Society of America, 108(5B), 2907-2918, doi:10.1785/0120180019, 2018.

Gutenberg, B., and C. F. Richter, Earthquake magnitude, intensity, energy, and acceleration, Bulletin of the Seismological Society of America, 32(3), 163-191, 1942.

Halwa, L., FMI Image Interpretation Report: Might River Power Ngatamariki-9, Tech. rep., Schlumberger Oilfield Australia, 2013a.

Halwa, L., Wellbore Image Interpretation Results NM10 (FMI \& AFIT - Final), Tech. rep., Schlumberger Oilfield Australia, 2013b.

Hanks, T. C., and H. Kanamori, A moment magnitude scale, Journal of Geophysical Research, 84(B5), 2348, doi:10.1029/jb084ib05p02348, 1979.

Hardebeck, J., and P. Shearer, A New Method for Determining First-Motion Focal Mechanisms, Bulletin of the Seismological Society of America, 92(6), 2264-2276, doi:10.1785/0120010200, 2002.

Hardebeck, J. L., and A. J. Michael, Stress orientations at intermediate angles to the san andreas fault, california, Journal of Geophysical Research: Solid Earth, 109(B11), 2004.

Hardebeck, J. L., and A. J. Michael, Damped regional-scale stress inversions: Methodology and examples for southern california and the coalinga aftershock sequence, Journal of Geophysical Research: Solid Earth, 111(B11), 2006. 
Häring, M. O., U. Schanz, F. Ladner, and B. C. Dyer, Characterisation of the basel 1 enhanced geothermal system, Geothermics, 37(5), 469-495, 2008.

Harris, D. B., Subspace Detectors: Theory, Tech. rep., Lawrence Livermore National Laboratory (LLNL) Livermore, CA, doi:10.2172/900081, 2006.

Harris, D. B., and T. Paik, Subspace Detectors: Efficient Implementation, Tech. rep., Lawrence Livermore National Laboratory (LLNL) Livermore, CA, doi:10.2172/ 898451, 2006.

Hartigan, J. A., Clustering algorithms, 1975.

Healy, J. H., W. W. Rubey, D. T. Griggs, and C. B. Raleigh, The Denver Earthquakes, Science, 161 (3848), 1301-1310, doi:10.1126/science.161.3848.1301, 1968.

Hernandez, D., S. Addison, S. Sewell, L. Azwar, and M. Barnes, Rotokawa: Reservoir Response of 172 MW Geothermal Operation, in Proceedings 37th New Zealand Geothermal Workshop, vol. 18, p. 20, 2015.

Hincks, T., W. Aspinall, R. Cooke, and T. Gernon, Oklahoma's induced seismicity strongly linked to wastewater injection depth, Science, 359(6381), 1251-1255, doi: 10.1126/science.aap7911, 2018.

Holcomb, D., General theory of the Kaiser effect, International Journal of Rock Mechanics and Mining Sciences \& Geomechanics Abstracts, 30(7), 929-935, doi: 10.1016/0148-9062(93)90047-h, 1993.

Hopp, C., S. Sewell, S. Mroczek, M. Savage, and J. Townend, Seismic response to injection well stimulation in a high-temperature, high-permeability reservoir, Geochemistry, Geophysics, Geosystems, 2019.

Horne, R., Modern Well Test Analysis, Petroway Inc., 1995.

Horton, S., Disposal of Hydrofracking Waste Fluid by Injection into Subsurface Aquifers Triggers Earthquake Swarm in Central Arkansas with Potential for Damaging Earthquake, Seismological Research Letters, 83(2), 250-260, doi:10.1785/gssrl. 83.2.250, 2012.

Hotovec-Ellis, A. J., D. R. Shelly, D. P. Hill, A. M. Pitt, P. B. Dawson, and B. A. Chouet, Deep fluid pathways beneath Mammoth Mountain California, illuminated by migrating earthquake swarms, Science Advances, 4(8), eaat5258, doi:10.1126/ sciadv.aat5258, 2018.

Hough, S. E., and M. Page, Potentially Induced Earthquakes during the Early Twentieth Century in the Los Angeles Basin, Bulletin of the Seismological Society of America, 106 (6), 2419-2435, doi:10.1785/0120160157, 2016. 
Hsieh, P. A., and J. D. Bredehoeft, A reservoir analysis of the Denver earthquakes: A case of induced seismicity, Journal of Geophysical Research: Solid Earth, 86(B2), 903-920, doi:10.1029/jb086ib02p00903, 1981.

Hurst, A., H. Bibby, and R. Robinson, Earthquake focal mechanisms in the central taupo volcanic zone and their relation to faulting and deformation, New Zealand journal of geology and geophysics, 45(4), 527-536, 2002.

Hurst, T., S. Bannister, R. Robinson, and B. Scott, Characteristics of three recent earthquake sequences in the taupo volcanic zone, new zealand, Tectonophysics, 452(1-4), 17-28, 2008.

Ishibashi, T., D. Elsworth, Y. Fang, J. Riviere, B. Madara, H. Asanuma, N. Watanabe, and C. Marone, Friction-stability-permeability evolution of a fracture in granite, Water Resources Research, doi:10.1029/2018wr022598, 2018.

Ito, A., High resolution relative hypocenters of similar earthquakes by cross-spectral analysis method, Journal of Physics of the Earth, 33(4), 279-294, 1985.

Jeanne, P., J. Rutqvist, P. F. Dobson, M. Walters, C. Hartline, and J. Garcia, The impacts of mechanical stress transfers caused by hydromechanical and thermal processes on fault stability during hydraulic stimulation in a deep geothermal reservoir, International Journal of Rock Mechanics and Mining Sciences, 72, 149-163, doi: 10.1016/j.ijrmms.2014.09.005, 2014.

Jeanne, P., J. Rutqvist, P. F. Dobson, J. Garcia, M. Walters, C. Hartline, and A. Borgia, Geomechanical simulation of the stress tensor rotation caused by injection of cold water in a deep geothermal reservoir, Journal of Geophysical Research: Solid Earth, 120(12), 8422-8438, doi:10.1002/2015jb012414, 2015a.

Jeanne, P., J. Rutqvist, A. P. Rinaldi, P. F. Dobson, M. Walters, C. Hartline, and J. Garcia, Seismic and aseismic deformations and impact on reservoir permeability: The case of EGS stimulation at The Geysers California, USA, Journal of Geophysical Research: Solid Earth, 120(11), 7863-7882, doi:10.1002/2015jb012142, 2015b.

Jung, Y., G. S. H. Pau, S. Finsterle, and R. M. Pollyea, Tough3: A new efficient version of the tough suite of multiphase flow and transport simulators, Computers $\&$ Geosciences, 108, 2-7, 2017.

Kanamori, H., Magnitude scale and quantification of earthquakes, Tectonophysics, 93(3-4), 185-199, doi:10.1016/0040-1951(83)90273-1, 1983.

Kanamori, H., N. Fujii, and H. Mizutani, Thermal diffusivity measurement of rockforming minerals from $300^{\circ}$ to $1100^{\circ} \mathrm{K}$, Journal of Geophysical Research, 73(2), 595605, doi:10.1029/jb073i002p00595, 1968. 
Kaverina, A., A. Lander, and A. Prozorov, Global creepex distribution and its relation to earthquake-source geometry and tectonic origin, Geophysical Journal International, 125(1), 249-265, 1996.

Keranen, K. M., and M. Weingarten, Induced seismicity, Annual Review of Earth and Planetary Sciences, 46, 149-174, 2018.

Keranen, K. M., H. M. Savage, G. A. Abers, and E. S. Cochran, Potentially induced earthquakes in Oklahoma USA: Links between wastewater injection and the 2011 Mw 5.7 earthquake sequence, Geology, 41 (6), 699-702, doi:10.1130/g34045.1, 2013.

Kim, K.-H., J.-H. Ree, Y. Kim, S. Kim, S. Y. Kang, and W. Seo, Assessing whether the 2017 Mw 5.4 Pohang earthquake in South Korea was an induced event, Science, 360(6392), 1007-1009, doi:10.1126/science.aat6081, 2018.

Kim, W.-Y., Induced seismicity associated with fluid injection into a deep well in Youngstown Ohio, Journal of Geophysical Research: Solid Earth, 118(7), 3506-3518, doi:10.1002/jgrb.50247, 2013.

Kim, W.-Y., and P. G. Richards, North Korean Nuclear Test: Seismic Discrimination at Low Yield, Eos Transactions American Geophysical Union, 88(14), 158, doi: 10.1029/2007eo140002, 2007.

Kissling, E., W. L. Ellsworth, D. Eberhart-Phillips, and U. Kradolfer, Initial reference models in local earthquake tomography, Journal of Geophysical Research: Solid Earth, 99(B10), 19,635-19,646, doi:10.1029/93jb03138, 1994.

Krischer, L., hypoDDpy: hypoDDpy 1.0, doi:10.5281/zenodo.18907, 2015.

Kwiatek, G., P. Martínez-Garzón, G. Dresen, M. Bohnhoff, H. Sone, and C. Hartline, Effects of long-term fluid injection on induced seismicity parameters and maximum magnitude in northwestern part of The Geysers geothermal field, Journal of Geophysical Research: Solid Earth, 120(10), 7085-7101, doi:10.1002/2015jb012362, 2015.

Langenbruch, C., and S. Shapiro, Gutenberg-richter relation originates from coulomb stress fluctuations caused by elastic rock heterogeneity, Journal of Geophysical Research: Solid Earth, 119(2), 1220-1234, 2014.

Langenbruch, C., and M. D. Zoback, How will induced seismicity in Oklahoma respond to decreased saltwater injection rates?, Science Advances, 2(11), e1601,542e1601,542, doi:10.1126/sciadv.1601542, 2016.

Langenbruch, C., and M. D. Zoback, Response to Comment on "How will induced seismicity in Oklahoma respond to decreased saltwater injection rates?", Science Advances, 3(8), eaao2277, doi:10.1126/sciadv.aao2277, 2017. 
Langenbruch, C., M. Weingarten, and M. D. Zoback, Physics-based forecasting of manmade earthquake hazards in Oklahoma and Kansas, Nature Communications, 9(1), doi:10.1038/s41467-018-06167-4, 2018.

Langridge, R., W. Ries, N. Litchfield, P. Villamor, R. V. Dissen, D. Barrell, M. Rattenbury, D. Heron, S. Haubrock, D. Townsend, J. Lee, K. Berryman, A. Nicol, S. Cox, and M. Stirling, The New Zealand Active Faults Database, New Zealand Journal of Geology and Geophysics, 59(1), 86-96, doi:10.1080/00288306.2015.1112818, 2016.

Lee, H. S., and T. F. Cho, Hydraulic Characteristics of Rough Fractures in Linear Flow under Normal and Shear Load, Rock Mechanics and Rock Engineering, 35(4), 299-318, doi:10.1007/s00603-002-0028-y, 2002.

Lee, W. H. K., and J. C. Lahr, HYPO71: A computer program for determining hypocenter magnitude, and first motion pattern of local earthquakes, doi: 10.3133/ofr72224, 1972.

Legmann, H., and P. Sullivan, The 30 MW Rotokawa I geothermal project five years of operation, in International Geothermal Conference, pp. 26-31, 2003.

Leonard, M., and B. Kennett, Multi-component autoregressive techniques for the analysis of seismograms, Physics of the Earth and Planetary Interiors, 113(1), 247-263, 1999.

Lomax, A., J. Virieux, P. Volant, and C. Berge-Thierry, Probabilistic Earthquake Location in 3D and Layered Models, in Advances in Seismic Event Location, pp. 101-134, Springer Netherlands, doi:10.1007/978-94-015-9536-0_5, 2000.

Lomax, A., A. Michelini, and A. Curtis, Earthquake Location Direct, Global-Search Methods, in Encyclopedia of Complexity and Systems Science, pp. 1-33, Springer New York, doi:10.1007/978-3-642-27737-5_150-2, 2014.

Lund, B., and J. Townend, Calculating horizontal stress orientations with full or partial knowledge of the tectonic stress tensor, Geophysical Journal International, $170(3)$, 1328-1335, doi:10.1111/j.1365-246x.2007.03468.x, 2007.

Majer, E. L., R. Baria, M. Stark, S. Oates, J. Bommer, B. Smith, and H. Asanuma, Induced seismicity associated with Enhanced Geothermal Systems, Geothermics, 36 (3), 185-222, doi:10.1016/j.geothermics.2007.03.003, 2007.

Martínez-Garzón, P., M. Bohnhoff, G. Kwiatek, and G. Dresen, Stress tensor changes related to fluid injection at The Geysers geothermal field California, Geophysical Research Letters, 40(11), 2596-2601, doi:10.1002/grl.50438, 2013. 
Martínez-Garzón, P., G. Kwiatek, M. Ickrath, and M. Bohnhoff, Msatsi: A matlab package for stress inversion combining solid classic methodology, a new simplified user-handling, and a visualization tool, Seismological Research Letters, 85(4), 896904, 2014.

Martínez-Garzón, P., G. Kwiatek, H. Sone, M. Bohnhoff, G. Dresen, and C. Hartline, Spatiotemporal changes faulting regimes, and source parameters of induced seismicity: A case study from The Geysers geothermal field, Journal of Geophysical Research: Solid Earth, 119(11), 8378-8396, doi:10.1002/2014jb011385, 2014.

Martínez-Garzón, P., G. Kwiatek, M. Bohnhoff, and G. Dresen, Volumetric components in the earthquake source related to fluid injection and stress state, Geophysical Research Letters, 44(2), 800-809, doi:10.1002/2016gl071963, 2017.

Massiot, C., and D. D. McNamara, Structural interpretation of acoustic borehole images from well RK18L2, Rotokawa Geothermal Field, GNS Science consultancy report 2010, 216, 43, 2010.

Massiot, C., and D. D. McNamara, Structural interpretation of acoustic borehole images from well RK32, Rotokawa Geothermal Field, GNS Science consultancy report 2011, 64, 42, 2011.

Massiot, C., B. Lewis, and D. D. McNamara, Interpretation of acoustic borehole images from injection well NM8A, Ngatamariki Geothermal Field, GNS Science consultancy report 2012, 192, 24, 2012.

Massiot, C., D. McNamara, and B. Lewis, Processing and analysis of high temperature geothermal acoustic borehole image logs in the taupo volcanic zone, new zealand, Geothermics, 53, 190-201, 2015a.

Massiot, C., J. Townend, D. D. McNamara, and A. Nicol, Fracture width and spacing distributions from borehole televiewer logs and cores in the Rotokawa geothermal field, New Zealand, in World Geothermal Congress 2015, International Geothermal Association, 2015b.

Massiot, C., A. Nicol, D. D. McNamara, and J. Townend, Evidence for tectonic lithologic, and thermal controls on fracture system geometries in an andesitic hightemperature geothermal field, Journal of Geophysical Research: Solid Earth, 122(8), 6853-6874, doi:10.1002/2017jb014121, 2017.

Matson, G., Microseismicity during geothermal stimulation at the Ngatamariki geothermal field: New detections via a matched-filter method, Master's thesis, Victoria University of Wellington, 2019. 
McGarr, A., Maximum magnitude earthquakes induced by fluid injection, Journal of Geophysical Research: solid earth, 119(2), 1008-1019, 2014.

McGarr, A., and A. J. Barbour, Wastewater Disposal and the Earthquake Sequences During 2016 Near Fairview Pawnee, and Cushing, Oklahoma, Geophysical Research Letters, 44(18), 9330-9336, doi:10.1002/2017gl075258, 2017.

McKenzie, D. P., The relation between fault plane solutions for earthquakes and the directions of the principal stresses, Bulletin of the Seismological Society of America, 59(2), 591-601, 1969.

McNamara, D. D., Structural interpretation of acoustic borehole images from well RK30L1, Rotokawa Geothermal Field, GNS Science consultancy report 2010, 124, $34,2010$.

McNamara, D. D., Structural interpretation of acoustic borehole images from well RK29, Rotokawa Geothermal Field, GNS Science consultancy report 2011, 202, 34, 2011.

McNamara, D. D., C. Massiot, B. Lewis, and I. C. Wallis, Heterogeneity of structure and stress in the Rotokawa Geothermal Field New Zealand, Journal of Geophysical Research: Solid Earth, 120(2), 1243-1262, doi:10.1002/2014jb011480, 2015.

McNamara, D. D., S. Sewell, E. Buscarlet, and I. C. Wallis, A review of the Rotokawa Geothermal Field New Zealand, Geothermics, 59, 281-293, doi:10.1016/j. geothermics.2015.07.007, 2016.

Michael, A. J., Determination of stress from slip data: faults and folds, Journal of Geophysical Research: Solid Earth, 89 (B13), 11,517-11,526, 1984.

Michael, A. J., Stress rotation during the coalinga aftershock sequence, Journal of Geophysical Research: Solid Earth, 92(B8), 7963-7979, 1987.

Michael, A. J., Spatial variations in stress within the 1987 whittier narrows, california, aftershock sequence: New techniques and results, Journal of Geophysical Research: Solid Earth, 96(B4), 6303-6319, 1991.

Michelini, A., and A. Lomax, The effect of velocity structure errors on double-difference earthquake location, Geophysical Research Letters, 31(9), 2004.

Minissale, A., The Larderello geothermal field: A review, Earth-Science Reviews, 31 (2), 133-151, doi:10.1016/0012-8252(91)90018-b, 1991.

Mock, J. E., J. W. Tester, and P. M. Wright, Geothermal energy from the earth: its potential impact as an environmentally sustainable resource, Annual review of Energy and the Environment, 22(1), 305-356, 1997. 
Mroczek, S., Seismic anisotropy at the Rotokawa and Ngatamariki geothermal fields in the Taupō Volcanic Zone, Master's thesis, Victoria University of Wellington, 2017.

Mroczek, S., M. Savage, C. Hopp, and S. Sewell, Anisotropy as an indicator of reservoir changes: Example from the Rotokawa and Ngatamariki geothermal fields, New Zealand, Geophysical Journal International, in review, 2019.

Mukuhira, Y., C. Dinske, H. Asanuma, T. Ito, and M. O. Häring, Pore pressure behavior at the shut-in phase and causality of large induced seismicity at Basel Switzerland, Journal of Geophysical Research: Solid Earth, 122(1), 411-435, doi: 10.1002/2016jb013338, 2017.

National Academy of Sciences, Induced Seismicity Potential in Energy Technologies, National Academies Press, doi:10.17226/13355, 2013.

Okada, Y., Internal deformation due to shear and tensile faults in a half-space, Bulletin of the Seismological Society of America, 82(2), 1018-1040, 1992.

Olasolo, P., M. Juárez, M. Morales, S. D’Amico, and I. Liarte, Enhanced geothermal systems (EGS): A review, Renewable and Sustainable Energy Reviews, 56, 133-144, doi:10.1016/j.rser.2015.11.031, 2016.

Oppenheimer, D. H., Extensional tectonics at The Geysers geothermal area, California, Journal of Geophysical Research: Solid Earth, 91 (B11), 11,463-11,476, 1986.

Ormat Technologies Inc., Ormat Technologies Inc., 2017 Annual Report, Tech. rep., 2018.

Pan, S.-Y., M. Gao, K. J. Shah, J. Zheng, S.-L. Pei, and P.-C. Chiang, Establishment of enhanced geothermal energy utilization plans: Barriers and strategies, Renewable Energy, 132, 19-32, doi:10.1016/j.renene.2018.07.126, 2019.

Parotidis, M., S. A. Shapiro, and E. Rothert, Back front of seismicity induced after termination of borehole fluid injection, Geophysical Research Letters, 31(2), doi: 10.1029/2003gl018987, 2004.

Pujol, J., Comments on the joint determination of hypocenters and station corrections, Bulletin of the Seismological Society of America, 78(3), 1179-1189, 1988.

Quinao, J., L. Sirad-Azwar, J. Clearwater, V. Hoepfinger, M. Le Brun, and C. Bardsley, Analyses and modeling of reservoir pressure changes to interpret the Rotokawa geothermal field response to Nga Awa Purua power station operation, in 38th Workshop on Geothermal Reservoir Engineering, 2013. 
Quinao, J. J., E. Buscarlet, and F. Siega, Early identification and management of calcite deposition in the Ngatamariki geothermal field, New Zealand, in Proceedings 42nd Workshop on Geothermal Reservoir Engineering, 2017.

Quinao, J. J. D., and S. J. Zarrouk, Geothermal resource assessment using Experimental Design and Response Surface Methods: The Ngatamariki geothermal field New Zealand, Renewable Energy, 116, 324-334, doi:10.1016/j.renene.2017.09.084, 2018.

Raleigh, C. B., J. H. Healy, and J. D. Bredehoeft, An Experiment in Earthquake Control at Rangely Colorado, Science, 191 (4233), 1230-1237, doi:10.1126/science. 191.4233.1230, 1976.

Rawlinson, Z., Microseismicity associated with actively exploited geothermal systems: Earthquake detection and probabilistic location at Rotokawa and statistical seismic network design at Kawerau, Master's thesis, Victoria University of Wellington, 2011.

Reasenberg, P., and D. Oppenheimer, FPFIT FPPLOT and FPPAGE Fortran computer programs for calculating and displaying earthquake fault-plane solutions, doi: 10.3133/ofr85739, 1985.

Richter, C. F., An instrumental earthquake magnitude scale, Bulletin of the Seismological Society of America, 25(1), 1-32, 1935.

Riffault, J., D. Dempsey, S. Karra, and R. Archer, Microseismicity cloud can be substantially larger than the associated stimulated fracture volume: The case of the Paralana enhanced geothermal system, Journal of Geophysical Research: Solid Earth, doi:10.1029/2017jb015299, 2018.

Risk, G., Electrical resistivity surveys of the Rotokawa geothermal field, New Zealand, in Proceedings of the 22nd New Zealand Geothermal Workshop, pp. 121-126, University of Auckland Auckland, 2000.

Ristau, J., D. Harte, and J. Salichon, A revised local magnitude (ml) scale for new zealand earthquakes, Bulletin of the Seismological Society of America, 106(2), 398407, 2016.

Rowe, C., R. Aster, W. S. Phillips, R. Jones, B. Borchers, and M. Fehler, Using automated, high-precision repicking to improve delineation of microseismic structures at the soultz geothermal reservoir, in The Mechanism of Induced Seismicity, pp. 563-596, Springer, 2002.

Rowland, J. V., and R. H. Sibson, Structural controls on hydrothermal flow in a segmented rift system Taupo Volcanic Zone, New Zealand, Geofluids, 4(4), 259-283, doi:10.1111/j.1468-8123.2004.00091.x, 2004. 
Rubinstein, J. L., and W. L. Ellsworth, Precise Estimation of Repeating Earthquake Moment: Example from Parkfield California, Bulletin of the Seismological Society of America, 100(5A), 1952-1961, doi:10.1785/0120100007, 2010.

Rutledge, J. T., and W. S. Phillips, Hydraulic stimulation of natural fractures as revealed by induced microearthquakes Carthage Cotton Valley gas field, east Texas, GEOPHYSICS, 68(2), 441-452, doi:10.1190/1.1567214, 2003.

Sasaki, S., and H. Kaieda, Determination of stress state from focal mechanisms of microseismic events induced during hydraulic injection at the Hijiori hot dry rock site, in The Mechanism of Induced Seismicity, pp. 489-516, Springer, 2002.

Schaff, D. P., and P. G. Richards, On finding and using repeating seismic events in and near China, Journal of Geophysical Research, 116(B3), doi:10.1029/2010jb007895, 2011.

Schoenball, M., C. Baujard, T. Kohl, and L. Dorbath, The role of triggering by static stress transfer during geothermal reservoir stimulation, Journal of Geophysical Research: Solid Earth, 117(B9), doi:10.1029/2012jb009304, 2012.

Scholz, C. H., The brittle-plastic transition and the depth of seismic faulting, Geologische Rundschau, 777(1), 319-328, doi:10.1007/bf01848693, 1988.

Scholz, C. H., On the stress dependence of the earthquake b value, Geophysical Research Letters, 42(5), 1399-1402, 2015.

Schorlemmer, D., S. Wiemer, and M. Wyss, Variations in earthquake-size distribution across different stress regimes, Nature, 437(7058), 539-542, doi:10.1038/ nature04094, 2005.

Segall, P., Earthquakes triggered by fluid extraction, Geology, 17(10), 942, doi:10.1130/ 0091-7613(1989)017<0942:etbfe $\rangle 2.3 . c o ; 2,1989$.

Segall, P., and S. D. Fitzgerald, A note on induced stress changes in hydrocarbon and geothermal reservoirs, Tectonophysics, 289(1-3), 117-128, doi:10.1016/ s0040-1951(97)00311-9, 1998.

Segall, P., J.-R. Grasso, and A. Mossop, Poroelastic stressing and induced seismicity near the Lacq gas field southwestern France, Journal of Geophysical Research, 99(B8), 15,423, doi:10.1029/94jb00989, 1994.

Sewell, S., S. Addison, L. Azwar, and M. Barnes, Rotokawa Conceptual Model Update 5 years After Commissioning of the 138 MWe NAP Plant, in Proceedings 37th New Zealand Geothermal Workshop, vol. 18, 2015a. 
Sewell, S., M. Savage, J. Townend, S. Bannister, and L. Hutchings, Preliminary investigation of seismic velocity variation at the Rotokawa and Ngatamariki geothermal field, in Proceedings 39th New Zealand Geothermal Workshop, 2017.

Sewell, S. M., W. Cumming, C. J. Bardsley, J. Winick, I. C. Wallis, S. Sherburn, S. Bourguignon, and S. Bannister, Interpretation of Microseismicity at the Rotokawa Geothermal Field, 2008 to 2012, in World Geothermal Congress, 2015b.

Shapiro, S., and C. Dinske, Fluid-induced seismicity: Pressure diffusion and hydraulic fracturing, Geophysical Prospecting, 57(2), 301-310, doi:10.1111/j.1365-2478.2008. 00770.x, 2009.

Shapiro, S. A., E. Huenges, and G. Borm, Estimating the crust permeability from fluid-injection-induced seismic emission at the KTB site, Geophysical Journal International, 131 (2), F15-F18, doi:10.1111/j.1365-246x.1997.tb01215.x, 1997.

Shapiro, S. A., E. Rothert, V. Rath, and J. Rindschwentner, Characterization of fluid transport properties of reservoirs using induced microseismicity, GEOPHYSICS, 67(1), 212-220, doi:10.1190/1.1451597, 2002.

Shapiro, S. A., J. Kummerow, C. Dinske, G. Asch, E. Rothert, J. Erzinger, H.-J. Kümpel, and R. Kind, Fluid induced seismicity guided by a continental fault: Injection experiment of 2004/2005 at the German Deep Drilling Site (KTB), Geophysical Research Letters, 33(1), n/a-n/a, doi:10.1029/2005gl024659, 2006.

Shapiro, S. A., O. S. Krüger, C. Dinske, and C. Langenbruch, Magnitudes of induced earthquakes and geometric scales of fluid-stimulated rock volumes, GEOPHYSICS, 76 (6), WC55-WC63, doi:10.1190/geo2010-0349.1, 2011.

Shelly, D. R., and J. L. Hardebeck, Illuminating faulting complexity of the 2017 yellowstone maple creek earthquake swarm, Geophysical Research Letters, 2018.

Shelly, D. R., G. C. Beroza, and S. Ide, Non-volcanic tremor and low-frequency earthquake swarms, Nature, 446(7133), 305-307, doi:10.1038/nature05666, 2007.

Shelly, D. R., W. L. Ellsworth, and D. P. Hill, Fluid-faulting evolution in high definition: Connecting fault structure and frequency-magnitude variations during the 2014 Long Valley Caldera California, earthquake swarm, Journal of Geophysical Research: Solid Earth, 121(3), 1776-1795, doi:10.1002/2015jb012719, 2016a.

Shelly, D. R., J. L. Hardebeck, W. L. Ellsworth, and D. P. Hill, A new strategy for earthquake focal mechanisms using waveform-correlation-derived relative polarities and cluster analysis: Application to the 2014 Long Valley Caldera earthquake swarm, Journal of Geophysical Research: Solid Earth, 121(12), 8622-8641, doi:10.1002/ 2016jb013437, 2016b. 
Sherburn, S., S. Bannister, and H. Bibby, Seismic velocity structure of the central Taupo Volcanic Zone New Zealand, from local earthquake tomography, Journal of Volcanology and Geothermal Research, 122(1-2), 69-88, doi:10.1016/s0377-0273(02) 00470-5, 2003.

Sherburn, S., S. Sewell, S. Bourguignon, W. Cumming, S. Bannister, C. Bardsley, J. Winick, J. Quinao, and I. Wallis, Microseismicity at Rotokawa geothermal field New Zealand, 2008-2012, Geothermics, 54, 23-34, doi:10.1016/j.geothermics.2014. 11.001, 2015.

Shi, Y., and B. A. Bolt, The standard error of the magnitude-frequency b value, Bulletin of the Seismological Society of America, 72(5), 1677-1687, 1982.

Sibson, R. H., and J. V. Rowland, Stress fluid pressure and structural permeability in seismogenic crust, North Island, New Zealand, Geophysical Journal International, 154(2), 584-594, doi:10.1046/j.1365-246x.2003.01965.x, 2003.

Siega, C., M. Grant, P. Bixley, and W. Mannington, Quantifying the effect of temperature on well injectivity, in Proceedings 36th New Zealand Geothermal Workshop, 2014 .

Simone, S. D., V. Vilarrasa, J. Carrera, A. Alcolea, and P. Meier, Thermal coupling may control mechanical stability of geothermal reservoirs during cold water injection, Physics and Chemistry of the Earth Parts A/B/C, 64, 117-126, doi:10.1016/j.pce. 2013.01.001, 2013.

Skoumal, R. J., M. R. Brudzinski, and B. S. Currie, Earthquakes Induced by Hydraulic Fracturing in Poland Township Ohio, Bulletin of the Seismological Society of America, 105(1), 189-197, doi:10.1785/0120140168, 2015.

Spada, M., T. Tormann, S. Wiemer, and B. Enescu, Generic dependence of the frequency-size distribution of earthquakes on depth and its relation to the strength profile of the crust, Geophysical research letters, 40(4), 709-714, 2013.

Stankova, J., S. L. Bilek, C. A. Rowe, and R. C. Aster, Characteristics of the october 2005 microearthquake swarm and reactivation of similar event seismic swarms over decadal time periods near socorro, new mexico, Bulletin of the Seismological Society of America, 98(1), 93-105, 2008.

Stein, R. S., The role of stress transfer in earthquake occurrence, Nature, 402(6762), 605, 1999.

Stein, S., and M. Wysession, An Introduction to Seismology, Earthquakes and Earth Structure, Blackwell Publishing, 2003. 
Stephens, G., and B. Voight, Hydraulic fracturing theory for conditions of thermal stress, in International Journal of Rock Mechanics and Mining Sciences 83 Geomechanics Abstracts, 6, pp. 279-284, Elsevier, 1982.

Stern, T., and A. Benson, Wide-angle seismic imaging beneath an andesitic arc: Central North Island New Zealand, Journal of Geophysical Research, 116(B9), doi:10.1029/ 2011jb008337, 2011.

Tarantola, A., and B. Valette, Inverse problems= quest for information, J. geophys, $50(3), 150-170,1982$.

The ObsPy Development Team, ObsPy 1.0.1, doi:10.5281/zenodo.48254, 2016.

Thompson, G., Temperature gradients within and adjacent to the taupo volcanic zone, New Zealand Journal of Geology and Geophysics, 23(3), 407-412, 1980.

Thurber, C. H., Nonlinear earthquake location: Theory and examples, Bulletin of the Seismological Society of America, 75(3), 779-790, 1985.

Toda, S., Toggling of seismicity by the 1997 Kagoshima earthquake couplet: A demonstration of time-dependent stress transfer, Journal of Geophysical Research, 108(B12), doi:10.1029/2003jb002527, 2003.

Townend, J., and M. D. Zoback, How faulting keeps the crust strong, Geology, 28(5), 399-402, 2000.

Townend, J., and M. D. Zoback, Implications of earthquake focal mechanisms for the frictional strength of the san andreas fault system, Geological Society, London, Special Publications, 186(1), 13-21, 2001.

Townend, J., and M. D. Zoback, Stress, strain, and mountain building in central japan, Journal of Geophysical Research: Solid Earth, 111(B3), 2006.

Townend, J., S. Sherburn, R. Arnold, C. Boese, and L. Woods, Three-dimensional variations in present-day tectonic stress along the Australia-Pacific plate boundary in New Zealand, Earth and Planetary Science Letters, 353-354, 47-59, doi:10.1016/ j.epsl.2012.08.003, 2012.

Trugman, D. T., and P. M. Shearer, GrowClust: A Hierarchical Clustering Algorithm for Relative Earthquake Relocation with Application to the Spanish Springs and Sheldon, Nevada, Earthquake Sequences, Seismological Research Letters, 88(2A), 379-391, doi:10.1785/0220160188, 2017.

Turin, G., An introduction to matched filters, IEEE Transactions on Information Theory, 6(3), 311-329, doi:10.1109/tit.1960.1057571, 1960. 
Utsu, T., Representation and analysis of the earthquake size distribution: a historical review and some new approaches, in Seismicity Patterns, their Statistical Significance and Physical Meaning, pp. 509-535, Springer, 1999.

VanDecar, J., and R. Crosson, Determination of teleseismic relative phase arrival times using multi-channel cross-correlation and least squares, Bulletin of the Seismological Society of America, 80(1), 150-169, 1990.

Vavryčuk, V., Iterative joint inversion for stress and fault orientations from focal mechanisms, Geophysical Journal International, 199(1), 69-77, 2014.

Villamor, P., K. R. Berryman, I. A. Nairn, K. Wilson, N. Litchfield, and W. Ries, Associations between volcanic eruptions from Okataina volcanic center and surface rupture of nearby active faults Taupo rift, New Zealand: Insights into the nature of volcano-tectonic interactions, Geological Society of America Bulletin, 123(7-8), 1383-1405, doi:10.1130/b30184.1, 2011.

Waldhauser, F., and W. Ellsworth, A Double-Difference Earthquake Location Algorithm: Method and Application to the Northern Hayward Fault California, Bulletin of the Seismological Society of America, 90(6), 1353-1368, doi:10.1785/0120000006, 2000 .

Wallace, L. M., Subduction zone coupling and tectonic block rotations in the North Island New Zealand, Journal of Geophysical Research, 109(B12), doi:10.1029/ 2004jb003241, 2004.

Wallace, R. E., Geometry of shearing stress and relation to faulting, The Journal of geology, 59(2), 118-130, 1951.

Wallis, I., C. Bardsley, T. Powell, J. Rowland, and J. O'Brien, A structural model for the Rotokawa Geothermal Field, New Zealand, in Proceedings 35th New Zealand Geothermal Workshop, 2013.

Walsh, D., R. Arnold, and J. Townend, A Bayesian approach to determining and parametrizing earthquake focal mechanisms, Geophysical Journal International, 176 (1), 235-255, doi:10.1111/j.1365-246x.2008.03979.x, 2009.

Warren, N. W., and G. V. Latham, An experimental study of thermally induced microfracturing and its relation to volcanic seismicity, Journal of Geophysical Research, 75 (23), 4455-4464, doi:10.1029/jb075i023p04455, 1970.

Warren-Smith, E., C. J. Chamberlain, S. Lamb, and J. Townend, High-Precision Analysis of an Aftershock Sequence Using Matched-Filter Detection: The 4 May 2015ML 6 Wanaka Earthquake Southern Alps, New Zealand, Seismological Research Letters, 88(4), 1065-1077, doi:10.1785/0220170016, 2017. 
Warren-Smith, E., S. Lamb, and T. A. Stern, Stress field and kinematics for diffuse microseismicity in a zone of continental transpression, south island, new zealand, Journal of Geophysical Research: Solid Earth, 122(4), 2798-2811, 2017.

Watson, A., Well Measurements from Completion Tests to the First Discharge, in Geothermal Engineering, pp. 99-122, Springer New York, doi:10.1007/ 978-1-4614-8569-8_6, 2013.

Weber, B., J. Becker, W. Hanka, A. Heinloo, M. Hoffmann, T. Kraft, D. Pahlke, J. Reinhardt, and H. Thoms, SeisComP3 - Automatic and interactive real time data processing, in Geophysical Research Abstracts, vol. 9, 2007.

Wei, S., J.-P. Avouac, K. W. Hudnut, A. Donnellan, J. W. Parker, R. W. Graves, D. Helmberger, E. Fielding, Z. Liu, F. Cappa, and M. Eneva, The 2012 Brawley swarm triggered by injection-induced aseismic slip, Earth and Planetary Science Letters, 422, 115-125, doi:10.1016/j.epsl.2015.03.054, 2015.

Wiemer, S., Minimum magnitude of completeness in earthquake catalogs: Examples from Alaska the western United States, and Japan, Bulletin of the Seismological Society of America, 90(4), 859-869, doi:10.1785/0119990114, 2000.

Wiemer, S., and S. R. McNutt, Variations in the frequency-magnitude distribution with depth in two volcanic areas: Mount St. Helens Washington, and Mt. Spurr, Alaska, Geophysical Research Letters, 24(2), 189-192, doi:10.1029/96gl03779, 1997.

Wiemer, S., and M. Wyss, Mapping the frequency-magnitude distribution in asperities: An improved technique to calculate recurrence times?, Journal of Geophysical Research: Solid Earth, 102(B7), 15,115-15,128, 1997.

Wiemer, S., S. R. McNutt, and M. Wyss, Temporal and three-dimensional spatial analyses of the frequency-magnitude distribution near Long Valley Caldera California, Geophysical Journal International, 134(2), 409-421, doi:10.1046/j.1365-246x.1998. 00561.x, 1998.

Wilson, C., B. Houghton, M. McWilliams, M. Lanphere, S. Weaver, and R. Briggs, Volcanic and structural evolution of Taupo Volcanic Zone New Zealand: A review, Journal of Volcanology and Geothermal Research, 68(1-3), 1-28, doi:10.1016/ 0377-0273(95)00006-g, 1995.

Wilson, C., D. Gravley, G. Leonard, and J. Rowland, Volcanism in the central taupo volcanic zone, new zealand: tempo, styles and controls, Studies in volcanology: the legacy of George Walker. Special Publications of IAVCEI, 2, 225-247, 2009. 
Wilson, C. J., and J. V. Rowland, The volcanic magmatic and tectonic setting of the Taupo Volcanic Zone, New Zealand, reviewed from a geothermal perspective, Geothermics, 59, 168-187, doi:10.1016/j.geothermics.2015.06.013, 2016.

Winick, J., T. Powell, and E. Mroczek, The natural-state geochemistry of the Rotokawa reservoir, in Proceedings 31st New Zealand Geothermal Workshop, 2009.

Winick, J., T. Powell, and E. Mroczek, The natural-state geochemistry of the Rotokawa reservoir, in New Zealand Geothermal Workshop 2011 Proceedings, 2011.

Wiprut, D., and M. D. Zoback, Fault reactivation and fluid flow along a previously dormant normal fault in the northern North Sea, Geology, 28(7), 595-598, doi: 10.1130/0091-7613(2000)028<0595:fraffa $\rangle 2.3 . c 0 ; 2,2000$.

Withers, M., R. Aster, C. Young, J. Beiriger, M. Harris, S. Moore, and J. Trujillo, A comparison of select trigger algorithms for automated global seismic phase and event detection, Bulletin of the Seismological Society of America, 88(1), 95-106, 1998.

Yeck, W. L., G. P. Hayes, D. E. McNamara, J. L. Rubinstein, W. D. Barnhart, P. S. Earle, and H. M. Benz, Oklahoma experiences largest earthquake during ongoing regional wastewater injection hazard mitigation efforts, Geophysical Research Letters, 44(2), 711-717, doi:10.1002/2016gl071685, 2017.

Yoon, J. S., A. Zang, and O. Stephansson, Numerical investigation on optimized stimulation of intact and naturally fractured deep geothermal reservoirs using hydromechanical coupled discrete particles joints model, Geothermics, 52, 165-184, doi: 10.1016/j.geothermics.2014.01.009, 2014.

Zhang, H., and C. H. Thurber, Double-difference tomography: The method and its application to the hayward fault, california, Bulletin of the Seismological Society of America, 93(5), 1875-1889, 2003.

Zoback, M. D., Reservoir Geomechanics, Cambridge University Press, 2010.

Zoback, M. D., and S. M. Gorelick, Earthquake triggering and large-scale geologic storage of carbon dioxide, Proceedings of the National Academy of Sciences, 109 (26), 10,164-10,168, doi:10.1073/pnas.1202473109, 2012.

Zoback, M. D., and H.-P. Harjes, Injection-induced earthquakes and crustal stress at 9 km depth at the KTB deep drilling site Germany, Journal of Geophysical Research: Solid Earth, 102(B8), 18,477-18,491, doi:10.1029/96jb02814, 1997.

Zyvoloski, G., Fehm: A control volume finite element code for simulating subsurface multi-phase multi-fluid heat and mass transfer, Los Alamos Unclassified Report LAUR-07-3359, 2007. 